\begin{tabular}{|c|l|}
\hline Title & CONTACT GEOMETRY OF SECOND ORDER II \\
\hline Author(s) & YAMA GUCHI, KEIZO \\
\hline Citation & Hokkaido University Preprint Series in Mathematics, 1017, 1-65 \\
\hline Issue Date & 2012-9-25 \\
\hline DOI & 10.14943/84163 \\
\hline Doc URL & http://hdl.handle.net/2115/69822 \\
\hline Type & bulletin (article) \\
\hline File Information & pre1017.pdf \\
\hline
\end{tabular}

Instructions for use 


\title{
CONTACT GEOMETRY OF SECOND ORDER II
}

\author{
KEIZO YAMAGUCHI
}

\begin{abstract}
This is the continuation of our previous paper "Contact Geometry of Second Order I" , where we have formulated the contact equivalence of systems of second order partial differential equations for a scalar function as the geometry of $P D$ manifolds of second order. In this paper, we will discuss the Two Step Reduction procedure in Contact Geometry of Second Order. In fact we establish the Second Reduction Theorem for $P D$ manifolds $\left(R ; D^{1}, D^{2}\right)$ of second order admitting the first order covariant systems $\tilde{N}$. Utilizing the covariant system $\tilde{N}$, we construct the intermediate object $\left(W ; C^{:}, N\right)$, called the $I G$ manifold of corank $r$, as a submanifold of the Involutive Grassmann bundle $I^{r}(J)$ over the contact manifold $(J, C)$, where $J=R / \mathrm{Ch}\left(D^{1}\right)$. We will seek the condition when the equivalence of $\left(R ; D^{1}, D^{2}\right)$ is reducible to that of $\left(W ; C^{*}, N\right)$. Moreover, when $\mathrm{Ch}(N)$ is non-trivial, the equivalence of $\left(W ; C^{*}, N\right)$ is further reducible to that of $\left(Y ; D_{N}^{*}, D_{N}\right)$, where $Y=W / \mathrm{Ch}(N)$. This theorem gives a sufficient condition for the existence of higher dimensional characteristics of $\left(R ; D^{1}, D^{2}\right)$. By analyzing the construction parts of the Two Step Reduction procedure, we will show several examples of Parabolic Geometries, which are, through the Second Reduction Theorem, associated with the geometry of $P D$ manifolds of second order.
\end{abstract}

\section{INTRODUCTION}

This manuscript is the sequel to Contact Geometry of Second Order I [27], and will discuss the Second Reduction Theorem in Contact Geometry of Second Order. In [27], we have formulated the contact equivalence problem of systems of second order partial differential equations for a scalar function as the geometry of $P D$ (partial differential) manifolds $\left(R ; D^{1}, D^{2}\right)$ of second order, where $R$ is a manifold equipped with a pair of differential systems $D^{1}$ and $D^{2}$ satisfying the appropriate conditions (see $\S 4[27]$ ). We have also established the First Reduction Theorem for $P D$ manifolds $\left(R ; D^{1}, D^{2}\right)$ admitting non-trivial Cauchy characteristic systems $\mathrm{Ch}\left(D^{2}\right)$, which reduces the equivalence of $\left(R ; D^{1}, D^{2}\right)$ to the geometry of $(X, D)$, where $X=R / \mathrm{Ch}\left(D^{2}\right)$ is the leaf space of the foliation $\mathrm{Ch}\left(D^{2}\right)$ on $R$ and $D$ is the differential system on $X$ such that $D^{2}=\rho_{*}^{-1}(D)$ and $\rho: R \rightarrow X$ is the projection. Moreover we have exhibitted several examples of Parabolic Geometries which are, directly or through the First Reduction Theorem, associated with the geometry of $P D$ manifolds of second order.

In this manuscript, we will establish the Second Reduction Theorem for $P D$ manifolds $\left(R ; D^{1}, D^{2}\right)$ admitting the first order covariant systems $\tilde{N}$ such that $D^{1} \supset \tilde{N} \supset D^{2}$. Here $\tilde{N}$ is called covariant if each isomorphism $\varphi$ of $\left(R ; D^{1}, D^{2}\right)$ preserves $\tilde{N}$, i.e., $\varphi_{*}(\tilde{N})=\tilde{N}$. At each point $v \in R$, for every integral element $V$ of $\left(R ; D^{1}, D^{2}\right)$ at $v$, i.e., $V$ is an integral element of $\left(R, D^{2}\right)$ such that $\mathfrak{s}_{-1}(v)=D^{2}(v)=V \oplus \mathrm{Ch}\left(D^{1}\right)(v), \tilde{N}(v)$ defines the $r$-dimensional subspace $E$ of $V$ through the symbol algebra identification; $\mathfrak{s}_{-3}(v)=T_{v}(R) / D^{1}(v) \cong \mathbb{R}, \mathfrak{s}_{-2}(v)=$ $D^{1}(v) / D^{2}(v) \cong V^{*}, \mathrm{Ch}\left(D^{2}\right)(v) \cong \mathfrak{f} \subset S^{2}\left(V^{*}\right)$ so that $\tilde{N}(v) / D^{2}(v) \subset \mathfrak{s}_{-2}(v)$ corresponds to $E^{\perp} \subset V^{*}$, where $\mathfrak{s}(v)=\mathfrak{s}_{-3}(v) \oplus \mathfrak{s}_{-2}(v) \oplus \mathfrak{s}_{-1}(v)$ is the symbol algebra of $\left(R ; D^{1}, D^{2}\right)$ at $v$ and $r$ is the codimension of $\tilde{N}$ in $D^{1}$ (see $\S 3.1$ and $\S 1[27]$ ). Moreover the first order covariant system

1991 Mathematics Subject Classification. 53C15; 58A15; 58A20; 58A30.

Key words and phrases. Contact transformations; Involutive systems of second order; PD manifolds; Reduction Theorems; Parabolic Geomeries. 
$\tilde{N}$ defines the map $\eta: R \rightarrow I^{r}(J)$ by

$$
\eta(v)=p_{*}(\tilde{N}(v)) \in I^{r}(J) \quad \text { for } \quad v \in R,
$$

where $p: R \rightarrow J=R / \mathrm{Ch}\left(D^{1}\right)$ is the projection onto the contact manifold $(J, C)$ of dimension $2 n+1$ such that $D^{1}=p_{*}^{-1}(C)$. Here $I^{r}(J)$ is the Involutive Grassmann Bundle over $(J, C)$ consisting of involutive (coisotropic) subspaces $w$ of codimension $r$ in the symplectic vector space $(C(u), d \varpi)$, i.e., $w^{\perp} \subset w$, where $\varpi$ is a local contact form on $J$ around $u \in J$ and $w^{\perp}=\{X \in C(u) \mid d \varpi(X, Y)=0$ for $\forall Y \in w\}$ (see $\S 2.1$ ). We will consider the image $W=\operatorname{Im}(\eta)$ of this map $\eta: R \rightarrow I^{r}(J)$. Under a mild regularity condition, $W$ is a submanifold of $I^{r}(J)$ such that $W$ carries two differential systems $C^{*}$ and $N$, where $C^{*}$ is the lift of $C$ and $N$ is the restriction to $W$ of the canonical differential system $\bar{N}$ on $I^{r}(J)$ defined by the grassmannian construction (see $\S 2.2) .\left(W ; C^{*}, N\right)$ is called the $I G$ (involutive grassmann) manifold of corank $r$ associated with $\left(R ; D^{1}, D^{2}, \tilde{N}\right)$ (see $\S 3.2$ ). A submanifold $W$ of $I^{r}(J)$ defines a subvariety $\bar{R}(W)$ of the Lagrange Grassmann bundle $L(J)$ over $(J, C)$ by

$$
\bar{R}(W)=\{v \in L(J) \mid v \subset \exists w \in W\} .
$$

Technically we construct the Lagrange Grassmann bundle $R(W)$ over $\left(W ; C^{*}, N\right)$ by

$$
R(W)=\bigcup_{w \in W} R_{w}, \quad R_{w}=\left\{\hat{v} \subset N(w)|d \hat{\omega}|_{\hat{v}}=0, \quad \hat{v} \text { is maximal }\right\},
$$

where $C^{*}=\{\hat{\varpi}=0\}$ around $w \in W$. Then we have a map $\zeta: R(W) \rightarrow L(J)$ defined by $\zeta(\hat{v})=$ $q_{*}(\hat{v})$, where $q: W \rightarrow J$ is the projection and $\bar{R}(W)=\zeta(R(W))$. Here $\zeta\left(R_{w}\right) \cong L\left(w / w^{\perp}\right)$ is the Lagrange Grassmann manifold of the symplectic vector space $w / w^{\perp}$ of dimension $2(n-r)$ (see $\S 4.1$ ). Moreover $R(W)$ carries three differential systems $D_{W}^{1}, D_{W}^{2}$ and $N_{W}$, where $D_{W}^{1}$ and $N_{W}$ are lifts of $C^{*}$ and $N$ respectively and $D_{W}^{2}$ is the canonical system defined by the grassmmannian construction (see $\S 4.2$ ). Furthermore we have a map $\kappa_{1}: R \rightarrow R(W)$ defined by

$$
\kappa_{1}(v)=\eta_{*}\left(D^{2}(v)\right) \in R_{w}, \quad w=\eta(v) .
$$

$\kappa_{1}$ is actually an immersion by the Realization Lemma and preserves $D^{1}, D^{2}$ and $\tilde{N}$, i.e., $\left(\kappa_{1}\right)_{*}^{-1}\left(D_{W}^{1}\right)=D^{1},\left(\kappa_{1}\right)_{*}^{-1}\left(D_{W}^{2}\right)=D^{2}$ and $\left(\kappa_{1}\right)_{*}^{-1}\left(N_{W}\right)=\tilde{N}$ (see $\left.\S 5.1\right)$. We will seek the condition for $\kappa_{1}:\left(R ; D^{1}, D^{2}\right) \rightarrow\left(R(W) ; D_{W}^{1}, D_{W}^{2}\right)$ to be a local isomorphism, which gives us the first part of the Second Reduction Theorem. For this purpose, we consider the following covariant systems $\mathfrak{f}(E)$ and $C(E)$ of $\left(R ; D^{1}, D^{2}\right)$ : As a regularity condition for $\left(R ; D^{1}, D^{2}\right)$, we assume the constancy of symbol algebras, i.e., the symbol algebra $\mathfrak{s}(v)$ of $\left(R ; D^{1}, D^{2}\right)$ at each $v \in R$ is isomorphic to a fixed subalgebra $\mathfrak{s}$ of $\mathfrak{c}^{2}(n)$, where $\mathfrak{s}=\mathfrak{s}_{-3} \oplus \mathfrak{s}_{-2} \oplus \mathfrak{s}_{-1}$ is defined by

$$
\mathfrak{s}_{-3}=\mathbb{R}, \quad \mathfrak{s}_{-2}=V^{*}, \quad \mathfrak{s}_{-1}=V \oplus \mathfrak{f}, \quad \mathfrak{f} \subset S^{2}\left(V^{*}\right) .
$$

Under this regurality condition, $\left(R ; D^{1}, D^{2}\right)$ is called regular of type $\mathfrak{s}$. In this case, the first covariant system $\tilde{N}$ of $\left(R ; D^{1}, D^{2}\right)$ corresponds to the $G_{0}(\mathfrak{s})$-invariant subspace $E \subset V$, where $G_{0}(\mathfrak{s})=\{\sigma \in G(\mathfrak{s}) \mid \sigma(V)=V\}$ and $G(\mathfrak{s})$ is the group of graded Lie algebra automorphisms of $\mathfrak{s}$ (see $\S 3.1$ ). We define subspaces $\mathfrak{f}_{E}$ and $\mathfrak{c}_{E}$ of $\mathfrak{f}$ and $\mathfrak{s}_{-1}$ by

$$
\mathfrak{f}_{E}=\mathfrak{f} \cap S^{2}\left(E^{\perp}\right) \subset \mathfrak{f}, \quad \mathfrak{c}_{E}=\hat{E} \oplus \mathfrak{f}_{E} \subset \mathfrak{s}_{-1}=V \oplus \mathfrak{f},
$$

where $\hat{E}=\left\{v \in E \mid v \odot E \subset \mathfrak{f}^{\perp}\right\}$ and $\mathfrak{f}^{\perp}$ is the annihilator of $\mathfrak{f}$ in $S^{2}(V) \cong\left(S^{2}\left(V^{*}\right)\right)^{*}$. Then, by the $G_{0}(\mathfrak{s})$-invariance of $E$, we can define covariant systems $\mathfrak{f}(E)$ and $C(E)$ by

$$
\mathfrak{f}(E)(v)=\phi^{-1}\left(\mathfrak{f}_{E}\right) \subset C(E)(v)=\phi^{-1}\left(\mathfrak{c}_{E}\right) \subset D^{2}(v)=\mathfrak{s}_{-1}(v) \quad \text { for } \quad v \in R,
$$

where $\phi$ is a graded Lie algebra isomorphism of $\mathfrak{s}(v)$ onto $\mathfrak{s}$. By a symbol algebra calculation, we have $\operatorname{Ch}(\tilde{N}) \subset C(E)$ (see $\S 5.2$ ). In fact we will obtain the first part of Second Reduction 
Theorem for $P D$ manifolds of second order admitting the first order covariant systems as follows.

Theorem 5.1. Let $\left(R ; D^{1}, D^{2}\right)$ be a PD manifold of second order, which is regular of type $\mathfrak{s}$. Assume that there exists $G_{0}(\mathfrak{s})$-invariant subspace $E$ of $V$ of dimension $r$ such that $\mathfrak{f}^{\perp} \subset E \otimes_{S} V$.

(1) In case $r<n-1$, if $\mathfrak{f}(E)$ is completely integrable, then $\mathfrak{f}(E)=C h\left(D^{1}\right) \cap C h(\tilde{N})$ and $\kappa_{1}:\left(R ; D^{1}, D^{2}\right) \rightarrow\left(R(W) ; D_{W}^{1}, D_{W}^{2}\right)$ is a local isomorphism.

(2) In case $r<n-1$, if $C(E)$ is completely integrable, then $C(E)=C h(\tilde{N})$ and $\kappa_{1}$ : $\left(R ; D^{1}, D^{2}\right) \rightarrow\left(R(W) ; D_{W}^{1}, D_{W}^{2}\right)$ is a local isomorphism.

(3) In case $r=n-1$, further assume that rank $C h\left(D^{2}\right)<\operatorname{dim} \hat{E}$, if $C(E)$ is completely integrable, then $C(E)=C h(\tilde{N})$ and $\kappa_{1}:\left(R ; D^{1}, D^{2}\right) \rightarrow\left(R(W) ; D_{W}^{1}, D_{W}^{2}\right)$ is a local isomorphism.

When $\kappa_{1}:\left(R ; D^{1}, D^{2}\right) \rightarrow\left(R(W) ; D_{W}^{1}, D_{W}^{2}\right)$ is a local isomorphism, the (local) integration problem of $\left(R ; D^{1}, D^{2}\right)$ is reduced to that of $\left(W ; C^{*}, N\right)$ (see $\left.\S 5.1\right)$. Moreover, for every integral element $V$ of $\left(R ; D^{1}, D^{2}\right)$ at $v \in R, \tilde{N}(v)$ defines the $r$-dimentional subspace $E$ of $V$. Then the condition $\mathfrak{f}^{\perp} \subset E \otimes_{S} V$ implies that $E$ is a Monge element (Definition 7.9 [20], see also Proposition 7.4, Lemmas $7.6[20]$ ). In this case, $C(E)$ does not necessarily coincide with the Monge characteristic system corresponding to $E$ in the sence of $\S 7.3$ [20] (see $\S 6$, Remark 6.2 (1)).

A little generally the submanifold theory of $I^{r}(J)$ will be formulated as the geometry of $I G$ manifolds $\left(W ; C^{*}, N\right)$ of corank $r$ in $\S 2.2$. Moreover we will describe the condition, when $\left(R(W) ; D_{W}^{1}, D_{W}^{2}\right)$ becomes a $P D$ manifold, in terms of covariant systems of $\left(W ; C^{*}, N\right)$ in $\S 4.2$.

Furthermore, in the above Theorem, when $\hat{E} \neq\{0\}$, we have the second step reduction procedure for $\left(W ; C^{*}, N\right)$, similarly as in the case of the First Reduction Theorem for $\left(R ; D^{1}, D^{2}\right)$ admitting non-trivial Cauchy characteristic system $\mathrm{Ch}\left(D^{2}\right)$, as follows (see $\left.\S 5.3\right)$ : Assume that $C(E)=\operatorname{Ch}(\tilde{N})$. When $\hat{E} \neq\{0\}$, since $\tilde{N}=\eta_{*}^{-1}(N), N$ has non-trivial Cauchy characteristic system $\operatorname{Ch}(N)$ on $W$ such that $\operatorname{rank} \operatorname{Ch}(N)=\operatorname{dim} \hat{E}=s>0$. Here assume that $W$ is regular with respect to $\mathrm{Ch}(N)$, i.e., the space $Y=W / \mathrm{Ch}(N)$ of leaves of this foliation is a manifold such that each fibre of the projection $\beta: W \rightarrow Y$ is connected and $\beta$ is a submersion. We further assume that $C(E) \subset \mathrm{Ch}\left(\tilde{N}^{*}\right)$, where $\tilde{N}^{*}$ is the first order covariant system of $\left(R ; D^{1}, D^{2}\right)$ corresponding to $\hat{E}$. Then $\mathrm{Ch}(N) \subset \mathrm{Ch}\left(N^{*}\right) \subset N$ on $W$, where $N^{*}$ is a covariant system of $\left(W ; C^{*}, N\right)$ such that $\eta_{*}^{-1}\left(N^{*}\right)=\tilde{N}^{*}$ (see $\left.\S 2.3\right)$. Hence there exist differential systems $D_{N}^{*}$ and $D_{N}$ on $Y$ of codimension $s+1$ and $r+1$ respectively such that $N^{*}=\beta_{*}^{-1}\left(D_{N}^{*}\right), N=\beta_{*}^{-1}\left(D_{N}\right)$, $D_{N} \supset \mathrm{Ch}\left(D_{N}^{*}\right)$ and $\mathrm{Ch}\left(D_{N}\right)$ is trivial. In this situation, from $\left(Y ; D_{N}^{*}, D_{N}\right)$, we can reconstruct the $I G$ manifold $\left(W ; C^{*}, N\right)$, at least locally, as follows. First let us consider the collection $\tilde{W}(Y)$ of hyperplanes $w$ in each tangent space $T_{y}(Y)$ at $y \in Y$ which contains the fibre $D_{N}^{*}(y)$ of $D_{N}^{*}$.

$$
\tilde{W}(Y)=\bigcup_{y \in Y} \tilde{W}_{y}, \quad \tilde{W}_{y}=\left\{w \in G r\left(T_{y}(Y), m-1\right) \mid w \supset D_{N}^{*}(y)\right\} \cong P\left(T_{y}(Y) / D_{N}^{*}(y)\right)=\mathbb{P}^{s},
$$

where $m=\operatorname{dim} Y$ and $s=\operatorname{dim} \hat{E}$. Moreover $C_{Y}^{*}$ is the canonical system obtained by the grassmannian construction and $N_{Y}^{*}, N_{Y}$ are the lifts of $D_{N}^{*}, D_{N}$. Then we have a map $\kappa_{2}$ of $W$ into $\tilde{W}(Y)$ given by

$$
\kappa_{2}(w)=\beta_{*}\left(C^{*}(w)\right) \subset T_{y}(Y),
$$

for each $w \in W$ and $y=\beta(w)$. In fact $\kappa_{2}:\left(W ; C^{*}, N\right) \rightarrow\left(\tilde{W}(Y) ; C_{Y}^{*}, N_{Y}\right)$ is a local isomorphism (see $\S 5.3)$. Thus $\left(W ; C^{*}, N\right)$ is reconstructed from $\left(Y ; D_{N}^{*}, D_{N}\right)$, at least locally, as a part of $\left(\tilde{W}(Y) ; C_{Y}^{*}, N_{Y}\right)$. 
Now the main theorem of this manuscript (Two Step Reduction Theorem for PD manifolds of second order) can be stated as follows.

Theorem 5.2. Let $\left(R ; D^{1}, D^{2}\right)$ and $\left(\hat{R} ; \hat{D}^{1}, \hat{D}^{2}\right)$ be PD manifolds of second order, which are regular of type $\mathfrak{s}$. Assume that there exists a $G_{0}(\mathfrak{s})$-invariant subspace $E$ of $V$ of dimension $r$ satisfying $\mathfrak{f}^{\perp} \subset E \otimes_{S} V$ and $\operatorname{dim} \hat{E}=s>0$. Moreover assume the following two conditions for the covariant sytems of each PD manifold;

(i) $C(E)$ and $\hat{C}(E)$ are completely integrable (when $r=n-1$, assume further rank $C h\left(D^{2}\right)<$ $s$ and $\left.\operatorname{rank} C h\left(\hat{D}^{2}\right)<s\right)$.

(ii) $C(E) \subset C h\left(\tilde{N}^{*}\right)$ and $\hat{C}(E) \subset C h\left(\hat{N}^{*}\right)$.

Let $\left(W ; C^{*}, N\right)$ and $\left(\hat{W} ; \hat{C}^{*}, \hat{N}\right)$ be the associated $I G$ manifolds of corank $r$ of $\left(R ; D^{1}, D^{2}\right)$ and $\left(\hat{R} ; \hat{D}^{1}, \hat{D}^{2}\right)$ respectively. Assume that $W$ and $\hat{W}$ are regular with respect to $C h(N)$ and $C h(\hat{N})$ respectively. Let $\left(Y ; D_{N}^{*}, D_{N}\right)$ and $\left(\hat{Y}, D_{\hat{N}}^{*}, D_{\hat{N}}\right)$ be the leaf spaces, where $Y=W / C h(N)$ and $\hat{Y}=\hat{W} / C h(\hat{N})$. Let us fix points $v_{o} \in R$ and $\hat{v}_{o} \in \hat{R}$ and put $w_{o}=\eta\left(v_{o}\right), y_{o}=\beta\left(w_{o}\right)$ and $\hat{w}_{o}=\hat{\eta}\left(\hat{v}_{o}\right), \hat{y}_{o}=\hat{\beta}\left(\hat{w}_{o}\right)$. Then a local isomorphism $\psi:\left(R ; D^{1}, D^{2}\right) \rightarrow\left(\hat{R} ; \hat{D}^{1}, \hat{D}^{2}\right)$ such that $\psi\left(v_{o}\right)=\hat{v}_{o}$ induces a local isomorphism $\varphi:\left(W ; C^{*}, N\right) \rightarrow\left(\hat{W}, \hat{C}^{*}, \hat{N}\right)$ such that $\varphi\left(w_{o}\right)=\hat{w}_{o}$ and $\varphi_{*}\left(\kappa_{1}\left(v_{o}\right)\right)=\hat{\kappa}_{1}\left(\hat{v}_{o}\right)$, and vice versa. Furthermore a local isomorphism $\varphi:\left(W ; C^{*}, N\right) \rightarrow$ $\left(\hat{W}, \hat{C}^{*}, \hat{N}\right)$ such that $\varphi\left(w_{o}\right)=\hat{w}_{o}$ induces a local isomorphism $\phi:\left(Y ; D_{N}^{*}, D_{N}\right) \rightarrow\left(\hat{Y} ; D_{\hat{N}}^{*}, D_{\hat{N}}\right)$ such that $\phi\left(y_{o}\right)=\hat{y}_{o}$ and $\phi_{*}\left(\kappa_{2}\left(w_{o}\right)\right)=\hat{\kappa}_{2}\left(\hat{w}_{o}\right)$, and vice versa.

Here we remark that, when $\hat{E}$ coincides with $E$, i.e., when $s=r$, we have $N^{*}=N$ and $D_{N}^{*}=D_{N}$. Hence, in this case, the condition $(i i)$ is automatically satisfied under the condition (i) and the equivalence of $\left(R ; D^{1}, D^{2}\right)$ is reducible to that of $\left(Y, D_{N}\right)$.

In Theorem 5.2, every (local) solution of $\left(W ; C^{*}, N\right)$ is foliated by $\mathrm{Ch}(N)$ so that every (local) solution of $\left(R ; D^{1}, D^{2}\right)$ is foliated by $s$-dimensional integral manifolds of $C(E)=\mathrm{Ch}(\tilde{N})$. Thus $\left(R ; D^{1}, D^{2}\right)$, satisfying the conditions in Theorem 5.2 , admits $s$-dimensional characteristics. In fact, in [6], for involutive systems of second order partial differential equations for a scalar function with 3 independent variables, E.Cartan first classified involutive subspaces $\mathfrak{f} \subset S^{2}\left(V^{*}\right)$ when $\operatorname{dim} V=3$, and immediately wrote the Structure Equation for each involutive system. Then he argued about the existence of 1 or 2-dimensional characteristics for such systems. As for the existemce of characteristics, Theorem 5.2 covers many of his arguments (see $\S 8.1$ ).

In [23], we have exhibitted typical examples of involutive symbols $\mathfrak{f}=\mathfrak{f}^{1}(r), \mathfrak{f}^{2}(r)$ or $\mathfrak{f}^{3}(r)$ in $S^{2}\left(V^{*}\right)$, which are the only invariants of the involutive systems of second order of type $\mathfrak{s}$, where $\mathfrak{s}_{-1}=V \oplus \mathfrak{f}$. Namely we have exhibitted that, for a $P D$ manifold $\left(R ; D^{1}, D^{2}\right)$ of second order, which is regular of type $\mathfrak{s}$, where $\mathfrak{f}$ is $\mathfrak{f}^{1}(r), \mathfrak{f}^{2}(r)$ or $\mathfrak{f}^{3}(r), R$ can be transformed to the model linear equation by a contact transformation, similarly as in the case of the system of first order partial differential equation for a scalar function (see Theorem in [23]). We will explain these phenomena for $\mathfrak{f}^{2}(r)$ and $\mathfrak{f}^{1}(r)$ in terms of the Second Reduction Theorem in $\S 6$ (the explanation for $\mathfrak{f}^{3}(r)$ has been given in terms of the First Reduction Theorem in $\S 6.1$ [27]).

In $\S 7$, we will discuss the construction parts of the Two Step Reduction procedure of Theorem 5.2 , depending on whether $D_{N}^{*}=D_{N}$ or not. Finally in $\S 8$, as examples of Second Reduction Theorem, we first review the arguments in [6] and, in the rest of this section, we will show several examples of Parabolic Geometries, which are, through the Second Reduction Theorem, associated with the geometry of $P D$ manifolds of second order.

Throughout this manuscript we always assume the differentiability of class $C^{\infty}$, though the argument goes through in real or complex analytic category with suitable modifications. 


\section{Table of Contents}

$\S 2$. Involutive Grassmann Bundle over a Contact Manifold $(J, C)$.

2.1. Involutive Grassmann Bundle $I^{r}(J)$.

2.2. $I G$ manifold $(W ; C: N)$ of corank $r$.

2.3. Covariant systems of $\left(W ; C^{*}, N\right)$.

$\S 3 . \quad I G$ manifolds induced from $P D$ manifolds of second order.

3.1. Covariant Systems associated with a $P D$ manifold of second order.

3.2. $I G$ manifold $\left(W ; C^{*}, N\right)$ associated with $\left(R ; D^{1}, D^{2}, \tilde{N}(E)\right)$.

$\S 4$. Lagrange Grassmann Bundle $R(W)$ over an $I G$ manifold $\left(W ; C^{*}, N\right)$.

4.1. Lagrange Grassmann Bundle $R(W)$.

4.2. Differential Systems on $R(W)$.

$\S 5$. Second Reduction Theorem.

5.1. Equivalence of $\left(R ; D^{1}, D^{2}\right)$ and $(W ; C: N)$.

5.2. Covariant systems $\mathfrak{f}(E)$ and $C(E)$.

5.3. Two Step Reduction.

$\S 6$. Typical Classes and their Generalizations.

6.1. Minimum subspace $F$ satisfying $\mathfrak{f}^{\perp} \subset S^{2}(F)$.

6.2. Typical Class of Type $\mathfrak{f}^{2}(r)$ and its Generalization.

6.3. Typical Class of Type $\mathfrak{f}^{1}(r)$ and its Generalization.

$\S 7$. Construction of $\left(W(Y) ; C_{Y}^{*}, N_{Y}\right)$ and $\left(R(Y) ; D_{Y}^{1}, D_{Y}^{2}\right)$.

7.1. Case $N^{*}=N$.

7.2. General Case.

$\S 8 . \quad$ Examples of Second Reduction Theorem.

8.1. Case $n=3$.

8.2. Case $N^{*}=N$.

8.3. General Case.

8.4. Other Examples.

\section{Involutive Grassmann Bundle over a Contact Manifold $(J, C)$}

2.1. Involutive Grassmann Bundle $I^{r}(J)$. Let $(J, C)$ be a contact manifold of dimension $2 n+1$, i.e., $J$ is a manifold of dimension $2 n+1$ and $C$ is a subbundle of the tangent bundle $T(J)$ of $J$ of corank 1 such that the Cauchy characteristic system $\mathrm{Ch}(C)$ of $C$ is trivial. We will consider the Involutive Grassmann Bundle $I^{r}(J)$ of $(J, C)$ of codimension $r(1 \leqq r<n)$;

$$
I^{r}(J)=\bigcup_{u \in J} I_{u} \stackrel{\pi}{\longrightarrow} J, \quad I_{u}=\{\text { involutive subspaces } w \text { of }(C(u), d \varpi) \text { of codimension } r\},
$$

i.e., $w$ is a subspace of the symplectic vector space $(C(u), d \varpi)$ of codimension $r$ such that $w^{\perp} \subset$ $w$, where $C=\{\varpi=0\}$ around $u$ and $w^{\perp}=\{X \in C(u) \mid d \varpi(X, Y)=0$ for $\forall Y \in w\}$. Let us fix a reference point $w_{o}$ of $I^{r}(J)$ and put $u_{o}=\pi\left(w_{o}\right)$, where $\pi: I^{r}(J) \rightarrow J$ is the projection. Take a canonical coordinate system $\left(x_{1}, \ldots, x_{n}, z, p_{1}, \ldots, p_{n}\right)$ of $(J, C)$ defined on a neighborhood $U^{\prime}$ with origin $u_{o}$ such that $w_{o}=\left\{X \in T_{u_{o}}(J) \mid \varpi(X)=d p_{1}(X)=\cdots=d p_{r}(X)=0\right\}$, where $\varpi=d z-\sum_{i=1}^{n} p_{i} d x_{i}$. We can introduce coordinate system $\left(x_{i}, x_{\alpha}, z, p_{i}, p_{\alpha}, a_{i}^{\alpha}, b_{i}^{\alpha}, s_{i j}\right)$ $(1 \leqq i \leqq j \leqq r, r+1 \leqq \alpha \leqq n)$ of $I^{r}(J)$ on the following neighborhood $\bar{U}$ of $w_{o}$;

$$
\bar{U}=\left\{w \in \pi^{-1}\left(U^{\prime}\right) \mid d p_{r+1}, \ldots, d p_{n}, d x_{1}, \ldots, d x_{n} \text { are linearly independent on } w\right\} .
$$


By expressing $\left.d p_{i}\right|_{w}$ as a linear combination of $\left.d p_{\alpha}\right|_{w},\left.d x_{i}\right|_{w}$ and $\left.d x_{\alpha}\right|_{w}$, we see that $w$ is defined by

$$
w=\left\{X \in T_{u}(J) \mid \varpi(X)=\varpi_{1}^{*}(X)=\cdots=\varpi_{r}^{*}(X)=0\right\},
$$

where $u=\pi(w)$ and

$$
\varpi=d z-\sum_{a=1}^{n} p_{a} d x_{a}, \quad \varpi_{i}^{*}=d p_{i}-\sum_{\alpha=r+1}^{n} a_{i}^{\alpha} d p_{\alpha}-\sum_{\alpha=r+1}^{n} b_{i}^{\alpha} d x_{\alpha}-\sum_{j=1}^{r}\left(s_{i j}-\sum_{\alpha=r+1}^{n} a_{i}^{\alpha} b_{j}^{\alpha}\right) d x_{j}
$$

for $1 \leqq i \leqq r$. We calculate

$$
\begin{aligned}
d \varpi= & \sum_{i=1}^{r} d x_{i} \wedge d p_{i}+\sum_{\alpha=r+1}^{n} d x_{\alpha} \wedge d p_{\alpha} \\
= & \sum_{i=1}^{r} d x_{i} \wedge \varpi_{i}^{*}+\sum_{\alpha=r+1}^{n} d x_{\alpha} \wedge d p_{\alpha}+\sum_{i, \alpha} a_{i}^{\alpha} d x_{i} \wedge d p_{\alpha} \\
& \quad+\sum_{i, \alpha} b_{i}^{\alpha} d x_{i} \wedge d x_{\alpha}+\sum_{i, j} s_{i j} d x_{i} \wedge d x_{j}-\sum_{i, j, \alpha} a_{i}^{\alpha} b_{j}^{\alpha} d x_{i} \wedge d x_{j}, \\
= & \sum_{i=1}^{r} d x_{i} \wedge \varpi_{i}^{*}+\sum_{\alpha=r+1}^{n} \omega^{\alpha} \wedge \varpi_{\alpha}^{*}+\sum_{i, j=1}^{r} s_{i j} d x_{i} \wedge d x_{j} \\
\equiv & \sum_{\alpha=r+1}^{n} \omega^{\alpha} \wedge \varpi_{\alpha}^{*}+\sum_{i, j=1}^{r} s_{i j} d x_{i} \wedge d x_{j} \quad\left(\bmod \varpi, \varpi_{1}^{*}, \ldots, \varpi_{r}^{*}\right)
\end{aligned}
$$

where we put

$$
\omega^{\alpha}=d x_{\alpha}+\sum_{i=1}^{r} a_{i}^{\alpha} d x_{i}, \quad \varpi_{\alpha}^{*}=d p_{\alpha}-\sum_{j=1}^{r} b_{j}^{\alpha} d x_{j} \quad(r+1 \leqq \alpha \leqq n) .
$$

Here $w$ is involutive if and only if $\left.d \varpi\right|_{w}$ is of rank $2(n-r)$, which implies $s_{i j}=s_{j i}(1 \leqq i, j \leqq r)$. Moreover we calculate

$$
\begin{aligned}
d \varpi_{i}^{*} & =\sum_{\alpha=r+1}^{n} d p_{\alpha} \wedge d a_{i}^{\alpha}+\sum_{\alpha=r+1}^{n} d x_{\alpha} \wedge d b_{i}^{\alpha}+\sum_{j=1}^{r} d x_{j} \wedge d s_{i j}-\sum_{j, \alpha} d x_{j} \wedge\left(a_{i}^{\alpha} d b_{j}^{\alpha}+b_{j}^{\alpha} d a_{i}^{\alpha}\right) \\
& =\sum_{\alpha=r+1}^{n} \varpi_{\alpha}^{*} \wedge d a_{i}^{\alpha}+\sum_{\alpha=r+1}^{n} \omega^{\alpha} \wedge d b_{i}^{\alpha}+\sum_{j=1}^{r} d x_{j} \wedge \varpi_{i j}
\end{aligned}
$$


where $\varpi_{i j}=d s_{i j}-\sum_{\alpha=r+1}^{n}\left(a_{i}^{\alpha} d b_{j}^{\alpha}+a_{j}^{\alpha} d b_{i}^{\alpha}\right)(1 \leqq i, j \leqq r)$. Hence, for these 1 -forms, we have

$$
\left\{\begin{aligned}
d \varpi & =\sum_{i=1}^{r} d x_{i} \wedge \varpi_{i}^{*}+\sum_{\alpha=r+1}^{n} \omega^{\alpha} \wedge \varpi_{\alpha}^{*} \\
d \varpi_{i}^{*} & =\sum_{\alpha=r+1}^{n} \varpi_{\alpha}^{*} \wedge d a_{i}^{\alpha}+\sum_{\alpha=r+1}^{n} \omega^{\alpha} \wedge d b_{i}^{\alpha}+\sum_{j=1}^{r} d x_{j} \wedge \varpi_{i j} \\
d \varpi_{\alpha}^{*}=\sum_{i=1}^{r} d x_{i} \wedge d b_{i}^{\alpha} & \\
d \omega^{\alpha} & =\sum_{i=1}^{r} d a_{i}^{\alpha} \wedge d x_{i} \\
d \varpi_{i j} & =\sum_{\alpha=r+1}^{n}\left(d b_{i}^{\alpha} \wedge d a_{j}^{\alpha}+d b_{j}^{\alpha} \wedge d a_{i}^{\alpha}\right)
\end{aligned}\right.
$$

We have several differential systems naturally defined on $I^{r}(J)$ as follows: $\bar{C}=\pi_{*}^{-1}(C)$ is the lift of $C$ and canonical systems $\bar{N}, \bar{N}^{\perp}$ are defined by

$$
\bar{N}^{\perp}(w)=\pi_{*}^{-1}\left(w^{\perp}\right) \subset \bar{N}(w)=\pi_{*}^{-1}(w) \subset T_{w}\left(I^{r}(J)\right) \stackrel{\pi_{*}}{\longrightarrow} T_{u}(J) \quad \text { at each } \quad w \in I^{r}(J) .
$$

By the above calculation, we have, on a neighborhood $\bar{U}, \bar{C}=\{\varpi=0\}$ and $\bar{N}=\left\{\varpi=\varpi_{1}^{*}=\cdots=\varpi_{r}^{*}=0\right\}, \quad \bar{N}^{\perp}=\left\{\varpi=\varpi_{i}^{*}=\varpi_{\alpha}^{*}=\omega^{\alpha}=0(1 \leqq i \leqq r, r+1 \leqq \alpha \leqq n)\right\}$.

For the Cauchy characteristic systems of these systems, we see $\operatorname{Ch}(\bar{N})=\operatorname{Ch}\left(\bar{N}^{\perp}\right)=\{0\}$ and

$$
\operatorname{Ch}(\bar{C})=\left\{\varpi=\varpi_{i}^{*}=\varpi_{\alpha}^{*}=d x_{i}=\omega^{\alpha}=0 \quad(1 \leqq i \leqq r, r+1 \leqq \alpha \leqq n)\right\} .
$$

The dual frame of the coframe $\left\{\varpi, \varpi_{i}^{*}, \varpi_{\alpha}^{*}, \omega^{\alpha}, d x_{i}, d b_{i}^{\alpha}, d a_{i}^{\alpha}, \varpi_{i j}(1 \leqq i \leqq j \leqq r, r+1 \leqq \alpha \leqq n)\right\}$ on $\bar{U}$ consists of the following vector fields;

$$
\begin{aligned}
\frac{\partial}{\partial z}, \quad \frac{\partial}{\partial p_{i}}, \quad \frac{d^{*}}{d p_{\alpha}}=\frac{\partial}{\partial p_{\alpha}}+\sum_{j=1}^{r} a_{j}^{\alpha} \frac{\partial}{\partial p_{j}}, \quad \frac{d^{*}}{d x_{\alpha}}=\frac{\partial}{\partial x_{\alpha}}+p_{\alpha} \frac{\partial}{\partial z}+\sum_{j=1}^{r} b_{j}^{\alpha} \frac{\partial}{\partial p_{j}}, \\
\frac{d^{*}}{d x_{i}}=\frac{\partial}{\partial x_{i}}+p_{i} \frac{\partial}{\partial z}+\sum_{j=1}^{r}\left(s_{i j}-\sum_{\alpha=r+1}^{n} a_{j}^{\alpha} b_{i}^{\alpha}\right) \frac{\partial}{\partial p_{j}}+\sum_{\alpha=r+1}^{n} b_{i}^{\alpha} \frac{d^{*}}{d p_{\alpha}}-\sum_{\alpha=r+1}^{n} a_{i}^{\alpha} \frac{d^{*}}{d x_{\alpha}}, \\
\frac{d^{*}}{d b_{i}^{\alpha}}=\frac{\partial}{\partial b_{i}^{\alpha}}+\sum_{j=1}^{r} a_{j}^{\alpha} \frac{\partial}{\partial s_{i j}}+a_{i}^{\alpha} \frac{\partial}{\partial s_{i i}}, \frac{\partial}{\partial a_{i}^{\alpha}}, \quad \frac{\partial}{\partial s_{i j}} .
\end{aligned}
$$

Hence, by calculating brackets of the above vector fields, or by (2.1), we obtain

$$
\partial \bar{N}^{\perp}=\bar{C}, \quad \partial \bar{N}=T\left(I^{r}(J)\right),
$$

where $\partial D$ denotes the derived system of $D$. Moreover, putting

$$
A(\bar{C})=\left\{X \in \operatorname{Ch}(\bar{C}) \mid \varpi_{i j}(X)=0 \quad(1 \leqq i, j \leqq r)\right\},
$$

we have $\partial(A(\bar{C}))=\mathrm{Ch}(\bar{C})$. Here we note that $A(\bar{C})$ corresponds to the standard diffrential system of each fibre (the Involutive Grassmann manifold of codimension $r$ ) of the projection $\pi: I^{r}(J) \rightarrow J$, which is an $R$-space of type $\left(C_{n-1},\left\{\alpha_{r}\right\}\right)($ cf. $\S 4$ in [24]). 
2.2. IG manifold $\left(W ; C^{*}, N\right)$ of corank $r$. Let $W$ be a submanifold of $I^{r}(J)$ satisfying the following condition:

$$
(W .0) \quad q: W \rightarrow J: \quad \text { submersion, }
$$

where $q=\left.\pi\right|_{W}$ and $\pi: I^{r}(J) \rightarrow J$ is the projection. From the two differential systems $\bar{C}$ and $\bar{N}$ on $I^{r}(J)$, we obtain the differential systems $C^{*}$ and $N$ on $W$ by restricting $\bar{C}$ and $\bar{N}$ to $W$. At each point $w \in W$, we define the bilinear map $\gamma_{w}: N(w) \times N(w) \rightarrow \mathfrak{t}_{-3}(w)=T_{w}(W) / C^{*}(w)$ as follows; For vectors $X$ and $Y$ in $N(w)$, let $\tilde{X} \in \Gamma(N)$ and $\tilde{Y} \in \Gamma(N)$ be vectorfields such that $X=(\tilde{X})_{w}$ and $Y=(\tilde{Y})_{w}$, where $\Gamma(N)$ denotes the space of sections of $N$. Then $\gamma_{w}$ is defined by

$$
\gamma_{w}(X, Y)=\pi_{-3}\left([\tilde{X}, \tilde{Y}]_{w}\right)
$$

where $\pi_{-3} ; T_{w}(W) \rightarrow \mathfrak{t}_{-3}(w)=T_{w}(W) / C^{*}(w)$ is the projection. Let $w_{o}$ be an arbitrary point of $W$. We will introduce a coordinate system $\left(x_{i}, x_{\alpha}, z, p_{i}, p_{\alpha}, a_{i}^{\alpha}, b_{i}^{\alpha}, s_{i j}\right)(1 \leqq i \leqq j \leqq r, r+1 \leqq$ $\alpha \leqq n)$ of $I^{r}(J)$ on a neighborhood $\bar{U}$ of $w_{o}$ as in $\S 2.1$. Then, by the condition (W.0), 1 -forms $\left\{\varpi, \varpi_{i}^{*}, \varpi_{\alpha}^{*}, \omega^{\alpha}, d x_{i}(1 \leqq i \leqq r, r+1 \leqq \alpha \leqq n)\right\}$ remain linearly independent at each point of $W \cap \bar{U}$, when restricted to the submanifold $W$. Hence we have

$$
C^{*}=\{\varpi=0\}, \quad N=\left\{\varpi=\varpi_{1}^{*}=\cdots=\varpi_{r}^{*}=0\right\},
$$

where we denote the restricted 1-forms on $W$ by the same symbols as those on $\bar{U}$. The contact form $\varpi$ fixes the basis $Z$ of $\mathfrak{t}_{-3}(w)$ by $Z=\pi_{-3}(\hat{Z}), \hat{Z} \in T_{w}(W)$ such that $\varpi(\hat{Z})=1$, Then we have

$$
\gamma_{w}(X, Y)=\varpi\left([\tilde{X}, \tilde{Y}]_{w}\right) \cdot Z=-d \varpi(X, Y) \cdot Z,
$$

which shows that $\gamma_{w}(X, Y)$ is well-defined for $X, Y \in N(w)$ (cf. $§ 3.1$ [27]). Moreover, from (2.1), we have

$$
d \varpi=\sum_{i=1}^{r} d x_{i} \wedge \varpi_{i}^{*}+\sum_{\alpha=r+1}^{n} \omega^{\alpha} \wedge \varpi_{\alpha}^{*} \equiv \sum_{\alpha=r+1}^{n} \omega^{\alpha} \wedge \varpi_{\alpha}^{*} \quad\left(\bmod \quad \varpi, \varpi_{1}^{*}, \ldots, \varpi_{r}^{*}\right) .
$$

Hence we have

$$
\begin{aligned}
\operatorname{Ch}\left(C^{*}\right)=\left\{\varpi=\varpi_{i}^{*}=\varpi_{\alpha}^{*}=\omega^{\alpha}\right. & \left.=d x_{i}=0 \quad(1 \leqq i \leqq r, r+1 \leqq \alpha \leqq n)\right\} \\
& =\left\{d z=d p_{1}=\cdots=d p_{n}=d x_{1}=\cdots=d x_{n}=0\right\}=\operatorname{Ker} p_{*}
\end{aligned}
$$

and

$$
\begin{aligned}
\operatorname{Ker} \gamma_{w}=\left\{X \in N(w) \mid \gamma_{w}(X, Y)\right. & =0 \quad \text { for } \quad \forall Y \in N(w)\} \\
& =\left\{\varpi=\varpi_{i}=\varpi_{\alpha}^{*}=\omega^{\alpha}=0 \quad(1 \leqq i \leqq r, r+1 \leqq \alpha \leqq n)\right\}
\end{aligned}
$$

Thus $N^{\perp}=\operatorname{Ker} \gamma=\bigcup_{w \in W} \operatorname{Ker} \gamma_{w}$ is a subbundle of $N$ which coincides with the restriction to W of $\bar{N}^{\perp}$ on $I^{r}(J)$. Summarizing, $\left(W ; C^{*}, N\right)$ satisfies the following conditions:

(W.1) $\quad C^{*}$ and $N$ are differential systems of codimension 1 and $r+1$ respectively such that $N \subset C^{*}$.

(W.2) $\quad C h\left(C^{*}\right)$ is a subbundle of $N$ of codimension $2 n-r$.

$(W .3) \quad \operatorname{rank} \gamma_{w}=2(n-r)$ at each $w \in W$.

$(W .4) \quad \operatorname{Ch}\left(C^{*}\right)(w) \cap \operatorname{Ch}(N)(w)=\{0\}$ at each $w \in W$.

In fact the last condition ( $W .4)$ follows from the Realization Lemma for $(W, N, q, J)$ (see $\S 4.1$ $[27])$. The condition (W.3) is equivalent to the following condition:

$\left(W .3^{\prime}\right) \quad N^{\perp}=\operatorname{Ker} \gamma$ is a subbundle of $N$ of codimension $2(n-r)$.

Conversely these four conditions, at least locally, characterize submanifolds in $I^{r}(J)$ satisfying $(W .0)$ as in the following. 
We call the triplet $\left(W ; C^{*}, N\right)$ of a manifold and two differential systems on it an $I G$ ( Involutive Grassmann) manifold of corank $r$ if these satisfy the above four conditions $(W .1)$ to $(W .4)$. We have the (local) Realization Theorem for $I G$ manifolds as follows: From conditions $(W .1)$ and $(W .2)$, the codimension of the foliation defined by $\mathrm{Ch}\left(C^{*}\right)$ is $2 n+1$. Assume that $W$ is regular with respect to $\mathrm{Ch}\left(C^{*}\right)$, i.e., the space $J=W / \mathrm{Ch}\left(C^{*}\right)$ of leaves of this foliation is a manifold of dimension $2 n+1$ such that each fibre of the projection $q$ : $W \rightarrow J=W / \mathrm{Ch}\left(C^{*}\right)$ is connected and $q$ is a submersion. Then $C^{*}$ drops down to $J$. Namely there exists a differential system $C$ on $J$ of codimension 1 such that $C^{*}=q_{*}^{-1}(C)$. From $\mathrm{Ch}(C)=\{0\},(J, C)$ becomes a contact manifold of dimension $2 n+1$. Conditions $(W .2)$ and $(W .3)$ imply that the image of the following map $\iota$ is an involutive subspace of codimension $r$ in the symplectic vector space $(C(u), d \varpi)$ :

$$
\iota(w)=q_{*}(N(w)) \subset C(u), \quad u=q(w) .
$$

Namely $\iota$ is a map from $W$ into $I^{r}(J)$, which is an immersion by the Realization Lemma for $(W, N, q, J)$ and (W.4). Moreover, similarly as in Theorem 4.1 [27], we obtain

Theorem 2.1. Let $\left(W ; C^{*}, N\right)$ and $\left(\hat{W} ; \hat{C}^{*}, \hat{N}\right)$ be $I G$ manifolds of corank $r$. Assume that $W$ and $\hat{W}$ are regular with respect to $C h\left(C^{*}\right)$ and $C h\left(\hat{C}^{*}\right)$ respectively. Let $(J, C)$ and $(\hat{J}, \hat{C})$ be the associated contact manifolds. Then an isomorphism $\Phi:\left(W ; C^{*}, N\right) \rightarrow\left(\hat{W} ; \hat{C}^{*}, \hat{N}\right)$ induces a contact diffeomorphism $\varphi:(J, C) \rightarrow(\hat{J}, \hat{C})$ such that the following commutes;

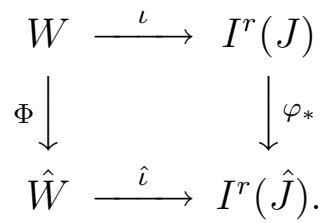

By this theorem, the submanifold theory for $I^{r}(J)$ is reformulated as the geometry of $I G$ manifolds of corank $r$.

2.3. Covariant systems of $\left(W ; C^{*}, N\right)$. Let $\left(W ; C^{*}, N\right)$ be an $I G$ manifold of corank $r$. In this subsection, we will consider several covariant systems of $\left(W ; C^{*}, N\right)$.

(1) Covariant system $N^{\perp}$.

$N^{\perp}$ is defined by $N^{\perp}(w)=\operatorname{Ker} \gamma_{w}$ at each $w \in W$. Hence, by (W.3), $N^{\perp}$ is a subbundle of $N$ of codimension $2(n-r)$ and contains $\mathrm{Ch}\left(C^{*}\right)$ as a subbundle of codimension $r$. In fact, utilizing the local Realization Theorem for $\left(W ; C^{*}, N\right)$ and canonical coordinate system as in $\S 2.1$, we have

$$
N=\left\{\varpi=\varpi_{1}^{*}=\cdots=\varpi_{r}^{*}=0\right\}, \quad N^{\perp}=\left\{\varpi=\varpi_{i}^{*}=\varpi_{\alpha}^{*}=\omega^{\alpha}=0 \quad(1 \leqq i \leqq r, r+1 \leqq \alpha \leqq n)\right\} .
$$

Hence we see, from (2.2), $\mathrm{Ch}(N)(w) \subset N^{\perp}(w)$ at each $w \in W$. Moreover we see, from (2.1), $\mathrm{Ch}\left(C^{*}\right)(w) \cap \mathrm{Ch}\left(N^{\perp}\right)(w)=\{0\}$ at each $w \in W$.

(2) Covariant system $N^{*}=\partial N^{\perp}+N$ and the weak symbol algebra $\mathfrak{t}(w)$ of $\left(W ; C^{*}, N\right)$.

$N^{*}$ is defined by $N^{*}(w)=\partial N^{\perp}(w)+N(w)$ at each $w \in W$, where $\partial N^{\perp}$ denotes the derived system of $N^{\perp}$. From $d \varpi \equiv 0 \quad\left(\bmod \varpi, \varpi_{1}^{*}, \ldots, \varpi_{n}^{*}\right)$, it follows that $\partial N^{\perp} \subset C^{*}$. In the following, we will assume that $N^{*}$ is a subbundle of $T(W)$, hence of $C^{*}$, which contains $N$. Here $N^{\perp}$ and $C^{*}$ are subbundles of $T(W)$ such that $\partial N^{\perp} \subset C^{*}$. Hence subbundles $N^{\perp}, C^{*}$ and $T(W)$ define a filtration on $W$. Namely, putting $T^{-1}=N^{\perp}, T^{-2}=C^{*}, T^{p}=T(W)$ for $p \leqq-3$, we have

$$
\left[\mathcal{T}^{p} \cdot \mathcal{T}^{q}\right] \subset \mathcal{T}^{p+q} \quad \text { for } \quad p, q<0
$$


where $\mathcal{T}^{p}=\Gamma\left(T^{p}\right)$. We can form the weak symbol algebra $\mathfrak{t}(w)$ of $\left(W ; C^{*}, N\right)$ at $w \in W$ as follows: Put $\mathfrak{t}_{-3}(w)=T_{w}(W) / C^{*}(w), \mathfrak{t}_{-2}(w)=C^{*}(w) / N^{\perp}(w)$ and $\mathfrak{t}_{-1}(w)=N^{\perp}(w)$. Then as in the symbol algebra of $P D$ manifolds, we can introduce the Lie brackets in

$$
\mathfrak{t}(w)=\mathfrak{t}_{-3}(w) \oplus \mathfrak{t}_{-2}(w) \oplus \mathfrak{t}_{-1}(w),
$$

as follows; For $X \in \mathfrak{t}_{p}(w)$ and $Y \in \mathfrak{t}_{q}(w)$, let us take $\tilde{X} \in \mathcal{T}^{p}$ and $\tilde{Y} \in \mathcal{T}^{q}$ such that $X=$ $\pi_{p}\left((\tilde{X})_{w}\right)$ and $Y=\pi_{q}\left((\tilde{Y})_{w}\right)$, where $\pi_{p}: T^{p}(w) \rightarrow \mathfrak{t}_{p}(w)$ is the projection. Then the bracket product is defined by

$$
[X, Y]=\pi_{p+q}\left([\tilde{X}, \tilde{Y}]_{w}\right) \in \mathfrak{t}_{p+q}(w) .
$$

Now $N$ defines a subspace $\mathfrak{t}_{N}(w)=N(w) / N^{\perp}(w)$ of $\mathfrak{t}_{-2}(w)$. Then $\gamma_{w}$ induces the nondegenerate paing $\hat{\gamma}_{w}: \mathfrak{t}_{N}(w) \times \mathfrak{t}_{N}(w) \rightarrow \mathfrak{t}_{-3}(w)$. Moreover, in Lie brackets of $\mathfrak{t}(w)$, we have

$$
\left[\mathfrak{t}_{-1}(w), \mathfrak{t}_{N}(w)\right]=0,
$$

which is equivalent to $\left[\Gamma\left(N^{\perp}\right), \Gamma(N)\right] \subset \Gamma\left(C^{*}\right)$. Namely $\mathfrak{t}_{N}(w)$ is an abelian ideal of $\mathfrak{t}(w)$. Thus we can form the quotient Lie algebra

$$
\tilde{\mathfrak{t}}(w)=\mathfrak{t}(w) / \mathfrak{t}_{N}(w)=\mathfrak{t}_{-3}(w) \oplus \tilde{\mathfrak{t}}_{-2}(w) \oplus \mathfrak{t}_{-1}(w),
$$

where $\tilde{\mathfrak{t}}_{-2}(w)=C^{*}(w) / N(w)$. Conversely, for $Y \in \mathfrak{t}_{-2}(w),\left[Y, \mathfrak{t}_{-1}(w)\right]=0$ implies $Y \in \mathfrak{t}_{N}(w)$, which can be checked as follows; Take a vector field $\tilde{Y} \in \Gamma\left(C^{*}\right)$ such that $\pi_{-2}\left((\tilde{Y})_{w}\right)=Y$. Then, from (2.2), we see that $\left[\tilde{Y}, \Gamma\left(N^{\perp}\right)\right] \subset \Gamma\left(C^{*}\right)$ iff

$$
\tilde{Y}\rfloor d \varpi \equiv-\sum_{i=1}^{r} \varpi_{i}^{*}(\tilde{Y}) d x_{i} \equiv 0 \quad\left(\bmod N^{\perp}\right) \quad \Longleftrightarrow \quad \varpi_{i}^{*}(\tilde{Y})=0 \quad \text { for } i=1, \ldots, r,
$$

which implies $\tilde{Y} \in \Gamma(N)$. Thus, in the Lie algebra $\tilde{\mathfrak{t}}(w)$, we have

(a.1) If $\bar{Y} \in \tilde{\mathfrak{t}}_{-2}(w)$ and $\left[\bar{Y}, \mathfrak{t}_{-1}(w)\right]=0$, then $\bar{Y}=0$.

(a.2) $\quad \operatorname{Ch}\left(C^{*}\right)(w)=\left\{X \in \mathfrak{t}_{-1}(w) \mid\left[X, \tilde{\mathfrak{t}}_{-2}(w)\right]=0\right\}$.

Here $(a .2)$ can be checked as follows; Take a vector field $\tilde{X} \in \Gamma\left(N^{\perp}\right)$ such that $(\tilde{X})_{w}=$ $X \in \mathfrak{t}_{-1}(w)=N^{\perp}(w)$. Then $\left[X, \tilde{\mathfrak{t}}_{-2}(w)\right]=0$ iff $\left[\tilde{X}, \Gamma\left(C^{*}\right)\right] \subset \Gamma\left(C^{*}\right)$, which is equivalent to $\tilde{X} \in \Gamma\left(\mathrm{Ch}\left(C^{*}\right)\right)$. By $(a .1)$ and $(a .2)$, the Lie bracket in $\tilde{\mathfrak{t}}(w)$ induces the non-degenerate paring

$$
\tilde{\gamma}_{w}: E(w) \times \tilde{\mathfrak{t}}_{-2}(w) \rightarrow \mathfrak{t}_{-3}(w),
$$

where $E(w)=\mathfrak{t}_{-1}(w) / \operatorname{Ch}\left(C^{*}\right)(w)$. Thus $\tilde{\mathfrak{t}}_{-2}(w) \cong \mathfrak{t}_{-3}(w) \otimes E(w)^{*}$.

(3) Covariant systems $H(N)$ and $S(N)$.

Here we define, at each $w \in W$,

$$
H(N)(w)=\left\{X \in N^{\perp}(w) \mid\left[X, \mathfrak{t}_{-1}(w)\right]=0 \text { in } \tilde{\mathfrak{t}}(w)\right\}=\left\{X \in N^{\perp}(w) \mid\left[X, \Gamma\left(N^{\perp}\right)\right] \subset \Gamma(N)\right\},
$$

and

$$
S(N)(w)=H(N)(w) \cap \mathrm{Ch}\left(C^{*}\right)(w)=\left\{X \in \mathrm{Ch}\left(C^{*}\right)(w) \mid\left[X, \Gamma\left(N^{\perp}\right)\right] \subset \Gamma(N)\right\} .
$$

Utilizing the canonical coordinate system as in $\S 2.2$, we have, for $X \in \mathrm{Ch}\left(C^{*}\right)(w)$,

$$
X\rfloor d \varpi \equiv 0, \quad X\rfloor d \varpi_{i}^{*} \equiv-\sum_{j=1}^{r} \varpi_{i j}(X) d x_{j} \quad\left(\bmod \quad N^{\perp}\right)
$$

Hence we have

$$
S(N)(w)=\left\{\varpi=\varpi_{i}^{*}=\varpi_{\alpha}^{*}=\omega^{\alpha}=d x_{i}=\varpi_{i j}=0 \quad(1 \leqq i \leqq j \leqq r, r+1 \leqq \alpha \leqq n)\right\} .
$$


Now, for a vector $f$ of $\mathrm{Ch}\left(C^{*}\right)(w)$, we have a map $\kappa: \mathrm{Ch}\left(C^{*}\right)(w) \rightarrow \mathfrak{t}_{-3}(w) \otimes S^{2}\left(E(w)^{*}\right)$ by taking brackets in $\tilde{\mathfrak{t}}(w)$

$$
\kappa(f)(\bar{X}, \bar{Y})=[[f, X], Y]=[[f, Y], X] \in \mathfrak{t}_{-3}(w),
$$

for $X, Y \in \mathfrak{t}_{-1}(w)$ such that $\bar{X}=\pi_{-1}(X), \bar{Y}=\pi_{-1}(Y)$, where $[f,[X, Y]]=0$ and $\pi_{-1}$ : $\mathfrak{t}_{-1}(w) \rightarrow E(w)$ is the projection. Then $\kappa$ induces the injective map

$$
\hat{\kappa}: \operatorname{Ch}\left(C^{*}\right)(w) / S(N)(w) \rightarrow \mathfrak{t}_{-3}(w) \otimes S^{2}\left(E(w)^{*}\right) .
$$

Moreover, when $S(N)$ forms a subbundle, we have an injective map

$$
\gamma_{c}: S(N)(w) \rightarrow \mathfrak{t}_{N}(w) \otimes E(w)^{*}
$$

as follows; For vectors $X \in S(N)(w)$ and $Y \in N^{\perp}(w)$, we take $\tilde{X} \in \Gamma(S(N))$ and $\tilde{Y} \in \Gamma\left(N^{\perp}\right)$ such that $X=\tilde{X}_{w}$ and $Y=\tilde{Y}_{w}$. Then $[\tilde{X}, \tilde{Y}] \in \Gamma(N)$ and $[\tilde{X}, \tilde{Y}] \in \Gamma\left(\operatorname{Ch}\left(C^{*}\right)\right)$ when $\tilde{Y} \in$ $\Gamma\left(\operatorname{Ch}\left(C^{*}\right)\right)$. From $[f \tilde{X}, g \tilde{Y}]=f g[\tilde{X}, \tilde{Y}]+f(\tilde{X}(g)) Y-g(\tilde{Y}(f)) X$, we see

$$
\hat{\gamma}_{c}(X, Y)=\pi_{N}\left([\tilde{X}, \tilde{Y}]_{w}\right) \in \mathfrak{t}_{N}(w)=N(w) / N^{\perp}(w)
$$

depends only on $X$ and $Y$ and $\hat{\gamma}_{c}(X, Y)=0$ if $Y \in \operatorname{Ch}\left(C^{*}\right)(w)$, where $\pi_{N}: N(w) \rightarrow \mathfrak{t}_{N}(w)$ is the projection. Thus $\hat{\gamma}_{c}$ induces the map $\gamma_{c}: S(N)(w) \rightarrow \mathfrak{t}_{N}(w) \otimes E(w)^{*}$. Assume that $\gamma_{c}(X)=0$. This implies that $\hat{\gamma}_{c}(X, Y)=\pi_{N}\left([\tilde{X}, \tilde{Y}]_{w}\right)=0$ for any $Y \in N^{\perp}(w)$. Hence we have $\left[X, \Gamma\left(N^{\perp}\right)\right] \subset \Gamma\left(N^{\perp}\right)$, i.e., $X \in \operatorname{Ch}\left(N^{\perp}\right)(w)$. Thus $X=0$ follows from $\operatorname{Ch}\left(C^{*}\right)(w) \cap$ $\mathrm{Ch}\left(N^{\perp}\right)(w)=\{0\}$ at each $w \in W$, which shows that $\gamma_{c}$ is injective.

Now we prepare the following proposition for the later use in $\S 7.2$. We assume the following compatibility condition for $\left(W ; C^{*}, N\right)$;

$\left(C^{*}\right) \quad$ There exists, at each $w \in W$, an $n$-dimensional integral element $E$ of $(W, N)$ such that

$$
E \cap \operatorname{Ch}\left(C^{*}\right)(w)=\{0\} .
$$

Then, $\pi_{N}(E)$ is an isotropic subspace of the symplectic vector space $\left(\mathfrak{t}_{N}(w), \hat{\gamma}_{W}\right)$, where $\pi_{N}$ : $N(w) \rightarrow \mathfrak{t}_{N}(w)=N(w) / N^{\perp}(w)$ is the projection. Hence $E^{\prime}=E \cap N^{\perp}(w)$ is an $r$-dimensional subspace such that

$$
\mathfrak{t}_{-1}(w)=N^{\perp}(w)=E^{\prime} \oplus \operatorname{Ch}\left(C^{*}\right)(w) \quad \text { and } \quad\left[E^{\prime}, E^{\prime}\right]=0 \quad \text { in } \quad \tilde{\mathfrak{t}}(w) .
$$

We have (cf. [27] Proposition 4.1)

Proposition 2.1. Let $\left(W ; C^{*}, N\right)$ be an $I G$ manifold of corank $r$ satisfying the condition $\left(C^{*}\right)$ above. Then $\operatorname{dim} C^{*}(w) / N^{*}(w)=\operatorname{dim} H(N)(w) / S(N)(w)$ at each $w \in W$.

Proof. We utilize the above decomposition of $N^{\perp}(w)=E^{\prime} \oplus \operatorname{Ch}\left(C^{*}\right)(w)$. For $X \in H(w)$, we decompose $X=v_{X}+A_{X}$, where $v_{X} \in E^{\prime}$ and $A_{X} \in \operatorname{Ch}\left(C^{*}\right)(w)$. From $\left[X, \mathfrak{t}_{-1}(w)\right]=0$, we have $[X, v]=\left[A_{X}, v\right]=0$ for $\forall v \in E^{\prime}$ and $[X, A]=\left[v_{X}, A\right]=0$ for $\forall A \in \operatorname{Ch}\left(C^{*}\right)(w)$. Hence $A_{X} \in S(N)(w)$ and we get

$$
H(N)(w)=E^{\prime \prime} \oplus S(N)(w),
$$

where $E^{\prime \prime}=E^{\prime} \cap H(N)(w)=\left\{v \in E^{\prime} \mid[v, A]=0\right.$ for $\left.\forall A \in \operatorname{Ch}\left(C^{*}\right)(w)\right\}$.

On the other hand, $N^{*}(w)$ defines the subspace $\mathfrak{n}^{*}(w)=\tilde{\pi}_{-2}\left(N^{*}(w)\right) \subset \tilde{\mathfrak{t}}_{-2}(w)=C^{*}(w) / N(w)$. In fact $\mathfrak{n}^{*}(w)=\left[\mathfrak{t}_{-1}(w), \mathfrak{t}_{-1}(w)\right]$ in $\tilde{\mathfrak{t}}(w)$. This subspace $\mathfrak{n}^{*}(w)$ defines the subspace $F$ of $E(w)^{*}$ via the non-degenerate paring $\tilde{\gamma}_{w}$. Identifying $E^{\prime}$ with $E(w)=N^{\perp}(w) / \mathrm{Ch}\left(C^{*}\right)(w)$, let $F^{\perp} \subset E^{\prime}$ be the annihilator of $F$. Then we get $\left[v, \mathfrak{n}^{*}(w)\right]=0$ for $v \in F^{\perp}$ and we calculate

$$
[v,[\hat{v}, A]]=[\hat{v},[v, A]]=0 \quad \text { for } \quad \forall \hat{v} \in E^{\prime}, \forall A \in \operatorname{Ch}\left(C^{*}\right)(w) .
$$

Hence, by $(a .1)$ and $(a .2)$, we obtain $[v, A]=0$ for $\forall A \in \operatorname{Ch}\left(C^{*}\right)(w)$, which shows $F^{\perp}=E^{\prime \prime}$. This completes the proof of Proposition. 


\section{IG MANIFOLDS INDUCED FROM PD MANIFOLDS OF SECOND ORDER}

3.1. Covariant Systems associated with a $P D$ manifold of second order. Let $\left(R ; D^{1}, D^{2}\right)$ be a $P D$ manifold of second order. We will assume the regularity of $\left(R ; D^{1}, D^{2}\right)$ in terms of the symbol algebras as follows; Let $\mathfrak{c}^{2}(n)=\mathfrak{c}_{-3} \oplus \mathfrak{c}_{-2} \oplus \mathfrak{c}_{-1}$ be the symbol algebra of $(L(J), E)$ given by

$$
\mathfrak{c}_{-3}=\mathbb{R}, \quad \mathfrak{c}_{-2}=V^{*}, \quad \mathfrak{c}_{-1}=V \oplus S^{2}\left(V^{*}\right),
$$

where $\operatorname{dim} V=n$ and $\operatorname{dim} J=2 n+1$ (cf. $\S 2.5$ [27]). Let $\mathfrak{f}$ be a fixed subspace of $S^{2}\left(V^{*}\right)$ and $\mathfrak{s}$ be a subalgebra of $\mathfrak{c}^{2}(n)$, which is defined by

$$
\mathfrak{s}_{-3}=\mathbb{R}, \quad \mathfrak{s}_{-2}=V^{*}, \quad \mathfrak{s}_{-1}=V \oplus \mathfrak{f}, \quad \mathfrak{f} \subset S^{2}\left(V^{*}\right),
$$

$\left(R ; D^{1}, D^{2}\right)$ is called regular of type $\mathfrak{s}$ iff the symbol algebra $\mathfrak{s}(v)$ of $\left(R ; D^{1}, D^{2}\right)$ at each $v \in R$ is isomorphic to $\mathfrak{s}$ (cf. [20],[23], $\S 1$ [27]). We note here that $R$ satisfies the compatibility condition $(C)$ :

$$
\text { (C) } \quad p^{(1)}: R^{(1)} \rightarrow R \text { is onto. }
$$

where $R^{(1)}$ is the first prolongation of $\left(R ; D^{1}, D^{2}\right)$ (cf. $\left.\S 4.2[27]\right)$.

Here we first recall, from $\S 3$ in [20], the structure of the group $G\left(\mathfrak{c}^{2}(n)\right)$ of graded Lie algebra automorphisms of $\mathfrak{c}^{2}(n)$. Let $\kappa: \mathfrak{c}_{-1} \rightarrow V_{c}=\mathfrak{c}_{-1} / \mathfrak{F}, \mathfrak{F}=S^{2}\left(V^{*}\right) \subset \mathfrak{c}_{-1}$, be the projection. Then $\kappa_{0}=\left.\kappa\right|_{V}$ is a linear isomorphism of $V$ onto $V_{c}$. Since $\mathfrak{F}=\left\{X \in \mathfrak{c}_{-1} \mid\left[X, \mathfrak{c}_{-2}\right]=0\right\}$, it follows that $\phi(\mathfrak{F})=\mathfrak{F}$ for $\phi \in G\left(\mathfrak{c}^{2}(n)\right)$. Hence $\phi$ induces the linear isomorphism $\hat{\phi}: V_{c} \rightarrow V_{c}$ such that $\hat{\phi} \cdot \kappa=\kappa \cdot \phi$. We define the closed normal subgroup $N\left(\mathfrak{c}^{2}(n)\right)$ of $G\left(\mathfrak{c}^{2}(n)\right)$ by setting

$$
N\left(\mathfrak{c}^{2}(n)\right)=\left\{\phi \in G\left(\mathfrak{c}^{2}(n)\right)|\phi|_{\mathfrak{c}_{-3}}=i d_{\mathfrak{c}_{-3}} \text { and } \hat{\phi}=i d_{V_{c}}\right\} .
$$

We define the homomorphism $\chi: G L(V) \times G L(\mathbb{R}) \rightarrow G\left(\mathfrak{c}^{2}(n)\right)$, for $a \in G L(V)$ and $b \in$ $G L(\mathbb{R})=\mathbb{R}^{\times}$, by putting

$\left.\chi(a, b)\right|_{V}=a,\left.\quad \chi(a, b)\right|_{\mathfrak{c}_{-3}}=b \cdot i d_{\mathfrak{c}_{-3}},\left.\quad \chi(a, b)\right|_{V^{*}}=b \cdot\left(a^{*}\right)^{-1} \quad$ and $\left.\quad \chi(a, b)\right|_{S^{2}\left(V^{*}\right)}=b \cdot \otimes^{2}\left(a^{*}\right)^{-1}$, where $a^{*}$ is the adjoint linear map of $a$. We put $G_{0}\left(\mathfrak{c}^{2}(n)\right)=\chi(G L(V) \times G L(\mathbb{R}))$. Moreover, let $S\left(\mathfrak{c}^{2}(n)\right)$ be the set of abelian subalgebras $\hat{V}$ of $\mathfrak{c}^{2}(n)$ such that $\mathfrak{c}_{-1}=\hat{V} \oplus \mathfrak{F}$ (direct sum). Then we have (Proposition 3.7 [20])

(1) $N\left(\mathfrak{c}^{2}(n)\right)$ is canonically isomorphic to the vector group $S^{3}\left(V^{*}\right)$. Furthermore $N\left(\mathfrak{c}^{2}(n)\right)$ acts simply transitively on $S\left(\mathfrak{c}^{2}(n)\right)$.

(2) $G_{0}\left(\mathfrak{c}^{2}(n)\right)=\left\{\phi \in G\left(\mathfrak{c}^{2}(n)\right) \mid \phi(V)=V\right\}$ and $G\left(\mathfrak{c}^{2}(n)\right)=G_{0}\left(\mathfrak{c}^{2}(n)\right) \cdot N\left(\mathfrak{c}^{2}(n)\right)$ is the semi-direct product.

The action of $N\left(\mathfrak{c}^{2}(n)\right)$ can be explicitly described as follows: First we identify

$$
\left.\left.S^{3}\left(V^{*}\right) \cong\left\{\rho: V \rightarrow S^{2}\left(V^{*}\right) \mid v_{1}\right\rfloor \rho\left(v_{2}\right)=v_{2}\right\rfloor \rho\left(v_{1}\right)\right\} .
$$

Then, for $\rho \in S^{3}\left(V^{*}\right)$, we define the element $A_{\rho} \in N\left(\mathfrak{c}^{2}(n)\right)$ by

$$
\left.A_{\rho}\right|_{\mathfrak{c}_{-3}}=i d_{\mathfrak{c}_{-3}},\left.\quad A_{\rho}\right|_{V^{*}}=i d_{V^{*}},\left.\quad A_{\rho}\right|_{\mathfrak{F}}=i d_{\mathfrak{F}} \quad \text { and }\left.\quad A_{\rho}\right|_{V}=i d_{V}+\rho .
$$

Let $G(\mathfrak{s})$ be the group of graded Lie algebra automorphisms of $\mathfrak{s}$. Then $G(\mathfrak{s})$ is a subgroup of $G\left(\mathfrak{c}^{2}(n)\right)$. In fact, we have (Corollary 5.8 [20])

$$
G(\mathfrak{s})=\left\{\sigma \in G\left(\mathfrak{c}^{2}(n)\right) \mid \sigma(\mathfrak{s})=\mathfrak{s}\right\}=\left\{\sigma \in G\left(\mathfrak{c}^{2}(n)\right) \mid \sigma\left(\mathfrak{s}_{-1}\right)=\mathfrak{s}_{-1} \subset \mathfrak{c}_{-1}\right\} .
$$

Thus $G(\mathfrak{s})$ is a semi-direct product $G_{0}(\mathfrak{s}) \cdot N(\mathfrak{s})$, where

$$
\begin{aligned}
G_{0}(\mathfrak{s}) & =\{\sigma \in G(\mathfrak{s}) \mid \sigma(V)=V\}=\left\{\sigma \in G_{0}\left(\mathfrak{c}^{2}(n)\right) \mid \sigma(\mathfrak{f})=\mathfrak{f} \subset \mathfrak{F}=S^{2}\left(V^{*}\right)\right\}, \\
N(\mathfrak{s}) & =\left\{\sigma \in N\left(\mathfrak{c}^{2}(n)\right) \mid \sigma\left(\mathfrak{s}_{-1}\right)=\mathfrak{s}_{-1}\right\}=\left\{A_{\rho} \in N\left(\mathfrak{c}^{2}(n)\right) \mid \rho(V) \subset \mathfrak{f}\right\} \cong \mathfrak{f}^{(1)} .
\end{aligned}
$$

Here $\mathfrak{f}^{(1)}$ denotes the prolongation of $\mathfrak{f}$ (see $\S 5[20]$ for the detail). 
Now, starting from an invariant subspace $E \subset V$ of $G_{0}(\mathfrak{s})$, we will construct the first order covariant system $\tilde{N}=\tilde{N}(E)$ of $\left(R ; D^{1}, D^{2}\right)$ as in the following; Let $E^{\perp} \subset V^{*}$ be the annihilator subspace of $E$. Then $E^{\perp}$ is an $G(\mathfrak{s})$-invariant subspace of $\mathfrak{s}_{-2}=V^{*}$. Let $v$ be any point of $R$ and let $\mathfrak{s}(v)$ be the symbol algebra at $v \in R$. Take a graded Lie algebra isomorphism $\phi$ of $\mathfrak{s}(v)$ onto $\mathfrak{s}$. Let $\mathfrak{n}(E)(v)$ denote the linear subspace of $\mathfrak{s}_{-2}(v)$ defined by

$$
\mathfrak{n}(E)(v)=\phi^{-1}\left(E^{\perp}\right) .
$$

Then, since $E^{\perp}$ is $G(\mathfrak{s})$-invariant, it follows that $\mathfrak{n}(E)(v)$ is well-defined. We define the linear subspace $\tilde{N}(E)(v)$ of $D^{1}(v)$ by setting

$$
\tilde{N}(E)(v)=\left(\pi_{-2}\right)^{-1}(\mathfrak{n}(E)(v)),
$$

where $\pi_{-2}: D^{1}(v) \rightarrow \mathfrak{s}_{-2}(v)=D^{1}(v) / D^{2}(v)$ is the projection. Then it follows that the assignment $v \mapsto \tilde{N}(E)(v)$ defines a subbundle $\tilde{N}=\tilde{N}(E)$ of $D^{1}$, which contains $D^{2}$.

Moreover we will define the covariant systems $\tilde{N}^{\perp}=\tilde{N}^{\perp}(E)$ and $\tilde{N}^{*}=\tilde{N}^{*}(E)$ of $\left(R ; D^{1}, D^{2}\right)$ as follows; Take a graded Lie algebra isomorphism $\phi$ of $\mathfrak{s}(v)$ onto $\mathfrak{s}$. Let $\tilde{N}^{\perp}(E)(v)$ denote the linear subspace of $D^{2}(v)=\mathfrak{s}_{-1}(v)$ defined by

$$
\tilde{N}^{\perp}(E)(v)=\phi^{-1}(E \oplus \mathfrak{f}) \subset D^{2}(v),
$$

Then, since $E \oplus \mathfrak{f}$ is $G(\mathfrak{s})$-invariant, it follows that $\tilde{N}^{\perp}(E)(v)$ is well-defined. Thus the assignment $v \rightarrow \tilde{N}^{\perp}(E)(v)$ defines a subbundle $\tilde{N}^{\perp}=\tilde{N}^{\perp}(E)$ of $D^{2}$.

Then $\tilde{N}^{*}=\tilde{N}^{*}(E)$ is defined by $\tilde{N}^{*}(E)(v)=\partial \tilde{N}^{\perp}(E)(v)+\tilde{N}(E)(v)$ at each $v \in R$, where $\partial \tilde{N}^{\perp}(E)$ denotes the derived system of $\tilde{N}^{\perp}(E)$. From $\tilde{N}^{\perp}(E) \subset D^{2} \subset \tilde{N}(E)$ and $\partial D^{2} \subset D^{1}$, it follows that $\partial \tilde{N}^{\perp}(E) \subset D^{1}$ and $D^{2} \subset \tilde{N}^{*}(E) \subset D^{1}$. In terms of the symbol algebra, we calculate $[E \oplus \mathfrak{f}, E \oplus \mathfrak{f}]=E\rfloor \mathfrak{f}$. Hence $\tilde{N}^{*}(E)$ corresponds to the subspace $\left.E\right\rfloor \mathfrak{f}+E^{\perp}$ of $\mathfrak{s}_{-2}=V^{*}$. We put

$$
\hat{E}=E \cap(E\rfloor \mathfrak{f})^{\perp}=\left\{v \in E \mid v \odot E \subset \mathfrak{f}^{\perp}\right\} .
$$

Then $\tilde{N}^{*}(E)$ coincides with the first order covariant system corresponding to the $G_{0}(\mathfrak{s})$-invariant subspace $\hat{E}$. Namely take a graded Lie algebra isomorphism $\phi$ of $\mathfrak{s}(v)$ onto $\mathfrak{s}$. Let $\mathfrak{n}^{*}(E)(v)$ denote the linear subspace of $\mathfrak{s}_{-2}(v)$ defined by

$$
\mathfrak{n}^{*}(E)(v)=\phi^{-1}\left((\hat{E})^{\perp}\right) .
$$

Then we have

$$
\tilde{N}^{*}(E)(v)=\left(\pi_{-2}\right)^{-1}\left(\mathfrak{n}^{*}(E)(v)\right) .
$$

In particular $\tilde{N}^{*}=\tilde{N}^{*}(E)$ is a subbundle under our regularity condition for $\left(R ; D^{1}, D^{2}\right)$.

3.2. $I G$ manifold $\left(W ; C^{*}, N\right)$ associated with $\left(R ; D^{1}, D^{2}, \tilde{N}(E)\right)$. Let $\left(R ; D^{1}, D^{2}\right)$ be a $P D$ manifold of second order, which is regular of type $\mathfrak{s}$. Moreover we assume that $R$ is regular with respect to $\mathrm{Ch}\left(D^{1}\right)$, i.e., the space $J=R / \mathrm{Ch}\left(D^{1}\right)$ of leaves of this foliation is a manifold of dimension $2 n+1$ such that each fibre of the projection $p: R \rightarrow J$ is connected and $p$ is a submersion. Then we have a differential system $C$ on $J$ of codimension 1 such that $D^{1}=p_{*}^{-1}(C)$ and $(J, C)$ becomes a contact manifold.

Now assume that there exists a $G_{0}(\mathfrak{s})$-invariant subspace $E$ of $V$ of dimension $r$. Then we have the first order covariant system $\tilde{N}=\tilde{N}(E)$ of $\left(R ; D^{1}, D^{2}\right)$. $\tilde{N}$ contains $D^{2}$ and is a subbundle of $D^{1}$ of codimension $r$. For a point $v \in R$, we see that $p_{*}\left(D^{1}(v)\right)=C(u), u=p(v)$ and $\iota(v)=p_{*}\left(D^{2}(v)\right)$ is a legendrian subspace of $(J, C)$, where $\iota: R \rightarrow L(J)$ is the canonical immersion (cf. $\S 4.1[27])$. Thus $w=p_{*}(\tilde{N}(v)) \subset C(u)$ is an involutive subspace of $(C(u), d \varpi)$ 
of codimension $r$ such that $w^{\perp} \subset \iota(v) \subset w$. Let $I^{r}(J)$ be the Involutive Grassmann bundle of $(J, C)$ of codimension $r$. Utilizing $\tilde{N}$, we will consider the map $\eta: R \rightarrow I^{r}(J)$ defined by

$$
\eta(v)=p_{*}(\tilde{N}(v)) \in I^{r}(J) \quad \text { for } \quad v \in R .
$$

By Realization Lemma for $(R, \tilde{N}, p, J)$, we have $\operatorname{Ker} \eta_{*}=\operatorname{Ch}\left(D^{1}\right) \cap \operatorname{Ch}(\tilde{N})$. Thus, if $\operatorname{Ch}\left(D^{1}\right) \cap$ $\mathrm{Ch}(\tilde{N})$ is a subbundle, $\eta$ is a map of constant rank so that the image $W=\operatorname{Im}(\eta)$ of $\eta$ is, at least locally, a submanifold of $I^{r}(J)$ such that $q: W \rightarrow J$ is a submersion, where $q=\left.\pi\right|_{W}$ and $\pi: I^{r}(J) \rightarrow J$ is the projection.

In the rest of this subsection, we assume that $\mathrm{Ch}\left(D^{1}\right) \cap \mathrm{Ch}(\tilde{N})$ is a subbundle so that $W=\operatorname{Im}(\eta)$ is a submanifold of $I^{r}(J)$ and will consider the relations of several covariant systems on $R$ and $W$. As a submanifold of $I^{r}(J)$ satisfying the condition (W.0), $W$ carries two differential systems $C^{*}$ and $N$ such that $C^{*}=q_{*}^{-1}(C)$. $\left(W ; C^{*}, N\right)$ is an IG manifold of corank $r$. $\left(W ; C^{*}, N\right)$ is called the IG manifold of corank $r$ associated with $\left(R ; D^{1}, D^{2}, \tilde{N}(E)\right)$. Then, by the definition of the canonical systems $\bar{C}, \bar{N}$ of $I^{r}(J)$ and the map $\eta$, we have

$$
\eta_{*}^{-1}\left(C^{*}\right)=D^{1} \quad \text { and } \quad \eta_{*}^{-1}(N)=\tilde{N} .
$$

Moreover, putting $\eta_{*}^{-1}(S(N)(w))=S(\tilde{N})(v) \subset \operatorname{Ch}\left(D^{1}\right)(v)$ for $w=\eta(v)$, we have

Lemma 3.1. (1) $\eta_{*}^{-1}\left(N^{\perp}\right)=\tilde{N}^{\perp}$.

(2) $\eta_{*}^{-1}\left(N^{*}\right)=\tilde{N}^{*}$.

(3) $\quad C h(\tilde{N})(v) \subset \tilde{N}^{\perp}(v) \subset D^{2}(v) \quad$ for $v \in R$.

(4) For a graded Lie algebra isomorphism $\phi$ of $\mathfrak{s}(v)$ onto $\mathfrak{s}$, $\phi(S(\tilde{N})(v))=\mathfrak{f} \cap\left(E^{\perp} \otimes_{S} V^{*}\right) \subset S^{2}\left(V^{*}\right)$.

Proof. (1) Put $\hat{N}=\eta_{*}^{-1}\left(N^{\perp}\right)$. For a point $v \in R$, from $p=q \cdot \eta$, we have $p_{*}(\hat{N}(v))=$ $q_{*}\left(N^{\perp}(w)\right)=w^{\perp} \subset \iota(v)$, where $w=\eta(v)$, which implies $\hat{N} \subset D^{2}$. From $\operatorname{Ker} q_{*}=\operatorname{Ch}\left(C^{*}\right) \subset N^{\perp}$ and $\operatorname{Ker} p_{*}=\operatorname{Ch}\left(D^{1}\right)=\eta_{*}^{-1}\left(\operatorname{Ch}\left(C^{*}\right)\right)$, we have $\operatorname{Ch}\left(D^{1}\right) \subset \hat{N} \subset D^{2}$. On the other hand, we have $\tilde{N}^{\perp}(v)=\left\{X \in D^{2}(v)=\mathfrak{s}_{-1}(v) \mid[X, \mathfrak{n}(E)]=0\right\}$ in terms of the symbol algebra $\mathfrak{s}(v)$. Moreover, from $\left[\Gamma\left(N^{\perp}\right), \Gamma(N)\right] \subset \Gamma\left(C^{*}\right)$, we have $[\Gamma(\hat{N}), \Gamma(\tilde{N})] \subset \Gamma\left(D^{1}\right)$. This implies $\hat{N} \subset \tilde{N}^{\perp}$. Then, comparing the ranks of both sides, we obtain $\hat{N}=\tilde{N}^{\perp}$.

(2) follows immediately from (1) and (3) follows from $\mathrm{Ch}(N)(w) \subset N^{\perp}(w)$ at each $w \in W$ (see $\S 2.3(1))$.

(4) From $\S 2.3(3)$, we have $S(N)(w)=\left\{X \in \operatorname{Ch}\left(C^{*}\right)(w) \mid\left[X, \Gamma\left(N^{\perp}\right)\right] \subset \Gamma(N)\right\}$. Thus, in terms of the symbol algebra, we get

$$
\phi(S(\tilde{N})(v))=\left\{f \in \mathfrak{f} \mid[f, E] \subset E^{\perp}\right\}=\{f \in \mathfrak{f} \mid[[f, E], E]=0\}=\mathfrak{f} \cap\left(E^{\perp} \otimes_{S} V^{*}\right) \subset S^{2}\left(V^{*}\right) .
$$

In particular, by Lemma $3.1(4), S(N)$ becomes a subbundle of $\mathrm{Ch}\left(C^{*}\right)$ under our regularity condition for $\left(R ; D^{1}, D^{2}, \tilde{N}(E)\right)$.

\section{Lagrange Grassmann Bundle $R(W)$ over an $I G$ manifold $\left(W ; C^{*}, N\right)$}

4.1. Lagrange Grassmann Bundle $R(W)$. Let $\left(W ; C^{*}, N\right)$ be an $I G$ manifold of corank $r$. We wil construct the Lagrange Grassmann bundle $R(W)$ over $\left(W ; C^{*}, N\right)$ and will examine the conditions when $R(W)$ becomes a $P D$ manifold of second order.

We assume that $W$ is regular with respect to $\mathrm{Ch}\left(C^{*}\right)$, i.e., the space $J=W / \mathrm{Ch}\left(C^{*}\right)$ of leaves of this foliation $\mathrm{Ch}\left(C^{*}\right)$ is a manifold of dimension $2 n+1$ such that $(J, C)$ is a contact manifold, where $C^{*}=q_{*}^{-1}(C)$ and $q: W \rightarrow J$ is a submersion. For a point $w \in W$, we 
will consider maximal isotropic subspaces of $\left(N(w), \gamma_{w}\right)$. Namely, we consider the Lagrange Grassmann bundle $R(W)$ over $\left(W ; C^{*}, N\right)$ :

$$
R(W)=\bigcup_{w \in W} R_{w}, \quad R_{w}=\left\{\hat{v} \subset N(w)\left|\gamma_{w}\right|_{\hat{v}}=0, \quad \hat{v} \text { is maximal }\right\} .
$$

$R(W)$ is a submanifold of the Grassmann bundle $J(W, n+t)$ over $W$, where $t=\operatorname{rank} \mathrm{Ch}\left(C^{*}\right)$. Moreover $v=q_{*}(\hat{v})$ is a legendrian subspace of $(J, C)$ such that $v \subset \iota(w)=q_{*}(N(w))$, where $\iota: W \rightarrow I^{r}(J)$ is the canonical immersion (see Theorem 2.1). Thus we define a map $\zeta$ : $R(W) \rightarrow L(J)$ by $\zeta(\hat{v})=q_{*}(\hat{v})$. Then we have

$$
\zeta\left(R_{w}\right)=\{v \in L(J) \mid v \subset \bar{w} \subset C(u)\} \cong L\left(\bar{w} / \bar{w}^{\perp}\right) \cong U(n-r) / O(n-r),
$$

where $u=q(w), \bar{w}=\iota(w)$ and $L\left(\bar{w} / \bar{w}^{\perp}\right)$ denotes the Lagrange Grassmann manifold of the symplectic vector space $\bar{w} / \bar{w}^{\perp}$ of dimension $2(n-r)$. Hence $R(W)$ is a manifold of dimension $k+\frac{1}{2}(n-r)(n-r+1)$, where $k=\operatorname{dim} W$ and $\zeta(R(W))$ is the collection of legendrian subspaces $v$ such that $v \subset \bar{w}$ for $\bar{w} \in \iota(W) \subset I^{r}(J)$.

Now we will describe the map $\zeta: R(W) \rightarrow L(J)$ in suitable coordinates. Let us fix a reference point $\hat{v}_{o} \in R(W)$ and put $w_{o}=\tau\left(\hat{v}_{o}\right)$, where $\tau: R(W) \rightarrow W$ is the projection. Moreover put $u_{o}=q\left(w_{o}\right) \in J . v_{o}=\zeta\left(\hat{v}_{o}\right)$ is a legendrian subspace of $\left(C\left(u_{o}\right), d \varpi\right)$ such that $v_{o} \subset \iota\left(w_{o}\right)=q_{*}\left(N\left(w_{o}\right)\right) \in I^{r}(J)$. Take a canonical coordinate system $\left(x_{1}, \ldots, x_{n}, z, p_{1}, \ldots, p_{n}\right)$ of $(J, C)$ defined on a neiborhood $U^{\prime}$ with origin $u_{o}$ such that

$$
\begin{gathered}
v_{o}=\left\{X \in T_{u_{o}}(J) \mid \varpi(X)=d p_{1}(X)=\cdots=d p_{n}(X)=0\right\} \subset \\
\iota\left(w_{o}\right)=\left\{X \in T_{u_{o}}(J) \mid \varpi(X)=d p_{1}(X)=\cdots=d p_{r}(X)=0\right\},
\end{gathered}
$$

where $\varpi=d z-\sum_{i=1}^{n} p_{i} d x_{i}$. Then we can introduce coordinate system $\left(x_{i}, x_{\alpha}, z, p_{i}, p_{\alpha}, a_{i}^{\alpha}, b_{i}^{\alpha}, s_{i j}\right)$ $(1 \leqq i \leqq j \leqq r, r+1 \leqq \alpha \leqq n)$ of $I^{r}(J)$ aroud $\iota\left(w_{o}\right)$ with origin $\iota\left(w_{o}\right)$ as in $\S 2.1$. Since $q: W \rightarrow J$ is a submersion, let us take a coordinate system $\left(x_{1}, \ldots, x_{n}, z, p_{1}, \ldots, p_{n}, \lambda_{1}, \ldots, \lambda_{t}\right)$ of $W$ on a neiborhood $U^{*} \subset q^{-1}\left(U^{\prime}\right)$ of $w_{o}$. Here $\left(x_{1}, \ldots, x_{n}, z, p_{1}, \ldots, p_{n}\right)$ are pullbacks to $U^{*}$ of coordinate functions on $U^{\prime}$. Then, on $U^{*}$, we have, from $\S 2.2$ and $\S 2.3, C^{*}=\{\varpi=0\}$,

$N=\left\{\varpi=\varpi_{1}^{*}=\cdots=\varpi_{r}^{*}=0\right\}, \quad N^{\perp}=\left\{\varpi=\varpi_{i}^{*}=\varpi_{\alpha}^{*}=\omega^{\alpha}=0 \quad(1 \leqq i \leqq r, r+1 \leqq \alpha \leqq n)\right\}$, where

$$
\begin{gathered}
\varpi_{i}^{*}=d p_{i}-\sum_{\alpha=r+1}^{n} a_{i}^{\alpha} d p_{\alpha}-\sum_{\alpha=r+1}^{n} b_{i}^{\alpha} d x_{\alpha}-\sum_{j=1}^{r}\left(s_{i j}-\sum_{\alpha=r+1}^{n} a_{i}^{\alpha} b_{j}^{\alpha}\right) d x_{j} \quad(1 \leqq i \leqq r), \\
\varpi_{\alpha}^{*}=d p_{\alpha}-\sum_{j=1}^{r} b_{j}^{\alpha} d x_{j} \quad \text { and } \quad \omega^{\alpha}=d x_{\alpha}+\sum_{i=1}^{r} a_{i}^{\alpha} d x_{i} \quad(r+1 \leqq \alpha \leqq n) .
\end{gathered}
$$

Here we note that $a_{i}^{\alpha}=\iota^{*}\left(a_{i}^{\alpha}\right), b_{i}^{\alpha}=\iota^{*}\left(b_{i}^{\alpha}\right)$ and $s_{i j}=\iota^{*}\left(s_{i j}\right)$ are functions of $\left(x_{1}, \ldots, x_{n}, z\right.$, $\left.p_{1}, \ldots, p_{n}, \lambda_{1}, \ldots, \lambda_{t}\right)$ on $U^{*}$. Moreover we have, from $\S 2.2$ and $\S 2.3$,

$$
\mathrm{Ch}\left(C^{*}\right)=\left\{\varpi=\varpi_{i}^{*}=\varpi_{\alpha}^{*}=\omega^{\alpha}=d x_{i}=0 \quad(1 \leqq i \leqq r, r+1 \leqq \alpha \leqq n)\right\},
$$

and

$$
S(N)(w)=\left\{\varpi=\varpi_{i}^{*}=\varpi_{\alpha}^{*}=\omega^{\alpha}=d x_{i}=\varpi_{i j}=0 \quad(1 \leqq i \leqq j \leqq r, r+1 \leqq \alpha \leqq n)\right\} .
$$

where $\varpi_{i j}=d s_{i j}-\sum_{\alpha=r+1}^{n}\left(a_{i}^{\alpha} d b_{j}^{\alpha}+a_{j}^{\alpha} d b_{i}^{\alpha}\right)$.

On $U^{*}, 1$-forms $\left\{\varpi, \varpi_{i}^{*}, d p_{\alpha}, d x_{i}, d x_{\alpha}, d \lambda_{a} \quad(1 \leqq i \leqq r, r+1 \leqq \alpha \leqq n, 1 \leqq a \leqq t)\right\}$ form a coframe. By our choice of the coordinate system, we have

$$
\hat{v}_{o}=q_{*}^{-1}\left(v_{o}\right)=\left\{X \in T_{w_{o}}(W) \mid \varpi(X)=\varpi_{i}^{*}(X)=d p_{\alpha}(X)=0 \quad(1 \leqq i \leqq r, r+1 \leqq \alpha \leqq n)\right\} .
$$


Hence we will work on the following neiborhood $\hat{U}$ of $\hat{v}_{o}$ in $J(W, n+t)$ :

$$
\hat{U}=\left\{\hat{v} \in \hat{\tau}^{-1}\left(U^{*}\right) \mid d x_{1}, \ldots, d x_{n}, d \lambda_{1}, \ldots, d \lambda_{t} \text { are linearly independent on } \hat{v}\right\},
$$

where $\hat{\tau}: J(W, n+t) \rightarrow W$ is the projection. For a point $\hat{v} \in R(W) \cap \hat{U}$, from $\hat{v} \subset N(w)$, we have

$$
\left.\varpi\right|_{\hat{v}}=\left.\varpi_{i}^{*}\right|_{\hat{v}}=0 \quad(i=1, \ldots, r) .
$$

Thus, by expressing $\left.d p_{\alpha}\right|_{\hat{v}}$ as a linear combination of $\left.d x_{i}\right|_{\hat{v}},\left.d x_{\alpha}\right|_{\hat{v}}$ and $\left.d \lambda_{a}\right|_{\hat{v}}$, we see that $\hat{v}$ is defined by

where

$$
\hat{v}=\left\{X \in T_{w}(W) \mid \varpi(X)=\varpi_{i}^{*}(X)=\pi_{\alpha}^{*}(\hat{v})(X)=0\right\},
$$

$$
\pi_{\alpha}^{*}(\hat{v})=d p_{\alpha}-\sum_{i=1}^{r} p_{\alpha i}^{*}(\hat{v}) d x_{i}-\sum_{\beta=r+1}^{n} p_{\alpha \beta}^{*}(\hat{v}) d x_{\beta}-\sum_{a=1}^{t} p_{\alpha}^{a}(\hat{v}) d \lambda_{a} .
$$

Here $\left(x_{i}, x_{\alpha}, z, p_{i}, p_{\alpha}, \lambda_{a}, p_{\alpha i}^{*}, p_{\alpha \beta}^{*}, p_{\alpha}^{a}\right)(1 \leqq i \leqq r, r+1 \leqq \alpha, \beta \leqq n, 1 \leqq a \leqq t)$ constitute a coordinate system on $\hat{U}$ of the submanifold $J(W, n+t ; N)=\{\hat{v} \in J(W, n+t) \mid \hat{v} \subset N(w)\}$ of $J(W, n+t)$. On this coordinate system, $\left.\gamma_{w}\right|_{\hat{v}}=0$ is equivalent to $\left.d \varpi\right|_{\hat{v}}=0$, i.e.,

$$
d \varpi \equiv 0 \quad\left(\bmod \quad \varpi, \varpi_{1}^{*}, \ldots, \varpi_{r}^{*}, \pi_{r+1}^{*}(\hat{v}), \ldots, \pi_{n}^{*}(\hat{v})\right) .
$$

Hence, from $\pi_{\alpha}^{*}=\varpi_{\alpha}^{*}-\sum_{\beta=r+1}^{n} p_{\alpha \beta}^{*} \omega^{\beta}-\sum_{i=1}^{r}\left(p_{\alpha i}^{*}-b_{i}^{\alpha}-\sum_{\beta=r+1}^{n} p_{\alpha \beta}^{*} a_{i}^{\beta}\right) d x_{i}-\sum_{a=1}^{t} p_{\alpha}^{a} d \lambda_{a}$, we calculate

$$
\begin{aligned}
& d \varpi \equiv \sum_{\alpha=r+1}^{n} \omega^{\alpha} \wedge \varpi_{\alpha}^{*} \\
& \equiv \sum_{\alpha=r+1}^{n}\left\{\sum_{\beta=r+1}^{n} p_{\alpha \beta}^{*} \omega^{\alpha} \wedge \omega^{\beta}+\sum_{i=1}^{r}\left(p_{\alpha i}^{*}-b_{i}^{\alpha}-\sum_{\beta=r+1}^{n} p_{\alpha \beta}^{*} a_{i}^{\beta}\right) \omega^{\alpha} \wedge d x_{i}+\sum_{a=1}^{t} p_{\alpha}^{a} \omega^{\alpha} \wedge d \lambda_{a}\right\} \\
&\left(\bmod \quad \varpi, \varpi_{1}^{*}, \ldots, \varpi_{r}^{*}, \pi_{r+1}^{*}, \ldots, \pi_{n}^{*}\right) .
\end{aligned}
$$

Thus we see that $R(W)$ is defined in this coordinate system by

$$
p_{\alpha \beta}^{*}=p_{\beta \alpha}^{*}, \quad p_{\alpha i}^{*}=b_{i}^{\alpha}+\sum_{\beta=r+1}^{n} p_{\alpha \beta}^{*} a_{i}^{\beta}, \quad p_{\alpha}^{a}=0 \quad(r+1 \leqq \alpha, \beta \leqq n, 1 \leqq i \leqq r, 1 \leqq a \leqq t) .
$$

In this way, we obtain a coordinate system $\left(x_{1}, \ldots, x_{n}, z, p_{1}, \ldots, p_{n}, \lambda_{1}, \ldots, \lambda_{t}, p_{\alpha \beta}^{*}\right)(r+1 \leqq$ $\alpha \leqq \beta \leqq n)$ of $R(W)$ on $\hat{U} \cap R(W)$. On the other hand, we have a canonical coordinate system $\left(x_{1}, \ldots, x_{n}, z, p_{1}, \ldots, p_{n}, p_{k l}\right)(1 \leqq k \leqq l \leqq n), p_{k l}=p_{l k}$, of $(L(J), E)$, which is subordinate to $U^{\prime} ;\left(x_{1}, \ldots, x_{n}, z, p_{1}, \ldots, p_{n}\right)$, defined on a neiborhood $U$ with origin $v_{o}=\zeta\left(\hat{v}_{o}\right)$ such that

$$
E=\left\{\varpi=\varpi_{1}=\cdots=\varpi_{n}=0\right\},
$$

where $\varpi_{k}=d p_{k}-\sum_{l=1}^{n} p_{k l} d x_{l}(1 \leqq k \leqq n), U=\left\{v \in \pi^{-1}\left(U^{\prime}\right)\left|d x_{1} \wedge \cdots \wedge d x_{n}\right|_{v} \neq 0\right\}$ and $\pi: L(J) \rightarrow J$ is the projection. By the definition of the canonical system $E$ on $L(J)$, we have $E(v)=\pi_{*}^{-1}(v)$. Hence, from $\pi \cdot \zeta=q \cdot \tau$ and $v=q_{*}(\hat{v})$, we have

$$
\zeta_{*}^{-1}(E(v))=(\pi \cdot \zeta)_{*}^{-1}(v)=(q \cdot \tau)_{*}^{-1}(v)=\tau_{*}^{-1}(\hat{v}) .
$$

Thus, on $R(W) \cap \hat{U}$, we obtain

$$
\left\{\zeta^{*} \varpi=\zeta^{*} \varpi_{1}=\cdots=\zeta^{*} \varpi_{n}=0\right\}=\left\{\varpi=\varpi_{1}^{*}=\cdots=\varpi_{r}^{*}=\pi_{r+1}^{*}=\cdots=\pi_{n}^{*}=0\right\} .
$$

We have

$$
\zeta^{*} \varpi=\varpi, \quad \zeta^{*} \varpi_{k}=d p_{k}-\sum_{l=1}^{n} \zeta^{*} p_{k l} d x_{l} \quad(1 \leqq k \leqq n)
$$


and

$$
\pi_{\alpha}^{*}=\varpi_{\alpha}^{*}-\sum_{\beta=r+1}^{n} p_{\alpha \beta}^{*} \omega^{\beta}=d p_{\alpha}-\sum_{i=1}^{r} p_{\alpha i}^{*} d x_{i}-\sum_{\beta=r+1}^{n} p_{\alpha \beta}^{*} d x_{\beta} \quad(r+1 \leqq \alpha \leqq n) .
$$

Moreover we calculate

$$
\begin{aligned}
\varpi_{i}^{*} & =d p_{i}-\sum_{\alpha=r+1}^{n} a_{i}^{\alpha} d p_{\alpha}-\sum_{\alpha=r+1}^{n} b_{i}^{\alpha} d x_{\alpha}-\sum_{j=1}^{r}\left(s_{i j}-\sum_{\alpha=r+1}^{n} a_{i}^{\alpha} b_{j}^{\alpha}\right) d x_{j} \\
& \equiv d p_{i}-\sum_{\alpha=r+1}^{n} a_{i}^{\alpha}\left(\sum_{j=1}^{r} p_{\alpha j}^{*} d x_{j}+\sum_{\beta=r+1}^{n} p_{\alpha \beta}^{*} d x_{\beta}\right)-\sum_{\alpha=r+1}^{n} b_{i}^{\alpha} d x_{\alpha}-\sum_{j=1}^{r}\left(s_{i j}-\sum_{\alpha=r+1}^{n} a_{i}^{\alpha} b_{j}^{\alpha}\right) d x_{j} \\
& \equiv d p_{i}-\sum_{j=1}^{r} p_{i j}^{*} d x_{j}-\sum_{\alpha=r+1}^{n} p_{\alpha i}^{*} d x_{\alpha} \quad\left(\bmod \quad \pi_{r+1}^{*}, \ldots, \pi_{n}^{*}\right) .
\end{aligned}
$$

where $p_{i j}^{*}=s_{i j}+\sum_{\alpha, \beta=r+1}^{n} p_{\alpha \beta}^{*} a_{i}^{\alpha} a_{j}^{\beta}$. Hence we obtain

$$
\zeta^{*} p_{\alpha \beta}=p_{\alpha \beta}^{*}, \quad \zeta^{*} p_{\alpha i}=b_{i}^{\alpha}+\sum_{\beta=r+1}^{n} p_{\alpha \beta}^{*} a_{i}^{\beta}, \quad \zeta^{*} p_{i j}=s_{i j}+\sum_{\alpha=r+1}^{n} \sum_{\beta=r+1}^{n} p_{\alpha \beta}^{*} a_{i}^{\alpha} a_{j}^{\beta},
$$

which describe the map $\zeta: R(W) \rightarrow L(J)$ in the above coordinate systems.

4.2. Differential Systems on $R(W)$. We have several differential systems naturally defined on $R(W)$. First, $D_{W}^{1}=\tau_{*}^{-1}\left(C^{*}\right), N_{W}=\tau_{*}^{-1}(N)$ and $N_{W}^{\perp}=\tau_{*}^{-1}\left(N^{\perp}\right)$ are the lifts of $C^{*}, N$ and $N^{\perp}$ respectively and the canonical system $D_{W}^{2}$ is defined by

$$
D_{W}^{2}(\hat{v})=\tau_{*}^{-1}(\hat{v}) \subset T_{\hat{v}}(R(W)) \stackrel{\tau_{*}}{\longrightarrow} T_{w}(W) \quad \text { at each } \quad \hat{v} \in R(W) .
$$

Then, on $R(W) \cap \hat{U}$, we have

$$
\begin{aligned}
& D_{W}^{1}=\{\varpi=0\}, \quad \operatorname{Ch}\left(D_{W}^{1}\right)=\left\{\varpi=\varpi_{i}^{*}=\varpi_{\alpha}^{*}=\omega^{\alpha}=d x_{i}=0 \quad(1 \leqq i \leqq r, r+1 \leqq \alpha \leqq n)\right\}, \\
& N_{W}=\left\{\varpi=\varpi_{i}^{*}=0(1 \leqq i \leqq r)\right\}, \quad N_{W}^{\perp}=\left\{\varpi=\varpi_{i}^{*}=\varpi_{\alpha}^{*}=\omega^{\alpha}=0(1 \leqq i \leqq r, r+1 \leqq \alpha \leqq n)\right\}, \\
& D_{W}^{2}=\left\{\varpi=\varpi_{i}^{*}=\varpi_{\alpha}^{*}-\sum_{\beta=r+1}^{n} p_{\alpha \beta}^{*} \omega^{\beta}=0 \quad(1 \leqq i \leqq r, r+1 \leqq \alpha \leqq n)\right\} .
\end{aligned}
$$

For these systems, we have

Lemma 4.1. (1) $\zeta_{*}^{-1}(E)=D_{W}^{2}$ and $\zeta_{*}^{-1}(\partial E)=D_{W}^{1}$.

(2) $D_{W}^{1}$ and $D_{W}^{2}$ are differential systems of codimension 1 and $n+1$ respectively.

(3) $\partial D_{W}^{2} \subset D_{W}^{1}$

(4) $C h\left(D_{W}^{1}\right)$ is a subbundle of $D_{W}^{2}$ of codimension $n$.

(5) $\quad N_{W}$ is a subbundle of $D_{W}^{1}$ of codimension $r$, which contains $D_{W}^{2}$ such that $C h\left(N_{W}\right)(\hat{v}) \subset D_{W}^{2}(\hat{v})$ at each $\hat{v} \in R(W)$.

(6) $\quad C h\left(D_{W}^{1}\right) \cap C h\left(N_{W}\right)=\operatorname{Ker} \tau_{*}$.

(7) $\quad C h\left(D_{W}^{1}\right)(\hat{v}) \cap C h\left(D_{W}^{2}\right)(\hat{v})=\operatorname{Ker} \zeta_{*}(\hat{v})$ at each $\hat{v} \in R(W)$.

(8) $\operatorname{Ker} \tau_{*} \cap \operatorname{Ker} \zeta_{*}=\{0\}$.

Proof. (1) follows from (4.2) and $\zeta^{*} \varpi=\varpi$. (2) and (4) are obvious from the above and (3) follows from $d \varpi \equiv 0\left(\bmod \varpi, \varpi_{1}^{*}, \ldots, \varpi_{r}^{*}, \pi_{r+1}^{*}, \ldots, \pi_{n}^{*}\right)$, where $\pi_{\alpha}^{*}=\varpi_{\alpha}^{*}-\sum_{\beta=r+1}^{n} p_{\alpha \beta}^{*} \omega^{\beta}$ $(r+1 \leqq \alpha \leqq n)$. The first half of (5) is obvious from the above and $\operatorname{Ch}\left(N_{W}\right)(\hat{v}) \subset D_{W}^{2}(\hat{v})$ follows from $N_{W}^{\perp} \subset D_{W}^{2}$ and $\mathrm{Ch}(N)(w) \subset N^{\perp}(w)$, where $w=\tau(\hat{v})$. (6) follows from $\mathrm{Ch}\left(C^{*}\right) \cap$ $\operatorname{Ch}(N)=\{0\}$. By $\operatorname{Ker}(q \cdot \tau)_{*}=\tau_{*}^{-1}\left(\operatorname{Ch}\left(C^{*}\right)\right)=\operatorname{Ch}\left(D_{W}^{1}\right)$ and $\zeta(\hat{v})=q_{*}(\hat{v})=q_{*}\left(\tau_{*}\left(D_{W}^{2}(\hat{v})\right)\right)$, 
(7) follows from the Realization Lemma for $\left(R(W), D_{W}^{2}, q \cdot \tau, J\right)$. Finally (8) follows from $\operatorname{Ker} \zeta_{*} \subset\left\{d p_{\alpha \beta}^{*}=0(r+1 \leqq \alpha \leqq \beta \leqq n)\right\}$ and

$$
\operatorname{Ker} \tau_{*}=\left\{d z=d p_{1}=\cdots=d p_{n}=d x_{1}=\cdots=d x_{n}=d \lambda_{1}=\cdots=d \lambda_{t}=0\right\} .
$$

Thus, by (2),(3),(4) and (7) of Lemma 4.1, we see that $\left(R(W) ; D_{W}^{1}, D_{W}^{2}\right)$ is a $P D$ manifold of second order iff $\zeta: R(W) \rightarrow L(J)$ is an immersion. We will describe this condition in terms of invariants of $\left(W ; C^{*}, N\right)$. For a point $\hat{v} \in R(W)$, put

$$
A(\hat{v})=\tau_{*}\left(\operatorname{Ch}\left(D_{W}^{1}\right)(\hat{v}) \cap \operatorname{Ch}\left(D_{W}^{2}\right)(\hat{v})\right) \subset \operatorname{Ch}\left(C^{*}\right)(w)=\tau_{*}\left(\operatorname{Ch}\left(D_{W}^{1}\right)(\hat{v})\right), \quad w=\tau(\hat{v}) .
$$

By Lemma $4.1(8), A(\hat{v})=\{0\}$ iff $\operatorname{Ker} \zeta_{*}(\hat{v})=\{0\}$, i.e., $\zeta$ is an immersion around $\hat{v}$. On the neiborhood $R(W) \cap \hat{U}$, we have

$$
\begin{aligned}
d \pi_{\alpha}^{*} & =d \varpi_{\alpha}^{*}-\sum_{\beta=r+1}^{n} p_{\alpha \beta}^{*} d \omega^{\beta}+\sum_{\beta=r+1}^{n} \omega^{\beta} \wedge d p_{\alpha \beta}^{*} \\
& =\sum_{i=1}^{r} d x_{i} \wedge\left(d b_{i}^{\alpha}+\sum_{\beta=r+1}^{n} p_{\alpha \beta}^{*} d a_{i}^{\beta}\right)+\sum_{\beta=r+1}^{n} \omega^{\beta} \wedge d p_{\alpha \beta}^{*} .
\end{aligned}
$$

Hence, from (2.1), we get

$$
\left\{\begin{aligned}
& d \varpi \equiv 0 \\
& d \varpi_{i}^{*} \equiv \sum_{j=1}^{r} d x_{j} \wedge \varpi_{i j}+\sum_{\alpha=r+1}^{n} \omega^{\alpha} \wedge \pi_{\alpha i} \quad\left(\bmod \varpi, \varpi_{1}^{*}, \ldots, \varpi_{r}^{*}, \pi_{r+1}^{*}, \ldots, \pi_{n}^{*}\right) \\
& d \pi_{\alpha} \equiv \sum_{i=1}^{r} d x_{i} \wedge \pi_{\alpha i}+\sum_{\beta=r+1}^{n} \omega^{\beta} \wedge d p_{\alpha \beta}^{*} .
\end{aligned}\right.
$$

for $1 \leqq i \leqq r, r+1 \leqq \alpha \leqq n$, where $\pi_{\alpha i}=d b_{i}^{\alpha}+\sum_{\beta=r+1}^{n} p_{\alpha \beta}^{*} d a_{i}^{\beta}$. From

$$
\operatorname{Ch}\left(D_{W}^{1}\right)=\left\{\varpi=\varpi_{i}^{*}=\varpi_{\alpha}^{*}=\omega^{\alpha}=d x_{i}=0 \quad(1 \leqq i \leqq r, r+1 \leqq \alpha \leqq n)\right\},
$$

we obtain

$$
\begin{aligned}
& \operatorname{Ch}\left(D_{W}^{1}\right)(\hat{v}) \cap \operatorname{Ch}\left(D_{W}^{2}\right)(\hat{v})= \\
& \left\{X \in \operatorname{Ch}\left(D_{W}^{1}\right)(\hat{v}) \mid \varpi_{i j}(X)=\pi_{\alpha i}(X)=d p_{\alpha \beta}^{*}(X)=0(1 \leqq i \leqq j \leqq r, r+1 \leqq \alpha \leqq \beta \leqq n)\right\} .
\end{aligned}
$$

Thus, by (4.1), we get

$$
A(\hat{v})=\left\{X \in S(N)(w) \mid\left(d b_{i}^{\alpha}+\sum_{\beta=r+1}^{n} p_{\alpha \beta}^{*} d a_{i}^{\beta}\right)(X)=0(1 \leqq i \leqq r, r+1 \leqq \alpha \leqq n)\right\},
$$

on $R(W) \cap \hat{U}$ and $\hat{v}=\left\{\varpi=\varpi_{i}^{*}=\varpi_{\alpha}^{*}-\sum_{\beta=r+1}^{n} p_{\alpha \beta}^{*} \omega^{\beta}=0(1 \leqq i \leqq r, r+1 \leqq \alpha \leqq n)\right\}$. We will describe this subspace of $S(N)(w)$ in terms of invariants of $\left(W ; C^{*}, N\right)$.

For a point $\hat{v} \in R(W), \hat{v}$ is a maximal isotropic subspace in $N(w), w=\tau(\hat{v})$, which contains $N^{\perp}(w)$. We put $E^{\perp}(\hat{v})=N(w) / \hat{v}\left(\cong N_{W}(\hat{v}) / D_{W}^{2}(\hat{v})\right)$. Then we get the projection $\pi_{\hat{v}}: \mathfrak{t}_{N}(w)=$ $N(w) / N^{\perp}(w) \rightarrow E^{\perp}(\hat{v})=N(w) / \hat{v}$, which induces, from (2.3), the following map:

$$
\gamma_{c}(\hat{v}): S(N)(w) \rightarrow E^{\perp}(\hat{v}) \otimes E(w)^{*}
$$

by $\gamma_{c}(\hat{v})=\left(\pi_{\hat{v}} \otimes i d_{E(w)^{*}}\right) \cdot \gamma_{c}$. We can describe $A(\hat{v})$ as follows; 
Lemma 4.2. (1) $A(\hat{v})=\operatorname{Ker} \gamma_{c}(\hat{v})$

(2) $\left.\left.A(\hat{v})=\{X \in S(N)(w) \mid X\rfloor d \varpi \equiv X\rfloor d \varpi_{1}^{*} \equiv \cdots \equiv X\right\rfloor d \varpi_{r}^{*} \equiv 0 \quad\left(\bmod \quad(\hat{v})^{\perp}\right)\right\}$, where $N=\left\{\varpi=\varpi_{1}^{*}=\cdots=\varpi_{r}^{*}=0\right\}$.

Proof. By (4.4), for a vector $X \in S(N)(w)$, we have

$$
\left\{\begin{array}{l}
X\rfloor d \varpi \equiv 0, \quad X\rfloor d \varpi_{i}^{*} \equiv 0 \quad\left(\bmod \varpi, \varpi_{i}^{*}, \varpi_{\alpha}^{*}, \omega^{\alpha}(1 \leqq r \leqq r, r+1 \leqq \alpha \leqq n)\right) \\
X\rfloor d \pi_{\alpha}^{*} \equiv-\sum_{i=1}^{r}\left(d b_{i}^{\alpha}+\sum_{\beta=r+1}^{n} p_{\alpha \beta}^{*} d a_{i}^{\beta}\right)(X) d x_{i} .
\end{array}\right.
$$

Hence we have

$$
A(\hat{v})=\left\{X \in S(N)(w) \mid\left[X, \Gamma\left(N^{\perp}\right)\right] \subset \hat{v}\right\} .
$$

On the other hand, for $X \in S(N)(w)$ and $Y \in N^{\perp}(w)$, we take $\tilde{X} \in \Gamma(S(N))$ and $\tilde{Y} \in \Gamma\left(N^{\perp}\right)$ such that $X=\tilde{X}_{w}$ and $Y=\tilde{Y}_{w}$. Then, from $(2.3), \gamma_{c}: S(N)(w) \rightarrow \mathfrak{t}_{N}(w) \otimes E^{*}(w)$ is defined by $\hat{\gamma}_{c}(X, Y)=\pi_{N}\left([\tilde{X}, \tilde{Y}]_{w}\right) \in \mathfrak{t}_{N}(w)=N(w) / N^{\perp}(w)$. Thus $\gamma_{c}(\hat{v})$ is defined by

$$
\hat{\gamma}_{c}(\hat{v})(X, Y)=\hat{\pi}_{\hat{v}}\left([\tilde{X}, \tilde{Y}]_{w}\right) \in E^{\perp}(\hat{v})=N(w) / \hat{v}
$$

where $\hat{\pi}_{\hat{v}}: N(w) \rightarrow E^{\perp}(\hat{v})$ is the projection. Hence $[\tilde{X}, \tilde{Y}]_{w} \in \hat{v}$ for $\forall Y \in N^{\perp}(w)$ iff $\hat{\gamma}_{c}(\hat{v})(X, Y)=0$ for $\forall Y \in N^{\perp}(w)$, i.e., $\gamma_{c}(\hat{v})(X)=0$, which implies $A(\hat{v})=\operatorname{Ker} \gamma_{c}(\hat{v})$.

Moreover, by (4.4), for a vector $X \in S(N)(w)$, we have

$$
\left\{\begin{array}{l}
X\rfloor d \varpi \equiv 0 \quad\left(\bmod \varpi, \varpi_{1}^{*}, \ldots, \varpi_{r}^{*}, \pi_{r+1}^{*}, \ldots, \pi_{n}^{*}\right) \\
X\rfloor d \varpi_{i}^{*} \equiv-\sum_{\alpha=r+1}^{n} \pi_{\alpha i}(X) \omega^{\alpha} \quad(1 \leqq i \leqq r)
\end{array}\right.
$$

Here $\pi_{\alpha}^{*}=\varpi_{\alpha}^{*}-\sum_{\beta=r+1}^{n} p_{\alpha \beta}^{*} \omega^{\beta}$ and $\pi_{\alpha i}=d b_{i}^{\alpha}+\sum_{\beta=r+1}^{n} p_{\alpha \beta}^{*} d a_{i}^{\beta}(1 \leqq i \leqq r, r+1 \leqq \alpha \leqq n)$. Hence, from (4.5), we get

$$
\begin{aligned}
A(\hat{v}) & \left.\left.=\{X \in S(N)(w) \mid X\rfloor d \varpi \equiv X\rfloor d \varpi_{1}^{*} \equiv \cdots \equiv X\right\rfloor d \varpi_{r}^{*} \equiv 0 \quad\left(\bmod \quad(\hat{v})^{\perp}\right)\right\} \\
& =\{X \in S(N)(w) \mid[X, Y] \in N(w) \text { for } \forall Y \in \hat{v}\}
\end{aligned}
$$

Finally we will indicate the relation between the symbol algebra $\mathfrak{s}(\hat{v})$ of $\left(R(W) ; D_{W}^{1}, D_{W}^{2}\right)$ at $\hat{v} \in R(W)$ and the invariants of $\left(W ; C^{*}, N\right)$ at $w=\tau(\hat{v})$, when $\operatorname{Ker} \zeta_{*}$ is trivial. In the rest of this subsection, we assume that $\zeta: R(W) \rightarrow L(J)$ is an immersion. Hence $\left(R(W) ; D_{W}^{1}, D_{W}^{2}\right)$ is a $P D$ manifold of second order. Moreover we assume that $R(W)$ satisfies the following condition $(C)$ :

$$
\text { (C) } \quad \hat{p}^{(1)}: R(W)^{(1)} \rightarrow R(W) \text { is onto. }
$$

where $R(W)^{(1)}$ is the first prolongation of $\left(R(W) ; D_{W}^{1}, D_{W}^{2}\right)$, i.e., there exists an $n$-dimensional integral element $V=V(\hat{v})$ of $\left(R(W), D_{W}^{2}\right)$ at each $\hat{v} \in R(W)$ such that $D_{W}^{2}(\hat{v})=V \oplus \mathfrak{f}(\hat{v})$, where $\mathfrak{f}(\hat{v})=\operatorname{Ker}(q \cdot \tau)_{*}(\hat{v})=\operatorname{Ch}\left(D_{W}^{1}\right)(\hat{v})=\tau_{*}^{-1}\left(\operatorname{Ch}\left(C^{*}\right)(w)\right)$ and $w=\tau(\hat{v})$.

Let $\mathfrak{s}(\hat{v})=\mathfrak{s}_{-3}(\hat{v}) \oplus \mathfrak{s}_{-2}(\hat{v}) \oplus \mathfrak{s}_{-1}(\hat{v})$ be the symbol algebra of $\left(R(W) ; D_{W}^{1}, D_{W}^{2}\right)$ at $\hat{v} \in R(W)$, where $\mathfrak{s}_{-3}(\hat{v})=T_{\hat{v}}(R(W)) / D_{W}^{1}(\hat{v}), \mathfrak{s}_{-2}(\hat{v})=D_{W}^{1}(\hat{v}) / D_{W}^{2}(\hat{v})$ and $\mathfrak{s}_{-1}(\hat{v})=D_{W}^{2}(\hat{v})$. Fixing a basis of $\mathfrak{s}_{-3}(\hat{v})$, we have (see $\S 3.1[27]$ )

$$
\mathfrak{s}_{-3}(\hat{v}) \cong \mathbb{R}, \quad \mathfrak{s}_{-2}(\hat{v}) \cong V^{*}, \quad \mathfrak{s}_{-1}(\hat{v})=V \oplus \mathfrak{f}(\hat{v}) \quad \text { and } \quad \mathfrak{f}(\hat{v}) \subset S^{2}\left(V^{*}\right)
$$

From $N_{W}^{\perp}(\hat{v}) \supset \mathrm{Ch}\left(D_{W}^{1}\right)(\hat{v})=\mathfrak{f}(\hat{v})$, we obtain the subspace $E(\hat{v})$ of $V(\hat{v})$ of dimension $r$ by $E(\hat{v})=V(\hat{v}) \cap N_{W}^{\perp}(\hat{v})$. Then $N_{W}(\hat{v}) / D_{W}^{2}(\hat{v}) \subset \mathfrak{s}_{-2}(\hat{v})=D_{W}^{1}(\hat{v}) / D_{W}^{2}(\hat{v})$ corresponds to 
$E(\hat{v})^{\perp} \subset V(\hat{v})^{*}$ in the identification $\mathfrak{s}_{-2}(\hat{v}) \cong V(\hat{v})^{*}$. Moreover $\tau_{*}: T_{\hat{v}}(R(W)) \rightarrow T_{w}(W)$ induces the identifications:

$$
\mathfrak{s}_{-3}(\hat{v}) \cong \mathfrak{t}_{-3}(w) \cong \mathbb{R}, \quad E(\hat{v}) \cong E(w)=N^{\perp}(w) / \mathrm{Ch}\left(C^{*}\right)(w), \quad N_{W}(\hat{v}) / D_{W}^{2}(\hat{v}) \cong N(w) / \hat{v} .
$$

In particular, $\tau_{*}: \mathfrak{f}(\hat{v}) \rightarrow \mathrm{Ch}\left(C^{*}\right)(w)$ induces the identification:

$$
\mathfrak{f}(\hat{v}) / S\left(N_{W}\right)(\hat{v}) \cong \mathrm{Ch}\left(C^{*}\right)(w) / S(N)(w),
$$

where $S\left(N_{W}\right)(\hat{v})=\tau_{*}^{-1}(S(N)(w))$. By Lemma $3.1(4)$, we have

$$
S\left(N_{W}\right)(\hat{v})=\mathfrak{f}(\hat{v}) \cap\left(E(\hat{v})^{\perp} \otimes_{S} V(\hat{v})^{*}\right) \subset S^{2}\left(V(\hat{v})^{*}\right) .
$$

Let us take a complimentary subspace $H$ of $V$ such that $V=E \oplus H$. Then $V^{*}=E^{\perp} \oplus H^{\perp}$ and $H^{\perp}$ is naturally identified with $V^{*} / E^{\perp}$, hence with $E^{*}$. Then we have

$$
S^{2}\left(V^{*}\right)=E^{\perp} \otimes_{S} V^{*} \oplus S^{2}\left(H^{\perp}\right) \quad \text { and } \quad E^{\perp} \otimes_{S} V^{*}=S^{2}\left(E^{\perp}\right) \oplus E^{\perp} \otimes_{S} H^{\perp} .
$$

Now the inclusion map $\iota: E \rightarrow V$ induces $\iota^{*}: V^{*} \rightarrow E^{*}$ and $\hat{\iota}: S^{2}\left(V^{*}\right) \rightarrow S^{2}\left(E^{*}\right)$ such that $\operatorname{Ker} \iota^{*}=E^{\perp}$ and Ker $\hat{\iota}=E^{\perp} \otimes_{S} V^{*}$.

Furthermore, under the identification: $\mathfrak{s}_{-3}(\hat{v}) \cong \mathfrak{t}_{-3}(w) \cong \mathbb{R}$ by fixing a basis of $\mathfrak{s}_{-3}(\hat{v})$, we have the following commutative diagram:

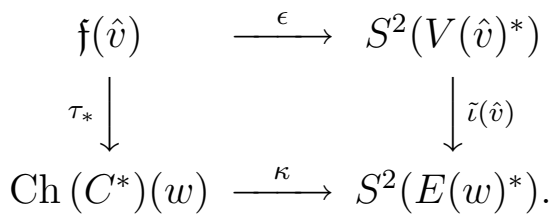

where $\epsilon: \mathfrak{f}(\hat{v}) \rightarrow S^{2}\left(V(\hat{v})^{*}\right)$ is given by $\epsilon(f)\left(v_{1}, v_{2}\right)=\left[\left[f, v_{1}\right], v_{2}\right]$ in $\mathfrak{s}(\hat{v})$ for $f \in \mathfrak{f}(\hat{v})$ and $v_{1}, v_{2} \in V(\hat{v}), \tilde{\iota}(\hat{v})=\left(\hat{\tau}^{*}\right)^{-1} \cdot \hat{\iota}(\hat{v}), \hat{\imath}(\hat{v}): S^{2}\left(V(\hat{v})^{*}\right) \rightarrow S^{2}\left(E(\hat{v})^{*}\right)$ and $\hat{\tau}^{*}: S^{2}\left(E(w)^{*}\right) \rightarrow$ $S^{2}\left(E(\hat{v})^{*}\right)$ is induced by the linear isomorphism $\hat{\tau}: E(\hat{v}) \rightarrow E(w)$. Hence $\kappa\left(\mathrm{Ch}\left(C^{*}\right)(w)\right)=$ $\hat{\kappa}\left(\mathrm{Ch}\left(C^{*}\right)(w) / S(N)(w)\right) \subset S^{2}\left(E(w)^{*}\right)$ is sent, by $\hat{\tau}^{*}$, to the image $\hat{\imath}(\hat{v})(\mathfrak{f}(\hat{v})) \subset S^{2}\left(E(\hat{v})^{*}\right)$. Thus $\hat{\kappa}\left(\mathrm{Ch}\left(C^{*}\right)(w) / S(N)(w)\right)$ describes the image $\hat{\imath}(\hat{v})(\mathfrak{f}(\hat{v})) \cong \mathfrak{f}(\hat{v}) / S\left(N_{W}\right)(\hat{v})$ of $\mathfrak{f}(\hat{v})$ by the projection $\hat{\imath}(\hat{v}): S^{2}\left(V(\hat{v})^{*}\right) \rightarrow S^{2}\left(E(\hat{v})^{*}\right)$.

On the other hand, we have the following commutative diagram:

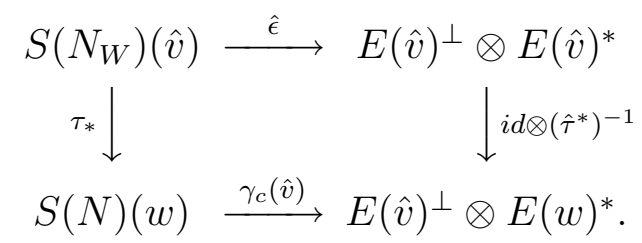

where $\hat{\epsilon}: S\left(N_{W}\right)(\hat{v}) \rightarrow E(\hat{v})^{\perp} \otimes E(\hat{v})^{*}$ is given by $\hat{\epsilon}(f)(v)=[f, v] \in E(\hat{v})^{\perp} \subset V(\hat{v})^{*}=\mathfrak{s}_{-2}(\hat{v})$ in $\mathfrak{s}(\hat{v})$ for $f \in S\left(N_{W}\right)(\hat{v})=\mathfrak{f}(\hat{v}) \cap\left(E(\hat{v})^{\perp} \otimes_{S} V(\hat{v})^{*}\right) \subset S^{2}\left(V(\hat{v})^{*}\right)$ and $v \in E(\hat{v}) \subset \mathfrak{s}_{-1}(\hat{v})$ (see the proof of Lemma $3.1(4))$. Here we note that $\operatorname{Ker} \hat{\epsilon}=S^{2}\left(E(\hat{v})^{\perp}\right)=\operatorname{Ker} \tau_{*}$. Thus $\gamma_{c}(\hat{v})(S(N)(w))$ describes the structure of $S\left(N_{W}\right)(\hat{v})=\mathfrak{f}(\hat{v}) \cap \operatorname{Ker} \hat{\imath}(\hat{v})$.

\section{Second Reduction Theorem}

5.1. Equivalence of $\left(R ; D^{1}, D^{2}\right)$ and $\left(W ; C^{*} . N\right)$. As in $\S 3.1$, let $\left(R ; D^{1}, D^{2}\right)$ be a $P D$ manifold of second order, which is regular of type $\mathfrak{s}$, where $\mathfrak{s}=\mathfrak{s}_{-3} \oplus \mathfrak{s}_{-2} \oplus \mathfrak{s}_{-1}$ is a subalgebra of $\mathfrak{c}^{2}(n)$, which is defined by

$$
\mathfrak{s}_{-3}=\mathbb{R}, \quad \mathfrak{s}_{-2}=V^{*}, \quad \mathfrak{s}_{-1}=V \oplus \mathfrak{f}, \quad \mathfrak{f} \subset S^{2}\left(V^{*}\right) .
$$

We assume that $R$ is regular with respect to $\mathrm{Ch}\left(D^{1}\right)$ and let $p: R \rightarrow J=R / \mathrm{Ch}\left(D^{1}\right)$ be the projection. 
Now we assume that there exists a $G_{0}(\mathfrak{s})$-invariant subspace $E$ of $V$ of dimension $r$. Then, as in $\S 3.1$, we have the first order covariant system $\tilde{N}(E)$ and covariant systems $\tilde{N}^{\perp}(E)$ and $\tilde{N}^{*}(E)$. Moreover, as in $\S 3.2$, let $\left(W ; C^{*}, N\right)$ be the IG manifold of corank $r$ associated with $\left(R ; D^{1}, D^{2}, \tilde{N}(E)\right)$. Namely we consider the map $\eta: R \rightarrow I^{r}(J)$ defined by

$$
\eta(v)=p_{*}(\tilde{N}(v)) \in I^{r}(J) \quad \text { for } \quad v \in R,
$$

where $I^{r}(J)$ is the Involutive Grassmann bundle of $(J, C)$ of codimension $r$. We assume that $\operatorname{Ker} \eta_{*}=\mathrm{Ch}\left(D^{1}\right) \cap \mathrm{Ch}(\tilde{N})$ is a subbundle such that $W=\operatorname{Im}(\eta)$ is a submanifold of $I^{r}(J)$. Let $R(W)$ be the Lagrange Grassmann bundle over $\left(W ; C^{*}, N\right)$. Then we have the map $\kappa_{1}: R \rightarrow$ $R(W)$ defined by

$$
\kappa_{1}(v)=\eta_{*}\left(D^{2}(v)\right) \in R_{w}, \quad w=\eta(v) .
$$

In fact $\hat{v}=\eta_{*}\left(D^{2}(v)\right)$ is a subspace of $N(w) \subset T_{w}(W)$ of dimension $n+t$ such that $\left.\gamma\right|_{\hat{v}}=0$, which follows from $D^{2}(v) \subset \tilde{N}(v), \mathrm{Ch}\left(D^{1}\right)$ is a subbundle of $D^{2}$ of codimension $n$ and $\partial D^{2} \subset D^{1}$. Moreover, by Realization Lemma for $\left(R, D^{2}, \eta, W\right)$, we have $\operatorname{Ker}\left(\kappa_{1}\right)_{*}=\operatorname{Ch}\left(D^{2}\right) \cap \operatorname{Ker} \eta_{*} \subset$ $\mathrm{Ch}\left(D^{1}\right) \cap \operatorname{Ch}\left(D^{2}\right)=\{0\}$, which implies $\operatorname{Ker}\left(\kappa_{1}\right)_{*}$ is trivial, i.e., $\kappa_{1}$ is an immersion such that $\eta=\tau \cdot \kappa_{1}$, where $\tau: R(W) \rightarrow W$ is the projection. Thus, by the definitions of $D_{W}^{1}$ and $D_{W}^{2}$ on $R(W)$, we obtain

Proposition 5.1. $\kappa_{1}:\left(R ; D^{1}, D^{2}\right) \rightarrow\left(R(W) ; D_{W}^{1}, D_{W}^{2}\right)$ is a local isomorphism if and only if

$$
\operatorname{rank} C h\left(D^{1}\right) \cap C h(\tilde{N})=\frac{1}{2}(n-r)(n-r+1) .
$$

When $\kappa_{1}:\left(R ; D^{1}, D^{2}\right) \rightarrow\left(R(W) ; D_{W}^{1}, D_{W}^{2}\right)$ is a local isomorphism, by the construction of $\left(R(W) ; D_{W}^{1}, D_{W}^{2}\right)$ from $\left(W ; C^{*}, N\right)$, we see that the local equivalence of $\left(R ; D^{1}, D^{2}\right)$ is reducible to that of $\left(W ; C^{*}, N\right)$ as in the following: Let $\left(R ; D^{1}, D^{2}\right)$ and $\left(\hat{R} ; \hat{D}^{1}, \hat{D}^{2}\right)$ be $P D$ manifolds of second order, which are regular of type $\mathfrak{s}$, and let $\left(W ; C^{*}, N\right)$ and $\left(\hat{W} ; \hat{C}^{*}, \hat{N}\right)$ be the associated IG manifolds of $\left(R ; D^{1}, D^{2}, \tilde{N}(E)\right)$ and $\left(\hat{R} ; \hat{D}^{1}, \hat{D}^{2}, \hat{N}(E)\right)$ respectively. Moreover let $\left(R(W) ; D_{W}^{1}, D_{W}^{2}\right)$ and $\left(R(\hat{W}) ; D_{\hat{W}}^{1}, D_{\hat{W}}^{2}\right)$ be the Lagrange Grassmann bundle over $\left(W ; C^{*}, N\right)$ and $\left(\hat{W} ; \hat{C}^{*}, \hat{N}\right)$ respectively. For points $v_{o} \in R$ and $\hat{v}_{o} \in \hat{R}$, put $w_{o}=\eta\left(v_{o}\right)$ and $\hat{w}_{o}=\hat{\eta}\left(\hat{v}_{o}\right)$. We assume that $\kappa_{1}:\left(R ; D^{1}, D^{2}\right) \rightarrow\left(R(W) ; D_{W}^{1}, D_{W}^{2}\right)$ and $\hat{\kappa}_{1} ;\left(\hat{R} ; \hat{D}^{1}, \hat{D}^{2}\right) \rightarrow\left(R(\hat{W}) ; D_{\hat{W}}^{1}, D_{\hat{W}}^{2}\right)$ are local isomorphisms around $v_{o}$ and $\hat{v}_{o}$ respectively. Then a local isomorphism $\psi:\left(R ; D^{1}, D^{2}\right) \rightarrow$ $\left(\hat{R}, \hat{D}^{1}, \hat{D}^{2}\right)$ such that $\psi\left(v_{o}\right)=\hat{v}_{o}$ induces a local isomorphism $\varphi:\left(W ; C^{*}, N\right) \rightarrow\left(\hat{W} ; \hat{C}^{*}, \hat{N}\right)$ such that $\varphi\left(w_{o}\right)=\hat{w}_{o}$ and $\varphi_{*}\left(\kappa_{1}\left(v_{o}\right)\right)=\hat{\kappa}_{1}\left(\hat{v}_{o}\right)$, and vice versa.

Moreover, when $\kappa_{1}:\left(R ; D^{1}, D^{2}\right) \rightarrow\left(R(W) ; D_{W}^{1}, D_{W}^{2}\right)$ is a local isomorphism, we observe here the correspondence of local integral manifolds of $\left(R ; D^{1}, D^{2}\right)$ and $\left(W ; C^{*}, N\right)$ as in the following: First we observe, by the uniqueness of the map in the Realization Lemma for $\left(R, D^{2}, p, J\right)$, that the map $\tilde{\kappa}=\zeta \cdot \kappa_{1}: R \rightarrow L(J)$ coincides with the canonical immersion $\iota: R \rightarrow L(J)$, from $\pi \cdot \tilde{\kappa}=q \cdot \tau \cdot \kappa_{1}=q \cdot \eta=p$ and $\tilde{\kappa}_{*}^{-1}(E)=\left(\kappa_{1}\right)_{*}^{-1}\left(D_{W}^{2}\right)=D^{2}$, where $\pi: L(J) \rightarrow J$ is the projection.

Let $\Lambda$ be an integral manifold of $\left(R ; D^{1}, D^{2}\right)$ passing through $v_{o}$, i.e., $\Lambda$ is an $n$-dimensional integral manifold of $\left(R, D^{2}\right)$ such that $T_{v}(\Lambda) \cap \operatorname{Ch}\left(D^{1}\right)(v)=\{0\}$ for $v \in \Lambda$. Then we have $T_{v}(\Lambda) \cap \operatorname{Ker} \eta_{*}(v)=\{o\}$. Hence, from $\tilde{N} \supset D^{2}, \Lambda^{\prime}=\eta(\Lambda)$ is, at least locally, an $n$ dimensional integral manifold of $\left(W ; C^{*}, N\right)$ passing through $w_{o}=\eta\left(v_{o}\right)$ such that $\kappa_{1}(v)=$ $T_{w}\left(\Lambda^{\prime}\right) \oplus \mathrm{Ch}\left(C^{*}\right)(w)$ for $v \in \Lambda$ and $w=\eta(v)$. Conversely let $\Lambda^{\prime}$ be an $n$-dimensional integral manifold of $\left(W ; C^{*}, N\right)$ passing through $w_{o}=\eta\left(v_{o}\right)$ such that $T_{w_{o}}\left(\Lambda^{\prime}\right) \oplus \operatorname{Ch}\left(C^{*}\right)\left(w_{o}\right)=\kappa_{1}\left(v_{o}\right)$, i.e., $\Lambda^{\prime}$ is an $n$-dimensional integral manifold of $(W, N)$ such that $T_{w}\left(\Lambda^{\prime}\right) \cap \mathrm{Ch}\left(C^{*}\right)(w)=\{0\}$ for $w \in \Lambda^{\prime}$ and $T_{w_{o}}\left(\Lambda^{\prime}\right) \oplus \mathrm{Ch}\left(C^{*}\right)\left(w_{o}\right)=\kappa_{1}\left(v_{o}\right)$. Then, from $N \subset C^{*}, \Lambda^{o}=q\left(\Lambda^{\prime}\right)$ is, at least locally, a legendrian submanifold of $(J, C)$. Hence we have the lift $\sigma\left(\Lambda^{o}\right) \subset L(J)$ of $\Lambda^{o}$ 
by $\sigma(u)=T_{u}\left(\Lambda^{o}\right) \in L(J)$ for $u \in \Lambda^{o}$. Moreover we have a map $\lambda: \Lambda^{\prime} \rightarrow R(W)$ defined by $\lambda(w)=T_{w}\left(\Lambda^{\prime}\right) \oplus \operatorname{Ch}\left(C^{*}\right)(w) \in R(W)$. Then, from $\tau \cdot \lambda=i d_{\Lambda^{\prime}}$ and by the definition of $\zeta: R(W) \rightarrow L(J)$, we see that $\lambda$ is an immersion such that $\lambda\left(w_{o}\right)=\kappa_{1}\left(v_{o}\right)$ and $\zeta \cdot \lambda(w)=\sigma \cdot q(w)$ for $w \in \Lambda^{\prime}$. Hence $\Lambda=\left(\kappa_{1}\right)^{-1}\left(\lambda\left(\Lambda^{\prime}\right)\right)$ is an integral manifold of $\left(R ; D^{1}, D^{2}\right)$ passing through $v_{o} \in R$ such that $\eta(\Lambda)=\tau \cdot \kappa_{1}(\Lambda)=\Lambda^{\prime}$ and $\iota(\Lambda)=\zeta \cdot \kappa_{1}(\Lambda)=\zeta \cdot \lambda\left(\Lambda^{\prime}\right)=\sigma \cdot q\left(\Lambda^{\prime}\right)=\sigma\left(\Lambda^{o}\right)$ around $v_{o} \in R$.

5.2. Covariant systems $\mathfrak{f}(E)$ and $C(E)$. We will first consider the condition in Proposition 5.1 in terms of the symbol algebra $\mathfrak{s}$ of $\left(R ; D^{1}, D^{2}\right)$. Here $\left(R ; D^{1}, D^{2}\right)$ is regular of type $\mathfrak{s}$. Namely the symbol algebra $\mathfrak{s}(v)=\mathfrak{s}_{-3}(v) \oplus \mathfrak{s}_{-2}(v) \oplus \mathfrak{s}_{-1}(v)$ at each $v \in R$ is isomorphic to $\mathfrak{s}=\mathfrak{s}_{-3} \oplus \mathfrak{s}_{-2} \oplus \mathfrak{s}_{-1}$. We define subspaces $\mathfrak{f}_{E}$ and $\mathfrak{c}_{E}$ of $\mathfrak{f}$ and $\mathfrak{s}_{-1}$ by

$$
\mathfrak{f}_{E}=\mathfrak{f} \cap S^{2}\left(E^{\perp}\right) \subset \mathfrak{f}, \quad \mathfrak{c}_{E}=\hat{E} \oplus \mathfrak{f}_{E} \subset \mathfrak{s}_{-1}=V \oplus \mathfrak{f},
$$

where $\hat{E}=\left\{v \in E \mid v \odot E \subset \mathfrak{f}^{\perp}\right\}$. Then we have

Lemma 5.1. $\mathfrak{f}_{E}$ and $\mathfrak{c}_{E}$ are $G(\mathfrak{s})$-invariant.

Proof. Since $E$ is $G_{o}(\mathfrak{s})$-invariant, $\hat{E}$ and $E^{\perp}$ are $G_{0}(\mathfrak{s})$-invariant subspaces of $V$ and $V^{*}$ respectively, which also implies $S^{2}\left(E^{\perp}\right)$ is a $G_{0}(\mathfrak{s})$-invariant subspace of $S^{2}\left(V^{*}\right)$. Hence $\mathfrak{f}_{E}$ is a $G(\mathfrak{s})$-invariant subspace of $\mathfrak{f}$. Since $\hat{E}$ is $G_{o}(\mathfrak{s})$-invariant, to show that $\mathfrak{c}_{E}$ is $G(\mathfrak{s})$-invariant, it suffices to check that $\rho(\hat{E}) \subset \mathfrak{f}_{E}$ for each $\rho \in \mathfrak{f}^{(1)}$, where $\mathfrak{f}^{(1)}=\mathfrak{f} \otimes V^{*} \cap S^{3}\left(V^{*}\right)$ is the first prolongation of $\mathfrak{f}$ and $\rho: V \rightarrow \mathfrak{f} \subset S^{2}\left(V^{*}\right)$ satisfies $\left.\left.v_{1}\right\rfloor \rho\left(v_{2}\right)=v_{2}\right\rfloor \rho\left(v_{1}\right)$ (see $\S 5.2$ [20] for the detail). From $\left(S^{2}\left(E^{\perp}\right)\right)^{\perp}=E \otimes_{S} V \subset S^{2}(V)$, we have $\left(\mathfrak{f}_{E}\right)^{\perp}=\mathfrak{f}^{\perp}+E \otimes_{S} V$. Hence, from $\hat{E} \otimes_{S} E \subset \mathfrak{f}^{\perp}, \rho(\hat{E}) \subset \mathfrak{f}_{E}$ follows from

$$
\left.\mathfrak{f}^{\perp}+E \otimes_{S} V \subset(\hat{E}\rfloor \mathfrak{f}^{(1)}\right)^{\perp}=\left\{a \in S^{2}(V) \mid \hat{E} \odot a \subset\left(\mathfrak{f}^{(1)}\right)^{\perp}\right\} .
$$

Hence we can define the covariant systems $\mathfrak{f}(E)$ and $C(E)$ of $\left(R ; D^{1}, D^{2}\right)$ as follows; Take a graded Lie algebra isomorphism $\phi$ of $\mathfrak{s}(v)$ onto $\mathfrak{s}$. We put

$$
\mathfrak{f}(E)(v)=\phi^{-1}\left(\mathfrak{f}_{E}\right) \subset C(E)(v)=\phi^{-1}\left(\mathfrak{c}_{E}\right) \subset D^{2}(v)=\mathfrak{s}_{-1}(v) .
$$

Then, by the above lemma, it follows that $\mathfrak{f}(E)(v)$ and $C(E)(v)$ are well-defined and we obtain subbundles $\mathfrak{f}(E)$ and $C(E)$ of $D^{2}$.

Moreover, starting from the $G(\mathfrak{s})$-invariant subspace $\hat{E}$, we put

$$
\mathfrak{f}_{\hat{E}}=\mathfrak{f} \cap S^{2}\left((\hat{E})^{\perp}\right) \subset \mathfrak{f}, \quad \mathfrak{c}_{\hat{E}}=\hat{E} \oplus \mathfrak{f}_{\hat{E}} \subset \mathfrak{s}_{-1}=V \oplus \mathfrak{f} .
$$

$\mathfrak{f}_{\hat{E}}$ and $\mathfrak{c}_{\hat{E}}$ are also $G(\mathfrak{s})$-invariant and we can define the covariant systems $\mathfrak{f}(\hat{E})$ and $C(\hat{E})$ of $\left(R ; D^{1} \cdot D^{2}\right)$ as follows;

$$
\mathfrak{f}(\hat{E})(v)=\phi^{-1}\left(\mathfrak{f}_{\hat{E}}\right) \subset C(\hat{E})(v)=\phi^{-1}\left(\mathfrak{c}_{\hat{E}}\right) \subset D^{2}(v)=\mathfrak{s}_{-1}(v),
$$

for a graded Lie algebra isomorphism $\phi$ of $\mathfrak{s}(v)$ onto $\mathfrak{s}$. Here we note $\hat{(} \hat{E})=\hat{E}$.

For these systems, we have

Lemma 5.2. (1) $\mathfrak{f}(E)=C h\left(D^{1}\right) \cap C h(\tilde{N})$, if $C(E)=C h(\tilde{N})$.

(2) $\quad C h\left(D^{1}\right)(v) \cap C h(\tilde{N})(v) \subset \mathfrak{f}(E)(v)$ and $C h(\tilde{N})(v) \subset C(E)(v)$ at each $v \in R$.

(3) $C h\left(D^{1}\right)(v) \cap C h\left(\tilde{N}^{*}\right)(v) \subset \mathfrak{f}(\hat{E})(v)$ and $C h\left(\tilde{N}^{*}\right)(v) \subset C(\hat{E})(v)$ at each $v \in R$.

(4) $\operatorname{rank} C h\left(D^{1}\right) \cap C h(\tilde{N})=\frac{1}{2}(n-r)(n-r+1)$ iff $\mathfrak{f}^{\perp} \subset E \otimes_{S} V$ and $C h\left(D^{1}\right) \cap C h(\tilde{N})=\mathfrak{f}(E)$.

(5) If $\mathfrak{f}^{\perp} \subset E \otimes_{S} V$, then $C h\left(D^{2}\right)(v) \subset C(E)(v)$ at each $v \in R$. 
Proof. (1) By definition, $\mathfrak{f}(E)=C(E) \cap \mathrm{Ch}\left(D^{1}\right)$.

(2) By Lemma 3.1, Ch $(\tilde{N})(v) \subset D^{2}(v)=\mathfrak{s}_{-1}(v)$. Let $\phi$ be a graded Lie algebra isomorphism of $\mathfrak{s}(v)$ onto $\mathfrak{s}$. For a vector $X \in \operatorname{Ch}(\tilde{N})(v)$, we have $[X, \Gamma(\tilde{N})] \subset \Gamma(\tilde{N})$. Moreover $\tilde{N}(v)$ corresponds to the subspace $E^{\perp} \oplus \mathfrak{s}_{-1}$ of $\mathfrak{s}$ under $\phi$ (see $\S 3.1$ ). Thus $\phi(X) \in \mathfrak{s}_{-1}$ satisfies $\left[\phi(X), \mathfrak{s}_{-1}\right] \subset E^{\perp}$ and $\left[\phi(X), E^{\perp}\right]=0$. Then, for $\phi(X)=v+a, v \in V, a \in \mathfrak{f}$, we have $\left.v\rfloor \mathfrak{f} \subset E^{\perp}, V\right\rfloor a \subset E^{\perp}$ and $\left\langle v, E^{\perp}\right\rangle=0$. Hence we have $v \in E, v \odot E \subset \mathfrak{f}^{\perp}$ and $\left.X\right\rfloor a=0$ for $\forall X \in E$, which implies $v \in \hat{E}$ and $a \in \mathfrak{f}_{E}$.

(3) can be shown similarly as in (2) by taking $\hat{E}$ in place of $E$.

(4) By $\mathfrak{f}_{E}=\mathfrak{f} \cap S^{2}\left(E^{\perp}\right), \operatorname{dim} \mathfrak{f}_{E} \leqq \frac{1}{2}(n-r)(n-r+1)$ and the equality holds iff $\mathfrak{f}_{E}=S^{2}\left(E^{\perp}\right)$, i.e., iff $\mathfrak{f} \supset S^{2}\left(E^{\perp}\right)$. Thus (4) follows immediately from (2).

(5) For a vector $X \in \mathrm{Ch}\left(D^{2}\right)(v)$, we have $\left[X, \Gamma\left(D^{2}\right)\right] \subset \Gamma\left(D^{2}\right)$. Hence we have $\left[\phi(X), \mathfrak{s}_{-1}\right]=0$ for an isomorphism $\phi: \mathfrak{s}(v) \rightarrow \mathfrak{s}$. Thus, for $\phi(X)=v+a, v \in V, a \in \mathfrak{f}$, we have $v\rfloor \mathfrak{f}=0$ and $V\rfloor a=0$. Hence, from $\mathfrak{f} \supset S^{2}\left(E^{\perp}\right)$, we have $v \in E, v \odot E \subset \mathfrak{f}^{\perp}$ and $a=0$, which implies $\phi(X) \in \hat{E}$. This completes the proof of $(5)$.

Now we assume that there exists $G_{0}(\mathfrak{s})$-invariant subspace $E$ of $V$ of dimension $r$ such that $\mathfrak{f}^{\perp} \subset E \otimes_{S} V$. Let $s$ be the dimension of $\hat{E}=\left\{v \in E \mid v \odot E \subset \mathfrak{f}^{\perp}\right\}$. First we describe the structure equation of the graded Lie algebra $\mathfrak{s}=\mathfrak{s}_{-3} \oplus \mathfrak{s}_{-2} \oplus \mathfrak{s}_{-1}$, where

$$
\mathfrak{s}_{-3}=\mathbb{R}, \quad \mathfrak{s}_{-2}=V^{*}, \quad \mathfrak{s}_{-1}=V \oplus \mathfrak{f}, \quad \mathfrak{f} \subset S^{2}\left(V^{*}\right) .
$$

We have $\hat{E} \otimes_{S} E \subset \mathfrak{f}^{\perp} \subset E \otimes_{S} V$. Thus we have a basis $\left\{e_{1}, \ldots, e_{n}\right\}$ of $V$ such that

$$
\begin{gathered}
\hat{E}=\left\langle\left\{e_{1}, \ldots, e_{s}\right\}\right\rangle \subset E=\left\langle\left\{e_{1}, \ldots, e_{r}\right\}\right\rangle \\
\left\langle\left\{e_{a} \odot e_{k}(1 \leqq a \leqq s, 1 \leqq k \leqq r)\right\}\right\rangle \subset \mathfrak{f}^{\perp} \subset\left\langle\left\{e_{i} \odot e_{\ell}(1 \leqq i \leqq r, 1 \leqq \ell \leqq n)\right\}\right\rangle .
\end{gathered}
$$

Namely we have

$$
\begin{aligned}
S^{2}\left(E^{\perp}\right)= & \left\langle\left\{e_{\alpha}^{*} \odot e_{\beta}^{*}(r+1 \leqq \alpha \leqq \beta \leqq n)\right\}\right\rangle \subset \mathfrak{f} \subset \\
& S^{2}\left(E^{\perp}\right) \cup\left\langle\left\{e_{k}^{*} \odot e_{\alpha}^{*}(1 \leqq k \leqq r, r+1 \leqq \alpha \leqq n)\right\}\right\rangle \cup\left\langle\left\{e_{i}^{*} \odot e_{j}^{*}(s+1 \leqq i \leqq j \leqq r)\right\}\right\rangle,
\end{aligned}
$$

where $\left\{e_{1}^{*}, \ldots, e_{n}^{*}\right\}$ are the dual basis of $\left\{e_{1}, \ldots, e_{n}\right\}$ in $V^{*}$. Let us take the complimentary subspace $H$ of $V$ such that $V=E \oplus H$, where $H=\left\langle\left\{e_{r+1}, \ldots, e_{n}\right\}\right\rangle$, and take a complimentary subspace $T$ of $E \otimes_{S} V$ such that $E \otimes_{S} V=\mathfrak{f}^{\perp} \oplus T$. Then, from $S^{2}(V)=E \otimes_{S} V \oplus S^{2}(H)$, $\operatorname{dim} T=\operatorname{dim} \mathfrak{f}-\operatorname{dim} S^{2}\left(E^{\perp}\right)(=t)$. Let $\left\{\tilde{\pi}_{\lambda}(1 \leqq \lambda \leqq t)\right\}$ be a basis of $T$ and put $\tilde{\pi}_{k l}=e_{k} \odot e_{l}$ $(1 \leqq k \leqq r, 1 \leqq l \leqq n)$ and $\tilde{\varpi}_{\alpha \beta}=e_{\alpha} \odot e_{\beta}(1 \leqq \alpha, \beta \leqq n)$. Under the identification: $S^{2}(V) \cong\left(S^{2}\left(V^{*}\right)\right)^{*}$, we restrict these covectors to the subspace $\mathfrak{f} \subset S^{2}\left(V^{*}\right)$ and put

$$
\hat{\pi}_{\lambda}=\left.\tilde{\pi}_{\lambda}\right|_{\mathfrak{f}}, \quad \hat{\pi}_{k l}=\left.\tilde{\pi}_{k l}\right|_{\mathfrak{f}} \quad \text { and } \quad \hat{\varpi}_{\alpha \beta}=\left.\tilde{\varpi}_{\alpha \beta}\right|_{\mathfrak{f}} .
$$

Then we have $\hat{\pi}_{a k}=0(1 \leqq a \leqq s, 1 \leqq k \leqq r)$ and $\left\{\hat{\pi}_{\lambda}(1 \leqq \lambda \leqq t)\right\}$ forms a basis of $\left\langle\left\{\hat{\pi}_{k, \alpha}(1 \leqq\right.\right.$ $\left.\left.k \leqq r, r+1 \leqq \alpha \leqq n), \hat{\pi}_{i j}(s+1 \leqq i, j \leqq r)\right\}\right\rangle$. Moreover $\left\{\hat{\pi}_{\lambda}(1 \leqq \lambda \leqq t), \hat{\varpi}_{\alpha \beta}(1 \leqq \alpha \leqq \beta \leqq n)\right\}$ forms a basis of $\mathfrak{f}^{*}$. Then, firstly fixing a basis of $\mathfrak{s}_{-3}$, we have a basis of $\mathfrak{s}=\mathfrak{s}_{-3} \oplus \mathfrak{s}_{-2} \oplus \mathfrak{s}_{-1}$, $\mathfrak{s}_{-3} \cong \mathbb{R}, \mathfrak{s}_{-2} \cong V^{*}$ and $\mathfrak{s}_{-1}=V \oplus \mathfrak{f}$, by fixing the basis $\left\{e_{1}, \ldots, e_{n}\right\}$ of $V$ as above. Thus we have covectors

$$
\begin{aligned}
\left\{\hat{\varpi}, \hat{\varpi}_{1}, \ldots, \hat{\varpi}_{n}, \hat{\omega}^{1}, \ldots, \hat{\omega}^{n}, \hat{\varpi}_{\alpha \beta}(r+1 \leqq \alpha, \beta \leqq n),\right. \\
\left.\hat{\pi}_{k, \alpha}(1 \leqq k \leqq r, r+1 \leqq \alpha \leqq n), \hat{\pi}_{i j}(s+1 \leqq i, j \leqq r)\right\}
\end{aligned}
$$


in $\mathfrak{s}^{*}$ such that $\hat{\varpi}_{\alpha \beta}=\hat{\varpi}_{\beta \alpha}, \hat{\pi}_{i j}=\hat{\pi}_{j i}$, and that

$$
\left\{\begin{array}{l}
d \hat{\varpi}=\hat{\omega}^{1} \wedge \hat{\varpi}_{1}+\cdots+\hat{\omega}^{n} \wedge \hat{\varpi}_{n}, \\
d \hat{\varpi}_{a}=\quad \sum_{\alpha=r+1}^{n} \hat{\omega}^{\alpha} \wedge \hat{\pi}_{a \alpha} \quad(1 \leqq a \leqq s), \\
d \hat{\varpi}_{i}=\quad \sum_{j=s+1}^{r} \hat{\omega}^{j} \wedge \hat{\pi}_{i j}+\sum_{\alpha=r+1}^{n} \hat{\omega}^{\alpha} \wedge \hat{\pi}_{i \alpha} \quad(s+1 \leqq i \leqq r), \\
d \hat{\varpi}_{\alpha}=\sum_{a=1}^{s} \hat{\omega}^{a} \wedge \hat{\pi}_{a \alpha}+\sum_{i=s+1}^{r} \hat{\omega}^{i} \wedge \hat{\pi}_{i \alpha}+\sum_{\beta=r+1}^{n} \hat{\omega}^{\beta} \wedge \hat{\varpi}_{\alpha \beta} \quad(r+1 \leqq \alpha \leqq n),
\end{array}\right.
$$

where $\left\{\hat{\varpi}, \hat{\varpi}_{1}, \ldots, \hat{\varpi}_{n}, \hat{\omega}^{1}, \ldots, \hat{\omega}^{n}, \hat{\varpi}_{\alpha \beta}(r+1 \leqq \alpha \leqq \beta \leqq n), \hat{\pi}_{\lambda}(1 \leqq \lambda \leqq t)\right\}$ forms a basis of $\mathfrak{s}^{*}$ such that $\left\{\hat{\pi}_{\lambda}(1 \leqq \lambda \leqq t)\right\}$ is a basis of $\left\langle\left\{\hat{\pi}_{k, \alpha}(1 \leqq k \leqq r, r+1 \leqq \alpha \leqq n), \hat{\pi}_{i j}(s+1 \leqq\right.\right.$ $i, j \leqq r)\}\rangle$. Moreover, putting $\hat{\Omega}_{i}=\sum_{j=s+1}^{r} \hat{\omega}^{j} \wedge \hat{\pi}_{i j}$, we see that $\left\{\hat{\Omega}_{s+1}, \ldots, \hat{\Omega}_{r}\right\}$ are linearly independent as follows; If $\sum_{i=s+1}^{r} a_{i} \hat{\Omega}_{i}=0$, we have $\sum_{i=s+1}^{r} a_{i} \hat{\pi}_{i j}=0$ for $s+1 \leqq j \leqq r$. From $\hat{\pi}_{i a}=0(s+1 \leqq i \leqq r, 1 \leqq a \leqq s)$, we have $\sum_{i=s+1}^{r} a_{i} \hat{\pi}_{i k}=0$ for $1 \leqq k \leqq r$, which implies $\left(\sum_{i=s+1}^{r} a_{i} e_{i}\right) \odot e_{k} \in \mathfrak{f}^{\perp}$ for $1 \leqq k \leqq r$. Then by the definition of $\hat{E}$, we get $\sum_{i=s+1}^{r} a_{i} e_{i} \in \hat{E}$, which shows $a_{s+1}=\cdots=a_{r}=0$, by the choice of our basis.

Now let $\left(R ; D^{1}, D^{2}\right)$ be a $P D$ manifold of second order, which is regular of type $\mathfrak{s}$. Let us fix a point $v \in R$. Then, as in $\S 1$ of [23], there exists a coframe $\left\{\varpi, \varpi_{1}, \ldots, \varpi_{n}, \omega^{1}, \ldots, \omega^{n}, \varpi_{\alpha \beta}(r+\right.$ $\left.1 \leqq \alpha \leqq \beta \leqq n), \pi_{\lambda}(1 \leqq \lambda \leqq t)\right\}\left(t=\operatorname{dim} \mathfrak{f}-\operatorname{dim} S^{2}\left(E^{\perp}\right)\right)$ and 1 -forms $\left\{\pi_{k, \alpha}(1 \leqq k \leqq r, r+1 \leqq\right.$ $\left.\alpha \leqq n), \pi_{i j}(s+1 \leqq i, j \leqq r)\right\}$ defined around $v \in R$ such that

$D^{1}=\{\varpi=0\}, \quad D^{2}=\left\{\varpi=\varpi_{1}=\cdots=\varpi_{n}=0\right\}, \quad \tilde{N}=\tilde{N}(E)=\left\{\varpi=\varpi_{1}=\cdots=\varpi_{r}=0\right\}$, $\tilde{N}^{*}=\tilde{N}(\hat{E})=\left\{\varpi=\varpi_{1}=\cdots=\varpi_{s}=0\right\}, \quad \tilde{N}^{\perp}=\left\{\varpi=\varpi_{1}=\cdots=\varpi_{n}=\omega^{r+1}=\cdots=\omega^{n}=0\right\}$, and the following structure equations hold;

$$
\begin{gathered}
d \varpi \equiv \omega^{1} \wedge \varpi_{1}+\cdots+\omega^{n} \wedge \varpi_{n} \quad(\bmod \varpi), \\
d \varpi_{a} \equiv \quad \sum_{\alpha=r+1}^{n} \omega^{\alpha} \wedge \pi_{a \alpha}(1 \leqq a \leqq s), \\
d \varpi_{i} \equiv \quad \sum_{j=s+1}^{r} \omega^{j} \wedge \pi_{i j}+\sum_{\alpha=r+1}^{n} \omega^{\alpha} \wedge \pi_{i \alpha}(s+1 \leqq i \leqq r), \\
d \varpi_{\alpha} \equiv \sum_{a=1}^{s} \omega^{a} \wedge \pi_{a \alpha}+\sum_{i=s+1}^{r} \omega^{i} \wedge \pi_{i \alpha}+\sum_{\beta=r+1}^{n} \omega^{\beta} \wedge \varpi_{\alpha \beta}(r+1 \leqq \alpha \leqq n),
\end{gathered}
$$

$\left(\bmod \varpi, \varpi_{1}, \ldots, \varpi_{n}\right)$, where $\varpi_{\alpha \beta}=\varpi_{\beta \alpha}, \pi_{i j}=\pi_{j i}$, and $\left\{\pi_{\lambda}(1 \leqq \lambda \leqq t)\right\}$ is a basis of $\left\langle\left\{\pi_{k, \alpha}(1 \leqq k \leqq r, r+1 \leqq \alpha \leqq n), \pi_{i j}(s+1 \leqq i, j \leqq r)\right\}\right\rangle\left(\bmod \varpi, \varpi_{1}, \ldots, \varpi_{n}\right)$. Moreover $\left\{\Omega_{s+1}, \ldots, \Omega_{r}\right\}$ are linearly independent $\left(\bmod \varpi, \varpi_{1} \ldots, \varpi_{n}\right)$, where $\Omega_{i}=\sum_{j=s+1}^{r} \omega^{j} \wedge \pi_{i j}$. Furthermore, we have

$$
C(E)=\left\{\varpi=\varpi_{1}=\cdots=\varpi_{n}=\omega^{s+1}=\cdots=\omega^{n}=\pi_{1}=\cdots=\pi_{t}=0\right\},
$$

and

$$
\mathfrak{f}(E)=\left\{\varpi=\varpi_{1}=\cdots=\varpi_{n}=\omega^{1}=\cdots=\omega^{n}=\pi_{1}=\cdots=\pi_{t}=0\right\} .
$$

By utilizing the structure equations (A) and (B.1), we will investigate the properties of $C(E)$ and $\mathfrak{f}(E)$. In the rest of this section, we will adopt the Einstein's convention for indices. The 
index ranges are as follows; $1 \leqq a, b \leqq s, s+1 \leqq i, j, k \leqq r, r+1 \leqq \alpha, \beta \leqq n$ and $1 \leqq \lambda \leqq t$. From (B.1), we have

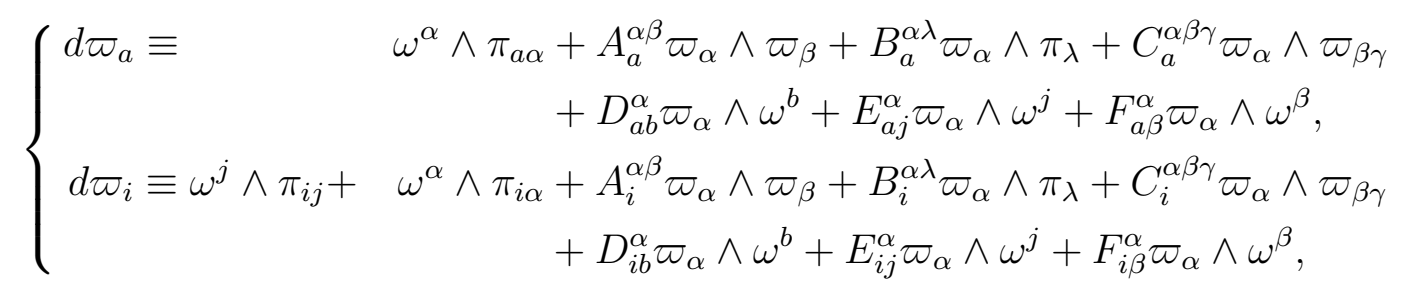

$\left(\bmod \varpi, \varpi_{1}, \ldots, \varpi_{r}\right)$. From $(\mathrm{A})$, we have $d \varpi \equiv 0\left(\bmod \varpi, \varpi_{1}, \ldots, \varpi_{r}, \omega^{r+1}, \ldots, \omega^{n}\right)$. Hence we have

$$
d \omega^{\alpha} \wedge \varpi_{\alpha} \equiv \omega^{a} \wedge d \varpi_{a}+\omega^{i} \wedge d \varpi_{i} \quad\left(\bmod \varpi, \varpi_{1}, \ldots, \varpi_{r}, \omega^{r+1}, \ldots, \omega^{n}\right) .
$$

Then, from (B.2), we get

$$
\begin{aligned}
& d \omega^{\alpha}+\omega^{a} \wedge\left(B_{a}^{\alpha \lambda} \pi_{\lambda}+C_{a}^{\alpha \beta \gamma} \varpi_{\beta \gamma}+D_{a b}^{\alpha} \omega^{b}+E_{a j}^{\alpha} \omega^{j}\right) \\
& \quad+\omega^{i} \wedge\left(B_{i}^{\alpha \lambda} \pi_{\lambda}+C_{i}^{\alpha \beta \gamma} \varpi_{\beta \gamma}+D_{i b}^{\alpha} \omega^{b}+E_{i j}^{\alpha} \omega^{j}\right) \equiv 0\left(\bmod \varpi, \varpi_{1}, \ldots, \varpi_{n}, \omega^{r+1}, \ldots, \omega^{n}\right) .
\end{aligned}
$$

Putting $\mathfrak{M}_{1}=\left\langle\left\{\varpi, \varpi_{1}, \ldots, \varpi_{n}, \pi_{1}, \ldots, \pi_{t}\right\}\right\rangle=\left\langle\left\{\varpi, \varpi_{1}, \ldots, \varpi_{n}, \pi_{k, \alpha}(1 \leqq k \leqq r, r+1 \leqq \alpha \leqq\right.\right.$ $\left.\left.n), \pi_{i j}(s+1 \leqq i, j \leqq r)\right\}\right\rangle$, we have

$$
d \varpi \equiv d \varpi_{a} \equiv d \varpi_{i} \equiv 0, \quad d \varpi_{\alpha} \equiv \omega^{\delta} \wedge \varpi_{\alpha \delta} \quad\left(\bmod \quad \mathfrak{M}_{1}\right) .
$$

Then, taking exterior derivatives of both sides of (B.2) and calculating mod $\mathfrak{M}_{1}$, we get

$$
\omega^{\alpha} \wedge d \pi_{a \alpha} \equiv \omega^{\delta} \wedge \varpi_{\alpha \delta} \wedge\left(C_{a}^{\alpha \beta \gamma} \varpi_{\beta \gamma}+D_{a b}^{\alpha} \omega^{b}+E_{a j}^{\alpha} \omega^{j}+F_{a \beta}^{\alpha} \omega^{\beta}\right) \quad\left(\bmod \quad \mathfrak{M}_{1}\right),
$$

and

$$
\omega^{j} \wedge d \pi_{i j}+\omega^{\alpha} \wedge d \pi_{i \alpha} \equiv \omega^{\delta} \wedge \varpi_{\alpha \delta} \wedge\left(C_{i}^{\alpha \beta \gamma} \varpi_{\beta \gamma}+D_{i b}^{\alpha} \omega^{b}+E_{i j}^{\alpha} \omega^{j}+F_{i \beta}^{\alpha} \omega^{\beta}\right) \quad\left(\bmod \quad \mathfrak{M}_{1}\right) .
$$

Thus we obtain

$$
d \pi_{a \alpha} \equiv C_{a}^{\delta \beta \gamma} \varpi_{\alpha \delta} \wedge \varpi_{\beta \gamma}+D_{a b}^{\delta} \varpi_{\alpha \delta} \wedge \omega^{b}+E_{a j}^{\delta} \varpi_{\alpha \delta} \wedge \omega^{j}
$$

$\left(\bmod \varpi, \varpi_{1}, \ldots, \varpi_{n}, \omega^{r+1}, \ldots, \omega^{n}, \pi_{1}, \ldots, \pi_{t}\right)$, and

$$
\left\{\begin{aligned}
d \pi_{i \alpha} & \equiv C_{i}^{\delta \beta \gamma} \varpi_{\alpha \delta} \wedge \varpi_{\beta \gamma}+D_{i b}^{\delta} \varpi_{\alpha \delta} \wedge \omega^{b}, \\
d \pi_{i j} & \equiv 0 \quad\left(\bmod \varpi, \varpi_{1}, \ldots, \varpi_{n}, \omega^{s+1}, \ldots, \omega^{n}, \pi_{1}, \ldots, \pi_{t}\right) .
\end{aligned}\right.
$$

Moreover, from (B.2), we have, for $s+1 \leqq p, q \leqq n$,

$$
\begin{aligned}
d \varpi_{a} \equiv & \omega^{\alpha} \wedge \pi_{a \alpha}+A_{a}^{p q} \varpi_{p} \wedge \varpi_{q}+B_{a}^{j \lambda} \varpi_{j} \wedge \pi_{\lambda}+B_{a}^{\alpha \lambda} \varpi_{\alpha} \wedge \pi_{\lambda} \\
& +C_{a}^{j \beta \gamma} \varpi_{j} \wedge \varpi_{\beta \gamma}+C_{a}^{\alpha \beta \gamma} \varpi_{\alpha} \wedge \varpi_{\beta \gamma}+D_{a b}^{j} \varpi_{j} \wedge \omega^{b}+D_{a b}^{\alpha} \varpi_{\alpha} \wedge \omega^{b} \\
& +E_{a k}^{j} \varpi_{j} \wedge \omega^{k}+E_{a j}^{\alpha} \varpi_{\alpha} \wedge \omega^{j}+F_{a \beta}^{j} \varpi_{j} \wedge \omega^{\beta}+F_{a \beta}^{\alpha} \varpi_{\alpha} \wedge \omega^{\beta}\left(\bmod \varpi \varpi_{1}, \ldots, \varpi_{s}\right) .
\end{aligned}
$$

From $(\mathrm{A})$, we have $d \varpi \equiv 0\left(\bmod \varpi, \varpi_{1}, \ldots, \varpi_{s}, \omega^{s+1}, \ldots, \omega^{n}\right)$. Hence we have

$$
d \omega^{i} \wedge \varpi_{i}+d \omega^{\alpha} \wedge \varpi_{\alpha} \equiv \omega^{a} \wedge d \varpi_{a} \quad\left(\bmod \varpi, \varpi_{1}, \ldots, \varpi_{s}, \omega^{s+1}, \ldots, \omega^{n}\right) .
$$

Then, from (B.3), we get

$$
d \omega^{i}+\omega^{a} \wedge\left(B_{a}^{i \lambda} \pi_{\lambda}+C_{a}^{i \beta \gamma} \varpi_{\beta \gamma}+D_{a b}^{i} \omega^{b}\right) \equiv 0 \quad\left(\bmod \varpi, \varpi_{1}, \ldots, \varpi_{n}, \omega^{s+1}, \ldots, \omega^{n}\right) .
$$

Here we have

Lemma 5.3. (1) $\mathfrak{f}(E)=C h\left(D^{1}\right) \cap C h(\tilde{N})$ iff $C_{a}^{\alpha \beta \gamma}=C_{i}^{\alpha \beta \gamma}=0$.

(2) $C(E)=C h(\tilde{N})$ iff $C_{a}^{\alpha \beta \gamma}=C_{i}^{\alpha \beta \gamma}=D_{a b}^{\alpha}=D_{i b}^{\alpha}=0$.

(3) $C(E) \subset C h\left(\tilde{N}^{*}\right)$ iff $C_{a}^{\alpha \beta \gamma}=C_{a}^{i \beta \gamma}=D_{a b}^{\alpha}=D_{a b}^{i}=0$. 
Proof. (1) From $C h\left(D^{1}\right) \cap \mathrm{Ch}(\tilde{N}) \subset \mathfrak{f}(E) \subset \mathrm{Ch}\left(D^{1}\right)$, we see that $\mathfrak{f}(E)=\mathrm{Ch}\left(D^{1}\right) \cap \mathrm{Ch}(\tilde{N})$ iff $\mathfrak{f}(E) \subset \mathrm{Ch}(\tilde{N})$. For a vector $X \in \mathfrak{f}(E)(v), v \in R$, by (A) and (B.2), we have

$$
\left\{\begin{array}{rlrl}
X\rfloor d \varpi & \equiv 0 \quad(\bmod \varpi), & \\
X\rfloor d \varpi_{a} & \equiv-C_{a}^{\alpha \beta \gamma} \varpi_{\beta \gamma}(X) \varpi_{\alpha} & & \left(\bmod \varpi, \varpi_{1}, \ldots, \varpi_{r}\right), \\
X\rfloor d \varpi_{i} & \equiv-C_{i}^{\alpha \beta \gamma} \varpi_{\beta \gamma}(X) \varpi_{\alpha} & & \left(\bmod \varpi, \varpi_{1}, \ldots, \varpi_{r}\right) .
\end{array}\right.
$$

Thus $X \in \operatorname{Ch}(\tilde{N})(v)$ iff $C_{a}^{\alpha \beta \gamma} \varpi_{\beta \gamma}(X)=C_{i}^{\alpha \beta \gamma} \varpi_{\beta \gamma}(X)=0$, which completes the proof of (1).

(2) From $\operatorname{Ch}(\tilde{N}) \subset C(E), C(E)=\operatorname{Ch}(\tilde{N})$ iff $C(E) \subset \mathrm{Ch}(\tilde{N})$. For a vector $X \in C(E)(v)$, $v \in R$, by (A) and (B.2), we have

$$
\left\{\begin{aligned}
X\rfloor d \varpi & \equiv \omega^{a}(X) \varpi_{a} \quad(\bmod \varpi) \quad \equiv 0 & & \left(\bmod \varpi, \varpi_{1}, \ldots, \varpi_{r}\right), \\
X\rfloor d \varpi_{a} & \equiv-\left(C_{a}^{\alpha \beta \gamma} \varpi_{\beta \gamma}+D_{a b}^{\alpha} \omega^{b}\right)(X) \varpi_{\alpha} & & \left(\bmod \varpi, \varpi_{1}, \ldots, \varpi_{r}\right), \\
X\rfloor d \varpi_{i} & \equiv-\left(C_{i}^{\alpha \beta \gamma} \varpi_{\beta \gamma}+D_{i b}^{\alpha} \omega^{b}\right)(X) \varpi_{\alpha} & & \left(\bmod \varpi, \varpi_{1}, \ldots, \varpi_{r}\right) .
\end{aligned}\right.
$$

Thus $X \in \operatorname{Ch}(\tilde{N})(v)$ iff $\left(C_{a}^{\alpha \beta \gamma} \varpi_{\beta \gamma}+D_{a b}^{\alpha} \omega^{b}\right)(X)=\left(C_{i}^{\alpha \beta \gamma} \varpi_{\beta \gamma}+D_{i b}^{\alpha} \omega^{b}\right)(X)=0$, which completes the proof of $(2)$.

(3) For a vector $X \in C(E)(v), v \in R$, by (A) and (B.3), we have

$$
\left\{\begin{array}{l}
X\rfloor d \varpi \equiv \omega^{a}(X) \varpi_{a} \quad(\bmod \varpi) \quad \equiv 0 \quad\left(\bmod \varpi, \varpi_{1}, \ldots, \varpi_{s}\right), \\
X\rfloor d \varpi_{a} \equiv-\left(C_{a}^{i \beta \gamma} \varpi_{\beta \gamma}+D_{a b}^{i} \omega^{b}\right)(X) \varpi_{i}-\left(C_{a}^{\alpha \beta \gamma} \varpi_{\beta \gamma}+D_{a b}^{\alpha} \omega^{b}\right)(X) \varpi_{\alpha} \quad\left(\bmod \varpi, \varpi_{1}, \ldots, \varpi_{s}\right)
\end{array}\right.
$$

Thus $X \in \operatorname{Ch}\left(\tilde{N}^{*}\right)(v)$ iff $\left(C_{a}^{i \beta \gamma} \varpi_{\beta \gamma}+D_{a b}^{i} \omega^{b}\right)(X)=\left(C_{a}^{\alpha \beta \gamma} \varpi_{\beta \gamma}+D_{a b}^{\alpha} \omega^{b}\right)(X)=0$, which completes the proof of $(3)$.

Thus we get

Lemma 5.4. (1) If $r<n-1$ and $\mathfrak{f}(E)$ is completely integrable, then $\mathfrak{f}(E)=C h\left(D^{1}\right) \cap C h(\tilde{N})$. (2) If $C(E)$ is completely integrable, then $C_{a}^{\alpha \beta \gamma}=C_{a}^{i \beta \gamma}=D_{a b}^{\alpha}=D_{i b}^{\alpha}=0$ and $D_{a b}^{i}=D_{b a}^{i}$. Moreover $C_{i}^{\alpha \beta \gamma}=0$, when $r<n-1$. In particular, if $r<n-1$ and $C(E)$ is completely integrable, then $C(E)=C h(\tilde{N})$.

Proof. (1) If $\mathfrak{f}(E)$ is completely integrable, we have

$$
d \omega^{i} \equiv d \omega^{\alpha} \equiv d \pi_{\lambda} \equiv 0 \quad\left(\bmod \varpi, \varpi_{1}, \ldots, \varpi_{n}, \omega^{1}, \ldots, \omega^{n}, \pi_{1}, \ldots, \pi_{t}\right) .
$$

Then, if $r<n-1, C_{a}^{\alpha \beta \gamma}=0$ follows from (5.2) and $C_{i}^{\alpha \beta \gamma}=0$ follows from (5.3), which proves (1) by (1) in Lemma 5.3.

(2) If $C(E)$ is completely integrable, we have

$$
d \omega^{i} \equiv d \omega^{\alpha} \equiv d \pi_{\lambda} \equiv 0 \quad\left(\bmod \varpi, \varpi_{1}, \ldots, \varpi_{n}, \omega^{s+1}, \ldots, \omega^{n}, \pi_{1}, \ldots, \pi_{t}\right) .
$$

Then $C_{a}^{\alpha \beta \gamma}=0$ follows from (5.1), $D_{a b}^{\alpha}=0$ follows from (5.2), $D_{i b}^{\alpha}=0$ follows from (5.3), $C_{a}^{i \beta \gamma}=0$ and $D_{a b}^{i}=D_{b a}^{i}$ follows from (5.4) respectively. Moreover, when $r<n-1, C_{i}^{\alpha \beta \gamma}=0$ follows from (5.3). Then the last assertion follows from (2) in Lemma 5.3.

Moreover we have

Lemma 5.5. In case $r=n-1$, if $C(E)$ is completely integrable and rank $C h\left(D^{2}\right)<\operatorname{dim} \hat{E}$, then $C(E)=C h(\tilde{N})$.

Proof. We assume that $r=n-1$ and $C(E)$ is completely integrable. Then, by (2) in Lemma 5.4, we have $C_{a}^{n n n}=C_{a}^{i n n}=D_{a b}^{n}=D_{i b}^{n}=0$ and $D_{a b}^{i}=D_{b a}^{i}$. Thus, by (2) in Lemma 5.3, it suffices to 
show $C_{i}^{n n n}=0$ when $\operatorname{rank} \operatorname{Ch}\left(D^{2}\right)<\operatorname{dim} \hat{E}$. Putting $\mathfrak{M}_{2}=\left\langle\left\{\varpi, \varpi_{1}, \ldots, \varpi_{n}, \omega^{1}, \ldots, \omega^{s}, \omega^{n}\right\}\right\rangle$, we have

$$
d \varpi \equiv d \varpi_{a} \equiv 0, \quad d \varpi_{i} \equiv \omega^{j} \wedge \pi_{i j}, \quad d \varpi_{n} \equiv \omega^{i} \wedge \pi_{i n}, \quad\left(\bmod \quad \mathfrak{M}_{2}\right) .
$$

Then, taking exterior derivatives of both sides of (B.3) and calculating mod $\mathfrak{M}_{2}$, we get

$$
d \omega^{n} \wedge \pi_{a n}+d \varpi_{j} \wedge\left(B_{a}^{j \lambda} \pi_{\lambda}+E_{a k}^{j} \omega^{k}\right)+d \varpi_{n} \wedge\left(B_{a}^{n \lambda} \pi_{\lambda}+E_{a j}^{n} \omega^{j}\right) \equiv 0 \quad\left(\bmod \quad \mathfrak{M}_{2}\right) .
$$

From (5.1), we have $d \omega^{n} \equiv\left(B_{i}^{n \lambda} \pi_{\lambda}+C_{i}^{n n n} \varpi_{n n}+E_{i j}^{n} \omega^{j}\right) \wedge \omega^{i}\left(\bmod \mathfrak{M}_{2}\right)$. Hence we get

$$
\begin{aligned}
\left(B_{i}^{n \lambda} \pi_{\lambda}+C_{i}^{n n n} \varpi_{n n}+E_{i j}^{n} \omega^{j}\right) \wedge \omega^{i} \wedge \pi_{a n}+\omega^{j} \wedge \pi_{i j} \wedge\left(B_{a}^{j \lambda} \pi_{\lambda}+E_{a k}^{j} \omega^{k}\right) & \\
& +\omega^{i} \wedge \pi_{i n} \wedge\left(B_{a}^{n \lambda} \pi_{\lambda}+E_{a j}^{n} \omega^{j}\right) \equiv 0 \quad\left(\bmod \quad \mathfrak{M}_{2}\right) .
\end{aligned}
$$

Then, since $\pi_{a n}, \pi_{i j}, \pi_{i n}$ are linear combinations of $\left\{\pi_{\lambda}(1 \leqq \lambda \leqq t)\right\}\left(\bmod \varpi, \varpi_{1}, \ldots, \varpi_{n}\right)$, we obtain $C_{i}^{n n n} \varpi_{n n} \wedge \omega^{i} \wedge \pi_{a n}=0$. By the assumption rank $\operatorname{Ch}\left(D^{2}\right)<\operatorname{dim} \hat{E}$, we have $\pi_{a n} \neq 0$ for some $a$. Hence we get $C_{i}^{n n n} \varpi_{n n} \wedge \omega^{i}=0$, which implies $C_{i}^{n n n}=0$. This completes the proof.

Finally we add the following.

Lemma 5.6. If $C(E)=C h(\tilde{N})$ and

$\left\langle\left\{\pi_{i j}(s+1 \leqq i, j \leqq r+1)\right\}\right\rangle \cap\left\langle\left\{\pi_{a \alpha}(1 \leqq a \leqq s, r+1 \leqq \alpha \leqq n)\right\}\right\rangle=\{0\}$, then $C(E) \subset C h\left(\tilde{N}^{*}\right)$.

Proof. From $C(E)=\operatorname{Ch}(\tilde{N})$ and by (2) in Lemma 5.3, we have $C_{a}^{\alpha \beta \gamma}=C_{i}^{\alpha \beta \gamma}=D_{a b}^{\alpha}=D_{i b}^{\alpha}=0$. Moreover, by (2) in Lemma 5.4, we have $C_{a}^{i \beta \gamma}=0$. Hence, by (3) in Lemma 5.3, it suffices to show $D_{a b}^{i}=0$, under the above condition. Putting $\mathfrak{M}_{3}=\left\langle\left\{\varpi, \varpi_{1}, \ldots, \varpi_{n}, \omega^{r+1}, \ldots, \omega^{n}\right\}\right\rangle$, we have

$$
d \varpi \equiv d \varpi_{a} \equiv 0, \quad d \varpi_{i} \equiv \omega^{j} \wedge \pi_{i j}, \quad d \varpi_{\alpha} \equiv \omega^{b} \wedge \pi_{b \alpha}+\omega^{i} \wedge \pi_{i \alpha}, \quad\left(\bmod \quad \mathfrak{M}_{3}\right) .
$$

Then, taking exterior derivatives of both sides of (B.3) and calculating mod $\mathfrak{M}_{3}$, we get

$$
d \omega^{\alpha} \wedge \pi_{a \alpha}+d \varpi_{j} \wedge\left(B_{a}^{j \lambda} \pi_{\lambda}+D_{a b}^{j} \omega^{b}+E_{a k}^{j} \omega^{k}\right)+d \varpi_{\alpha} \wedge\left(B_{a}^{\alpha \lambda} \pi_{\lambda}+E_{a j}^{\alpha} \omega^{j}\right) \equiv 0 \quad\left(\bmod \quad \mathfrak{M}_{3}\right) .
$$

From (5.1), we have $d \omega^{\alpha} \equiv\left(B_{b}^{\alpha \lambda} \pi_{\lambda}+E_{b j}^{\alpha} \omega^{j}\right) \wedge \omega^{b}+\left(B_{i}^{\alpha \lambda} \pi_{\lambda}+E_{i j}^{\alpha} \omega^{j}\right) \wedge \omega^{i}\left(\bmod \mathfrak{M}_{3}\right)$. Hence we get

$$
\begin{aligned}
\left(B_{b}^{\alpha \lambda} \pi_{\lambda}+E_{b j}^{\alpha} \omega^{j}\right) \wedge \omega^{b} \wedge \pi_{a \alpha}+ & \left(B_{i}^{\alpha \lambda} \pi_{\lambda}+E_{i j}^{\alpha} \omega^{j}\right) \wedge \omega^{i} \wedge \pi_{a \alpha}+\omega^{k} \wedge \pi_{j k} \wedge\left(B_{a}^{j \lambda} \pi_{\lambda}+D_{a b}^{j} \omega^{b}+E_{a i}^{j} \omega^{i}\right) \\
& +\left(\omega^{b} \wedge \pi_{b \alpha}+\omega^{i} \wedge \pi_{i \alpha}\right) \wedge\left(B_{a}^{\alpha \lambda} \pi_{\lambda}+E_{a j}^{\alpha} \omega^{j}\right) \equiv 0 \quad\left(\bmod \quad \mathfrak{M}_{3}\right) .
\end{aligned}
$$

Then, since $\pi_{a \alpha}, \pi_{i j}, \pi_{i \alpha}$ are linear combinations of $\left\{\pi_{\lambda}(1 \leqq \lambda \leqq t)\right\}\left(\bmod \varpi, \varpi_{1}, \ldots, \varpi_{n}\right)$, we have

$$
\omega^{b} \wedge \omega^{j} \wedge\left(E_{b j}^{\alpha} \pi_{a \alpha}+E_{a j}^{\alpha} \pi_{b \alpha}-D_{a b}^{i} \pi_{i j}\right) \equiv 0 \quad\left(\bmod \quad \mathfrak{M}_{3}\right) .
$$

Then, by the assumption $\left\langle\left\{\pi_{i j}(s+1 \leqq i, j \leqq r+1)\right\}\right\rangle \cap\left\langle\left\{\pi_{a \alpha}(1 \leqq a \leqq s, r+1 \leqq \alpha \leqq n)\right\}\right\rangle=\{0\}$, we get $D_{a b}^{i} \omega^{b} \wedge \omega^{j} \wedge \pi_{i j} \equiv 0\left(\bmod \mathfrak{M}_{3}\right)$. Thus we obtain

$$
D_{a b}^{i} \omega^{j} \wedge \pi_{i j}=D_{a b}^{i} \Omega_{i} \equiv 0 \quad\left(\bmod \varpi, \varpi_{1}, \ldots, \varpi_{n}, \omega^{a}, \omega^{\alpha}(1 \leqq a \leqq s, r+1 \leqq \alpha \leqq n)\right) .
$$

Since $\left\{\Omega_{s+1}, \ldots, \Omega_{r}\right\}$ are linearly independent $\left(\bmod \varpi, \varpi_{1}, \ldots, \varpi_{n}\right)$, we obtain $D_{a b}^{i}=0$, which completes the proof.

Summarizing the discussion above, we obtain the first part of Second Reduction Theorem for $P D$ manifolds of second order, which are regular of type $\mathfrak{s}$. 
Theorem 5.1. Let $\left(R ; D^{1}, D^{2}\right)$ be a PD manifold of second order, which is regular of type $\mathfrak{s}$. Assume that there exists $G_{0}(\mathfrak{s})$-invariant subspace $E$ of $V$ of dimension $r$ such that $\mathfrak{f}^{\perp} \subset E \otimes_{S} V$.

(1) In case $r<n-1$, if $\mathfrak{f}(E)$ is completely integrable, then $\mathfrak{f}(E)=C h\left(D^{1}\right) \cap C h(\tilde{N})$ and $\kappa_{1}:\left(R ; D^{1}, D^{2}\right) \rightarrow\left(R(W) ; D_{W}^{1}, D_{W}^{2}\right)$ is a local isomorphism.

(2) In case $r<n-1$, if $C(E)$ is completely integrable, then $C(E)=C h(\tilde{N})$ and $\kappa_{1}$ : $\left(R ; D^{1}, D^{2}\right) \rightarrow\left(R(W) ; D_{W}^{1}, D_{W}^{2}\right)$ is a local isomorphism.

(3) In case $r=n-1$, further assume that $\operatorname{rank} C h\left(D^{2}\right)<\operatorname{dim} \hat{E}$, if $C(E)$ is completely integrable, then $C(E)=C h(\tilde{N})$ and $\kappa_{1}:\left(R ; D^{1}, D^{2}\right) \rightarrow\left(R(W) ; D_{W}^{1}, D_{W}^{2}\right)$ is a local isomorphism.

Thus in these cases, the equivalence of $P D$ manifolds of second order $\left(R ; D^{1}, D^{2}\right)$, which are regular of type $\mathfrak{s}$, is reducible to that of the associated $I G$ manifolds of corank $r\left(W ; C^{*}, N\right)$, as in $§ 5.1$.

5.3. Two Step Reduction. Let $\left(R ; D^{1}, D^{2}\right)$ be a $P D$ manifold of second order, which is regular of type $\mathfrak{s}$. We assume that there exists $G_{0}(\mathfrak{s})$-invariant subspace $E$ of $V$ of dimension $r$ such that $\mathfrak{f}^{\perp} \subset E \otimes_{S} V$. When $\hat{E} \neq\{0\}$, we can discuss the further reduction procedure as in the following.

We assume that $C(E)=\operatorname{Ch}(\tilde{N})$. If $\hat{E} \neq\{0\}$, since $\tilde{N}=\eta_{*}^{-1}(N), N$ has non-trivial Cauchy characteristic system $\mathrm{Ch}(N)$ on $W$ such that $\operatorname{rank} \operatorname{Ch}(N)=\operatorname{dim} \hat{E}$. Now we assume that $W$ is regular with respect to $\mathrm{Ch}(N)$, i.e., the space $Y=W / \mathrm{Ch}(N)$ of leaves of this foliation is a manifold such that each fibre of the projection $\beta: W \rightarrow Y$ is connected and $\beta$ is a submersion. We further assume that $C(E) \subset \mathrm{Ch}\left(\tilde{N}^{*}\right)$. Then $\mathrm{Ch}(N) \subset \mathrm{Ch}\left(N^{*}\right)$ on $W$. Moreover, by Lemma $5.2(3), \mathrm{Ch}\left(N^{*}\right) \subset N$ on $W$. Hence there exist differential systems $D_{N}^{*}$ and $D_{N}$ on $Y$ of codimension $s+1$ and $r+1$ respectively such that $N^{*}=\beta_{*}^{-1}\left(D_{N}^{*}\right), N=\beta_{*}^{-1}\left(D_{N}\right)$, $D_{N} \supset \mathrm{Ch}\left(D_{N}^{*}\right)$ and $\mathrm{Ch}\left(D_{N}\right)$ is trivial. In this situation, from $\left(Y ; D_{N}^{*}, D_{N}\right)$, we can reconstruct the $I G$ manifold $\left(W ; C^{*}, N\right)$, at least locally, as follows. First let us consider the collection $\tilde{W}(Y)$ of hyperplanes $w$ in each tangent space $T_{y}(Y)$ at $y \in Y$ which contains the fibre $D_{N}^{*}(y)$ of $D_{N}^{*}$.

$$
\begin{gathered}
\tilde{W}(Y)=\bigcup_{y \in Y} \tilde{W}_{y} \subset J(Y, m-1), \\
\tilde{W}_{y}=\left\{w \in G r\left(T_{y}(Y), m-1\right) \mid w \supset D_{N}^{*}(y)\right\} \cong P\left(T_{y}(Y) / D_{N}^{*}(y)\right)=\mathbb{P}^{s},
\end{gathered}
$$

where $m=\operatorname{dim} Y$ and $s=\operatorname{dim} \hat{E}$. Moreover $C_{Y}^{*}$ is the canonical system obtained by the Grassmannian construction and $N_{Y}^{*}, N_{Y}$ are the lifts of $D_{N}^{*}, D_{N}$. Precisely we have

$$
C_{Y}^{*}(w)=\mu_{*}^{-1}(w) \supset N_{Y}^{*}(w)=\mu_{*}^{-1}\left(D_{N}^{*}(y)\right) \supset N_{Y}(w)=\mu_{*}^{-1}\left(D_{N}(y)\right),
$$

for each $w \in \tilde{W}(Y)$ and $y=\mu(w)$, where $\mu: \tilde{W}(Y) \rightarrow Y$ is the projection. Then we have a map $\kappa_{2}$ of $W$ into $\tilde{W}(Y)$ given by

$$
\kappa_{2}(w)=\beta_{*}\left(C^{*}(w)\right) \subset T_{y}(Y),
$$

for each $w \in W$ and $y=\beta(w)$. By Realization Lemma for $\left(W, C^{*}, \beta, Y\right), \kappa_{2}$ is a map of constant rank such that

$$
\operatorname{Ker}\left(\kappa_{2}\right)_{*}=\operatorname{Ch}\left(C^{*}\right) \cap \operatorname{Ker} \beta_{*}=\operatorname{Ch}\left(C^{*}\right) \cap \operatorname{Ch}(N)=\{0\} .
$$

Thus $\kappa_{2}$ is an immersion and, by a dimension count, in fact, a local diffeomorphism of $W$ into $\tilde{W}(Y)$ such that

$$
\left(\kappa_{2}\right)_{*}\left(C^{*}\right)=C_{Y}^{*}, \quad\left(\kappa_{2}\right)_{*}\left(N^{*}\right)=N_{Y}^{*}, \quad \text { and } \quad\left(\kappa_{2}\right)_{*}(N)=N_{Y} .
$$

Namely $\kappa_{2}:\left(W ; C^{*}, N\right) \rightarrow\left(\tilde{W}(Y) ; C_{Y}^{*}, N_{Y}\right)$ is a local isomorphism of $I G$ manifold of corank $r$. Thus $\left(W ; C^{*}, N\right)$ is reconstructed from $\left(Y ; D_{N}^{*}, D_{N}\right)$, at least locally, as a part of $\left(\tilde{W}(Y) ; C_{Y}^{*}, N_{Y}\right)$. 
By the construction of $\left(\tilde{W}(Y) ; C_{Y}^{*}, N_{Y}\right)$, an isomorphism of $\left(Y ; D_{N}^{*}, D_{N}\right)$ naturally lifts to an isomorphism of $\left(\tilde{W}(Y) ; C_{Y}^{*}, N_{Y}\right)$.

Summarizing the above discussion, we obtain the following Second Reduction Theorem (Two Step Reduction Theorem) for $P D$ manifolds of second order, which are regular of type $\mathfrak{s}$ such that there exists a $G_{0}(\mathfrak{s})$-invariant subspace $E$ of $V$ of dimension $r$ satisfying $\mathfrak{f}^{\perp} \subset E \otimes_{S} V$ and $\operatorname{dim} \hat{E}=s>0$.

Theorem 5.2. Let $\left(R ; D^{1}, D^{2}\right)$ and $\left(\hat{R} ; \hat{D}^{1}, \hat{D}^{2}\right)$ be PD manifolds of second order, which are regular of type $\mathfrak{s}$. Assume that there exists a $G_{0}(\mathfrak{s})$-invariant subspace $E$ of $V$ of dimension $r$ satisfying $\mathfrak{f}^{\perp} \subset E \otimes_{S} V$ and $\operatorname{dim} \hat{E}=s>0$. Moreover assume the following two conditions for the covariant sytems of each $P D$ manifold;

(i) $C(E)$ and $\hat{C}(E)$ are completely integrable (when $r=n-1$, assume further rank $C h\left(D^{2}\right)<$ $s$ and $\left.\operatorname{rank} C h\left(\hat{D}^{2}\right)<s\right)$.

(ii) $C(E) \subset C h\left(\tilde{N}^{*}\right)$ and $\hat{C}(E) \subset C h\left(\hat{N}^{*}\right)$.

Let $\left(W ; C^{*}, N\right)$ and $\left(\hat{W} ; \hat{C}^{*}, \hat{N}\right)$ be the associated $I G$ manifolds of corank $r$ of $\left(R ; D^{1}, D^{2}\right)$ and $\left(\hat{R} ; \hat{D}^{1}, \hat{D}^{2}\right)$ respectively. Assume that $W$ and $\hat{W}$ are regular with respect to $C h(N)$ and $C h(\hat{N})$ respectively. Let $\left(Y ; D_{N}^{*}, D_{N}\right)$ and $\left(\hat{Y}, D_{\hat{N}}^{*}, D_{\hat{N}}\right)$ be the leaf spaces, where $Y=W / C h(N)$ and $\hat{Y}=\hat{W} / C h(\hat{N})$. Let us fix points $v_{o} \in R$ and $\hat{v}_{o} \in \hat{R}$ and put $w_{o}=\eta\left(v_{o}\right), y_{o}=\beta\left(w_{o}\right)$ and $\hat{w}_{o}=\hat{\eta}\left(\hat{v}_{o}\right), \hat{y}_{o}=\hat{\beta}\left(\hat{w}_{o}\right)$. Then a local isomorphism $\psi:\left(R ; D^{1}, D^{2}\right) \rightarrow\left(\hat{R} ; \hat{D}^{1}, \hat{D}^{2}\right)$ such that $\psi\left(v_{o}\right)=\hat{v}_{o}$ induces a local isomorphism $\varphi:\left(W ; C^{*}, N\right) \rightarrow\left(\hat{W}, \hat{C}^{*}, \hat{N}\right)$ such that $\varphi\left(w_{o}\right)=\hat{w}_{o}$ and $\varphi_{*}\left(\kappa_{1}\left(v_{o}\right)\right)=\hat{\kappa}_{1}\left(\hat{v}_{o}\right)$, and vice versa. Furthermore a local isomorphism $\varphi:\left(W ; C^{*}, N\right) \rightarrow$ $\left(\hat{W}, \hat{C}^{*}, \hat{N}\right)$ such that $\varphi\left(w_{o}\right)=\hat{w}_{o}$ induces a local isomorphism $\phi:\left(Y ; D_{N}^{*}, D_{N}\right) \rightarrow\left(\hat{Y} ; D_{\hat{N}}^{*}, D_{\hat{N}}\right)$ such that $\phi\left(y_{o}\right)=\hat{y}_{o}$ and $\phi_{*}\left(\kappa_{2}\left(w_{o}\right)\right)=\hat{\kappa}_{2}\left(\hat{w}_{o}\right)$, and vice versa.

Here we remark that, when $\hat{E}$ coincides with $E$, i.e., when $s=r$, we have $N^{*}=N$ and $D_{N}^{*}=D_{N}$. Hence, in this case, the condition $(i i)$ is automatically satisfied under the condition (i) and the equivalence of $\left(R ; D^{1}, D^{2}\right)$ is reducible to that of $\left(Y, D_{N}\right)$.

We will discuss conditions for the symbol algebra $\mathfrak{s}$, where the condition $(i)$ or $(i i)$ in the above Theorem is automatically satisfied, in the next section.

\section{Typical Classes and their Generalizations}

6.1. Minimum subspace $F$ satisfying $\mathfrak{f}^{\perp} \subset S^{2}(F)$. Let $\left(R ; D^{1}, D^{2}\right)$ be a $P D$ manifold of second order, which is regular of type $\mathfrak{s}$. For the symbol algebra $\mathfrak{s}=\mathfrak{s}_{-3} \oplus \mathfrak{s}_{-2} \oplus \mathfrak{s}_{-1}$, where

$$
\mathfrak{s}_{-3}=\mathbb{R}, \quad \mathfrak{s}_{-2}=V^{*}, \quad \mathfrak{s}_{-1}=V \oplus \mathfrak{f}, \quad \mathfrak{f} \subset S^{2}\left(V^{*}\right),
$$

there exists a unique minimum subspace $F$ of $V$ such that $\mathfrak{f}^{\perp} \subset S^{2}(F) \subset S^{2}(V)$, under the identification: $S^{2}(V) \cong\left(S^{2}\left(V^{*}\right)\right)^{*}$. This follows from

$$
S^{2}\left(F_{1}\right) \cap S^{2}\left(F_{2}\right)=S^{2}\left(F_{1} \cap F_{2}\right),
$$

for $F_{1}$ and $F_{2}$ such that $\mathfrak{f}^{\perp} \subset S^{2}\left(F_{i}\right)(i=1,2) . F$ may coincide with $V$. Moreover, it follows from $S^{2}(a(F))=a\left(S^{2}(F)\right) \supset a\left(\mathfrak{f}^{\perp}\right)=\mathfrak{f}^{\perp}$ for $a \in G_{0}(\mathfrak{s})$ that $F$ is $G_{0}(\mathfrak{s})$-invariant. We put

$$
\hat{F}=\left\{v \in F \mid v \odot F \subset \mathfrak{f}^{\perp}\right\} .
$$

Let $r$ and $s$ be the dimensions of $F$ and $\hat{F}$ respectively. In the rest of this section, we assume that $\{0\} \varsubsetneqq F \varsubsetneqq V$.

Now we will discuss the Second Reduction Procedure for $\left(R ; D^{1}, D^{2}\right)$, utilizing the minimum subspace $F$ satisfying $\mathfrak{f}^{\perp} \subset S^{2}(F)\left(\subset F \otimes_{S} V\right)$. Let us fix a point $v \in R$. Then, as in $\S 5.2$, there exists a coframe $\left\{\varpi, \varpi_{1}, \ldots, \varpi_{n}, \omega^{1}, \ldots, \omega^{n}, \varpi_{k \beta}(1 \leqq k \leqq r, r+1 \leqq \beta \leqq n), \varpi_{\alpha \beta}(r+1 \leqq\right.$ 
$\left.\alpha \leqq \beta \leqq n), \pi_{\lambda}\left(1 \leqq \lambda \leqq t_{1}\right)\right\}\left(t_{1}=\operatorname{dim} \mathfrak{f}-\operatorname{dim} F^{\perp} \otimes_{S} V^{*}\right)$ and 1 -forms $\left\{\pi_{i j}(s+1 \leqq i, j \leqq r)\right\}$ defined around $v \in R$ such that

$D^{1}=\{\varpi=0\}, \quad D^{2}=\left\{\varpi=\varpi_{1}=\cdots=\varpi_{n}=0\right\}, \quad \tilde{N}=\tilde{N}(F)=\left\{\varpi=\varpi_{1}=\cdots=\varpi_{r}=0\right\}$,

$\tilde{N}^{*}=\tilde{N}(\hat{F})=\left\{\varpi=\varpi_{1}=\cdots=\varpi_{s}=0\right\}, \quad \tilde{N}^{\perp}=\left\{\varpi=\varpi_{1}=\cdots=\varpi_{n}=\omega^{r+1}=\cdots=\omega^{n}=0\right\}$,

and the following structure equations hold;

$$
d \varpi \equiv \omega^{1} \wedge \varpi_{1}+\cdots+\omega^{n} \wedge \varpi_{n} \quad(\bmod \varpi),
$$

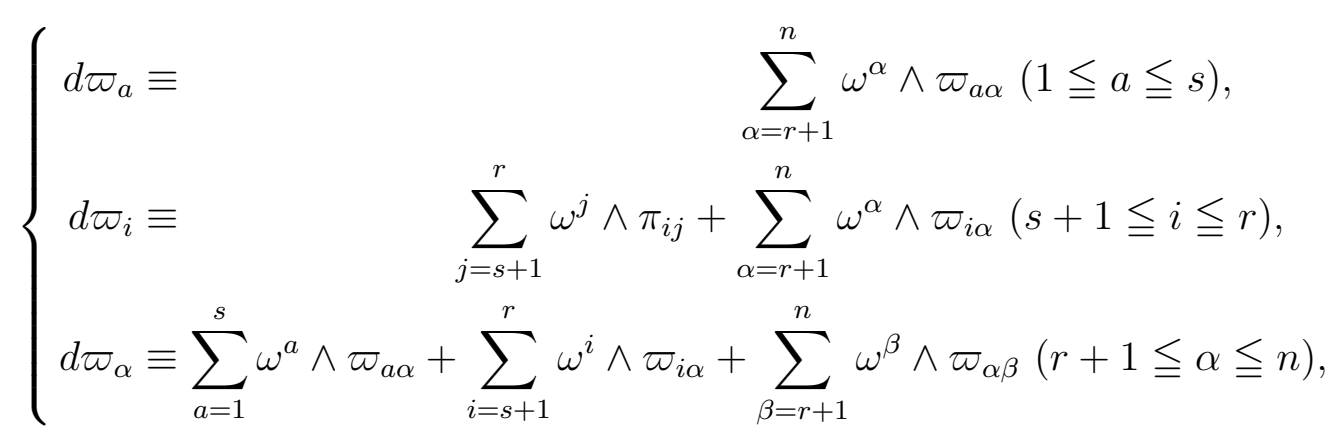

$\left(\bmod \varpi, \varpi_{1}, \ldots, \varpi_{n}\right)$, where $\varpi_{\alpha \beta}=\varpi_{\beta \alpha}, \pi_{i j}=\pi_{j i}$, and $\left\{\pi_{\lambda}\left(1 \leqq \lambda \leqq t_{1}\right)\right\}$ is a basis of $\left\langle\left\{\pi_{i j}(s+\right.\right.$ $1 \leqq i, j \leqq r)\}\rangle\left(\bmod \varpi, \varpi_{1}, \ldots, \varpi_{n}\right)$. Moreover $\left\{\Omega_{s+1}, \ldots, \Omega_{r}\right\}$ are linearly independent $(\bmod$ $\left.\varpi, \varpi_{1} \ldots, \varpi_{n}\right)$, where $\Omega_{i}=\sum_{j=s+1}^{r} \omega^{j} \wedge \pi_{i j}$. Furthermore, we have

$$
\begin{array}{r}
C(F)=\left\{\varpi=\varpi_{1}=\cdots=\varpi_{n}=\omega^{s+1}=\cdots=\omega^{n}=\pi_{\lambda}=\varpi_{k \alpha}=0\right. \\
\left.\quad\left(1 \leqq \lambda \leqq t_{1}, 1 \leqq k \leqq r, r+1 \leqq \alpha \leqq n\right)\right\},
\end{array}
$$

and

$$
\begin{aligned}
\mathfrak{f}(F)=\left\{\varpi=\varpi_{1}=\cdots=\varpi_{n}=\omega^{1}=\cdots=\omega^{n}=\pi_{\lambda}=\varpi_{k \alpha}=0\right. & \\
& \left.\left(1 \leqq \lambda \leqq t_{1}, 1 \leqq k \leqq r, r+1 \leqq \alpha \leqq n\right)\right\} .
\end{aligned}
$$

In the rest of this section, we will adopt the Einstein's convention for indices. The index ranges are as follows; $1 \leqq a, b \leqq s, s+1 \leqq i, j, k \leqq r, r+1 \leqq \alpha, \beta \leqq n$ and $1 \leqq \lambda \leqq t_{1}$. From ( $\left.B^{*} .1\right)$, we have

$$
\left\{\begin{array}{rlrl}
d \varpi_{a} \equiv & \omega^{\alpha} & \wedge \varpi_{a \alpha}+A_{a}^{\alpha \beta} \varpi_{\alpha} \wedge \varpi_{\beta}+B_{a}^{\alpha \lambda} \varpi_{\alpha} \wedge \pi_{\lambda}+B_{a}^{\alpha b \beta} \varpi_{\alpha} \wedge \varpi_{b \beta}+ \\
B_{a}^{\alpha i \beta} \varpi_{\alpha} \wedge \varpi_{i \beta} & +C_{a}^{\alpha \beta \gamma} \varpi_{\alpha} \wedge \varpi_{\beta \gamma}+D_{a b}^{\alpha} \varpi_{\alpha} \wedge \omega^{b}+E_{a i}^{\alpha} \varpi_{\alpha} \wedge \omega^{i}+F_{a \beta}^{\alpha} \varpi_{\alpha} \wedge \omega^{\beta}, \\
d \varpi_{i} \equiv \omega^{j} \wedge \pi_{i j}+ & \omega^{\alpha} \wedge \varpi_{i \alpha}+A_{i}^{\alpha \beta} \varpi_{\alpha} \wedge \varpi_{\beta}+B_{i}^{\alpha \lambda} \varpi_{\alpha} \wedge \pi_{\lambda}+B_{i}^{\alpha b \beta} \varpi_{\alpha} \wedge \varpi_{b \beta}+ \\
B_{i}^{\alpha j \beta} \varpi_{\alpha} \wedge \varpi_{j \beta} & & +C_{i}^{\alpha \beta \gamma} \varpi_{\alpha} \wedge \varpi_{\beta \gamma}+D_{i b}^{\alpha} \varpi_{\alpha} \wedge \omega^{b}+E_{i j}^{\alpha} \varpi_{\alpha} \wedge \omega^{j}+F_{i \beta}^{\alpha} \varpi_{\alpha} \wedge \omega^{\beta},
\end{array}\right.
$$

$\left(\bmod \varpi, \varpi_{1}, \ldots, \varpi_{r}\right)$. First, replacing $\varpi_{a \alpha}$ and $\varpi_{i \alpha}$ by $\varpi_{a \alpha}-F_{a \alpha}^{\beta} \varpi_{\beta}$ and $\varpi_{i \alpha}-F_{i \alpha}^{\beta} \varpi_{\beta}$ respectively, we may assume that $F_{a \alpha}^{\beta}=F_{i \alpha}^{\beta}=0$. From $\left(A^{*}\right)$, we have $d \varpi \equiv 0\left(\bmod \mathfrak{M}_{1}^{*}\right)$, where $\mathfrak{M}_{1}^{*}=\left\langle\left\{\varpi, \varpi_{1}, \ldots, \varpi_{r}, \omega^{r+1}, \ldots, \omega^{n}\right\}\right\rangle$. Hence, from $\left(B^{*} .2\right)$, we have

$$
\begin{array}{rr}
d \omega^{\alpha} \wedge \varpi_{\alpha} \equiv \omega^{b} \wedge d \varpi_{b}+\omega^{i} \wedge d \varpi_{i} & \\
\equiv \omega^{b} \wedge\left(A_{b}^{\alpha \beta} \varpi_{\alpha} \wedge \varpi_{\beta} \quad+B_{b}^{\alpha \lambda} \varpi_{\alpha} \wedge \pi_{\lambda}+B_{b}^{\alpha c \beta} \varpi_{\alpha} \wedge \varpi_{c \beta}+B_{b}^{\alpha i \beta} \varpi_{\alpha} \wedge \varpi_{i \beta}\right. \\
\left.+C_{b}^{\alpha \beta \gamma} \varpi_{\alpha} \wedge \varpi_{\beta \gamma}+D_{b c}^{\alpha} \varpi_{\alpha} \wedge \omega^{c}+E_{b i}^{\alpha} \varpi_{\alpha} \wedge \omega^{i}\right) \\
+\omega^{i} \wedge\left(\omega^{j} \wedge \pi_{i j}+A_{i}^{\alpha \beta} \varpi_{\alpha} \wedge \varpi_{\beta} \quad+B_{i}^{\alpha \lambda} \varpi_{\alpha} \wedge \pi_{\lambda}+B_{i}^{\alpha b \beta} \varpi_{\alpha} \wedge \varpi_{b \beta}+B_{i}^{\alpha j \beta} \varpi_{\alpha} \wedge \varpi_{j \beta}\right. \\
\left.+C_{i}^{\alpha \beta \gamma} \varpi_{\alpha} \wedge \varpi_{\beta \gamma}+D_{i b}^{\alpha} \varpi_{\alpha} \wedge \omega^{b}+E_{i j}^{\alpha} \varpi_{\alpha} \wedge \omega^{j}\right)
\end{array}
$$


$\left(\bmod \mathfrak{M}_{1}^{*}\right)$. Thus we get

$$
\begin{aligned}
d \omega^{\alpha} \equiv & -\omega^{b} \wedge\left(B_{b}^{\alpha \lambda} \pi_{\lambda}+B_{b}^{\alpha c \beta} \varpi_{c \beta}+B_{b}^{\alpha i \beta} \varpi_{i \beta}+C_{b}^{\alpha \beta \gamma} \varpi_{\beta \gamma}+D_{b c}^{\alpha} \omega^{c}+E_{b i}^{\alpha} \omega^{i}\right) \\
& -\omega^{i} \wedge\left(B_{i}^{\alpha \lambda} \pi_{\lambda}+B_{i}^{\alpha b \beta} \varpi_{b \beta}+B_{i}^{\alpha j \beta} \varpi_{j \beta}+C_{i}^{\alpha \beta \gamma} \varpi_{\beta \gamma}+D_{i b}^{\alpha} \omega^{b}+E_{i j}^{\alpha} \omega^{j}\right)
\end{aligned}
$$

$\left(\bmod \varpi, \varpi_{1}, \ldots, \varpi_{n}, \omega^{r+1}, \ldots, \omega^{n}\right)$. Putting $\mathfrak{M}_{2}^{*}=\left\langle\left\{\varpi, \varpi_{1}, \ldots, \varpi_{n}, \omega^{\alpha}, \pi_{\lambda}(r+1 \leqq \alpha \leqq n, 1 \leqq\right.\right.$ $\left.\left.\left.\lambda \leqq t_{1}\right)\right\}\right\rangle$, we have

$$
d \varpi \equiv d \varpi_{a} \equiv d \varpi_{i} \equiv 0, \quad d \varpi_{\alpha} \equiv \omega^{b} \wedge \varpi_{b \alpha}+\omega^{j} \wedge \varpi_{j \alpha} \quad\left(\bmod \quad \mathfrak{M}_{2}^{*}\right) .
$$

Then, taking exterior derivatives of both sides of the first equation of $\left(B^{*} .2\right)$ and calculating $\bmod \mathfrak{M}_{2}^{*}$, we get

$$
d \omega^{\alpha} \wedge \varpi_{a \alpha}+d \varpi_{\alpha} \wedge\left(B_{a}^{\alpha c \beta} \varpi_{c \beta}+B_{a}^{\alpha i \beta} \varpi_{i \beta}+C_{a}^{\alpha \beta \gamma} \varpi_{\beta \gamma}+D_{a c}^{\alpha} \omega^{c}+E_{a i}^{\alpha} \omega^{i}\right) \equiv 0 \quad\left(\bmod \quad \mathfrak{M}_{2}^{*}\right) .
$$

Substituting (6.1) into the above equation, we get

$$
\begin{aligned}
& \omega^{b} \wedge \varpi_{a \alpha} \wedge\left(B_{b}^{\alpha c \beta} \varpi_{c \beta}+B_{b}^{\alpha i \beta} \varpi_{i \beta}+C_{b}^{\alpha \beta \gamma} \varpi_{\beta \gamma}+D_{b c}^{\alpha} \omega^{c}+E_{b i}^{\alpha} \omega^{i}\right) \\
&+\omega^{i} \wedge \varpi_{a \alpha} \wedge\left(B_{i}^{\alpha c \beta} \varpi_{c \beta}+B_{i}^{\alpha j \beta} \varpi_{j \beta}+C_{i}^{\alpha \beta \gamma} \varpi_{\beta \gamma}+D_{i b}^{\alpha} \omega^{b}+E_{i j}^{\alpha} \omega^{j}\right) \\
&+\omega^{b} \wedge \varpi_{b \alpha} \wedge\left(B_{a}^{\alpha c \beta} \varpi_{c \beta}+B_{a}^{\alpha i \beta} \varpi_{i \beta}+C_{a}^{\alpha \beta \gamma} \varpi_{\beta \gamma}+D_{a c}^{\alpha} \omega^{c}+E_{a i}^{\alpha} \omega^{i}\right) \\
&+\omega^{j} \wedge \varpi_{j \alpha} \wedge\left(B_{a}^{\alpha c \beta} \varpi_{c \beta}+B_{a}^{\alpha i \beta} \varpi_{i \beta}+C_{a}^{\alpha \beta \gamma} \varpi_{\beta \gamma}+D_{a c}^{\alpha} \omega^{c}+E_{a i}^{\alpha} \omega^{i}\right) \equiv 0 \quad\left(\bmod \quad \mathfrak{M}_{2}^{*}\right) .
\end{aligned}
$$

Hence we obtain

$$
\begin{aligned}
& \omega^{i} \wedge \varpi_{a \alpha} \wedge\left(B_{i}^{\alpha c \beta} \varpi_{c \beta}+C_{i}^{\alpha \beta \gamma} \varpi_{\beta \gamma}+E_{i j}^{\alpha} \omega^{j}\right) \equiv 0 \quad\left(\bmod \quad \mathfrak{M}_{2}^{*}\right) . \\
& \omega^{b} \wedge \varpi_{a \alpha} \wedge\left(B_{b}^{\alpha c \beta} \varpi_{c \beta}+B_{b}^{\alpha i \beta} \varpi_{i \beta}+C_{b}^{\alpha \beta \gamma} \varpi_{\beta \gamma}+D_{b c}^{\alpha} \omega^{c}+\left(E_{b i}^{\alpha}-D_{i b}^{\alpha}\right) \omega^{i}\right) \\
& \quad+\omega^{b} \wedge \varpi_{b \alpha} \wedge\left(B_{a}^{\alpha c \beta} \varpi_{c \beta}+B_{a}^{\alpha i \beta} \varpi_{i \beta}+C_{a}^{\alpha \beta \gamma} \varpi_{\beta \gamma}+D_{a c}^{\alpha} \omega^{c}+E_{a i}^{\alpha} \omega^{i}\right) \equiv 0
\end{aligned}
$$

$\left(\bmod \mathfrak{M}_{2}^{*}\right)$.

$$
B_{i}^{\alpha j \beta} \omega^{i} \wedge \varpi_{a \alpha} \wedge \varpi_{j \beta}+B_{a}^{\alpha c \beta} \omega^{j} \wedge \varpi_{j \alpha} \wedge \varpi_{c \beta} \equiv 0 \quad\left(\bmod \quad \mathfrak{M}_{2}^{*}\right)
$$

$$
\omega^{j} \wedge \varpi_{j \alpha} \wedge\left(B_{a}^{\alpha i \beta} \varpi_{i \beta}+C_{a}^{\alpha \beta \gamma} \varpi_{\beta \gamma}+D_{a c}^{\alpha} \omega^{c}+E_{a i}^{\alpha} \omega^{i}\right) \equiv 0 \quad\left(\bmod \quad \mathfrak{M}_{2}^{*}\right) .
$$

From, (6.a.1), if $r-s>0$, we get $B_{i}^{\alpha c \beta}=0$ for $c \beta \neq a \alpha, C_{i}^{\alpha \beta \gamma}=0$ and $E_{i j}^{\alpha}=E_{j i}^{\alpha}$. In particular, when $r-s>0, B_{i}^{\alpha a \beta}=0$ if $s \geqq 2$ and $B_{i}^{\alpha 1 \beta}=B_{i}^{\beta 1 \alpha}$ if $s=1$. Moreover, by replacing $\pi_{i j}$ by $\pi_{i j}+E_{i j}^{\alpha} \varpi_{\alpha}$, we may assume $E_{i j}^{\alpha}=0$.

In case $s \geqq 2$, first let us choose any $a(1 \leqq a \leqq r)$. Since $s \geqq 2$, we can find $b$ such that $a \neq b$. Then, from (6.a.2), we see that the coefficients of $\omega^{b} \wedge \varpi_{b \alpha} \wedge \varpi_{i \beta}, \omega^{b} \wedge \varpi_{b \alpha} \wedge \varpi_{\beta \gamma}$, $\omega^{b} \wedge \varpi_{b \alpha} \wedge \omega^{i}$ and $\omega^{b} \wedge \varpi_{a \alpha} \wedge \omega^{i}$ are $B_{a}^{\alpha i \beta}, C_{a}^{\alpha \beta \gamma}, E_{a i}^{\alpha}$ and $\left(E_{b i}^{\alpha}-D_{i b}^{\alpha}\right)$, and those of $\omega^{b} \wedge \varpi_{b \alpha} \wedge \omega^{c}$ and $\omega^{a} \wedge \varpi_{a \alpha} \wedge \omega^{b}$ are $D_{a c}^{\alpha}$ and $2 D_{a b}^{\alpha}-D_{b a}^{\alpha}$ respectively. Hence we get $B_{a}^{\alpha i \beta}=0, C_{a}^{\alpha \beta \gamma}=0$, $E_{a i}^{\alpha}=0, D_{i a}^{\alpha}=0, D_{a c}^{\alpha}=0$ for $c \neq b$ and $2 D_{a b}^{\alpha}=D_{b a}^{\alpha}$. Similarly, interchanging the role of $a$ and $b$, from (6.b.2), we get $D_{b c}^{\alpha}=0$ for $c \neq a$ and $2 D_{b a}^{\alpha}=D_{a b}^{\alpha}$. Thus we obtain $D_{a c}^{\alpha}=0$ for any $c$. Moreover, from (6.a.2), we see that the coefficients of $\omega^{b} \wedge \varpi_{b \alpha} \wedge \varpi_{c \beta}(c \beta \neq a \alpha$ nor $b \alpha)$, $\omega^{b} \wedge \varpi_{b \alpha} \wedge \varpi_{a \alpha}$ and $\omega^{a} \wedge \varpi_{a \alpha} \wedge \varpi_{b \alpha}$ are $B_{a}^{\alpha c \beta}, B_{a}^{\alpha a \alpha}-B_{b}^{\alpha b \alpha}$ and $2 B_{a}^{\alpha b \alpha}$ respectively. Hence we get $B_{a}^{\alpha c \beta}=0$ for $c \beta \neq a \alpha$ nor $b \alpha, B_{a}^{\alpha a \alpha}=B_{b}^{\alpha b \alpha}$ and $B_{a}^{\alpha b \alpha}=0$. Moreover, from (6.a.3), we get $B_{i}^{\alpha j \beta}=0$ for $i \neq j, B_{i}^{\alpha i \beta}=0$ for $\alpha \neq \beta$ and $B_{i}^{\alpha i \alpha}=B_{a}^{\alpha a \alpha}\left(=B^{\alpha}\right)$.

In case $s=1$, (6.a.2) reduces to

$$
\omega^{1} \wedge \varpi_{1 \alpha} \wedge\left(B_{1}^{\alpha 1 \beta} \varpi_{1 \beta}+B_{1}^{\alpha i \beta} \varpi_{i \beta}+C_{1}^{\alpha \beta \gamma} \varpi_{\beta \gamma}+\left(E_{1 i}^{\alpha}-\frac{1}{2} D_{i 1}^{\alpha}\right) \omega^{i}\right) \equiv 0 \quad\left(\bmod \quad \mathfrak{M}_{2}^{*}\right) .
$$


Hence we get $B_{1}^{\alpha 1 \beta}=B_{1}^{\beta 1 \alpha}, B_{1}^{\alpha i \beta}=0, C_{1}^{\alpha \beta \gamma}=0$ and $E_{1 i}^{\alpha}=\frac{1}{2} D_{i 1}^{\alpha}$. In case $r=1$, by replacing $\omega^{\alpha}$ by $\omega^{\alpha}+B_{1}^{\alpha 1 \beta} \varpi_{\beta}$, we may assume $B_{1}^{\alpha 1 \beta}=0$. If $r \geqq 2$, from (6.1.3), we get $B_{1}^{\alpha 1 \beta}=0$ for $\alpha \neq \beta, B_{i}^{\alpha j \beta}=0$ for $i \neq j, B_{i}^{\alpha i \beta}=0$ for $\alpha \neq \beta$ and $B_{i}^{\alpha i \alpha}=B_{1}^{\alpha 1 \alpha}\left(=B^{\alpha}\right)$. If we further assume $r \geqq 3$, we have $r-s \geqq 2$. Hence, from (6.1.4), we get $D_{11}^{\alpha}=0$ and $E_{1 i}^{\alpha}=0$, which also implies $D_{i 1}^{\alpha}=0$.

Thus, in case $s \geqq 2$ or $s=1$ and $r \geqq 3$, we see that, by replacing $\omega^{\alpha}$ by $\omega^{\alpha}+B^{\alpha} \varpi_{\alpha},\left(B^{*} .2\right)$ reduces to

$\left(\hat{B}^{*} .2\right) \quad \begin{cases}d \varpi_{a} \equiv & \omega^{\alpha} \wedge \varpi_{a \alpha}+A_{a}^{\alpha \beta} \varpi_{\alpha} \wedge \varpi_{\beta}+B_{a}^{\alpha \lambda} \varpi_{\alpha} \wedge \pi_{\lambda} \\ d \varpi_{i} \equiv \omega^{j} \wedge \pi_{i j}+ & \omega^{\alpha} \wedge \varpi_{i \alpha}+A_{i}^{\alpha \beta} \varpi_{\alpha} \wedge \varpi_{\beta}+B_{i}^{\alpha \lambda} \varpi_{\alpha} \wedge \pi_{\lambda}+B_{i}^{\alpha 1 \beta} \varpi_{\alpha} \wedge \varpi_{1 \beta}\end{cases}$

$\left(\bmod \varpi, \varpi_{1}, \ldots, \varpi_{r}\right)$. Here we may assume $A_{a}^{\alpha \beta}=-A_{a}^{\beta \alpha}$ and $B_{i}^{\alpha 1 \beta}=0$ when $s \geqq 2$. We note that, in case $s=r=1$, we have

$$
d \varpi_{1} \equiv \omega^{\alpha} \wedge \varpi_{1 \alpha}+A_{1}^{\alpha \beta} \varpi_{\alpha} \wedge \varpi_{\beta}+D_{11}^{\alpha} \varpi_{\alpha} \wedge \omega^{1} \quad\left(\bmod \varpi, \varpi_{1}\right) .
$$

Moreover, putting $\mathfrak{M}_{3}^{*}=\left\langle\left\{\varpi, \varpi_{1}, \ldots, \varpi_{n}, \omega^{i}, \omega^{\alpha}(s+1 \leqq i \leqq r, r+1 \leqq \alpha \leqq n)\right\}\right\rangle$, we have

$$
d \varpi \equiv d \varpi_{a} \equiv d \varpi_{i} \equiv 0, \quad d \varpi_{\alpha} \equiv \omega^{b} \wedge \varpi_{b \alpha} \quad\left(\bmod \quad \mathfrak{M}_{3}^{*}\right) .
$$

Then, taking exterior derivatives of both sides of the first equation of $\left(\hat{B}^{*} .2\right)$ and calculating $\bmod \mathfrak{M}_{3}^{*}$, we get

$$
d \omega^{\alpha} \wedge \varpi_{a \alpha}+\omega^{b} \wedge \varpi_{b \alpha} \wedge\left(B_{a}^{\alpha \lambda} \pi_{\lambda}\right) \equiv 0 \quad\left(\bmod \quad \mathfrak{M}_{3}^{*}\right) .
$$

Hence, in case $s \geqq 2$, looking at the coefficient of $\omega^{b} \wedge \varpi_{b \alpha} \wedge \pi_{\lambda}$ for $b \neq a$, we get $B_{a}^{\alpha \lambda}=0$. In case $s=1$, the above equation reduces to

$$
\left(d \omega^{\alpha}-B_{1}^{\alpha \lambda} \omega^{1} \wedge \pi_{\lambda}\right) \wedge \varpi_{1 \alpha} \equiv 0 \quad\left(\bmod \quad \mathfrak{M}_{3}^{*}\right) .
$$

Then, substituting (6.1) into the above equation, we obtain $B_{1}^{\alpha \lambda}=0$. Thus, if $s \geqq 2, s=1$ and $r \geqq 3$ or $s=r=1$ and $C(F)$ is completely integrable, the first equation of $\left(\hat{B}^{*} .2\right)$ reduces to

$$
d \varpi_{a} \equiv \omega^{\alpha} \wedge \varpi_{a \alpha}+A_{a}^{\alpha \beta} \varpi_{\alpha} \wedge \varpi_{\beta} \quad\left(\bmod \varpi, \varpi_{1}, \ldots, \varpi_{r}\right) .
$$

Now we will show that (6.2) further reduces to

$$
d \varpi_{a} \equiv \omega^{\alpha} \wedge \varpi_{a \alpha} \quad\left(\bmod \varpi, \varpi_{1}, \ldots, \varpi_{r}\right),
$$

dividing the proof in the following three cases: (1) $s \geqq 2,(2) s=r=1$ and $C(F)$ is completely integrable, (3) $s=1$ and $r \geqq 3$.

In case (1), putting $\mathfrak{M}_{4}^{*}=\left\langle\left\{\varpi, \varpi_{1}, \ldots, \varpi_{r}, \omega^{\alpha}, \pi_{\lambda}, \varpi_{\alpha} \wedge \varpi_{\beta}\left(1 \leqq \lambda \leqq t_{1}, r+1 \leqq \alpha \leqq \beta \leqq n\right)\right\}\right\rangle$, we have

$$
d \varpi \equiv d \varpi_{a} \equiv d \varpi_{i} \equiv 0, \quad d \varpi_{\alpha} \equiv \omega^{b} \wedge \varpi_{b \alpha}+\varpi_{\beta} \wedge \eta_{\alpha \beta} \quad\left(\bmod \quad \mathfrak{M}_{4}^{*}\right),
$$

for some 1-forms $\eta_{\alpha \beta}$. Then, taking exterior derivatives of both sides of (6.2) and calculating $\bmod \mathfrak{M}_{4}^{*}$, we get

$$
d \omega^{\alpha} \wedge \varpi_{a \alpha}+A_{a}^{\alpha \beta}\left(d \varpi_{\alpha} \wedge \varpi_{\beta}-\varpi_{\alpha} \wedge d \varpi_{\beta}\right) \equiv 0 \quad\left(\bmod \quad \mathfrak{M}_{4}^{*}\right) .
$$

Hence we obtain

$$
d \omega^{\alpha} \wedge \varpi_{a \alpha}+2 A_{a}^{\alpha \beta} \omega^{b} \wedge \varpi_{b \alpha} \wedge \varpi_{\beta} \equiv 0 \quad\left(\bmod \quad \mathfrak{M}_{4}^{*}\right) .
$$

Thus, since $s \geqq 2$, first choose any $a(1 \leqq a \leqq r)$, and find $b$ such that $a \neq b$. Then, looking at the coefficient of $\omega^{b} \wedge \varpi_{b \alpha} \wedge \varpi_{\beta}$, we obtain $A_{a}^{\alpha \beta}=0$.

In case (2), putting $\mathfrak{M}_{5}^{*}=\left\langle\left\{\varpi, \varpi_{1}, \omega^{\alpha}, \varpi_{\alpha} \wedge \varpi_{\beta}(2 \leqq \alpha \leqq \beta \leqq n)\right\}\right\rangle$, we have

$$
d \varpi \equiv d \varpi_{1} \equiv 0, \quad d \varpi_{\alpha} \equiv \omega^{1} \wedge \varpi_{1 \alpha}+\varpi_{\beta} \wedge \eta_{\alpha \beta} \quad\left(\bmod \quad \mathfrak{M}_{5}^{*}\right),
$$


for some 1-forms $\eta_{\alpha \beta}$. Then, taking exterior derivatives of both sides of (6.2) and calculating $\bmod \mathfrak{M}_{5}^{*}$, we get

$$
\left(d \omega^{\alpha}-2 A_{1}^{\alpha \beta} \omega^{1} \wedge \varpi_{\beta}\right) \wedge \varpi_{1 \alpha} \equiv 0 \quad\left(\bmod \quad \mathfrak{M}_{5}^{*}\right)
$$

Thus we obtain

$$
d \omega^{\alpha} \equiv 2 A_{1}^{\alpha \beta} \omega^{1} \wedge \varpi_{\beta} \quad\left(\bmod \varpi, \varpi_{1}, \omega^{\alpha}, \varpi_{1 \alpha}, \varpi_{\alpha} \wedge \varpi_{\beta}(2 \leqq \alpha \leqq \beta \leqq n)\right) .
$$

From $\left(A^{*}\right)$, we have $d \varpi \equiv 0\left(\bmod \varpi, \varpi_{1}, \omega^{2}, \ldots, \omega^{n}\right)$. Hence we have

$$
d \omega^{\alpha} \wedge \varpi_{\alpha} \equiv \omega^{1} \wedge d \varpi_{1} \equiv \omega^{1} \wedge\left(A_{1}^{\alpha \beta} \varpi_{\alpha} \wedge \varpi_{\beta}\right) \quad\left(\bmod \varpi, \varpi_{1}, \omega^{2}, \ldots, \omega^{n}\right) .
$$

Then, substituting (6.3) into the above equation, we obtain $A_{1}^{\alpha \beta} \omega^{1} \wedge \varpi_{\alpha} \wedge \varpi_{\beta} \equiv 0$ (mod $\left.\varpi, \varpi_{1}, \omega^{\alpha}, \varpi_{1 \alpha}(2 \leqq \alpha \leqq n)\right)$, which implies $A_{1}^{\alpha \beta}=0$.

In case (3), putting $\mathfrak{M}_{6}^{*}=\left\langle\left\{\varpi, \varpi_{1}, \ldots, \varpi_{r}, \omega^{\alpha}, \varpi_{1 \alpha}, \pi_{\lambda}, \varpi_{\alpha} \wedge \varpi_{\beta}(r+1 \leqq \alpha \leqq \beta \leqq n, 1 \leqq\right.\right.$ $\left.\left.\left.\lambda \leqq t_{1}\right)\right\}\right\rangle$, we have

$$
d \varpi \equiv d \varpi_{1} \equiv d \varpi_{i} \equiv 0, \quad d \varpi_{\alpha} \equiv \omega^{i} \wedge \varpi_{\alpha i}+\varpi_{\beta} \wedge \eta_{\alpha \beta},
$$

for some 1-forms $\eta_{\alpha \beta}$. Then, taking exterior derivatives of both sides of (6.2) and calculating $\bmod \mathfrak{M}_{6}^{*}$, we get

$$
A_{1}^{\alpha \beta}\left(d \varpi_{\alpha} \wedge \varpi_{\beta}-\varpi_{\alpha} \wedge d \varpi_{\beta}\right) \equiv 2 A_{1}^{\alpha \beta} \omega^{i} \wedge \varpi_{\alpha i} \wedge \varpi_{\beta} \equiv 0 \quad\left(\bmod \quad \mathfrak{M}_{6}^{*}\right) .
$$

This implies $A_{1}^{\alpha \beta}=0$.

Summarizing the discussion above, we obtain

Proposition 6.1. Let $\left(R ; D^{1}, D^{2}\right)$ be a PD manifold of second order, which is regular of type s. Let $F$ be the minimum subspace of $V$ satisfying $\mathfrak{f}^{\perp} \subset S^{2}(F) \subset F \otimes_{S} V$.

Assume that $\{0\} \varsubsetneqq \hat{F} \subset F \varsubsetneqq V$. Let $s$ and $r$ be the dimensions of $\hat{F}$ and $F$ respectively. Then, if $s \geqq 2, s=1$ and $r \geqq 3$ or $s=r=1$ and $C(F)$ is completely integrable, for the covariant system $\tilde{N}^{*}=\left\{\varpi=\varpi_{1}=\cdots=\varpi_{s}=0\right\}$, the following structure equation holds

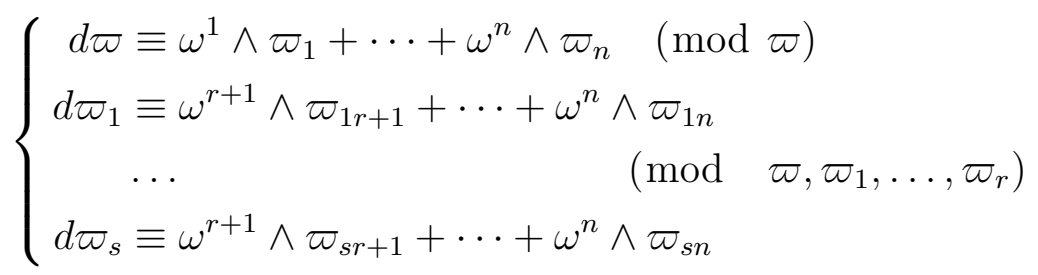

where $\tilde{N}=\left\{\varpi=\varpi_{1}=\cdots=\varpi_{r}=0\right\}$

We note here that, if $s=1$ and $r \leqq 2$, we have $\mathfrak{f}^{\perp}=\left\langle\left\{e_{1} \odot e_{1}\right\}\right\rangle$ or $\left\langle\left\{e_{1} \odot e_{1}, e_{1} \odot e_{2}\right\}\right\rangle$. Furthermore we note that in case $s<r$, by Lemma 5.6, if $C(F)=\operatorname{Ch}(\tilde{N})$, then $C(F) \subset$ $\mathrm{Ch}\left(\tilde{N}^{*}\right)$. Hence, by Proposition 6.1 and Lemma 5.3, we obtain

Proposition 6.2. Let $\left(R ; D^{1}, D^{2}\right)$ be a PD manifold of second order, which is regular of type s. Let $F$ be the minimum subspace of $V$ satisfying $\mathfrak{f}^{\perp} \subset S^{2}(F) \subset F \otimes_{S} V$.

Assume that $\{0\} \varsubsetneqq \hat{F} \subset F \varsubsetneqq V$. Then, except for the cases $\mathfrak{f}^{\perp}=\left\langle\left\{e_{1} \odot e_{1}\right\}\right\rangle$ or $\left\langle\left\{e_{1} \odot\right.\right.$ $\left.\left.e_{1}, e_{1} \odot e_{2}\right\}\right\rangle, C(F)$ is completely integrable and $C(F)=C h(\tilde{N})$. Moreover $C(F) \subset C h\left(\tilde{N}^{*}\right)$ when $s<r$.

Remark 6.1. By Proposition 6.1, for the minimum subspace $F$ of $V$ such that $\hat{F} \neq\{0\}$, except for the cases when $\mathfrak{f}^{\perp}=\left\langle\left\{e_{1} \odot e_{1}\right\}\right\rangle$ or $\left\langle\left\{e_{1} \odot e_{1}, e_{1} \odot e_{2}\right\}\right\rangle$, the assumptions $(i)$ and $(i i)$ for the Two Step Reduction Theorem (Theorem 5.2) are automatically satisfied. For the case when $\mathfrak{f}^{\perp}=\left\langle\left\{e_{1} \odot e_{1}\right\}\right\rangle$, see Theorem 6.1 in the next subsection. 
6.2. Typical Class of Type $\mathfrak{f}^{2}(r)$ and its Generalization. Let $\left(R ; D^{1}, D^{2}\right)$ be a $P D$ manifold of second order satisfying the condition $(C)$, which is regular of type $\mathfrak{f}^{2}(r)$. Namely $\left(R ; D^{1}, D^{2}\right)$ is a $P D$ manifold of second order such that symbol algebra $\mathfrak{s}(v)$ at each point $v \in R$ is isomorphic to $\mathfrak{s}=\mathfrak{s}_{-3} \oplus \mathfrak{s}_{-2} \oplus \mathfrak{s}_{-1}$, where

$$
\mathfrak{s}_{-3}=\mathbb{R}, \quad \mathfrak{s}_{-2}=V^{*} \quad \text { and } \quad \mathfrak{s}_{-1}=V \oplus \mathfrak{f}^{2}(r) .
$$

Here $\mathfrak{f}^{2}(r)$ is given by $\left(\mathfrak{f}^{2}(r)\right)^{\perp}=S^{2}(F) \subset S^{2}(V)$, for a subspace $F$ of $V$ of dimension $r$.

Then, by Proposition 6.1, as the case $s=r \geqq 2$ and the case $s=r=1$ and $C(F)$ is completely integrable, we obtain the structure equations for $\tilde{N}=\left\{\varpi=\varpi_{1}=\cdots=\varpi_{r}=0\right\}$ as follows ;

$$
\left\{\begin{aligned}
d \varpi & \equiv \omega^{\alpha} \wedge \varpi_{\alpha} \\
d \varpi_{a} & \equiv \omega^{\alpha} \wedge \varpi_{a \alpha} \quad\left(\bmod \varpi, \varpi_{1}, \ldots, \varpi_{r}\right) .
\end{aligned}\right.
$$

Hence, by Theorem 5.2, the equivalence of $\left(R ; D^{1}, D^{2}\right)$, which is regular of type $\mathfrak{f}^{2}(r)$, is reducible to that of $\left(Y, D_{N}\right)$ such that $\left(Y, D_{N}\right)$ is a regular differential system of type $\mathfrak{c}^{1}(n-r, r+1)$, where

$$
\mathfrak{c}^{1}(n-r, r+1)=\mathfrak{c}_{-2} \oplus \mathfrak{c}_{-1} \quad \mathfrak{c}_{-2}=W, \quad \mathfrak{c}_{-1}=\hat{V} \oplus W \otimes \hat{V}^{*}
$$

is the symbol algebra of the canonical system on the first order jet space of $n-r$ independent and $r+1$ dependent variables (see $\S 2.5[27]$ ). Here $W$ and $\hat{V}$ are vector spaces of dimension $r+1$ and $n-r$ respectively.

Summarizing the discussion above, by the Second Reduction Theorem (Theorem 5.2), we obtain (Proposition 5.1 and Theorem 5.3 in [25] and $\S 3$ in [23])

Theorem 6.1. Let $\left(R ; D^{1}, D^{2}\right)$ be a PD manifold of second order, which is regular of type $\mathfrak{f}^{2}(r)$.

(1) If $r=1$, assume that $C(F)$ is completely integrable, then the equivalence of $\left(R ; D^{1}, D^{2}\right)$ is reducible to the equivalence of a regular differential system $\left(Y, D_{N}\right)$ of type $\mathfrak{c}^{1}(n-1,2)$.

(2) If $r \geqq 2$, the equivalence of $\left(R ; D^{1}, D^{2}\right)$ is reducible to the equivalence of a regular differential system $\left(Y, D_{N}\right)$ of type $\mathfrak{c}^{1}(n-r, r+1)$, which is locally a space of 1-jets for $n-r$ independent and $r+1$ dependent variables.

Remark 6.2. (1) In case $r=1,\left(R ; D^{1}, D^{2}\right)$, which is of type $\mathfrak{f}^{2}(1)$, is called of (weak) parabolic type and $C(F)$ coincides with the Monge characteristic system. Hence under the assumption that $C(F)$ is completely integrable, $\left(R ; D^{1}, D^{2}\right)$ is called a equation of Goursat type in [25]. Utilizing the above reduction theorem, we discussed the contact equivalences of classes of Goursat type equations $\left(G_{2}\right.$-geometry of second order), which are related to Parabolic Geometries (geometry of $\left(Y, D_{N}\right)$ ) of each exceptional simple Lie Groups (see $\S 6$ in [25]).

(2) In case $r \geqq 2$, since a regular differential system $\left(Y, D_{N}\right)$ of type $\mathfrak{c}^{1}(n-r, r+1)(r+1 \geqq 3)$ is isomorphic to $(J(M, n-r), C)$, where $\operatorname{dim} M=n+1$ (cf. Theorem $1.6[22]),\left(R ; D^{1}, D^{2}\right)$ can

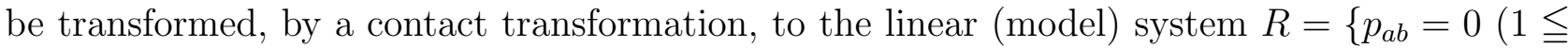
$a, b \leqq r)\}$ (see $\S 3$ in [23] for the detail).

Now, as the generalization of the Typical Class of Type $\mathfrak{f}^{2}(r)$, we will consider a $P D$ manifold $\left(R ; D^{1}, D^{2}\right)$ of second order, which is regular of type $\mathfrak{f}^{2}(r, s)$. Here $\mathfrak{f}^{2}(r, s)$ is given by $\left(\mathfrak{f}^{2}(r, s)\right)^{\perp}=\hat{F} \otimes_{S} F \subset S^{2}(F)$, where $\hat{F} \subset F$ are subspaces of $V$ of dimension $s$ and $r$ respectively. Namely let us fix a point $v \in R$. Then, as in $\S 6.1$, there exists a coframe $\left\{\varpi, \varpi_{1}, \ldots, \varpi_{n}, \omega^{1}, \ldots, \omega^{n}, \varpi_{a \beta}(1 \leqq a \leqq s, r+1 \leqq \beta \leqq n), \varpi_{k l}(s+1 \leqq k \leqq l \leqq n)\right\}$ defined around $v \in R$ such that

$$
\begin{aligned}
& D^{1}=\{\varpi=0\}, \quad D^{2}=\left\{\varpi=\varpi_{1}=\cdots=\varpi_{n}=0\right\}, \quad \tilde{N}=\tilde{N}(F)=\left\{\varpi=\varpi_{1}=\cdots=\varpi_{r}=0\right\}, \\
& \tilde{N}^{*}=\tilde{N}(\hat{F})=\left\{\varpi=\varpi_{1}=\cdots=\varpi_{s}=0\right\}, \tilde{N}^{\perp}=\left\{\varpi=\varpi_{1}=\cdots=\varpi_{n}=\omega^{r+1}=\cdots=\omega^{n}=0\right\},
\end{aligned}
$$


and the following structure equations hold;

$$
d \varpi \equiv \omega^{1} \wedge \varpi_{1}+\cdots+\omega^{n} \wedge \varpi_{n} \quad(\bmod \varpi),
$$

$$
\left\{\begin{array}{cc}
d \varpi_{a} \equiv & \sum_{\alpha=r+1}^{n} \omega^{\alpha} \wedge \varpi_{a \alpha}(1 \leqq a \leqq s), \\
d \varpi_{i} \equiv & \sum_{j=s+1}^{r} \omega^{j} \wedge \varpi_{i j}+\sum_{\alpha=r+1}^{n} \omega^{\alpha} \wedge \varpi_{i \alpha}(s+1 \leqq i \leqq r), \\
d \varpi_{\alpha} \equiv \sum_{a=1}^{s} \omega^{a} \wedge \varpi_{a \alpha}+\sum_{i=s+1}^{r} \omega^{i} \wedge \varpi_{i \alpha}+\sum_{\beta=r+1}^{n} \omega^{\beta} \wedge \varpi_{\alpha \beta}(r+1 \leqq \alpha \leqq n),
\end{array}\right.
$$

$\left(\bmod \varpi, \varpi_{1}, \ldots, \varpi_{n}\right)$, where $\varpi_{k l}=\varpi_{l k}$. Furthermore, we have

$$
\begin{array}{r}
C(F)=\left\{\varpi=\varpi_{1}=\cdots=\varpi_{n}=\omega^{s+1}=\cdots=\omega^{n}=\varpi_{i j}=\varpi_{k \alpha}=0\right. \\
(s+1 \leqq i \leqq j \leqq r, 1 \leqq k \leqq r, r+1 \leqq \alpha \leqq n)\},
\end{array}
$$

By the calculation in $\S 6.1$, in case $s \geqq 2$, we have

$$
\begin{cases}d \varpi_{a} & \equiv \\ d \varpi_{i} & \equiv \omega^{j} \wedge \varpi_{i j}+\omega^{\alpha} \wedge \varpi_{a \alpha} \\ & \varpi_{i \alpha}+A_{i}^{\alpha \beta} \varpi_{\alpha} \wedge \varpi_{\beta}+B_{i}^{\alpha j k} \varpi_{\alpha} \wedge \varpi_{j k}\end{cases}
$$

$\left(\bmod \varpi, \varpi_{1}, \ldots, \varpi_{r}\right)$, where we may assume $A_{i}^{\alpha \beta}=-A_{i}^{\beta \alpha}$. Putting $\tilde{\mathfrak{M}}_{1}=\left\langle\left\{\varpi, \varpi_{1}, \ldots, \varpi_{n}, \omega^{i}\right.\right.$, $\left.\left.\omega^{\alpha}(s+1 \leqq i \leqq r, r+1 \leqq \alpha \leqq \beta \leqq n)\right\}\right\rangle$ and by (6.1), we have

$$
d \varpi \equiv d \varpi_{a} \equiv d \varpi_{i} \equiv d \omega^{\alpha} \equiv 0, \quad d \varpi_{\alpha} \equiv \omega^{b} \wedge \varpi_{b \alpha} \quad\left(\bmod \quad \tilde{\mathfrak{M}}_{1}\right) .
$$

Then, taking exterior derivatives of both sides of the second equation of $(\tilde{B} .2)$ and calculating $\bmod \tilde{\mathfrak{M}}_{1}$, we get

$$
d \omega^{j} \wedge \varpi_{i j}+\omega^{b} \wedge \varpi_{b \alpha} \wedge\left(B_{i}^{\alpha j k} \varpi_{j k}\right) \equiv 0 \quad\left(\bmod \quad \tilde{\mathfrak{M}}_{1}\right) .
$$

Hence we have $B_{i}^{\alpha j k}=0$ if $i \notin\{j, k\}$ and $\left(d \omega^{j}+B_{i}^{\alpha i j} \omega^{b} \wedge \varpi_{b \alpha}\right) \wedge \varpi_{i j} \equiv 0\left(\bmod \quad \tilde{\mathfrak{M}}_{1}\right)$. Thus we obtain

$$
d \omega^{j} \equiv-B_{i}^{\alpha i j} \omega^{b} \wedge \varpi_{b \alpha} \quad\left(\bmod \quad \tilde{\mathfrak{M}}_{1}\right) \quad \text { for } \quad i=s+1, \ldots, r .
$$

Then, putting $B^{\alpha j}=B_{i}^{\alpha i j}(i=s+1, \ldots, r)$, and replacing $\omega^{j}$ and $\omega^{\alpha}$ by $\omega^{j}+B^{\alpha j} \varpi_{\alpha}$ and $\omega^{\alpha}+B^{\alpha j} \varpi_{j}$ respectively, we may assume $B_{i}^{\alpha j k}=0$.

Moreover, putting $\tilde{\mathfrak{M}}_{2}=\left\langle\left\{\varpi, \varpi_{1}, \ldots, \varpi_{r}, \omega^{i}, \omega^{\alpha}, \varpi_{i j}, \varpi_{i \alpha}, \varpi_{\alpha} \wedge \varpi_{\beta}(s+1 \leqq i \leqq j \leqq r, r+1 \leqq\right.\right.$ $\alpha \leqq \beta \leqq n)\}\rangle$, we have

$$
d \varpi \equiv d \varpi_{a} \equiv d \varpi_{i} \equiv 0, \quad d \varpi_{\alpha} \equiv \omega^{b} \wedge \varpi_{b \alpha}+\varpi_{\beta} \wedge \eta_{\alpha \beta} \quad\left(\bmod \quad \tilde{\mathfrak{M}}_{2}\right),
$$

for some 1-forms $\eta_{\alpha \beta}$. Then, taking exterior derivatives of both sides of the second equation of $(\tilde{B} .2)$ and calculating mod $\tilde{\mathfrak{M}}_{2}$, we get

$$
A_{i}^{\alpha \beta}\left(d \varpi_{\alpha} \wedge \varpi_{\beta}-\varpi_{\alpha} \wedge d \varpi_{\beta}\right) \equiv 2 A_{i}^{\alpha \beta} \omega^{b} \wedge \varpi_{b \alpha} \wedge \varpi_{\beta} \equiv 0 \quad\left(\bmod \quad \tilde{\mathfrak{M}}_{2}\right),
$$

which implies $A_{i}^{\alpha \beta}=0$.

Thus, by the Second Reduction Theorem (Theorem 5.2), we obtain 
Theorem 6.2. Let $\left(R ; D^{1}, D^{2}\right)$ be a PD manifold of second order, which is regular of type $\mathfrak{f}^{2}(r, s)$.

Then, if $2 \leqq s<r$, the equivalence of $\left(R ; D^{1}, D^{2}\right)$ is reducible, by Theorem 5.2, to the equivalence of a regular differential system $\left(Y, D_{N}\right)$, where $D_{N}=\left\{\varpi=\varpi_{1}=\cdots=\varpi_{r}=0\right\}$ such that the following structure equation holds;

$$
\left\{\begin{array}{cc}
d \varpi & \sum_{\alpha=r+1}^{n} \omega^{\alpha} \wedge \varpi_{\alpha}, \\
d \varpi_{a} \equiv & \sum_{\alpha=r+1}^{n} \omega^{\alpha} \wedge \varpi_{a \alpha}(1 \leqq a \leqq s), \quad\left(\bmod \varpi, \varpi_{1}, \ldots, \varpi_{r}\right) \\
d \varpi_{i} \equiv \sum_{j=s+1}^{r} \omega^{j} \wedge \varpi_{i j}+\sum_{\alpha=r+1}^{n} \omega^{\alpha} \wedge \varpi_{i \alpha}(s+1 \leqq i \leqq r),
\end{array}\right.
$$

6.3. Typical Class of Type $\mathfrak{f}^{1}(r)$ and its Generalization. In this subsection, as the generalization of the Typical Class of Type $\mathfrak{f}^{1}(r)$, we will consider a $P D$ manifold $\left(R ; D^{1}, D^{2}\right)$ of second order, which is regular of type $\mathfrak{s}$ such that $\mathfrak{s}=\mathfrak{s}_{-3} \oplus \mathfrak{s}_{-2} \oplus \mathfrak{s}_{-1}$ satisfies the following condition: For $\mathfrak{s}_{-3}=\mathbb{R}, \mathfrak{s}_{-2}=V^{*}$ and $\mathfrak{s}_{-1}=V \oplus \mathfrak{f}, \mathfrak{f} \subset S^{2}\left(V^{*}\right)$,

(F.1) There exist subspaces $E$ and $H$ of $V$ of dimension $r$ and $n-r$ respectively such that

$$
V=E \oplus H, \quad E \otimes H \subset \mathfrak{f}^{\perp} \subset E \otimes_{S} V .
$$

Here $\mathfrak{f}^{1}(r)$ is given by $\left(\mathfrak{f}^{1}(r)\right)^{\perp}=E \otimes H$. Namely let us fix a point $v \in R$. Then, as in $\S 6.1$, there exists a coframe $\left\{\varpi, \varpi_{1}, \ldots, \varpi_{n}, \omega^{1}, \ldots, \omega^{n}, \pi_{\lambda}, \varpi_{\alpha \beta}(1 \leqq \lambda \leqq t, r+1 \leqq \alpha \leqq \beta \leqq n)\right\}$ $\left(t=\operatorname{dim} \mathfrak{f}-\operatorname{dim} S^{2}\left(E^{\perp}\right)\right)$ and 1-forms $\left\{\pi_{i j}(1 \leqq i, j \leqq r)\right\}$ defined around $v \in R$ such that

$$
D^{1}=\{\varpi=0\}, \quad D^{2}=\left\{\varpi=\varpi_{1}=\cdots=\varpi_{n}=0\right\},
$$

$\tilde{N}=\tilde{N}(E)=\left\{\varpi=\varpi_{1}=\cdots=\varpi_{r}=0\right\}, \tilde{N}^{\perp}=\left\{\varpi=\varpi_{1}=\cdots=\varpi_{n}=\omega^{r+1}=\cdots=\omega^{n}=0\right\}$, and the following structure equations hold;

$$
\begin{aligned}
& d \varpi \equiv \omega^{1} \wedge \varpi_{1}+\cdots+\omega^{n} \wedge \varpi_{n} \quad(\bmod \varpi),
\end{aligned}
$$

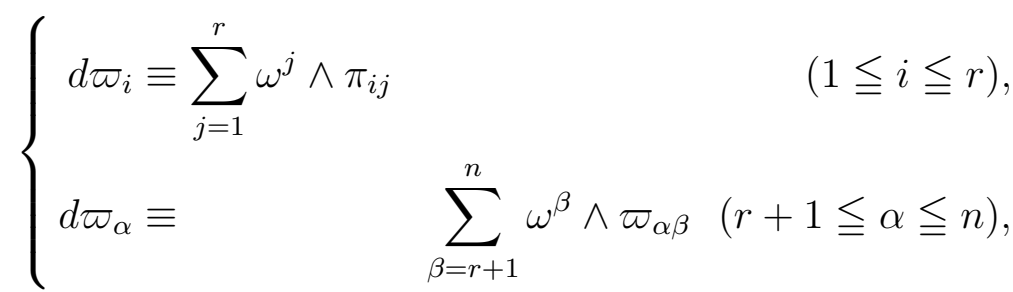

$\left(\bmod \varpi, \varpi_{1}, \ldots, \varpi_{n}\right)$, where $\varpi_{\alpha \beta}=\varpi_{\beta \alpha}, \pi_{i j}=\pi_{j i}$, and $\left\{\pi_{\lambda}(1 \leqq \lambda \leqq t)\right\}$ is a basis of $\left\langle\left\{\pi_{i j}(1 \leqq\right.\right.$ $i, j \leqq r)\}\rangle\left(\bmod \varpi, \varpi_{1}, \ldots, \varpi_{n}\right)$. Furthermore, we have

$$
\mathfrak{f}(E)=\left\{\varpi=\varpi_{1}=\cdots=\varpi_{n}=\omega^{1}=\cdots=\omega^{n}=\pi_{i j}=0(1 \leqq i \leqq j \leqq r)\right\} .
$$

From $(\hat{B} .1)$, we have

$$
\begin{aligned}
d \varpi_{i} \equiv \omega^{j} \wedge \pi_{i j} & +A_{i}^{\alpha \beta} \varpi_{\alpha} \wedge \varpi_{\beta}+B_{i}^{\alpha \lambda} \varpi_{\alpha} \wedge \pi_{\lambda}+C_{i}^{\alpha \beta \gamma} \varpi_{\alpha} \wedge \varpi_{\beta \gamma} \\
& +D_{i j}^{\alpha} \varpi_{\alpha} \wedge \omega^{j}+E_{i \beta}^{\alpha} \varpi_{\alpha} \wedge \omega^{\beta} \quad\left(\bmod \varpi, \varpi_{1}, \ldots, \omega_{r}\right) .
\end{aligned}
$$

First, replacing $\varpi_{i}$ by $\varpi-E_{i r+1}^{r+1} \varpi$, we may assume $E_{i r+1}^{r+1}=0$. Putting $\hat{\mathfrak{M}}_{1}=\left\langle\left\{\varpi, \varpi_{1}, \ldots, \varpi_{n}, \omega^{i}\right.\right.$, $\left.\left.\pi_{\lambda}(1 \leqq i \leqq r, 1 \leqq \lambda \leqq t)\right\}\right\rangle$, we have

$$
d \varpi \equiv d \varpi_{i} \equiv 0, \quad d \varpi_{\alpha} \equiv \omega^{\beta} \wedge \varpi_{\alpha \beta} \quad\left(\bmod \quad \hat{\mathfrak{M}}_{1}\right) .
$$


Then, taking exterior derivatives of both sides of $(\hat{B} .2)$ and calculating $\bmod \hat{\mathfrak{M}}_{1}$, we get

$$
\omega^{\beta} \wedge \varpi_{\alpha \beta} \wedge\left(C_{i}^{\alpha \beta \gamma} \varpi_{\beta \gamma}+E_{i \beta}^{\alpha} \omega^{\beta}\right) \equiv 0 \quad\left(\bmod \quad \hat{\mathfrak{M}}_{1}\right) .
$$

Under the assumption $r \leqq n-2$, this implies $C_{i}^{\alpha \beta \gamma}=E_{i \beta}^{\alpha}=0$ (see the proof of Lemma 2.1 in [23] for the detail ). Hence, by Lemma 5.3. (1), we obtain $\mathfrak{f}(E)=\operatorname{Ch}\left(D^{1}\right) \cap \operatorname{Ch}(\tilde{N})$. Let $\left(W ; C^{*}, N\right)$ be the $I G$ manifold of corank $r$ associated with $\left(R ; D^{1}, D^{2}, \tilde{N}(E)\right)$ and $\left(R_{W} ; D_{W}^{1}, D_{W}^{2}\right)$ be the Lagrange Grassmann bundle over $\left(W ; C^{*}, N\right)$. Then, by Theorem 5.1. (1), $\kappa_{1}:\left(R ; D^{1}, D^{2}\right) \rightarrow$ $\left(R(W) ; D_{W}^{1}, D_{W}^{2}\right)$ is a local isomorphism. Thus the local equivalence of $\left(R ; D^{1}, D^{2}\right)$ is reducible to the local equivalence of $\left(W ; C^{*}, N\right)$. By the condition $(F .1)$ of the symbol algebra, we have

$$
S^{2}\left(E^{\perp}\right) \subset \mathfrak{f} \subset S^{2}\left(H^{\perp}\right) \oplus S^{2}\left(E^{\perp}\right) .
$$

Hence we get $\mathfrak{f} \cap\left(E^{\perp} \otimes_{S} V^{*}\right)=S^{2}\left(E^{\perp}\right)=\mathfrak{f}(E)=$ Ker $\eta_{*}$, which implies, by Lemma 3.1 (4), $S(N)=\{0\}$ on $W$.

Summarizing the discussion above, we obtain

Proposition 6.3. Let $\left(R ; D^{1}, D^{2}\right)$ be a PD manifold of second order, which is regular of type $\mathfrak{s}$ such that the symbol subspace $\mathfrak{f} \subset S^{2}\left(V^{*}\right)$ satisfies the condition $(F .1)$. Let $\left(W ; C^{*}, N\right)$ be the $I G$ manifold of corank $r$ associated with $\left(R ; D^{1}, D^{2}, \tilde{N}(E)\right)$ and $\left(R_{W} ; D_{W}^{1}, D_{W}^{2}\right)$ be the Lagrange Grassmann bundle over $\left(W ; C^{*}, N\right)$. Then $\kappa_{1}:\left(R ; D^{1}, D^{2}\right) \rightarrow\left(R(W) ; D_{W}^{1}, D_{W}^{2}\right)$ is a local isomorphism and $S(N)=\{0\}$ on $W$.

Conversely we will now consider an $I G$ manifold $\left(W ; C^{*}, N\right)$ of corank $r$ satisfying $S(N)=$ $\{0\}$. We assume that $W$ is regular with respect to $\mathrm{Ch}\left(C^{*}\right)$ as in $\S 4.1$. Let $\left(R(W) ; D_{W}^{1}, D_{W}^{2}\right)$ be the Lagrange Grassmann bundle over $\left(W ; C^{*}, N\right)$. Then, by Lemma 4.1 and $4.2,\left(R(W) ; D_{W}^{1}, D_{W}^{2}\right)$ is, globally, a $P D$ manifold of second order and $\zeta: R(W) \rightarrow L(J)$ is an immersion.

Moreover, by Lemma 3.1 (4) (see also the last paragraph of $\S 4.2$ ), the condition $S(N)=\{0\}$ implies that

$$
S\left(N_{W}\right)(\hat{v})=\mathfrak{f}(\hat{v}) \cap\left(E(\hat{v})^{\perp} \otimes_{S} V(\hat{v})^{*}\right)=\operatorname{Ker} \tau_{*}=S^{2}\left(E(\hat{v})^{\perp}\right),
$$

at each $\hat{v} \in R(W)$, where $E(\hat{v})=V(\hat{v}) \cap N_{W}^{\perp}(\hat{v})$. Equivalently we have

$$
\mathfrak{f}(\hat{v})^{\perp}+S^{2}(E(\hat{v}))=E(\hat{v}) \otimes_{S} V(\hat{v}) \quad \text { at each } \quad \hat{v} \in R(W) .
$$

For a complimentary subspace $H(\hat{v}), V(\hat{v})=E(\hat{v}) \oplus H(\hat{v})$, we have $E(\hat{v}) \otimes_{S} V(\hat{v})=S^{2}(E(\hat{v}) \oplus$ $E(\hat{v}) \otimes H(\hat{v})$. Thus the condition $(F .1)$ in this case is the existence of a complimentary subspace $H(\hat{v})$ such that $\mathfrak{f}(\hat{v})^{\perp} \supset E(\hat{v}) \otimes H(\hat{v})$.

\section{Construction of $\left(W(Y) ; C_{Y}^{*}, N_{Y}\right)$ And $\left(R(Y) ; D_{Y}^{1}, D_{Y}^{2}\right)$}

7.1. Case $N^{*}=N$. Starting from a regular differential system $\left(Y, D_{N}\right)$, we will construct an $I G$ manifold $\left(W(Y) ; C_{Y}^{*}, N_{Y}\right)$ and the Lagrange Grassmann bundle $R(Y)=R(W(Y)$ ) over $\left(W(Y) ; C_{Y}^{*}, N_{Y}\right)$ and will examine the condition when $\left(R(Y) ; D_{Y}^{1}, D_{Y}^{2}\right)$ becomes a $P D$ manifold of second order, where $D_{Y}^{1}$ and $D_{Y}^{2}$ are canonical systems on $R(Y)$.

Let $\left(Y, D_{N}\right)$ be a differential system satisfying the following condition;

(Y.1) $\quad D_{N}$ is a differential system of codimension $r+1$ such that $\mathrm{Ch}\left(D_{N}\right)$ is trivial.

We assume that $D_{N}$ is of constant Engel half-rank (see [4] II $\S 4$ ) and let $t$ be the Engel half-rank of $D_{N}$. Let $\left\{\varpi_{0}, \ldots, \varpi_{r}\right\}$ be a local defining 1-forms of $\left(Y, D_{N}\right)$ on a neighborhood $U$ of $y_{o} \in Y$. Then, for a section $\varpi \in \Gamma\left(D_{N}^{\perp}\right)$, we have

$$
(d \varpi)^{t+1} \equiv 0 \quad\left(\bmod \varpi_{0}, \ldots, \varpi_{r}\right),
$$

on $U$ and $(d \hat{\omega})^{t} \not \equiv 0$ for some $\hat{\varpi} \in \Gamma\left(D_{N}^{\perp}\right)$. Here we may assume $\left(d \varpi_{0}\right)^{t} \not \equiv 0$ around $y_{o} \in Y$. 
Now let us consider the collection $\hat{W}(Y)$ of hyperplanes $w$ in each tangent space $T_{y}(Y)$ at $y \in Y$ which contains the fibre $D_{N}(y)$ of $D_{N}$;

$$
\begin{gathered}
\hat{W}(Y)=\bigcup_{y \in Y} \hat{W}_{y} \subset J(Y, m-1), \\
\hat{W}_{y}=\left\{w \in \operatorname{Gr}\left(T_{y}(Y), m-1\right) \mid w \supset D_{N}(y)\right\} \cong \mathbb{P}\left(T_{y}(Y) / D_{N}(y)\right)=\mathbb{P}^{r},
\end{gathered}
$$

where $m=\operatorname{dim} Y$. Moreover $C_{Y}^{*}$ is the canonical system obtained by the Grassmannian construction and $N_{Y}$ is the lift of $D_{N}$. Precisely, $C_{Y}^{*}$ and $N_{Y}$ are given by

$$
C_{Y}^{*}(w)=\nu_{*}^{-1}(w) \supset N_{Y}(w)=\nu_{*}^{-1}\left(D_{N}(y)\right),
$$

for each $w \in \hat{W}(Y)$ and $y=\nu(w)$, where $\nu: \hat{W}(Y) \rightarrow Y$ is the projection.

We will now examine the condition for $\left(\hat{W}(Y) ; C_{Y}^{*}, N_{Y}\right)$ to be an $I G$ manifold of corank $r$. Let us consider

$$
\varpi=\varpi_{0}+\lambda_{1} \varpi_{1}+\cdots+\lambda_{r} \varpi_{r}
$$

on $U$. Namely we consider a point $w \in \hat{W}(Y)$ such that $w=\{\varpi=0\} \subset T_{y}(Y)$, where $y=\nu(w) \in U$. Here $\left(\lambda_{1}, \ldots, \lambda_{r}\right)$ constitutes an inhomogeneous coordinate of the fibres of $\nu: \hat{W}(Y) \rightarrow Y$. Denoting the pullback on $\hat{W}(Y)$ of 1-forms on $Y$ by the same symbol, we have

$$
C_{Y}^{*}=\{\varpi=0\},
$$

and

$$
d \varpi=d \varpi_{0}+\sum_{i=1}^{r} \lambda_{i} d \varpi_{i}+\sum_{i=1}^{r} d \lambda_{i} \wedge \varpi_{i} .
$$

on $\nu^{-1}(U)$. By the Engel half-rank condition, we have, around $y_{0}$,

$$
\begin{aligned}
d \varpi_{0}+\sum_{i=1}^{r} \lambda_{i} d \varpi_{i} & \equiv \sum_{\alpha=1}^{t} \tilde{\omega}^{\alpha} \wedge \tilde{\varpi}^{\alpha} \quad\left(\bmod \quad \varpi_{0}, \ldots, \varpi_{r}\right) \\
& \equiv \sum_{\alpha=1}^{t} \tilde{\omega}^{\alpha} \wedge \tilde{\varpi}^{\alpha}+\sum_{i=1}^{r} \gamma_{i} \wedge \varpi_{i} \quad(\bmod \varpi)
\end{aligned}
$$

where $\tilde{\omega}^{\alpha}, \tilde{\omega}^{\alpha}(1 \leqq \alpha \leqq t), \gamma_{i}(1 \leqq i \leqq r)$ are 1 -forms on $\mathrm{U}$ defined around $y_{0}$ such that $\left\{\varpi_{0}, \ldots, \varpi_{r}, \tilde{\omega}^{\alpha}, \tilde{\varpi}^{\alpha}(1 \leqq \alpha \leqq t)\right\}$ are linearly independent at each point. Then we have

$$
d \varpi \equiv \sum_{\alpha=1}^{t} \tilde{\omega}^{\alpha} \wedge \tilde{\varpi}^{\alpha}+\sum_{i=1}^{r}\left(d \lambda_{i}+\gamma_{i}\right) \wedge \varpi_{i} \quad(\bmod \varpi) .
$$

around $w_{0}=\left\{\varpi_{0}=0\right\} \in \nu^{-1}(U)$. Hence we have

$$
\operatorname{Ch}\left(C_{Y}^{*}\right)(w)=\left\{\varpi=\varpi_{i}=d \lambda_{i}+\gamma_{i}=\tilde{\omega}^{\alpha}=\tilde{\varpi}^{\alpha}=0 \quad(1 \leqq i \leqq r, 1 \leqq \alpha \leqq t)\right\} .
$$

around $w_{0} \in \nu^{-1}(U)$. Thus the following subset $W(Y)$ of $\hat{W}(Y)$ is an open (dense) subset of $\hat{W}(Y)$;

$$
W(Y)=\left\{w \in \hat{W}(Y) \mid \operatorname{corank} \operatorname{Ch}\left(C_{Y}^{*}\right)(w)=2 n+1\right\}
$$

where $n=r+t$.

Now we claim

Proposition 7.1. Let $\left(Y, D_{N}\right)$ be a differential system satisfying $(Y .1)$ and let $t$ be the Engel half-rank of $D_{N}$. Then $\left(W(Y) ; C_{Y}^{*}, N_{Y}\right)$ is an IG manifold of corank $r$, where $n=r+t$. Moreover

(1) $N_{Y}^{\perp}=C h\left(N_{Y}\right) \oplus C h\left(C_{Y}^{*}\right) \quad$ and $\quad S\left(N_{Y}\right)=C h\left(C_{Y}^{*}\right)$.

(2) $\partial N_{Y}^{\perp} \subset N_{Y}$, hence $N_{Y}^{*}=N_{Y}$, where $N_{Y}^{*}=\partial N_{Y}^{\perp}+N_{Y}$. 
Proof. Notations being as above, we have on a neighborhood of $w_{0} \in \nu^{-1}(U)$

$$
C_{Y}^{*}=\{\varpi=0\}, \quad N_{Y}=\left\{\varpi=\varpi_{1}=\cdots=\varpi_{r}=0\right\},
$$

and

$$
d \varpi \equiv \sum_{\alpha=1}^{t} \tilde{\omega}^{\alpha} \wedge \tilde{\varpi}^{\alpha}+\sum_{i=1}^{r}\left(d \lambda_{i}+\gamma_{i}\right) \wedge \varpi_{i} \quad(\bmod \quad \varpi)
$$

Hence we get

$$
\operatorname{Ch}\left(C_{Y}^{*}\right)=\left\{\varpi=\varpi_{i}=\tilde{\varpi}^{\alpha}=\tilde{\omega}^{\alpha}=d \lambda_{i}+\gamma_{i}=0 \quad(1 \leqq i \leqq r, 1 \leqq \alpha \leqq t)\right\},
$$

and

$$
N_{Y}^{\perp}=\left\{\varpi=\varpi_{i}=\tilde{\varpi}^{\alpha}=\tilde{\omega}^{\alpha}=0 \quad(1 \leqq i \leqq r, 1 \leqq \alpha \leqq t)\right\} .
$$

Moreover, from $\operatorname{Ch}\left(D_{N}\right)=\{0\}$, we have $\operatorname{Ch}\left(N_{Y}\right)=\operatorname{Ker} \nu_{*}$, which implies $\operatorname{Ch}\left(C_{Y}^{*}\right) \cap \operatorname{Ch}\left(N_{Y}\right)=$ $\{0\}$. Thus $\left(W(Y) ; C_{Y}^{*}, N_{Y}\right)$ is an $I G$ manifold of corank $r$.

(1) From $\mathrm{Ch}\left(N_{Y}\right) \subset N_{Y}^{\perp}$ and the rank count, we have

$$
N_{Y}^{\perp}=\mathrm{Ch}\left(N_{Y}\right) \oplus \mathrm{Ch}\left(C_{Y}^{*}\right) .
$$

Then it follows that

$$
S\left(N_{Y}\right)(w)=\left\{X \in \operatorname{Ch}\left(C_{Y}^{*}\right)(w) \mid\left[X, \Gamma\left(N_{Y}^{\perp}\right)\right] \subset \Gamma\left(N_{Y}\right)\right\} \supset \operatorname{Ch}\left(C_{Y}^{*}\right)(w) .
$$

(2) $\partial N_{Y}^{\perp} \subset N_{Y}$ follows immediately from $N_{Y}^{\perp}=\mathrm{Ch}\left(N_{Y}\right) \oplus \operatorname{Ch}\left(C_{Y}^{*}\right)$. This completes the proof of Proposition.

Here we observe that, when $\left(Y, D_{N}\right)$ is obtained from a $P D$ manifold of second order such that $E=\hat{E}$ as in Theorem 5.2, the Engel-half rank of $\left(Y, D_{N}\right)$ equals to $t=n-r$. This can be checked as follows; Let $\left(W ; C^{*}, N\right)$ be the associated $I G$ manifold of corank $r$ such that $Y=W / \mathrm{Ch}(N)$ is the leaf space. Then we have a map $\kappa_{2}: W \rightarrow \hat{W}(Y)$ as in $\S 5.3$, which is an immersion. Hence we see $\kappa_{2}(W) \subset W(Y)$ and $\kappa_{2}:\left(W ; C^{*}, N\right) \rightarrow\left(W(Y) ; C_{Y}^{*}, N_{Y}\right)$ is a local isomorphism of $I G$ manifolds.

Now we consider the Lagrange Grassmann bundle $R(Y)=R(W(Y))$ over $\left(W(Y) ; C_{Y}^{*}, N_{Y}\right)$ :

$$
R(Y)=\bigcup_{w \in W(Y)} R_{w}, \quad R_{w}=\left\{\hat{v} \subset N_{Y}(w)\left|\gamma_{w}\right|_{\hat{v}}=0, \quad \hat{v} \text { is maximal }\right\} .
$$

Let $D_{Y}^{2}$ be the canonical system on $R(Y)$ and let $D_{Y}^{1}$ and $\hat{N}_{Y}$ be the lifts of $C_{Y}^{*}$ and $N_{Y}$ respectively, i.e.,

$$
D_{Y}^{1}(\hat{v})=\tau_{*}^{-1}\left(C_{Y}^{*}(w)\right), \quad D_{Y}^{2}(\hat{v})=\tau_{*}^{-1}(\hat{v}), \quad \hat{N}_{Y}(\hat{v})=\tau_{*}^{-1}\left(N_{Y}(w)\right),
$$

where $\tau: R(Y) \rightarrow W(Y)$ is the projection and $w=\tau(\hat{v})$.

In general, in order to see whether $\left(R(Y) ; D_{Y}^{1}, D_{Y}^{2}\right)$ is a $P D$ manifold of second order, we must check the condition $A(\hat{v})=\{0\}$ for each $\hat{v} \in R(Y)$, utilizing, e.g., Lemma 4.2 together with the structure equation of $D_{N}$. Here we will discuss two extreme cases in the following.

First we impose the following condition for $\left(Y, D_{N}\right)$;

$(Y .2) \quad \operatorname{rank} D_{N}=2 t$, where $t$ is the Engel half-rank of $D_{N}$.

This condition is equivalent to the condition that $\left\{\varpi_{0}, \ldots, \varpi_{r}, \tilde{\varpi}^{1}, \ldots, \tilde{\varpi}^{t}, \tilde{\omega}^{1}, \ldots, \tilde{\omega}^{t}\right\}$ forms a coframe on a neighborhood around $y_{o} \in Y$ in the above notation. Then we have 
Proposition 7.2. Let $\left(Y, D_{N}\right)$ be a differential system satisfying $(Y .1)$, and let $t$ be the Engel half-rank of $D_{N}$. Then

(1) $\left(W(Y), C_{Y}^{*}\right)$ is a contact manifold of dimension $2 n+1$ if and only if $(Y .2)$ holds.

(2) If $(Y .2)$ holds, $\left(W(Y) ; C_{Y}^{*}, N_{Y}\right)$ is an IG manifold of corank $r$, where $n=r+t$, such that $N_{Y}^{\perp}=C h\left(N_{Y}\right)$ and that $\left(R(Y) ; D_{Y}^{1}, D_{Y}^{2}\right)$ is, globally, a PD manifold of second order satisfying $\mathfrak{f}(\hat{v}) \cong S^{2}\left(E^{\perp}\right)$ for some $r$-dimensional subspace $E$ of $V$ at each $\hat{v} \in R(Y)$.

Proof. (1) $\left(W(Y), C_{Y}^{*}\right)$ is a contact manifold of dimension $2 n+1$ if and only if $\mathrm{Ch}\left(C_{Y}^{*}\right)=\{0\}$. By (7.1), this is equivalent to the condition that $\left\{\varpi, \varpi_{i}, d \lambda_{i}+\gamma_{i}, \tilde{\omega}^{\alpha}, \tilde{\varpi}^{\alpha}(1 \leqq i \leqq r, 1 \leqq\right.$ $\alpha \leqq t)\}$ is a coframe around $w_{o} \in \nu^{-1}(U)$, which is also equivalent to the condition that $\left\{\varpi_{0}, \ldots, \varpi_{r}, \tilde{\varpi}^{1}, \ldots, \tilde{\varpi}^{t}, \tilde{\omega}^{1}, \ldots, \tilde{\omega}^{t}\right\}$ is a coframe on a neighborhood of $y_{o} \in Y$.

(2) If (Y.2) holds, $N_{Y}^{\perp}=\mathrm{Ch}\left(N_{Y}\right)$ follows from Proposition 7.1 and the last assertion follows from $S\left(N_{Y}\right)=\operatorname{Ch}\left(C_{Y}^{*}\right)=\{0\}$ and Lemma 4.2.

Unfortunately, in view of Case (3) of Theorem in [23] (see also $\S 6.1[27]$ ), these $R(Y)$ are inevitably incompatible systems, i.e., $R(Y)$ does not satisfy the compatibility condition $(C)$ in $\S 3.1$ ( or $\S 4.2$ ). We will give some examples of this case in $\S 8.3$.

Secondly we assume the following condition for $\left(Y, D_{N}\right)$ (see [4] II $\S 4$ );

(Y.3) The Cartan rank of $D_{N}$ coincides with the Engel half-rank of $D_{N}$.

Under this condition, we have a local defining 1-forms $\left\{\varpi_{0}, \ldots, \varpi_{r}\right\}$ of $\left(Y, D_{N}\right)$ on a neighborhood $U$ of $y_{0} \in Y$ such that the structure equation of the following form holds:

$$
d \varpi_{i} \equiv \omega^{1} \wedge \pi_{i 1}+\cdots+\omega^{t} \wedge \pi_{i t} \quad\left(\bmod \quad \varpi_{0}, \ldots, \varpi_{r}\right) \quad \text { for } \quad i=0, \ldots, r,
$$

where $\omega^{\alpha}, \pi_{i \alpha}(1 \leqq i \leqq r, 1 \leqq \alpha \leqq t)$ are 1 -forms defined around $y_{0} \in Y$ such that $\left\{\varpi_{0}, \ldots, \varpi_{r}, \omega^{1}\right.$, $\left.\ldots, \omega^{t}\right\}$ are linearly independent on $U$. late

Moreover, from $d \varpi_{i} \equiv \sum_{\alpha=1}^{t} \omega^{\alpha} \wedge \pi_{i \alpha}+\sum_{j=1}^{r} \gamma_{i}^{j} \wedge \varpi_{j} \quad(\bmod \quad \varpi)$ for $i=0, \ldots, r$, we calcu-

$$
d \varpi \equiv \sum_{\alpha=1}^{t} \omega^{\alpha} \wedge\left(\pi_{0 \alpha}+\sum_{i=1}^{r} \lambda_{i} \pi_{i \alpha}\right)+\sum_{i=1}^{r}\left(d \lambda_{i}+\gamma_{0}^{i}+\sum_{j=1}^{r} \lambda_{j} \gamma_{j}^{i}\right) \wedge \varpi_{i} \quad(\bmod \varpi) .
$$

By the Engel half-rank condition, we may assume $\left(d \varpi_{0}\right)^{t} \not \equiv 0 \quad\left(\bmod \varpi_{0}, \ldots, \varpi_{r}\right)$ as before, i.e., $\left\{\varpi_{0}, \ldots, \varpi_{r}, \omega^{1}, \ldots, \omega^{t}, \pi_{01}, \ldots, \pi_{0 t}\right\}$ are linearly independent around $y_{o} \in Y$. Then, putting $n=r+t, \varpi^{\alpha}=\pi_{0 \alpha}++\sum_{i=1}^{r} \lambda_{i} \pi_{i \alpha}$ and $\Omega_{i}=\gamma_{0}^{i}+\sum_{j=1}^{r} \lambda_{j} \gamma_{j}^{i}$, we see that corank of $\mathrm{Ch}\left(C_{Y}^{*}\right)(w)$ equals $2 n+1$ if and only if $\left\{\varpi^{1}, \ldots, \varpi^{t}\right\}$ are linearly independent $\left(\bmod \varpi, \varpi_{1}, \ldots\right.$, $\left.\varpi_{r}, \omega^{1}, \ldots, \omega^{t}, d \lambda_{1}+\Omega_{1}, \ldots, d \lambda_{r}+\Omega_{r}\right)$ at each point $w$. Hence, on a neighborhood of $w_{0}$ in $W(Y)$, where $\left\{\varpi, \varpi_{i}, d \lambda_{i}, \varpi^{\alpha}, \omega^{\alpha}(1 \leqq i \leqq r, 1 \leqq \alpha \leqq t)\right\}$ are linearly independent at each point, we have

$$
C_{Y}^{*}=\{\varpi=0\}, \quad N_{Y}=\left\{\varpi=\varpi_{1}=\cdots=\varpi_{r}=0\right\},
$$

and

$$
d \varpi \equiv \sum_{\alpha=1}^{t} \omega^{\alpha} \wedge \varpi^{\alpha}+\sum_{i=1}^{r}\left(d \lambda_{i}+\Omega_{i}\right) \wedge \varpi_{i} \quad(\bmod \quad \varpi)
$$

Thus

$$
\operatorname{Ch}\left(C_{Y}^{*}\right)=\left\{\varpi=\varpi_{i}=\varpi^{\alpha}=\omega^{\alpha}=d \lambda_{i}+\Omega_{i}=0 \quad(1 \leqq i \leqq r, 1 \leqq \alpha \leqq t)\right\},
$$

and

$$
N_{Y}^{\perp}=\left\{\varpi=\varpi_{i}=\varpi^{\alpha}=\omega^{\alpha}=0 \quad(1 \leqq i \leqq r, 1 \leqq \alpha \leqq t)\right\} .
$$


Then, from $N_{Y}^{\perp}(w) \subset \hat{v} \subset N_{Y}(w)$ and $\left.d \varpi\right|_{\hat{v}}=0$, if $\left.\omega^{1} \wedge \cdots \wedge \omega^{t}\right|_{\hat{v}} \neq 0$, it follows that

$$
\hat{v}=\left\{X \in N_{Y}(w) \mid\left(\varpi^{\alpha}-\sum_{\beta=1}^{t} p_{\alpha \beta}^{*} \omega^{\beta}\right)(X)=0 \quad(1 \leqq \alpha \leqq t)\right\},
$$

where $p_{\alpha \beta}^{*}=p_{\beta \alpha}^{*}(1 \leqq \alpha, \beta \leqq t)$. For these $\hat{v} \in R_{w}$, we have

Lemma 7.1. $A(\hat{v})=\{0\} \quad$ for $\quad \hat{v}=\left\{\varpi_{0}=\cdots=\varpi_{r}=\varpi^{\alpha}-\sum_{\beta=1}^{t} p_{\alpha \beta}^{*} \omega^{\beta}=0 \quad(1 \leqq \alpha \leqq t)\right\}$.

Proof. First we have

$$
d \varpi_{i} \equiv \omega^{1} \wedge \pi_{i 1}+\cdots+\omega^{t} \wedge \pi_{i t} \quad\left(\bmod \quad \varpi_{0}, \ldots, \varpi_{r}\right) \quad \text { for } \quad i=0, \ldots, r,
$$

Hence, for $X \in S\left(N_{Y}\right)(w)=\operatorname{Ch}\left(C_{Y}^{*}\right)(w)$, we have

$$
X\rfloor d \varpi_{i} \equiv-\sum_{\alpha=1}^{t} \pi_{i \alpha}(X) \omega^{\alpha} \quad\left(\bmod \quad(\hat{v})^{\perp}\right),
$$

for $i=0, \ldots, r$. Then, from Lemma $4.2(2)$, we get

$$
\begin{aligned}
A(\hat{v}) & =\left\{X \in S\left(N_{Y}\right)(w) \mid \pi_{i \alpha}(X)=0 \quad(0 \leqq i \leqq r, 1 \leqq \alpha \leqq t)\right\} \\
& =\operatorname{Ch}\left(C_{Y}^{*}\right)(w) \cap \operatorname{Ch}\left(N_{Y}\right)(w)=\{0\} .
\end{aligned}
$$

Thus we obtain

Proposition 7.3. Let $\left(Y, D_{N}\right)$ be a differential system satisfying $(Y .1)$ and $(Y .3)$.

Then $\left(R(Y) ; D_{Y}^{1}, D_{Y}^{2}\right)$ is a PD manifold of second order on an open subset of $R(Y)$.

Here we note that, for $\hat{v}_{1}=\left\{\varpi_{0}=\cdots=\varpi_{r}=\omega^{\alpha}=0 \quad(1 \leqq \alpha \leqq t)\right\} \in R(Y)$, we have $A\left(\hat{v}_{1}\right)=\operatorname{Ch}\left(C_{Y}^{*}\right)$, which follows from $d \varpi_{i} \equiv 0 \quad\left(\bmod \varpi_{0}, \ldots, \varpi_{r}, \omega^{\alpha}(1 \leqq \alpha \leqq t)\right)$ for $i=0, \ldots, r$. Hence $\left(R(Y) ; D_{Y}^{1}, D_{Y}^{2}\right)$ is not a $P D$ manifold of second order globally in this case.

When $\left(R(Y) ; D_{Y}^{1}, D_{Y}^{2}\right)$ becomes a $P D$ manifold of second order satisfying the compatibility condition $(C), \hat{N}_{Y}$ defines a subspace $\pi_{-2}\left(\hat{N}_{Y}(\hat{v})\right)$ of $\mathfrak{s}_{-2}(\hat{v})=D_{Y}^{1}(\hat{v}) / D_{Y}^{2}(\hat{v})$ at each point $\hat{v} \in R(Y)$, where $\pi_{-2}: D_{Y}^{1}(\hat{v}) \rightarrow \mathfrak{s}_{-2}(\hat{v})$ is the projection. This subspace defines the subspace $E(\hat{v})^{\perp}$ of $V(\hat{v})^{*}$, through the symbol algebra identifications of $\left(R(Y) ; D_{Y}^{1}, D_{Y}^{2}\right)$ at $\hat{v} \in R(Y)$ : $\mathfrak{s}_{-2}(\hat{v}) \cong V(\hat{v})^{*}, \mathfrak{s}_{-1}=V(\hat{v}) \oplus \mathfrak{f}(\hat{v})$ and $\mathfrak{f}(\hat{v}) \subset S^{2}\left(V(\hat{v})^{*}\right)$, where $V(\hat{v})$ is an integral element of $\left(R(Y), D_{Y}^{2}\right)$ at $\hat{v}$. Thus we obtain the subspace $E(\hat{v}) \subset V(\hat{v})$. Then, by the construction of $R(Y)$ in $\S 6.1$, we have $\mathfrak{f}(\hat{v})^{\perp} \subset E(\hat{v}) \otimes_{S} V(\hat{v})$. Moreover we have $\hat{E}(\hat{v})=E(\hat{v})$, which follows from Proposition $7.1(2)$. Thus $E(\hat{v})$ satisfies $S^{2}(E(\hat{v})) \subset \mathfrak{f}(\hat{v})^{\perp} \subset E(\hat{v}) \otimes_{S} V(\hat{v})$. Hence $E(\hat{v})$ is $G_{0}(\mathfrak{s}(\hat{v}))$-invariant. Thus $\hat{N}_{Y}$ is a (first order) covariant system of $\left(R(Y) ; D_{Y}^{1}, D_{Y}^{2}\right)$.

7.2. General Case. Now, starting from a pair of differential systems $\left(Y ; D_{N}^{*}, D_{N}\right)$, we will construct an $I G$ manifold $\left(W(Y) ; C_{Y}^{*}, N_{Y}\right)$ over $\left(Y ; D_{N}^{*}, D_{N}\right)$ such that $N_{Y}=\mu^{-1}\left(D_{N}\right)$ and $\bar{N}_{Y}=\mu^{-1}\left(D_{N}^{*}\right)$ where $\mu: W(Y) \rightarrow Y$ is the projection, and the Lagrange Grassmann bundle $R(Y)=R(W(Y))$ over $\left(W(Y) ; C_{Y}^{*}, N_{Y}\right)$ and will examine the conditions for $\bar{N}_{Y}=N_{Y}^{*}$ and when $\left(R(Y) ; D_{Y}^{1}, D_{Y}^{2}\right)$ becomes a $P D$ manifold of second order.

Let $\left(Y ; D_{N}^{*}, D_{N}\right)$ be a pair of differential systems on $Y$ satisfying the following condition;

$(\hat{Y} .1) \quad D_{N}^{*}$ and $D_{N}$ are differential systems of codimension $s+1$ and $r+1$ on $Y$ such that

$$
D_{N}^{*} \supset D_{N} \supset \mathrm{Ch}\left(D_{N}^{*}\right) \supset \mathrm{Ch}\left(D_{N}\right)=\{0\} .
$$

We assume that $D_{N}^{*}$ is of constant Engel half-rank and let $t_{1}$ be the Engel half-rank of $D_{N}^{*}$. Let 
$\left\{\varpi_{0}, \ldots, \varpi_{s}\right\}$ be a local defining 1 -forms of $\left(Y, D_{N}^{*}\right)$ on a neighborhood $U$ of $y_{o} \in Y$. Then, for a section $\varpi \in \Gamma\left(\left(D_{N}^{*}\right)^{\perp}\right)$, we have

$$
(d \varpi)^{t_{1}+1} \equiv 0 \quad\left(\bmod \quad \varpi_{0}, \ldots, \varpi_{s}\right),
$$

on $U$ and $(d \hat{\varpi})^{t_{1}} \not \equiv 0$ for some $\hat{\varpi} \in \Gamma\left(\left(D_{N}^{*}\right)^{\perp}\right)$. Here we may assume $\left(d \varpi_{0}\right)^{t_{1}} \not \equiv 0$ around $y_{o} \in Y$.

Now let us consider the collection $\tilde{W}(Y)$ of hyperplanes $w$ in each tangent space $T_{y}(Y)$ at $y \in Y$ which contains the fibre $D_{N}^{*}(y)$ of $D_{N}^{*}$;

$$
\begin{gathered}
\tilde{W}(Y)=\bigcup_{y \in Y} \tilde{W}_{y} \subset J(Y, m-1), \\
\tilde{W}_{y}=\left\{w \in \operatorname{Gr}\left(T_{y}(Y), m-1\right) \mid w \supset D_{N}^{*}(y)\right\} \cong \mathbb{P}\left(T_{y}(Y) / D_{N}^{*}(y)\right)=\mathbb{P}^{s},
\end{gathered}
$$

where $m=\operatorname{dim} Y$. Moreover $C_{Y}^{*}$ is the canonical system obtained by the Grassmannian construction, $\bar{N}_{Y}$ and $N_{Y}$ are the lifts of $D_{N}^{*}$ and $D_{N}$ respectively. Precisely, $C_{Y}^{*}, \bar{N}_{Y}$ and $N_{Y}$ are given by

$$
C_{Y}^{*}(w)=\mu_{*}^{-1}(w) \supset \bar{N}_{Y}(w)=\mu_{*}^{-1}\left(D_{N}^{*}(y)\right) \supset N_{Y}(w)=\mu_{*}^{-1}\left(D_{N}(y)\right),
$$

for each $w \in \tilde{W}(Y)$ and $y=\mu(w)$, where $\mu: \tilde{W}(Y) \rightarrow Y$ is the projection.

We will now examine the condition for $\left(\tilde{W}(Y) ; C_{Y}^{*}, N_{Y}\right)$ to be an $I G$ manifold of corank $r$. Let us consider

$$
\varpi=\varpi_{0}+\lambda_{1} \varpi_{1}+\cdots+\lambda_{s} \varpi_{s}
$$

on $U$. Namely we consider a point $w \in \tilde{W}(Y)$ such that $w=\{\varpi=0\} \subset T_{y}(Y)$, where $y=\mu(w) \in U$. Here $\left(\lambda_{1}, \ldots, \lambda_{s}\right)$ constitutes an inhomogeneous coordinate of the fibres of $\mu: \hat{W}(Y) \rightarrow Y$. Denoting the pullback on $\tilde{W}(Y)$ of 1 -forms on $Y$ by the same symbol, we have

$$
C_{Y}^{*}=\{\varpi=0\},
$$

and

$$
d \varpi=d \varpi_{0}+\sum_{i=1}^{s} \lambda_{i} d \varpi_{i}+\sum_{i=1}^{s} d \lambda_{i} \wedge \varpi_{i} .
$$

on $\mu^{-1}(U)$. Then, as in $\S 7.1$, by the Engel half-rank condition, we see that the following subset $W(Y)$ of $\tilde{W}(Y)$ is an open (dense) subset of $\tilde{W}(Y)$;

$$
W(Y)=\left\{w \in \tilde{W}(Y) \mid \operatorname{corank} \operatorname{Ch}\left(C_{Y}^{*}\right)(w)=2 n+1\right\}
$$

where $n=s+t_{1}$. Assume that $\left(W(Y) ; C_{Y}^{*}, N_{Y}\right)$ is an $I G$ manifold of corank $r$. Then, by the condition (W.3) in $\S 2.2$, we have $\left.\operatorname{rank} d \varpi\right|_{N_{Y}(w)}=2(n-r)$ at each $w \in W(Y)$. Namely we have, at each point $w=\left\{\varpi=\varpi_{0}+\sum_{i=1}^{s} \lambda_{i} \varpi_{i}=0\right\} \in W(Y)$,

$$
d \varpi \equiv d \varpi_{0}+\sum_{i=1}^{s} \lambda_{i} d \varpi_{i} \equiv \sum_{\alpha=1}^{n-r} \tilde{\omega}^{\alpha} \wedge \tilde{\varpi}^{\alpha} \quad\left(\bmod \quad \varpi_{0}, \ldots, \varpi_{r}\right),
$$

where $D_{N}^{*}=\left\{\varpi_{0}=\cdots=\varpi_{s}=0\right\}$ and $D_{N}=\left\{\varpi_{0}=\cdots=\varpi_{r}=0\right\}$. Thus $\left(Y ; D_{N}^{*}, D_{N}\right)$ satisfies the following condition;

$(\hat{Y} .2) \quad$ The Engel half-rank of $D_{N}^{*}\left(\bmod \quad D_{N}^{\perp}\right)$ equals to $t_{2}=t_{1}-(r-s)=n-r$.

Conversely, under this condition, there exists a local defining 1 -forms $\left\{\varpi_{0}, \ldots, \varpi_{r}\right\}$ of $\left(Y, D_{N}\right)$ on a neighborhood $U$ of $y_{o} \in Y$ such that

$$
D_{N}^{*}=\left\{\varpi_{0}=\cdots=\varpi_{s}=0\right\}, \quad D_{N}=\left\{\varpi_{0}=\cdots=\varpi_{r}=0\right\},
$$


and

$$
\begin{aligned}
d \varpi_{0}+\sum_{i=1}^{s} \lambda_{i} d \varpi_{i} & \equiv \sum_{\alpha=1}^{t_{2}} \tilde{\omega}^{\alpha} \wedge \tilde{\varpi}^{\alpha} \quad\left(\bmod \quad \varpi_{0}, \ldots, \varpi_{r}\right), \\
& \equiv \sum_{j=s+1}^{r} \omega_{j} \wedge \varpi_{j}+\sum_{\alpha=1}^{t_{2}} \tilde{\omega}^{\alpha} \wedge \tilde{\varpi}^{\alpha} \quad\left(\bmod \quad \varpi_{0}, \ldots, \varpi_{s}\right),
\end{aligned}
$$

where $\tilde{\omega}^{\alpha}, \tilde{\varpi}^{\alpha}\left(1 \leqq \alpha \leqq t_{2}\right)$ and $\omega_{j}(s+1 \leqq j \leqq r)$ are 1 -forms on U defined around $y_{0}$ such that $\left\{\varpi_{0}, \ldots, \varpi_{r}, \tilde{\omega}^{\alpha}, \tilde{\varpi}^{\alpha}\left(1 \leqq \alpha \leqq t_{2}\right)\right\}$ are linearly independent at each point. Moreover, by the Engel half-rank condition for $D_{N}^{*}$, we see that $\left\{\varpi_{0}, \ldots, \varpi_{r}, \omega_{j}(s+1 \leqq j \leqq r), \tilde{\omega}^{\alpha}, \tilde{\varpi}^{\alpha}(1 \leqq \alpha \leqq\right.$ $\left.\left.t_{2}\right)\right\}$ are linearly independent around $y_{0} \in Y$.

Now we claim

Proposition 7.4. Let $\left(Y ; D_{N}^{*}, D_{N}\right)$ be a pair of differential systems satisfying $(\hat{Y} .1)$ and $(\hat{Y} .2)$ and let $t_{1}$ be the Engel half-rank of $D_{N}^{*}$. Then $\left(W(Y) ; C_{Y}^{*}, N_{Y}\right)$ is an $I G$ manifold of corank $r$ , where $n=r+t_{2}=s+t_{1}$. Moreover

$$
\bar{N}_{Y} \supset N_{Y}^{*}=\partial N_{Y}^{\perp}+N_{Y} \quad \text { and } \quad N_{Y} \supset \partial \bar{N}_{Y}^{\perp} .
$$

Proof. Notations being as above, putting

$$
d \varpi_{0}+\sum_{i=1}^{s} \lambda_{i} d \varpi_{i} \equiv \sum_{a=1}^{s} \gamma_{a} \wedge \varpi_{a}+\sum_{j=s+1}^{r} \omega_{j} \wedge \varpi_{j}+\sum_{\alpha=1}^{t_{2}} \tilde{\omega}^{\alpha} \wedge \tilde{\varpi}^{\alpha} \quad(\bmod \varpi),
$$

we get

$$
d \varpi \equiv \sum_{a=1}^{s}\left(d \lambda_{a}+\gamma_{a}\right) \wedge \varpi_{a}+\sum_{j=s+1}^{r} \omega_{j} \wedge \varpi_{j}+\sum_{\alpha=1}^{t_{2}} \tilde{\omega}^{\alpha} \wedge \tilde{\varpi}^{\alpha} \quad(\bmod \varpi),
$$

on a neiborghhood of $w_{0}=\left\{\varpi_{0}=0\right\} \in W(Y)$. Thus we have

$$
C_{Y}^{*}=\{\varpi=0\}, \quad \bar{N}_{Y}=\left\{\varpi=\varpi_{1}=\cdots=\varpi_{s}=0\right\}, \quad N_{Y}=\left\{\varpi=\varpi_{1}=\cdots=\varpi_{r}=0\right\},
$$

$\operatorname{Ch}\left(C_{Y}^{*}\right)=\left\{\varpi=\varpi_{a}=\varpi_{j}=\tilde{\varpi}^{\alpha}=\tilde{\omega}^{\alpha}=\omega_{j}=d \lambda_{a}+\gamma_{a}=0 \quad\left(1 \leqq a \leqq s, s+1 \leqq j \leqq r, 1 \leqq \alpha \leqq t_{2}\right)\right\}$. and

$$
\begin{gathered}
N_{Y}^{\perp}=\left\{\varpi=\varpi_{1}=\cdots=\varpi_{r}=\tilde{\varpi}^{\alpha}=\tilde{\omega}^{\alpha}=0 \quad\left(1 \leqq \alpha \leqq t_{2}\right)\right\}, \\
\bar{N}_{Y}^{\perp}=\left\{\varpi=\varpi_{1}=\cdots=\varpi_{r}=\tilde{\varpi}^{\alpha}=\tilde{\omega}^{\alpha}=\omega_{j}=0 \quad\left(1 \leqq \alpha \leqq t_{2}, s+1 \leqq j \leqq r\right)\right\} .
\end{gathered}
$$

Moreover, from $\operatorname{Ch}\left(D_{N}\right)=\{0\}$, we have $\operatorname{Ch}\left(N_{Y}\right)=\operatorname{Ker} \mu_{*}$, which implies $\operatorname{Ch}\left(C_{Y}^{*}\right) \cap \operatorname{Ch}\left(N_{Y}\right)=$ $\{0\}$. Thus $\left(W(Y) ; C_{Y}^{*}, N_{Y}\right)$ is an $I G$ manifold of corank $r$.

$\bar{N}_{Y} \supset \partial N_{Y}^{\perp}$ follows from

$$
d \varpi_{a} \equiv 0 \quad\left(\bmod \quad \varpi_{0}, \ldots, \varpi_{r}, \tilde{\varpi}^{\alpha}, \tilde{\omega}^{\alpha} \quad\left(1 \leqq \alpha \leqq t_{2}\right)\right),
$$

for $a=0, \ldots, s$, which follows from (7.2) and [4] II Proposition 4.1.

Moreover, from $d \varpi \equiv \sum_{j=s+1}^{r} \omega_{j} \wedge \varpi_{j}+\sum_{\alpha=1}^{t_{2}} \tilde{\omega}^{\alpha} \wedge \tilde{\varpi}^{\alpha} \quad\left(\bmod \quad \varpi_{0}, \ldots, \varpi_{s}\right)$, we have $\bar{N}_{Y}^{\perp} \supset$ $\mathrm{Ch}\left(\bar{N}_{Y}\right) \supset \mathrm{Ch}\left(N_{Y}\right)$. Hence, by rank comparison, we get $\bar{N}_{Y}^{\perp}=\mathrm{Ch}\left(N_{Y}\right) \oplus \mathrm{Ch}\left(C_{Y}^{*}\right)$, which implies that $N_{Y} \supset \partial \bar{N}_{Y}^{\perp}$.

As for the criteria to the condition $\bar{N}_{Y}=N_{Y}^{*}$, utilizing Proposition 2.1 in $\S 2.3$ (3), we add the following ;

Proposition 7.5. Let $\left(Y ; D_{N}^{*}, D_{N}\right)$ be a pair of differential systems satisfying $(\hat{Y} .1)$ and $(\hat{Y} .2)$. Assume that $\left(W(Y) ; C_{Y}^{*}, N_{Y}\right)$ satisfies the compatibility condition $\left(C^{*}\right)$. Then $\bar{N}_{Y}=N_{Y}^{*}$ holds if and only if $H\left(N_{Y}\right)=C h\left(N_{Y}\right) \oplus S\left(N_{Y}\right)$. 
Proof. By Proposition 7.4, we have $\bar{N}_{Y} \supset N_{Y}^{*}$. Hence $\bar{N}_{Y}=N_{Y}^{*}$ if and only if $\operatorname{dim} C_{Y}^{*}(w) / N_{Y}^{*}(w)=$ $s$ at each $w \in W(Y)$. By Proposition 2.1, we have $\operatorname{dim} C_{Y}^{*}(w) / N_{Y}^{*}(w)=\operatorname{dim} H\left(N_{Y}\right)(w) / S\left(N_{Y}\right)(w)$. On the other hand, $H\left(N_{Y}\right) \supset \mathrm{Ch}\left(N_{Y}\right) \oplus S\left(N_{Y}\right)$ and $\operatorname{rank} \mathrm{Ch}\left(N_{Y}\right)=s$. Hence $\bar{N}_{Y}=N_{Y}^{*}$ holds if and only if $H\left(N_{Y}\right)=\mathrm{Ch}\left(N_{Y}\right) \oplus S\left(N_{Y}\right)$.

Now we consider the Lagrange Grassmann bundle $R(Y)=R(W(Y))$ over $\left(W(Y) ; C_{Y}^{*}, N_{Y}\right)$ :

$$
R(Y)=\bigcup_{w \in W(Y)} R_{w}, \quad R_{w}=\left\{\hat{v} \subset N_{Y}(w)\left|\gamma_{w}\right|_{\hat{v}}=0, \quad \hat{v} \text { is maximal }\right\} .
$$

Let $D_{Y}^{2}$ be the canonical system on $R(Y)$ and let $D_{Y}^{1}$ and $\hat{N}_{Y}$ be the lifts of $C_{Y}^{*}$ and $N_{Y}$ respectively, i.e.,

$$
D_{Y}^{1}(\hat{v})=\tau_{*}^{-1}\left(C_{Y}^{*}(w)\right), \quad D_{Y}^{2}(\hat{v})=\tau_{*}^{-1}(\hat{v}), \quad \hat{N}_{Y}(\hat{v})=\tau_{*}^{-1}\left(N_{Y}(w)\right),
$$

where $\tau: R(Y) \rightarrow W(Y)$ is the projection and $w=\tau(\hat{v})$.

In order to see whether $\left(R(Y) ; D_{Y}^{1}, D_{Y}^{2}\right)$ is a $P D$ manifold of second order, we must check the condition $A(\hat{v})=\{0\}$ for each $\hat{v} \in R(Y)$, utilizing, e.g., Lemma 4.2 together with the structure equation of $D_{N}$. Assuming the conditions $(\hat{Y} .1)$ and $(\hat{Y} .2)$, by Proposition 7.4, we see that the structure quation of $N_{Y}$ takes the following form;

$$
\left\{\begin{array}{cl}
d \varpi \equiv & \sum_{\alpha=1}^{t_{2}} \tilde{\omega}^{\alpha} \wedge \tilde{\varpi}_{\alpha}, \\
d \varpi_{a} \equiv \quad & \sum_{\alpha=1}^{t_{2}} \tilde{\omega}^{\alpha} \wedge \pi_{a \alpha}+\sum_{\alpha=1}^{t_{2}} \tilde{\varpi}^{\alpha} \wedge \hat{\pi}_{a \alpha} \quad(1 \leqq a \leqq s), \quad\left(\bmod \varpi, \varpi_{1}, \ldots, \varpi_{r}\right) \\
d \varpi_{i} \equiv \sum_{j=s+1}^{r} \omega^{j} \wedge \pi_{i j}+\sum_{\alpha=1}^{t_{2}} \tilde{\omega}^{\alpha} \wedge \pi_{i \alpha}+\sum_{\alpha=1}^{t_{2}} \tilde{\varpi}^{\alpha} \wedge \hat{\pi}_{i \alpha} \quad(s+1 \leqq i \leqq r),
\end{array}\right.
$$

Here, by Lemma 4.2 (2), we note

$$
\begin{gathered}
S\left(N_{Y}\right)(w)=\left\{X \in \operatorname{Ch}\left(C_{Y}^{*}\right)(w) \mid \pi_{i j}(X)=0(s+1 \leqq i, j \leqq r)\right\}, \\
A\left(\hat{v}_{1}\right)=\left\{X \in S\left(N_{Y}\right)(w) \mid\left(\pi_{a \alpha}+\sum_{\beta=1}^{t_{2}} p_{\alpha \beta} \hat{\pi}_{a \beta}\right)(X)=\right. \\
\left.\left(\pi_{i \alpha}+\sum_{\beta=1}^{t_{2}} p_{\alpha \beta} \hat{\pi}_{i \beta}\right)(X)=0\left(1 \leqq a \leqq s, s+1 \leqq i \leqq r, 1 \leqq \alpha \leqq t_{2}\right)\right\}
\end{gathered}
$$

for $\hat{v}_{1}=\left\{\varpi=\varpi_{1}=\cdots=\varpi_{r}=\tilde{\varpi}^{\alpha}-\sum_{\beta=1}^{t_{2}} p_{\alpha \beta} \tilde{\omega}^{\beta}=0\left(1 \leqq \alpha \leqq t_{2}\right)\right\} \in R(Y)$ and

$$
\begin{aligned}
A\left(\hat{v}_{2}\right)=\left\{X \in S\left(N_{Y}\right)(w) \mid\right. & \left(\hat{\pi}_{a \alpha}+\sum_{\beta=1}^{t_{2}} p_{\alpha \beta} \pi_{a \beta}\right)(X)= \\
& \left.\left(\hat{\pi}_{i \alpha}+\sum_{\beta=1}^{t_{2}} p_{\alpha \beta} \pi_{i \beta}\right)(X)=0\left(1 \leqq a \leqq s, s+1 \leqq i \leqq r, 1 \leqq \alpha \leqq t_{2}\right)\right\}
\end{aligned}
$$

for $\hat{v}_{2}=\left\{\varpi=\varpi_{1}=\cdots=\varpi_{r}=\tilde{\omega}^{\alpha}-\sum_{\beta=1}^{t_{2}} p_{\alpha \beta} \tilde{\varpi}^{\beta}=0\left(1 \leqq \alpha \leqq t_{2}\right)\right\} \in R(Y)$. Thus we need some more information on the structure of $D_{N}$ to conclude $A\left(\hat{v}_{1}\right)=\{0\}$ or $A\left(\hat{v}_{2}\right)=\{0\}$. We will examine several examples of this case in $\S 8.2$. 


\section{Examples of Second Reduction Theorem}

8.1. Case $n=3$. In [6], for involutive systems of second order partial differential equations for a scalar function with 3 independent variables, E.Cartan first classified involutive subspaces $\mathfrak{f}$ of $S^{2}\left(V^{*}\right)$, over the complex number field $\mathbb{C}$, when $\operatorname{dim} V=3$. In this subsection, following his classification, we will here indicate $G_{0}(\mathfrak{s})$-invariant subspaces $E$ and $\hat{E}$, satisfying $\mathfrak{f}^{\perp} \subset E \otimes_{S} V$, for each involutive subspace $\mathfrak{f} \subset S^{2}\left(V^{*}\right)$, when $\operatorname{dim} V=3$, which shows the applicability of Theorem 5.1 or 5.2 in each case.

(1) $\operatorname{codim} \mathfrak{f}=1$.

In this case $\operatorname{dim} \mathfrak{f}^{\perp}=1$. Hence we can classify a generator $f$ of $\mathfrak{f}^{\perp}$ as a quadratic form and obtain the following classification by the rank of $f$ into three cases over $\mathbb{C}$, i.e., there exista a basis $\left\{e_{1}, e_{2}, e_{3}\right\}$ of $V$ such that

$$
\mathfrak{f}^{\perp}=\left\langle\left\{e_{1} \odot e_{1}+e_{2} \odot e_{2}+e_{3} \odot e_{3}\right\}\right\rangle, \quad\left\langle\left\{e_{1} \odot e_{2}\right\}\right\rangle, \quad \text { or } \quad\left\langle\left\{e_{1} \odot e_{1}\right\}\right\rangle .
$$

In the first case, we have no $G_{0}(\mathfrak{s})$-invariant subspace. In the second case, $E=\left\langle\left\{e_{1}, e_{2}\right\}\right\rangle$, $\left\langle\left\{e_{1}\right\}\right\rangle$ or $\left\langle\left\{e_{2}\right\}\right\rangle$ and $\hat{E}=\{0\}$ in either case. In the third case, $E=\hat{E}=\left\langle\left\{e_{1}\right\}\right\rangle$. The third case corrresponds to the Goursat type equation (see Theorem 6.1 (1)).

(2) $\operatorname{codim} \mathfrak{f}=2$.

In this case, there exists a basis $\left\{e_{1}, e_{2}, e_{3}\right\}$ of $V$ such that (see Proposition 3.1 [27])

$$
\mathfrak{f}^{\perp}=\left\langle\left\{e_{1} \odot e_{2}, e_{1} \odot e_{3}\right\}\right\rangle, \quad \text { or }\left\langle\left\{e_{1} \odot e_{1}, e_{1} \odot e_{2}\right\}\right\rangle .
$$

In the first case, $E=\left\langle\left\{e_{2}, e_{3}\right\}\right\rangle$ or $\left\langle\left\{e_{1}\right\}\right\rangle$ and $\hat{E}=\{0\}$ in either case. In the second case, $E_{1}=\hat{E}_{1}=\left\langle\left\{e_{1}\right\}\right\rangle$ or $E_{2}=\left\langle\left\{e_{1}, e_{2}\right\}\right\rangle$ and $\hat{E}_{2}=\left\langle\left\{e_{1}\right\}\right\rangle$ (see $\S 8.3$ for $E_{2}$-case).

In fact, E.Cartan showed $C(E)$ is completely integrable for $E=\left\langle\left\{e_{1}\right\}\right\rangle$ in the first case so that Theorem 5.1 is applicable. In the second case, he showed $C\left(E_{2}\right)$ is completely integrable in case of $\left(b_{1}\right)$ and $C\left(E_{1}\right)$ is completely integrable in case of $\left(b_{2}\right)$ and $\left(b_{3}\right)$ so that Theorem 5.2 is applicable (cf. [21]).

(3) $\operatorname{codim} \mathfrak{f}=3$.

In this case, there exists a basis $\left\{e_{1}, e_{2}, e_{3}\right\}$ of $V$ such that (see IV [6])

$$
\begin{gathered}
\mathfrak{f}^{\perp}=\left\langle\left\{e_{1} \odot e_{2}, e_{1} \odot e_{3}, e_{2} \odot e_{3}\right\}\right\rangle, \quad\left\langle\left\{e_{1} \odot e_{1}, e_{1} \odot e_{3}, e_{2} \odot e_{3}\right\}\right\rangle, \quad\left\langle\left\{e_{1} \odot e_{1}, e_{1} \odot e_{2}, e_{2} \odot e_{2}-e_{1} \odot e_{3}\right\}\right\rangle, \\
\left\langle\left\{e_{1} \odot e_{1}, e_{1} \odot e_{2}, e_{2} \odot e_{2}\right\}\right\rangle, \quad \text { or } \quad\left\langle\left\{e_{1} \odot e_{1}, e_{1} \odot e_{2}, e_{1} \odot e_{3}\right\}\right\rangle .
\end{gathered}
$$

In the first case, $E=\left\langle\left\{e_{1}, e_{2}\right\}\right\rangle,\left\langle\left\{e_{1}, e_{3}\right\}\right\rangle$ or $\left\langle\left\{e_{2}, e_{3}\right\}\right\rangle$ and $\hat{E}=\{0\}$ in either case. In the second case, $E=\left\langle\left\{e_{1}, e_{2}\right\}\right\rangle$ or $\left\langle\left\{e_{1}, e_{3}\right\}\right\rangle$ and $\hat{E}=\left\langle\left\{e_{1}\right\}\right\rangle$ in either case. In the third case, $E=\left\langle\left\{e_{1}, e_{2}\right\}\right\rangle$ and $\hat{E}=\left\langle\left\{e_{1}\right\}\right\rangle$. In [6], the case when $C(E)$ is completely integrable, has been discussed in detail. In the fourth case, $E=\hat{E}=\left\langle\left\{e_{1}, e_{2}\right\}\right\rangle$ and $\mathfrak{f} \cong \mathfrak{f}^{2}(2)$ (see Theorem $6.2(2)$ ). In the fifth case, $E=\hat{E}=\left\langle\left\{e_{1}\right\}\right\rangle$ corresponds to the Cauchy characteristics and $\mathfrak{f} \cong \mathfrak{f}^{3}(1)$ (see $\S 6.1[27])$.

(4) $\operatorname{codim} \mathfrak{f}=4$.

In this case, there exists a basis $\left\{e_{1}, e_{2}, e_{3}\right\}$ of $V$ such that (see $\mathrm{V}[6]$ )

$$
\mathfrak{f}^{\perp}=\left\langle\left\{e_{1} \odot e_{1}, e_{1} \odot e_{2}, e_{1} \odot e_{3}, e_{2} \odot e_{3}\right\}\right\rangle, \quad \text { or } \quad\left\langle\left\{e_{1} \odot e_{1}, e_{1} \odot e_{2}, e_{2} \odot e_{2}, e_{1} \odot e_{3}\right\}\right\rangle .
$$

In the first case, $E=\left\langle\left\{e_{1}, e_{2}\right\}\right\rangle$ or $\left\langle\left\{e_{1}, e_{3}\right\}\right\rangle$ and $\hat{E}=\left\langle\left\{e_{1}\right\}\right\rangle$ in either case. $\hat{E}$ corresponds to the Cauchy characteristics. Hence Theorem 5.1 or 5.2 is not applicable to this case. In the second case, $E=\hat{E}=\left\langle\left\{e_{1}, e_{2}\right\}\right\rangle$ and $\left\langle\left\{e_{1}\right\}\right\rangle$ corresponds to the Cauchy characteristics (see $\S 8.2)$.

(5) $\operatorname{codim} \mathfrak{f}=5$. 
In this case, there exists a basis $\left\{e_{1}, e_{2}, e_{3}\right\}$ of $V$ such that (see VI [6])

$$
\mathfrak{f}^{\perp}=\left\langle\left\{e_{1} \odot e_{1}, e_{1} \odot e_{2}, e_{1} \odot e_{3}, e_{2} \odot e_{2}, e_{2} \odot e_{3}\right\}\right\rangle .
$$

In this case, $E=\hat{E}=\left\langle\left\{e_{1}, e_{2}\right\}\right\rangle, \mathfrak{f} \cong \mathfrak{f}^{3}(2)$ and $\operatorname{rank} D^{2}=4$. $E$ corresponds to the Cauchy characteristics. Hence, by the First Reduction Theorem, this case is reduced to the geometry of $(X, D)$, where $\operatorname{rank} D=2$ and $\operatorname{dim} X=6$.

8.2. Case $N^{*}=N$. We exhibit here several examples of simple graded Lie algebras $\mathfrak{g}=$ $\bigoplus_{p \in \mathbb{Z}} \mathfrak{g}_{p}$ of depth 2 , such that we can construct a $P D$ manifold $R(Y)$ of second order from a regular differential system $\left(Y, D_{N}\right)$ of type $\mathfrak{m}$, through the Second Reduction Theorem, where $\mathfrak{m}=\mathfrak{g}_{-2} \oplus \mathfrak{g}_{-1}$ is the negative part of $\mathfrak{g}=\bigoplus_{p=-2}^{2} \mathfrak{g}_{p}$.

The first example is of type $\left(B_{3},\left\{\alpha_{3}\right\}\right)$. The standard differential system $\left(M(\mathfrak{m}), D_{\mathfrak{m}}\right)$ of type $\mathfrak{m}$ in this case is given as follows; $M(\mathfrak{m})=\mathbb{R}^{6}$ is endowed with a coordinate $\left(x_{1}, x_{2}, x_{3}, y_{1}, y_{2}, y_{3}\right)$ such that $D_{\mathfrak{m}}$ is given by

$$
D_{\mathfrak{m}}=\left\{\bar{\theta}_{1}=\bar{\theta}_{2}=\bar{\theta}_{3}=0\right\}
$$

where

$$
\bar{\theta}_{1}=d y_{1}-x_{2} d x_{3}, \bar{\theta}_{2}=d y_{2}-x_{3} d x_{1} \quad \text { and } \quad \bar{\theta}_{3}=d y_{3}-x_{1} d x_{2} .
$$

Thus the symbol algebra $\mathfrak{m}=\mathfrak{g}_{-2} \oplus \mathfrak{g}_{-1}$ of type $\left(B_{3},\left\{\alpha_{3}\right\}\right)$ is described by

$$
\left\{\begin{aligned}
d \theta_{1} & \equiv \omega_{3} \wedge \omega_{2} \\
d \theta_{2} & \equiv \omega_{1} \wedge \omega_{3} \\
d \theta_{3} & \equiv \omega_{2} \wedge \omega_{1}
\end{aligned} \quad\left(\bmod \quad \theta_{1}, \theta_{2}, \theta_{3}\right)\right.
$$

i.e., $\mathfrak{g}_{-2}=\wedge^{2} V$ for $\mathfrak{g}_{-1}=V$, where $\operatorname{dim} V=3$. Namely $\mathfrak{m}=\mathfrak{g}_{-2} \oplus \mathfrak{g}_{-1}$ is the free Lie algebra of the second kind (cf. $\S 5.3[15]$, p.245 [24]). Let $\left(Y, D_{N}\right)$ be a regular differential system of type $\mathfrak{m}$ such that $D_{N}$ is locally defined by

$$
D_{N}=\left\{\theta_{1}=\theta_{2}=\theta_{3}=0\right\} .
$$

Here $\left\{\theta_{1}, \theta_{2}, \theta_{3}, \omega_{1}, \omega_{2}, \omega_{3}\right\}$ forms a coframe on $Y$ satisfying (8.1). Then, putting $\varpi=\theta_{1}+\lambda_{1} \theta_{2}+$ $\lambda_{2} \theta_{3}$, we calculate

$$
\begin{aligned}
d \varpi & \equiv \omega_{3} \wedge \omega_{2}+\lambda_{1} \omega_{1} \wedge \omega_{3}+\lambda_{2} \omega_{2} \wedge \omega_{1}+\left(d \lambda_{1}+\gamma_{1}\right) \wedge \theta_{2}+\left(d \lambda_{2}+\gamma_{2}\right) \wedge \theta_{3} \\
& \equiv\left(\omega_{3}-\lambda_{2} \omega_{1}\right) \wedge\left(\omega_{2}-\lambda_{1} \omega_{1}\right)+\left(d \lambda_{1}+\gamma_{1}\right) \wedge \theta_{2}+\left(d \lambda_{2}+\gamma_{2}\right) \wedge \theta_{3} \\
& \equiv \tilde{\omega}_{3} \wedge \tilde{\omega}_{2}+\left(d \lambda_{1}+\gamma_{1}\right) \wedge \theta_{2}+\left(d \lambda_{2}+\gamma_{2}\right) \wedge \theta_{3} \quad(\bmod \quad \varpi)
\end{aligned}
$$

for some 1 -forms $\gamma_{1}, \gamma_{2}$ on $Y$, where we put $\tilde{\omega}_{2}=\omega_{2}-\lambda_{1} \omega_{1}$ and $\tilde{\omega}_{3}=\omega_{3}-\lambda_{2} \omega_{1}$. Thus, by symmetry in the indices $1,2,3$ in $(8.1)$, we see

$$
W(Y)=\hat{W}(Y)=\bigcup_{y \in Y} \tilde{W}_{y}, \quad \tilde{W}_{y}=\left\{w \in \operatorname{Gr}\left(T_{y}(Y), 5\right) \mid w \supset D_{N}(y)\right\} \cong \mathbb{P}\left(T_{y}(Y) / D_{N}(y)\right)=\mathbb{P}^{2}
$$

and we have on $W(Y)$,

$$
C_{Y}^{*}=\{\varpi=o\}, \quad N_{Y}=\left\{\varpi=\theta_{2}=\theta_{3}=o\right\}, \quad N_{Y}^{\perp}=\left\{\varpi=\theta_{2}=\theta_{3}=\tilde{\omega}_{2}=\tilde{\omega}_{3}=o\right\} .
$$

Here $r=2, t=1, n=r+t=3$ and $\operatorname{dim} W(Y)=8$. Moreover, for the Lagrange Grassmann bundle;

$$
R(Y)=\bigcup_{w \in W(Y)} R_{w}, \quad R_{w}=\left\{\hat{v} \subset N_{Y}(w)|d \varpi|_{\hat{v}}=0, \quad \hat{v} \text { is maximal }\right\}
$$

we have

$$
\hat{v}=\left\{\varpi=\theta_{2}=\theta_{3}=\tilde{\omega}_{3}-p \tilde{\omega}_{2}=0\right\} \text { or }\left\{\varpi=\theta_{2}=\theta_{3}=\tilde{\omega}_{2}-q \tilde{\omega}_{3}=0\right\} .
$$


Then, by Lemma $4.2(2)$ and $(8.1)$, we get $A(\hat{v})=\{0\}$ in any case. Hence $\left(R(Y) ; D_{Y}^{1}, D_{Y}^{2}\right)$ is globally a $P D$ manifold of second order, when $\left(Y, D_{N}\right)$ is a regular differential system of type $\mathfrak{m}$, where $\mathfrak{m}$ is the negative part of the simple graded Lie algebra of type $\left(B_{3},\left\{\alpha_{3}\right\}\right)$.

Here we note : In case $Y=G / P_{3} ; R$-space of type $\left(B_{3},\left\{\alpha_{3}\right\}\right), W(Y)$ is identified with $G / P_{1}$ of type $\left(B_{3},\left\{\alpha_{2}, \alpha_{3}\right\}\right)$, where $C_{Y}^{*}$ and $N_{Y}$ correspond to $\mathfrak{g}_{-3} \oplus \mathfrak{g}_{-2} \oplus \mathfrak{g}_{-1}$ and $\mathfrak{g}_{-2} \oplus \mathfrak{g}_{-1}$ respectively for $\mathfrak{m}_{1}=\mathfrak{g}_{-4} \oplus \mathfrak{g}_{-3} \oplus \mathfrak{g}_{-2} \oplus \mathfrak{g}_{-1}$. Here $J=G / P_{2}$ of type $\left(B_{3},\left\{\alpha_{2}\right\}\right)$ is the standard contact manifold of $B_{3}$ type. Moreover $R(Y)$ is identified with $\mathrm{G} / \mathrm{B}$ of type $\left(B_{3},\left\{\alpha_{1} \cdot \alpha_{2}, \alpha_{3}\right\}\right)$, where $D_{Y}^{1}$ and $D_{Y}^{2}$ correspond to $\mathfrak{g}_{-4} \oplus \mathfrak{g}_{-3} \oplus \mathfrak{g}_{-2} \oplus \mathfrak{g}_{-1}$ and $\mathfrak{g}_{-2} \oplus \mathfrak{g}_{-1}$ respectively for $\mathfrak{m}_{0}=\mathfrak{g}_{-5} \oplus \mathfrak{g}_{-4} \oplus \mathfrak{g}_{-3} \oplus \mathfrak{g}_{-2} \oplus \mathfrak{g}_{-1}$.

To obtain an explicit description of the model equation in this case, we calculate

$$
\begin{aligned}
\varpi & =d y_{1}-x_{2} d x_{3}+\lambda_{1}\left(d y_{2}-x_{3} d x_{1}\right)+\lambda_{2}\left(d y_{3}-x_{1} d x_{2}\right) \\
& =d\left(y_{1}+\lambda_{1} y_{2}+\lambda_{2} y_{3}\right)-y_{2} d \lambda_{1}-y_{3} d \lambda_{2}-x_{2} d x_{3}-\lambda_{1} x_{3} d x_{1}-\lambda_{2} x_{1} d x_{2} \\
& =d\left(y_{1}+\lambda_{1} y_{2}+\lambda_{2} y_{3}-\lambda_{1} x_{1} x_{3}\right)-\left(y_{2}-x_{1} x_{3}\right) d \lambda_{1}-y_{3} d \lambda_{2}-\lambda_{2} x_{1} d x_{2}-\left(x_{2}-\lambda_{1} x_{1}\right) d x_{3} \\
& =d\left(y_{1}+\lambda_{1} y_{2}+\lambda_{2} y_{3}-\lambda_{1} x_{1} x_{3}-\lambda_{2} x_{1} x_{2}\right)-\left(y_{2}-x_{1} x_{3}\right) d \lambda_{1}-\left(y_{3}-x_{1} x_{2}\right) d \lambda_{2} \\
& \quad-\left(x_{2}-\lambda_{1} x_{1}\right)\left(d x_{3}-\lambda_{2} d x_{1}\right)+\lambda_{1} \lambda_{2} x_{1} d x_{1} \\
=d\left(y_{1}+\lambda_{1} y_{2}+\lambda_{2} y_{3}-\lambda_{1} x_{1} x_{3}-\lambda_{2} x_{1} x_{2}\right)- & \left(y_{2}-x_{1} x_{3}\right) d \lambda_{1}-\left(y_{3}-\lambda_{1} x_{1}^{2}\right) d \lambda_{2} \\
& -\left(x_{2}-\lambda_{1} x_{1}\right) d\left(x_{3}-\lambda_{2} x_{1}\right)+\frac{1}{2} \lambda_{1} \lambda_{2} d x_{1}^{2} \\
=d Z-P_{1} d X_{1}-P_{2} d X_{2}-P_{3} d X_{3} &
\end{aligned}
$$

Thus, putting

$$
\left\{\begin{array}{l}
Z=y_{1}+\lambda_{1} y_{2}+\lambda_{2} y_{3}-\lambda_{1} x_{1} x_{3}-\lambda_{2} x_{1} x_{2}+\frac{1}{2} \lambda_{1} \lambda_{2} x_{1}^{2}, X_{1}=\lambda_{1}, X_{2}=\lambda_{2}, X_{3}=x_{3}-\lambda_{2} x_{1}, \\
P_{1}=y_{2}-x_{1} x_{3}+\frac{1}{2} \lambda_{2} x_{1}^{2}, P_{2}=y_{3}-\frac{1}{2} \lambda_{1} x_{1}^{2}, P_{3}=x_{2}-\lambda_{1} x_{1},
\end{array}\right.
$$

we obtain a canonical coordinate $\left(X_{1}, X_{2}, X_{3}, Z, P_{1}, P_{2}, P_{3}\right)$ of $J=W(Y) / \mathrm{Ch}\left(C^{*}\right)$.

Conversely we calculate

$$
\left\{\begin{array}{l}
x_{1}=a, \lambda_{1}=X_{1}, \lambda_{2}=X_{2}, x_{3}=X_{3}+a X_{2}, x_{2}=P_{3}+a X_{1}, \\
y_{3}=P_{2}+\frac{1}{2} a^{2} X_{1}, y_{2}=P_{1}+a X_{3}+\frac{1}{2} a^{2} X_{2}
\end{array}\right.
$$

Hence we have

$$
\left\{\begin{array}{l}
\bar{\theta}_{2}=d y_{2}-x_{3} d x_{1}=d P_{1}+a d X_{3}+\frac{1}{2} a^{2} d X_{2} \\
\bar{\theta}_{3}=d y_{3}-x_{1} d x_{2}=d P_{2}-\frac{1}{2} a^{2} d X_{1}-a d P_{3}
\end{array}\right.
$$

Substituting $d P_{i}=P_{i 1} d P_{1}+P_{i 2} d P_{2}+P_{i 3} d P_{3}$ for $i=1,2,3$ into (8.2), we obtain the following description of the model equation of type $\left(B_{3},\left\{\alpha_{3}\right\}\right)$;

$$
P_{11}=0, P_{12}=-\frac{1}{2} P_{13}^{2}, P_{22}=P_{13}^{2} \cdot P_{33}, P_{23}=-P_{13} \cdot P_{33} .
$$

More generally, our second example is of type $\left(B D_{\ell},\left\{\alpha_{3}\right\}\right)$ for $\ell \geqq 4$. Explicitly, put

$$
S=\left(\begin{array}{ccc}
0 & 0 & K \\
0 & E_{p} & 0 \\
K & 0 & 0
\end{array}\right), \quad K=\left(\begin{array}{ccc}
0 & 0 & 1 \\
0 & 1 & 0 \\
1 & 0 & 0
\end{array}\right)
$$

where $K=K_{3}$ is the anti-diagonal unit $3 \times 3$ matrix and $E_{p}$ is the unit $p \times p$ matrix. On $\hat{U}=\mathbb{R}^{p+6}$, we give an inner product $($,$) by (\boldsymbol{x}, \boldsymbol{y})={ }^{t} \boldsymbol{x} S \boldsymbol{y}$ for $\boldsymbol{x}, \boldsymbol{y} \in \mathbb{R}^{p+6}$. Then the signature 
of $(\hat{U},()$,$) is (p+3,3)$. Moreover, on $U=\mathbb{R}^{p}$, we have an inner product $($,$) by (\mathbf{a}, \mathbf{b})={ }^{t} \mathbf{a b}$ for $\mathbf{a}, \mathbf{b} \in \mathbb{R}^{p}$.

We put

$$
\mathfrak{g}=\mathfrak{o}(\hat{U})=\mathfrak{o}(p+3,3)=\left\{X \in \mathfrak{g l}(p+6, \mathbb{R}) \mid{ }^{t} X S+S X=0\right\} .
$$

We will introduce the gradation of $\mathfrak{g}=\mathfrak{o}(\hat{U})$ by subdividing $X \in \mathfrak{g}$ as follows:

$$
\begin{gathered}
3 \\
3 \\
3
\end{gathered}\left(\begin{array}{ccc}
A & -\hat{F} & D \\
B & G & F \\
C & -\hat{B} & -A^{\prime}
\end{array}\right),
$$

where $C=-C^{\prime}, D=-D^{\prime}, G \in \mathfrak{o}(p) \hat{B}=K^{t} B$ and $\hat{F}=K^{t} F$. Here we write $Y^{\prime}=K^{t} Y K \in$ $M(3,3)$ for $Y \in M(3,3)$. $Y^{\prime}$ is the "transposed" matrix of $Y$ with respect to the anti-diagonal line. Then the Lie algebra $\mathfrak{g}$ has the gradation

$$
\mathfrak{g}=\mathfrak{g}_{-2}+\mathfrak{g}_{-1}+\mathfrak{g}_{0}+\mathfrak{g}_{1}+\mathfrak{g}_{2}
$$

where

$$
\mathfrak{g}_{-2}=\langle C\rangle, \mathfrak{g}_{-1}=\langle B\rangle, \mathfrak{g}_{0}=\langle A\rangle \oplus\langle G\rangle, \mathfrak{g}_{1}=\langle F\rangle, \mathfrak{g}_{2}=\langle D\rangle .
$$

and $\operatorname{dim} \mathfrak{g}_{-2}=\operatorname{dim} \mathfrak{g}_{2}=3, \operatorname{dim} \mathfrak{g}_{-1}=\operatorname{dim} \mathfrak{g}_{1}=3 p$. Precisely, this gradation is of type $\left(D_{4},\left\{\alpha_{3}, \alpha_{4}\right\}\right)$ when $p=2$, of type $\left(B_{\ell},\left\{\alpha_{3}\right\}\right)$ when $p=2 \ell-5 \geqq 1$ and of type $\left(D_{\ell},\left\{\alpha_{3}\right\}\right)$ when $p=2 \ell-6 \geqq 4$. The structure of $\mathfrak{m}=\mathfrak{g}_{-2} \oplus \mathfrak{g}_{-1}$ can be described as follows (see $\S 4$ [12]); Let $V$ be a vector space of dimension 3 and $U$ be a vector space with the inner product $($,$) of$ dimension $p$. Then $\mathfrak{m}$ is isomorphic to $\mathfrak{m}^{1}(U, V)$, where

$$
\mathfrak{m}^{1}(U, V)=\mathfrak{g}_{-2} \oplus \mathfrak{g}_{-1}, \quad \mathfrak{g}_{-2}=\wedge^{2} V, \mathfrak{g}_{-1}=U \otimes V .
$$

The bracket product is defined by

$$
\left[u_{1} \otimes v_{1}, u_{2} \otimes v_{2}\right]=\left(u_{1}, u_{2}\right) v_{1} \wedge v_{2} \quad \text { for } \quad u_{1}, u_{2} \in U, v_{1}, v_{2} \in V .
$$

Moreover the standard differential system $\left(M(\mathfrak{m}), D_{\mathfrak{m}}\right)$ of type $\mathfrak{m}$ in this case is given as follows; $M(\mathfrak{m})=\mathbb{R}^{3 p+3}$ is endowed with a coordinate $\left(x_{1}^{\alpha}, x_{2}^{\alpha}, x_{3}^{\alpha}, y_{1}, y_{2}, y_{3}\right)(1 \leqq \alpha \leqq p)$ such that $D_{\mathfrak{m}}$ is given by

$$
D_{\mathfrak{m}}=\left\{\bar{\theta}_{1}=\bar{\theta}_{2}=\bar{\theta}_{3}=0\right\}
$$

where

$$
\bar{\theta}_{1}=d y_{1}-\sum_{\alpha=1}^{p} x_{2}^{\alpha} d x_{3}^{\alpha}, \quad \bar{\theta}_{2}=d y_{2}-\sum_{\alpha=1}^{p} x_{3}^{\alpha} d x_{1}^{\alpha} \quad \text { and } \quad \bar{\theta}_{3}=d y_{3}-\sum_{\alpha=1}^{p} x_{1}^{\alpha} d x_{2}^{\alpha} .
$$

Thus the symbol algebra $\mathfrak{m}=\mathfrak{g}_{-2} \oplus \mathfrak{g}_{-1} \cong \mathfrak{m}^{1}(U, V)$ is described by

$$
\left\{\begin{aligned}
d \theta_{1} & \equiv \sum_{\alpha=1}^{p} \omega_{3}^{\alpha} \wedge \omega_{2}^{\alpha} \\
d \theta_{2} & \equiv \sum_{\alpha=1}^{p} \omega_{1}^{\alpha} \wedge \omega_{3}^{\alpha} \quad\left(\bmod \quad \theta_{1}, \theta_{2}, \theta_{3}\right) \\
d \theta_{3} & \equiv \sum_{\alpha=1}^{p} \omega_{2}^{\alpha} \wedge \omega_{1}^{\alpha}
\end{aligned}\right.
$$


In fact, taking the dual basis $\left\{\frac{\partial}{\partial y_{1}}, \frac{\partial}{\partial y_{2}}, \frac{\partial}{\partial y_{3}}, X_{1}^{\alpha}, X_{2}^{\alpha}, X_{3}^{\alpha}(1 \leqq \alpha \leqq p)\right\}$ of the coframe $\left\{\bar{\theta}_{1}, \bar{\theta}_{2}, \bar{\theta}_{3}\right.$, $\left.d x_{1}^{\alpha}, d x_{2}^{\alpha}, d x_{3}^{\alpha}(1 \leqq \alpha \leqq p)\right\}$ on $M(\mathfrak{m})$, we have

$$
X_{1}^{\alpha}=\frac{\partial}{\partial x_{1}^{\alpha}}+x_{3}^{\alpha} \frac{\partial}{\partial y_{2}}, \quad X_{2}^{\alpha}=\frac{\partial}{\partial x_{2}^{\alpha}}+x_{1}^{\alpha} \frac{\partial}{\partial y_{3}}, \quad \text { and } \quad X_{3}^{\alpha}=\frac{\partial}{\partial x_{3}^{\alpha}}+x_{2}^{\alpha} \frac{\partial}{\partial y_{1}} .
$$

Thus $\left\{X_{1}^{\alpha}, X_{2}^{\alpha}, X_{3}^{\alpha}(1 \leqq \alpha \leqq p)\right\}$ constitutes a free basis of the sections $\Gamma\left(D_{\mathfrak{m}}\right)$ of $D_{\mathfrak{m}}$, and we obtain

$$
\left[X_{1}^{\alpha}, X_{2}^{\beta}\right]=\delta_{\beta}^{\alpha} \frac{\partial}{\partial y_{3}},\left[X_{2}^{\alpha}, X_{3}^{\beta}\right]=\delta_{\beta}^{\alpha} \frac{\partial}{\partial y_{1}},\left[X_{3}^{\alpha}, X_{1}^{\beta}\right]=\delta_{\beta}^{\alpha} \frac{\partial}{\partial y_{2}},\left[X_{i}^{\alpha}, X_{i}^{\beta}\right]=0 \quad(i=1,2,3),
$$

for $1 \leqq \alpha, \beta \leqq p$. Hence $\mathfrak{m}$ is isomorphic to $\mathfrak{m}^{1}(U, V)$.

Let $\left(Y, D_{N}\right)$ be a regular differential system of type $\mathfrak{m}$ such that $D_{N}$ is locally defined by

$$
D_{N}=\left\{\theta_{1}=\theta_{2}=\theta_{3}=0\right\} .
$$

Here $\left\{\theta_{1}, \theta_{2}, \theta_{3}, \omega_{1}^{\alpha}, \omega_{2}^{\alpha}, \omega_{3}^{\alpha}(1 \leqq \alpha \leqq p)\right\}$ forms a coframe on $Y$ satisfying (8.3). Then, putting $\varpi=\theta_{1}+\lambda_{1} \theta_{2}+\lambda_{2} \theta_{3}$, we calculate

$$
\begin{aligned}
d \varpi & \equiv \sum_{\alpha=1}^{p} \omega_{3}^{\alpha} \wedge \omega_{2}^{\alpha}+\lambda_{1} \sum_{\alpha=1}^{p} \omega_{1}^{\alpha} \wedge \omega_{3}^{\alpha}+\lambda_{2} \sum_{\alpha=1}^{p} \omega_{2}^{\alpha} \wedge \omega_{1}^{\alpha}+\left(d \lambda_{1}+\gamma_{1}\right) \wedge \theta_{2}+\left(d \lambda_{2}+\gamma_{2}\right) \wedge \theta_{3} \\
& \equiv \sum_{\alpha=1}^{p}\left(\omega_{3}^{\alpha}-\lambda_{2} \omega_{1}^{\alpha}\right) \wedge\left(\omega_{2}^{\alpha}-\lambda_{1} \omega_{1}^{\alpha}\right)+\left(d \lambda_{1}+\gamma_{1}\right) \wedge \theta_{2}+\left(d \lambda_{2}+\gamma_{2}\right) \wedge \theta_{3} \\
& \equiv \sum_{\alpha=1}^{p} \tilde{\omega}_{3}^{\alpha} \wedge \tilde{\omega}_{2}^{\alpha}+\left(d \lambda_{1}+\gamma_{1}\right) \wedge \theta_{2}+\left(d \lambda_{2}+\gamma_{2}\right) \wedge \theta_{3} \quad(\bmod \quad \varpi)
\end{aligned}
$$

for some 1 -forms $\gamma_{1}, \gamma_{2}$ on $Y$, where we put $\tilde{\omega}_{2}^{\alpha}=\omega_{2}^{\alpha}-\lambda_{1} \omega_{1}^{\alpha}$ and $\tilde{\omega}_{3}^{\alpha}=\omega_{3}^{\alpha}-\lambda_{2} \omega_{1}^{\alpha}$ for $\alpha=1, \ldots, p$. Thus, by symmetry in the indices $1,2,3$ in (8.3), we see

$W(Y)=\hat{W}(Y)=\bigcup_{y \in Y} \tilde{W}_{y}, \quad \tilde{W}_{y}=\left\{w \in \operatorname{Gr}\left(T_{y}(Y), 3 p+2\right) \mid w \supset D_{N}(y)\right\} \cong \mathbb{P}\left(T_{y}(Y) / D_{N}(y)\right)=\mathbb{P}^{2}$ and we have on $W(Y)$,

$$
C_{Y}^{*}=\{\varpi=o\}, N_{Y}=\left\{\varpi=\theta_{2}=\theta_{3}=o\right\}, N_{Y}^{\perp}=\left\{\varpi=\theta_{2}=\theta_{3}=\tilde{\omega}_{2}^{\alpha}=\tilde{\omega}_{3}^{\alpha}=o(1 \leqq \alpha \leqq p)\right\} .
$$

Here $r=2, t=p, n=r+t=p+2$ and $\operatorname{dim} W(Y)=3 p+5$. Moreover we have

$$
\operatorname{Ch}\left(C_{Y}^{*}\right)=\left\{\varpi=\theta_{2}=\theta_{3}=d \lambda_{1}+\gamma_{1}=d \lambda_{2}+\gamma_{2}=\tilde{\omega}_{2}^{\alpha}=\tilde{\omega}_{3}^{\alpha}=o(1 \leqq \alpha \leqq p)\right\} .
$$

Now we consider the Lagrange Grassmann bundle $R(Y)=R(W(Y))$ over $\left(W(Y) ; C_{Y}^{*}, N_{Y}\right)$ :

$$
R(Y)=\bigcup_{w \in W(Y)} R_{w}, \quad R_{w}=\left\{\hat{v} \subset N_{Y}(w)|d \varpi|_{\hat{v}}=0, \quad \hat{v} \text { is maximal }\right\} .
$$

From $N_{Y}^{\perp}(w) \subset \hat{v} \subset N_{Y}(w)$ and $\left.d \varpi\right|_{\hat{v}}=0$, if $\left.\tilde{\omega}_{2}^{1} \wedge \cdots \wedge \tilde{\omega}_{2}^{p}\right|_{\hat{v}} \neq 0$, it follows that

$$
\hat{v}=\left\{X \in N_{Y}(w) \mid \varpi_{\alpha}(X)=0 \quad(1 \leqq \alpha \leqq p)\right\},
$$

where $\varpi_{\alpha}=\tilde{\omega}_{3}^{\alpha}-\sum_{\beta=1}^{p} p_{\alpha \beta}^{*} \tilde{\omega}_{2}^{\beta}$ for $1 \leqq \alpha \leqq p$ and $p_{\alpha \beta}^{*}=p_{\beta \alpha}^{*}(1 \leqq \alpha, \beta \leqq p)$. For these $\hat{v} \in R_{w}$, we claim

$$
A(\hat{v})=\{0\} \quad \text { for } \quad \hat{v}=\left\{\varpi=\theta_{2}=\theta_{3}=\varpi_{\alpha}=0(1 \leqq \alpha \leqq p)\right\} .
$$

In fact, first we have

$$
d \theta_{2} \equiv \sum_{\alpha=1}^{p} \omega_{1}^{\alpha} \wedge \tilde{\omega}_{3}^{\alpha}, \quad d \theta_{3} \equiv \sum_{\alpha=1}^{p} \tilde{\omega}_{2}^{\alpha} \wedge \omega_{1}^{\alpha} \quad\left(\bmod \quad \varpi, \theta_{2}, \theta_{3}\right) .
$$


Thus we get

$$
d \varpi \equiv 0, \quad d \theta_{2} \equiv \sum_{\alpha, \beta=1}^{p} p_{\alpha \beta}^{*} \omega_{1}^{\alpha} \wedge \tilde{\omega}_{2}^{\beta}, \quad d \theta_{3} \equiv \sum_{\alpha=1}^{p} \tilde{\omega}_{2}^{\alpha} \wedge \omega_{1}^{\alpha} \quad\left(\bmod \quad(\hat{v})^{\perp}\right)
$$

Hence, for $X \in S\left(N_{Y}\right)(w)=\mathrm{Ch}\left(C_{Y}^{*}\right)(w)$, we have

$$
X\rfloor d \theta_{3} \equiv-\sum_{\alpha=1}^{p} \omega_{1}^{\alpha}(X) \tilde{\omega}_{2}^{\alpha} \quad\left(\bmod \quad(\hat{v})^{\perp}\right)
$$

Then, from Lemma 4.2 (2), we obtain

$$
\begin{aligned}
A(\hat{v}) & =\left\{X \in \operatorname{Ch}\left(C_{Y}^{*}\right)(w) \mid \omega_{1}^{\alpha}(X)=0 \quad(1 \leqq \alpha \leqq p)\right\} \\
& =\operatorname{Ch}\left(C_{Y}^{*}\right)(w) \cap \operatorname{Ch}\left(N_{Y}\right)(w)=\{0\} .
\end{aligned}
$$

Hence $\left(R(Y) ; D_{Y}^{1}, D_{Y}^{2}\right)$ is a $P D$ manifold of second order on an open subset of $R(Y)$, when $\left(Y, D_{N}\right)$ is a regular differential system of type $\mathfrak{m}$, where $\mathfrak{m}$ is the negative part of the simple graded Lie algebra of type $\left(B D_{\ell},\left\{\alpha_{3}\right\}\right)$.

To obtain an explicit description of the model equation in this case, we calculate

$$
\begin{aligned}
\varpi & =d y_{1}-\sum_{\alpha=1}^{p} x_{2}^{\alpha} d x_{3}^{\alpha}+\lambda_{1}\left(d y_{2}-\sum_{\alpha=1}^{p} x_{3}^{\alpha} d x_{1}^{\alpha}\right)+\lambda_{2}\left(d y_{3}-\sum_{\alpha=1}^{p} x_{1}^{\alpha} d x_{2}^{\alpha}\right) \\
& =d\left(y_{1}+\lambda_{1} y_{2}+\lambda_{2} y_{3}\right)-y_{2} d \lambda_{1}-y_{3} d \lambda_{2}-\sum_{\alpha=1}^{p} x_{2}^{\alpha} d x_{3}^{\alpha}-\lambda_{1} \sum_{\alpha=1}^{p} x_{3}^{\alpha} d x_{1}^{\alpha}-\lambda_{2} \sum_{\alpha=1}^{p} x_{1}^{\alpha} d x_{2}^{\alpha} \\
& =d\left(y_{1}+\lambda_{1} y_{2}+\lambda_{2} y_{3}-\lambda_{1} \sum_{\alpha=1}^{p} x_{1}^{\alpha} x_{3}^{\alpha}\right)-\left(y_{2}-\sum_{\alpha=1}^{p} x_{1}^{\alpha} x_{3}^{\alpha}\right) d \lambda_{1}-y_{3} d \lambda_{2}-\lambda_{2} \sum_{\alpha=1}^{p} x_{1}^{\alpha} d x_{2}^{\alpha} \\
& =d\left(y_{1}+\lambda_{1} y_{2}+\lambda_{2} y_{3}-\lambda_{1} \sum_{\alpha=1}^{p} x_{1}^{\alpha} x_{3}^{\alpha}-\lambda_{2} \sum_{\alpha=1}^{p} x_{1}^{\alpha} x_{2}^{\alpha}\right)-\left(y_{2}-\sum_{\alpha=1}^{p} x_{1}^{\alpha} x_{3}^{\alpha}\right) d \lambda_{1} \\
& -\left(y_{3}-\sum_{\alpha=1}^{p} x_{1}^{\alpha} x_{2}^{\alpha}\right) d \lambda_{2}-\sum_{\alpha=1}^{p}\left(x_{2}^{\alpha}-\lambda_{1} x_{1}^{\alpha}\right)\left(d x_{3}^{\alpha}-\lambda_{2} d x_{1}^{\alpha}\right)+\lambda_{1} \lambda_{2} \sum_{\alpha=1}^{p} x_{1}^{\alpha} d x_{1}^{\alpha} \\
& =d\left(y_{1}+\lambda_{1} y_{2}+\lambda_{2} y_{3}-\lambda_{1} \sum_{\alpha=1}^{p} x_{1}^{\alpha} x_{3}^{\alpha}-\lambda_{2} \sum_{\alpha=1}^{p} x_{1}^{\alpha} x_{2}^{\alpha}\right)-\left(y_{2}-\sum_{\alpha=1}^{p} x_{1}^{\alpha} x_{3}^{\alpha}\right) d \lambda_{1} \\
& -\left(y_{3}-\lambda_{1} \sum_{\alpha=1}^{p}\left(x_{1}^{\alpha}\right)^{2}\right) d \lambda_{2}-\sum_{\alpha=1}^{p}\left(x_{2}^{\alpha}-\lambda_{1} x_{1}^{\alpha}\right) d\left(x_{3}^{\alpha}-\lambda_{2} x_{1}^{\alpha}\right)+\frac{1}{2} \lambda_{1} \lambda_{2} \sum_{\alpha=1}^{p} d\left(x_{1}^{\alpha}\right)^{2} \\
& =d Z-P_{1} d X_{1}-P_{2} d X_{2}-\sum_{\alpha=1}^{p} P_{\alpha+2} d X_{\alpha+2}
\end{aligned}
$$


Thus, putting

$$
\left\{\begin{array}{l}
Z=y_{1}+\lambda_{1} y_{2}+\lambda_{2} y_{3}-\lambda_{1} \sum_{\alpha=1}^{p} x_{1}^{\alpha} x_{3}^{\alpha}-\lambda_{2} \sum_{\alpha=1}^{p} x_{1}^{\alpha} x_{2}^{\alpha}+\frac{1}{2} \lambda_{1} \lambda_{2} \sum_{\alpha=1}^{p}\left(x_{1}^{\alpha}\right)^{2}, \\
X_{1}=\lambda_{1}, X_{2}=\lambda_{2}, X_{\alpha+2}=x_{3}^{\alpha}-\lambda_{2} x_{1}^{\alpha}, \\
P_{1}=y_{2}-\sum_{\alpha=1}^{p} x_{1}^{\alpha} x_{3}^{\alpha}+\frac{1}{2} \lambda_{2} \sum_{\alpha=1}^{p}\left(x_{1}^{\alpha}\right)^{2}, P_{2}=y_{3}-\frac{1}{2} \lambda_{1} \sum_{\alpha=1}^{p}\left(x_{1}^{\alpha}\right)^{2}, P_{\alpha+2}=x_{2}^{\alpha}-\lambda_{1} x_{1}^{\alpha},
\end{array}\right.
$$

we obtain a canonical coordinate $\left(X_{1}, \ldots, X_{p+2}, Z, P_{1}, \ldots, P_{p+2}\right)$ of $J=W(Y) / \mathrm{Ch}\left(C_{Y}^{*}\right)$.

Conversely we calculate

$$
\left\{\begin{array}{l}
x_{1}^{\alpha}=a_{\alpha}, \lambda_{1}=X_{1}, \lambda_{2}=X_{2}, x_{3}^{\alpha}=X_{\alpha+2}+a_{\alpha} X_{2}, x_{2}^{\alpha}=P_{\alpha+2}+a_{\alpha} X_{1}, \\
y_{3}=P_{2}+\frac{1}{2} \sum_{\alpha=1}^{p}\left(a_{\alpha}\right)^{2} X_{1}, y_{2}=P_{1}+\sum_{\alpha=1}^{p} a_{\alpha} X_{\alpha+2}+\frac{1}{2} \sum_{\alpha=1}^{p}\left(a_{\alpha}\right)^{2} X_{2}
\end{array}\right.
$$

Hence we have

$$
\left\{\begin{array}{l}
\bar{\theta}_{2}=d y_{2}-\sum_{\alpha=1}^{p} x_{3}^{\alpha} d x_{1}^{\alpha}=d P_{1}+\sum_{\alpha=1}^{p} a_{\alpha} d X_{\alpha+2}+\frac{1}{2} \sum_{\alpha=1}^{p}\left(a_{\alpha}\right)^{2} d X_{2} \\
\bar{\theta}_{3}=d y_{3}-\sum_{\alpha=1}^{p} x_{1}^{\alpha} d x_{2}^{\alpha}=d P_{2}-\frac{1}{2} \sum_{\alpha=1}^{p}\left(a_{\alpha}\right)^{2} d X_{1}-\sum_{\alpha=1}^{p} a_{\alpha} d P_{\alpha+2}
\end{array}\right.
$$

Substituting $d P_{i}=\sum_{j=1}^{p+2} P_{i j} d X_{j}$ for $i=1, \ldots, p+2$ into (8.4), we obtain the following description of the model equation of type $\left(B D_{\ell},\left\{\alpha_{3}\right\}\right)$;

$$
P_{11}=0, \quad P_{12}=-\frac{1}{2} \sum_{\alpha=1}^{p}\left(P_{1 \alpha+2}\right)^{2}, \quad P_{2 i}=-\sum_{\alpha=1}^{p} P_{1 \alpha+2} \cdot P_{i \alpha+2} \quad(i=2, \ldots, p+2) .
$$

8.3. General Case. In this subsection, we will first consider the third reduction step in Theorem 5.2 in general and will discuss the reconstruction procedure of this third step. As in Theorem 5.2, let $\left(R ; D^{1}, D^{2}\right)$ be a $P D$ manifold of second order, which is regular of type $\mathfrak{s}$. Assume that there exists a $G_{0}(\mathfrak{s})$-invariant subspace $E$ of $V$ of dimension $r$ satisfying $\mathfrak{f}^{\perp} \subset E \otimes_{S} V$ and $\operatorname{dim} \hat{E}=s>0$, where $\hat{E}=\left\{v \in E \mid v \odot E \subset \mathfrak{f}^{\perp}\right\}$. Moreover assume the following two conditions:

(i) $C(E)$ is completely integrable (when $r=n-1$, assume further $\operatorname{rank} \mathrm{Ch}\left(D^{2}\right)<s$ ).

(ii) $C(E) \subset \mathrm{Ch}\left(\tilde{N}^{*}\right)$.

Let $\left(W ; C^{*}, N\right)$ be the associated $I G$ manifolds of corank $\mathrm{r}$ of $\left(R ; D^{1}, D^{2}\right)$. Assume that $W$ is regular with respect to $\mathrm{Ch}(N)$ and let $\left(Y ; D_{N}^{*}, D_{N}\right)$ be the leaf space, where $\beta: W \rightarrow$ $Y=W / \mathrm{Ch}(N)$ is the projection, $\beta^{-1}\left(D_{N}^{*}\right)=N^{*}$ and $\beta^{-1}\left(D_{N}\right)=N$. Then, as in $\S 5.3$, we have $\mathrm{Ch}\left(D_{N}^{*}\right) \subset D_{N}$ and $\mathrm{Ch}\left(D_{N}\right)=\{0\}$. Now we further assume that $\mathrm{Ch}\left(D_{N}^{*}\right)$ is a non-trivial subbundle of $D_{N}$ such that $Y$ is regular with respect to $\mathrm{Ch}\left(D_{N}^{*}\right)$, i.e., the space $Z=Y / \mathrm{Ch}\left(D_{N}^{*}\right)$ of leaves of this foliation is a manifold and that each fibre of the projection $\gamma: Y \rightarrow Z$ is connected and $\gamma$ is a submersion. Then there exists a differential system $F$ on $Z$ of codimension $s+1$ such that $\gamma_{*}^{-1}(F)=D_{N}^{*}$ and $\mathrm{Ch}(F)$ is trivial. We consider here a Grassmann bundle $\tilde{Y}(Z)$ over $Z$ consisting of subspaces of codimension $r-s$ in each fibre $F(z)$ of $(Z, F)$.

$$
\tilde{Y}(Z)=\bigcup_{z \in Z} \tilde{Y}_{z}, \quad \tilde{Y}_{z}=\operatorname{Gr}\left(F(z), t_{0}\right)
$$


where $t_{0}+r-s=\operatorname{rank} F$. Then we have two differential systems $F_{N}^{*}, F_{N}$ on $\tilde{Y}(Z)$ given by

$$
F_{N}^{*}(\tilde{y})=\xi_{*}^{-1}(F(z)) \supset F_{N}(\tilde{y})=\xi_{*}^{-1}(\tilde{y})
$$

for each $\tilde{y} \in \tilde{Y}(Z)$ and $z=\xi(\tilde{y})$, where $\xi: \tilde{Y}(Z) \rightarrow Z$ is the projection. Hence $F_{N}^{*}$ and $F_{N}$ are differential systems on $\tilde{Y}(Z)$ of codimension $s+1$ and $r+1$ respectively. In this situation, we have a map $\kappa_{3}$ of $Y$ into $\tilde{Y}(Z)$ given by

$$
\kappa_{3}(y)=\gamma_{*}\left(D_{N}(y)\right) \subset F(z)=\gamma_{*}\left(D_{N}^{*}(y)\right),
$$

for each $y \in Y$ and $z=\gamma(y)$. By the Realization Lemma for $\left(Y, D_{N}, \gamma, Z\right), \kappa_{3}$ is a map of constant rank such that

$$
\operatorname{Ker}\left(\kappa_{3}\right)_{*}=\operatorname{Ch}\left(D_{N}\right) \cap \operatorname{Ker} \gamma_{*}=\{0\} .
$$

Thus $\kappa_{3}$ is an immersion. Moreover we have

$$
\left(\kappa_{3}\right)_{*}^{-1}\left(F_{N}^{*}\right)=D_{N}^{*} \quad \text { and } \quad\left(\kappa_{3}\right)_{*}^{-1}\left(F_{N}\right)=D_{N} .
$$

Namely $\kappa_{3}$ is an immersion of $\left(Y ; D_{N}^{*}, D_{N}\right)$ into $\left(\tilde{Y}(Z) ; F_{N}^{*}, F_{N}\right)$. Hence $\left(Y ; D_{N}^{*}, D_{N}\right)$ can be constructed from $(Z, F)$, at least locally, as a submanifold of $\left(\tilde{Y}(Z) ; F_{N}^{*}, F_{N}\right)$.

Furthermore, starting from $\gamma: Y \rightarrow Z$, we have the following general picture: Starting from $(Z, F)$, by the construction in $\S 7.1$, we have an $I G$ manifold $\left(W(Z) ; C_{Z}^{*}, N_{Z}\right)$ of corank $s$ and the Lagrange Grassmann bundle $R(Z)=R(W(Z))$ over $\left(W(Z) ; C_{Z}^{*}, N_{Z}\right)$ as follows. Put

$$
\hat{W}(Z)=\bigcup_{z \in Z} \hat{W}_{z}, \quad \hat{W}_{z}=\left\{w \in \operatorname{Gr}\left(T_{z}(Z), \hat{m}-1\right) \mid w \supset F(z)\right\} \cong \mathbb{P}\left(T_{z}(Z) / F(z)\right)=\mathbb{P}^{s},
$$

where $\hat{m}=\operatorname{dim} Z, C_{Z}^{*}$ is the canonical system obtained by the Grassmannian construction and $N_{Z}$ is the lift of $F$. Moreover we put

$$
W(Z)=\left\{w \in \hat{W}(Z) \mid \operatorname{corank} \operatorname{Ch}\left(C_{Z}^{*}\right)(w)=2 n+1\right\},
$$

where $n=s+t$ and $t$ is the Engel half-rank of $F$. Here we note that $t$ is also the Engel half-rank of $D_{N}^{*}$. Then, by Proposition $7.1,\left(W(Z) ; C_{Z}^{*}, N_{Z}\right)$ is an $I G$ manifold of corank $s$. Let $\left(R(Z) ; D_{Z}^{1}, D_{Z}^{2}\right)$ be the Lagrange Grassmann bundle over $\left(W(Z) ; C_{Z}^{*}, N_{Z}\right)$, i.e.,

$$
R(Z)=\bigcup_{w \in W(Z)} R_{w}, \quad R_{W}=\left\{\hat{v} \subset N_{Z}(w)\left|\gamma_{w}\right|_{\hat{v}}=0, \quad \hat{v} \text { is maximal }\right\}
$$

where $D_{Z}^{2}$ is the canonical system on $R(Z)$ and $D_{Z}^{1}$ and $\hat{N}_{Z}$ are the lifts of $C_{Z}^{*}$ and $N_{Z}$ respectively. Assume that $W(Z)$ is regular with respect to $\mathrm{Ch}\left(C_{Z}^{*}\right)$. Then we have a contact manifold $(J, C)$ such that $J=W(Z) / \mathrm{Ch}\left(C_{Z}^{*}\right)$ and $C_{Z}^{*}=q_{*}^{-1}(C)$, where $q: W(Z) \rightarrow J$ is the projection. Here we have a map $\hat{\zeta}: R(Z) \rightarrow L(J)$ given by $\hat{\zeta}(\hat{v})=q_{*}(\hat{v})$. $\hat{\zeta}$ is an immersion when $\left(R(Z) ; D_{Z}^{1}, D_{Z}^{2}\right)$ is a $P D$ manifold of second order.

On the other hand, starting from $\left(Y ; D_{N}^{*}, D_{N}\right)$, by the construction in $\S 7.2$, we have an $I G$ manifold $\left(W(Y) ; C_{Y}^{*}, N_{Y}\right)$ of corank $r$ and the Lagrange Grassmann bundle $R(Y)=R(W(Y))$ over $\left(W(Y) ; C_{Y}^{*} \cdot N_{Y}\right)$. In case $\left(Y ; D_{N}^{*}, D_{N}\right)$ is obtained from a $P D$ manifold $\left(R ; D^{1}, D^{2}\right)$ of second order of type $\mathfrak{s}$ as above, we have local isomorphisms $\kappa_{2}:\left(W ; C^{*}, N\right) \rightarrow\left(W(Y), C_{Y}^{*}, N_{Y}\right)$ and $\kappa_{1}:\left(R ; D^{1}, D^{2}\right) \rightarrow\left(R(W) ; D_{W}^{1}, D_{W}^{2}\right)$ given by $\kappa_{2}(w)=\beta_{*}\left(C^{*}(w)\right)$ and $\kappa_{1}(v)=\eta_{*}\left(D^{2}(v)\right)$ respectively (see $\S 5.1$ and $\S 5.3)$, so that we have a local isomorphism $\hat{\kappa}_{1}=\hat{\kappa}_{2} \cdot \kappa_{1}:\left(R ; D^{1}, D^{2}\right) \rightarrow$ $\left(R(Y) ; D_{Y}^{1}, D_{Y}^{2}\right)$, where the local isomorphism $\hat{\kappa}_{2}:\left(R(W) ; D_{W}^{1}, D_{W}^{2}\right) \rightarrow\left(R(Y) ; D_{Y}^{1}, D_{Y}^{2}\right)$ is 
induced by $\kappa_{2}$. In this situation, $\gamma: Y \rightarrow Z$ induces the following commutative diagram:

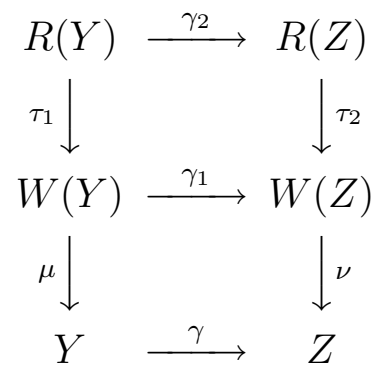

In fact, for $w \in W(Y), w$ is a hyperplane in $T_{y}(Y)$ containing $D_{N}^{*}(y)$, where $y=\mu(w)$. Then, since $D_{N}^{*}(y) \supset \operatorname{Ch}\left(D_{N}^{*}\right)(y)=\operatorname{Ker} \gamma_{*}(y)$ and $\gamma_{*}\left(D_{N}^{*}(y)\right)=F(z), \gamma_{*}(w)$ is a hyperplane in $T_{z}(Z)$ containing $F(z)$, where $z=\gamma(y)$. Hence $\gamma_{1}: W(Y) \rightarrow W(Z)$ is defined by $\gamma_{1}(w)=\gamma_{*}(w) \in W(Z)$ for $w \in W(Y)$. Actually $\gamma_{1}: \tilde{W}(Y) \rightarrow \hat{W}(Z)$ is a $\mathbb{P}^{s}$-bundle homomorphism. Passing to the tangent map, since $\mu_{*}^{-1}(w)=C_{Y}^{*}(w)$ and $\nu_{*}^{-1}\left(\gamma_{1}(w)\right)=C_{Z}^{*}\left(\gamma_{1}(w)\right)$, we have $\left(\gamma_{1}\right)_{*}\left(C_{Y}^{*}(w)\right)=C_{Z}^{*}\left(\gamma_{1}(w)\right)$ and $\left(\gamma_{1}\right)_{*}\left(\operatorname{Ch}\left(C_{Y}^{*}\right)(w)\right)=\operatorname{Ch}\left(C_{Z}^{*}\right)\left(\gamma_{1}(w)\right)$ for $w \in W(Y)$. Moreover, since $\mu_{*}^{-1}\left(D_{N}^{*}(y)\right)=N_{Y}^{*}(w)$ and $\nu_{*}^{-1}(F(\gamma(y)))=N_{Z}\left(\gamma_{1}(w)\right)$, we have $\left(\gamma_{1}\right)_{*}\left(N_{Y}^{*}(w)\right)=$ $N_{Z}\left(\gamma_{1}(w)\right)$. Hence $\gamma_{1}$ naturally induces the map $\gamma_{2}: R(Y) \rightarrow R(Z)$ by $\gamma_{2}(\hat{v})=\left(\gamma_{1}\right)_{*}(\hat{v})$ for $\hat{v} \in$ $R(Y)$. In fact, since $C_{Z}^{*}=q_{*}^{-1}(C)$ for the projection $q: W(Z) \rightarrow J$, we have $C_{Y}^{*}=\left(q \cdot \gamma_{1}\right)_{*}^{-1}(C)$ and $\operatorname{Ker}\left(q \cdot \gamma_{1}\right)_{*}=\operatorname{Ch}\left(C_{Y}^{*}\right)$, which implies that $J=W(Y) / \mathrm{Ch}\left(C_{Y}^{*}\right)$ at least locally. Thus we see that, for a subspace $\hat{v}$ of $T_{w}(W(Y)), \hat{v} \in R(Y)$ if and only if $\operatorname{Ch}\left(C_{Y}^{*}\right)(w) \subset \hat{v} \subset N_{Y}(w)$ and $\left(q \cdot \gamma_{1}\right)_{*}(\hat{v})$ is a legendrian subspace of $(J, C)$. Similarly, for a subspace $\tilde{v}$ of $T_{\hat{w}}(W(Z)), \tilde{v} \in R(Z)$ if and only if $\mathrm{Ch}\left(C_{Z}^{*}\right)(\hat{w}) \subset \tilde{v} \subset N_{Z}(\hat{w})$ and $q_{*}(\tilde{v})$ is a legendrian subspace of $(J, C)$. Hence, from $\left(\gamma_{1}\right)_{*}\left(N_{Y}(w)\right) \subset\left(\gamma_{1}\right)_{*}\left(N_{Y}^{*}(w)\right)=N_{Z}\left(\gamma_{1}(w)\right)$, we have $\gamma_{2}(\hat{v})=\left(\gamma_{1}\right)_{*}(\hat{v}) \in R(Z)$. Here we observe that both $\left(q \cdot \gamma_{1}\right)_{*}\left(N_{Y}(w)\right)$ and $q_{*}\left(N_{Z}\left(\gamma_{1}(w)\right)\right)$ are involutive subspaces of $C\left(q \cdot \gamma_{1}(w)\right)$ of codimension $r$ and $s$ respectively such that $\left(q \cdot \gamma_{1}\right)_{*}\left(N_{Y}(w)\right) \subset q_{*}\left(N_{Z}\left(\gamma_{1}(w)\right)\right)$.

Now we will give examples of constructions in $\$ 7.2$. Our starting point here is a regular differential system $(Z, D)$ of type $\mathfrak{m}_{3}$, where $\mathfrak{m}_{3}=\mathfrak{g}_{-3} \oplus \mathfrak{g}_{-2} \oplus \mathfrak{g}_{-1}$ is the negative part of the simple graded Lie algebra of type $\left(B D_{\ell},\left\{\alpha_{1}, \alpha_{3}\right\}\right)$, which is discussed in $\S 6.2$ [27] related with the First Reduction Theorem. We will show that, starting from $(Z, F)$, where $F=\partial D$ is the derived system of $D$, we can construct an involutive system $\left(R(Y) ; D_{Y}^{1}, D_{Y}^{2}\right)$ of second order of codimensin 2 in the above picture, by suitably constructing $\left(Y ; D_{N}^{*}, D_{N}\right)$ over $(Z, F)$.

For this purpose, let us first describe the structure of the symbol algebra $\mathfrak{m}_{3}$. Explicitly, as in $\S 8.1$, put

$$
S=\left(\begin{array}{ccccc}
0 & 0 & 0 & 0 & 1 \\
0 & 0 & 0 & K & 0 \\
0 & 0 & E_{p} & 0 & 0 \\
0 & K & 0 & 0 & 0 \\
1 & 0 & 0 & 0 & 0
\end{array}\right), \quad K=\left(\begin{array}{cc}
0 & 1 \\
1 & 0
\end{array}\right)
$$

where $K=K_{2}$ is the anti-diagonal unit $2 \times 2$ matrix and $E_{p}$ is the unit $p \times p$ matrix. We put

$$
\mathfrak{g}=\mathfrak{o}(p+3,3)=\left\{X \in \mathfrak{g l}(p+6, \mathbb{R}) \mid{ }^{t} X S+S X=0\right\} .
$$

We will introduce the gradation of $\mathfrak{g}$ by subdividing $X \in \mathfrak{g}$ as follows:

$$
\begin{aligned}
& 1 \\
& 2 \\
& p \\
& 2 \\
& 1
\end{aligned}\left(\begin{array}{ccccc}
1 & 2 & p & 2 & 1 \\
\eta_{1} & -\eta_{4}^{\prime} & -{ }^{t} \xi_{2} & -\eta_{3}^{\prime} & 0 \\
\xi_{1} & B_{1} & -\hat{B}_{2} & C_{2} & \eta_{3} \\
\eta_{2} & C_{1} & -\hat{B}_{1} & -A_{2} & \xi_{2} \\
0 & -\eta_{2}^{\prime} & -{ }^{t} \xi_{1} & -\eta_{1}^{\prime} & -a
\end{array}\right),
$$


where $a \in \mathbb{R}, \xi_{i} \in \mathbb{R}^{p}(i=1,2), \eta_{i} \in \mathbb{R}^{2}(i=1,2,3,4), \eta_{i}^{\prime}=\left(a_{2}, a_{1}\right)$ for $\eta_{i}={ }^{t}\left(a_{1}, a_{2}\right), C_{i}=-C_{i}^{\prime}$ $(i=1,2), G \in \mathfrak{o}(p)$ and $\hat{B}_{i}={ }^{t}\left({ }^{t} \mathfrak{b}_{2},{ }^{t} \mathfrak{b}_{1}\right)$ for $B_{i}=\left(\mathfrak{b}_{1}, \mathfrak{b}_{2}\right)(i=1,2)$, where $\mathfrak{b}_{1}, \mathfrak{b}_{2} \in \mathbb{R}^{p}$. Here we write $Y^{\prime}=K^{t} Y K \in M(2,2)$ for $Y \in M(2,2)$. $Y^{\prime}$ is the "transposed" matrix of $Y$ with respect to the anti-diagonal line. Thus $C_{i}=\left(\begin{array}{cc}c_{i} & 0 \\ 0 & -c_{i}\end{array}\right)$ for $c_{i} \in \mathbb{R}$. Then the Lie algebra $\mathfrak{g}$ has the gradation

$$
\mathfrak{g}=\mathfrak{g}_{-3} \oplus \mathfrak{g}_{-2} \oplus \mathfrak{g}_{-1} \oplus \mathfrak{g}_{0} \oplus \mathfrak{g}_{1} \oplus \mathfrak{g}_{2} \oplus \mathfrak{g}_{3}
$$

where

$$
\begin{aligned}
\mathfrak{g}_{-3}=\left\langle\eta_{2}\right\rangle, \mathfrak{g}_{-2} & =\left\langle\xi_{1}\right\rangle \oplus\left\langle C_{1}\right\rangle, \mathfrak{g}_{-1}=\left\langle\eta_{1}\right\rangle \oplus\left\langle B_{1}\right\rangle, \mathfrak{g}_{0}=\langle a\rangle \oplus\langle A\rangle \oplus\langle G\rangle, \\
\mathfrak{g}_{3} & =\left\langle\eta_{3}\right\rangle, \mathfrak{g}_{2}=\left\langle\xi_{2}\right\rangle \oplus\left\langle C_{2}\right\rangle, \mathfrak{g}_{1}=\left\langle\eta_{4}\right\rangle \oplus\left\langle B_{2}\right\rangle,
\end{aligned}
$$

and $\operatorname{dim} \mathfrak{g}_{-3}=\operatorname{dim} \mathfrak{g}_{3}=2, \operatorname{dim} \mathfrak{g}_{-2}=\operatorname{dim} \mathfrak{g}_{2}=p+1, \operatorname{dim} \mathfrak{g}_{-1}=\operatorname{dim} \mathfrak{g}_{1}=2(p+1)$. Precisely, this gradation is of type $\left(D_{4},\left\{\alpha_{1}, \alpha_{3}, \alpha_{4}\right\}\right)$ when $p=2$, of type $\left(B_{\ell},\left\{\alpha_{1}, \alpha_{3}\right\}\right)$ when $p=2 \ell-5 \geqq 1$ and of type $\left(D_{\ell},\left\{\alpha_{1}, \alpha_{3}\right\}\right)$ when $p=2 \ell-6 \geqq 4$. Compared with the gradation of $\left(B D_{\ell},\left\{\alpha_{1}, \alpha_{2}, \alpha_{3}\right\}\right)$ or $\left(D_{4},\left\{\alpha_{1}, \alpha_{2}, \alpha_{3}, \alpha_{4}\right\}\right)$;

$$
\mathfrak{g}=\hat{\mathfrak{g}}_{-5} \oplus \hat{\mathfrak{g}}_{-4} \oplus \hat{\mathfrak{g}}_{-3} \oplus \hat{\mathfrak{g}}_{-2} \oplus \hat{\mathfrak{g}}_{-1} \oplus \hat{\mathfrak{g}}_{0} \oplus \hat{\mathfrak{g}}_{1} \oplus \hat{\mathfrak{g}}_{2} \oplus \hat{\mathfrak{g}}_{3} \oplus \hat{\mathfrak{g}}_{4} \oplus \hat{\mathfrak{g}}_{5},
$$

which is obtained by further subdividing above matrix $X \in \mathfrak{g}$ by 2 nd and $(p+4)$-th intermediate lines (see $\S 6.2[27]$ ), we have

$$
\mathfrak{g}_{-3}=\hat{\mathfrak{g}}_{-5} \oplus \hat{\mathfrak{g}}_{-4}, \quad \mathfrak{g}_{-2}=\hat{\mathfrak{g}}_{-3} \quad \text { and } \quad \mathfrak{g}_{-1}=V_{2} \oplus V_{1} \subset \hat{\mathfrak{g}}_{-2} \oplus \hat{\mathfrak{g}}_{-1},
$$

where $V_{2}=\hat{\mathfrak{g}}_{-2}$ and $V_{1}=\hat{\mathfrak{g}}_{-1} \cap \mathfrak{g}_{-1}$. Explicitly we have

$$
\hat{\mathfrak{g}}_{-5}=\left\langle{ }^{t}\left(0, a_{2}\right)\right\rangle, \quad \hat{\mathfrak{g}}_{-4}=\left\langle{ }^{t}\left(a_{1}, 0\right)\right\rangle, \quad V_{2}=\left\langle{ }^{t}\left(0, a_{2}\right)\right\rangle \oplus\left\langle\left(\mathfrak{b}_{1}, 0\right)\right\rangle, \quad V_{1}=\left\langle{ }^{t}\left(a_{1}, 0\right)\right\rangle \oplus\left\langle\left(0, \mathfrak{b}_{2}\right)\right\rangle .
$$

Hence we get

$$
\left[V_{i}, V_{i}\right]=0(i=1,2), \quad\left[V_{1}, V_{2}\right] \subset \mathfrak{g}_{-2}, \quad\left[\mathfrak{g}_{-2}, V_{2}\right]=\hat{\mathfrak{g}}_{-5}, \quad\left[\mathfrak{g}_{-2}, V_{1}\right]=\hat{\mathfrak{g}}_{-4} .
$$

Actually, by matrices calculation, we obtain

$$
\left[v_{2}, v_{1}\right]=\left(a_{1} \mathfrak{b}_{1}-a_{2} \mathfrak{b}_{2}\right) \oplus\left(\begin{array}{cc}
{ }^{t} \mathfrak{b}_{2} \mathfrak{b}_{1} & 0 \\
0 & -{ }^{t} \mathfrak{b}_{1} \mathfrak{b}_{2}
\end{array}\right) \in \mathfrak{g}_{-2}, \quad[\xi \oplus C, \eta \oplus B]=\left(\begin{array}{c}
a_{1} c+{ }^{t} \mathfrak{b}_{2} \xi \\
-c a_{2}+{ }^{t} \mathfrak{b}_{1} \xi
\end{array}\right) \in \mathfrak{g}_{-3},
$$

for $v_{2}={ }^{t}\left(0, a_{2}\right) \oplus\left(\mathfrak{b}_{1}, 0\right) \in V_{2}, v_{1}={ }^{t}\left(a_{1}, 0\right) \oplus\left(0, \mathfrak{b}_{2}\right) \in V_{1}$ and for $\xi \oplus C \in \mathfrak{g}_{-2}, \eta \oplus B \in \mathfrak{g}_{-1}$, where $C=\left(\begin{array}{cc}c & 0 \\ 0 & -c\end{array}\right), \eta={ }^{t}\left(a_{1}, a_{2}\right)$ and $B=\left(\mathfrak{b}_{1}, \mathfrak{b}_{2}\right)$. Thus, putting,

$$
\begin{gathered}
Z_{1}={ }^{t}(1,0) \in \hat{\mathfrak{g}}_{-4} \subset \mathfrak{g}_{-3}, \quad Z_{2}={ }^{t}(0,1) \in \hat{\mathfrak{g}}_{-5} \subset \mathfrak{g}_{-3}, \quad Y_{1}=\left(\begin{array}{cc}
1 & 0 \\
0 & -1
\end{array}\right) \in \mathfrak{g}_{-2}, \\
Y_{i+1}=\boldsymbol{e}_{i} \in \mathfrak{g}_{-2}, \quad X_{1}^{1}={ }^{t}(1,0), \quad X_{1}^{i+1}=\left(0, \boldsymbol{e}_{i}\right) \in V_{1}, \quad X_{2}^{1}={ }^{t}(0,-1), \quad X_{2}^{i+1}=\left(\boldsymbol{e}_{i}, 0\right) \in V_{2},
\end{gathered}
$$

for $i=1, \ldots, p$, where $\boldsymbol{e}_{i}$ is the vector in $\mathbb{R}^{p}$, whose $i$-th component is 1 and other components are 0 . We obtain a basis $\left\{Z_{1}, Z_{2}\right\}$ of $\mathfrak{g}_{-3}$, a basis $\left\{Y_{1}, \ldots, Y_{p+1}\right\}$ of $\mathfrak{g}_{-2}$, a basis $\left\{X_{1}^{1}, \ldots, X_{1}^{p+1}\right\}$ of $V_{1}$ and a basis $\left\{X_{2}^{1}, \ldots, X_{2}^{p+1}\right\}$ of $V_{2}$. Moreover we have the bracket relation among these vectors;

$$
\begin{gathered}
Z_{1}=\delta_{j_{1}}^{j_{2}} \cdot\left[Y_{j_{1}}, X_{1}^{j_{2}}\right], \quad Z_{2}=\delta_{j_{1}}^{j_{2}} \cdot\left[Y_{j_{1}}, X_{2}^{j_{2}}\right] \quad Y_{1}=\delta_{k_{1}}^{k_{2}} \cdot\left[X_{2}^{k_{1}}, X_{1}^{k_{2}}\right], \\
Y_{k}=\left[X_{2}^{1}, X_{1}^{k}\right]=\left[X_{2}^{k}, X_{1}^{1}\right], \quad\left[X_{1}^{j_{1}}, X_{1}^{j_{2}}\right]=\left[X_{2}^{j_{1}}, X_{2}^{j_{2}}\right]=0,
\end{gathered}
$$

for $j_{1}, j_{2}=1,2, \ldots, p+1, k_{1}, k_{2}, k=2, \ldots, p+1$. Hence, taking the dual basis $\left\{\hat{\varpi}_{1}, \hat{\varpi}_{2}, \hat{\omega}_{1}, \ldots, \hat{\omega}_{p+1}\right.$, $\left.\hat{\varpi}_{1}^{1} \ldots, \hat{\varpi}_{1}^{p+1}, \hat{\varpi}_{2}^{1}, \ldots, \hat{\varpi}_{2}^{p+1}\right\}$ in $\mathfrak{m}_{3}^{*}$ of the above basis $\left\{Z_{1}, Z_{2}, Y_{1}, \ldots, Y_{p+1}, X_{1}^{1}, \ldots, X_{1}^{p+1}, X_{2}^{1}, \ldots\right.$, 
$\left.X_{2}^{p+1}\right\}$ in $\mathfrak{m}_{3}=\mathfrak{g}_{-3} \oplus \mathfrak{g}_{-2} \oplus \mathfrak{g}_{-1}$, we have the following structure equation of the Lie algebra $\mathfrak{m}_{3}$

$$
\left\{\begin{array}{l}
d \hat{\varpi}_{1}=\hat{\varpi}_{1}^{1} \wedge \hat{\omega}_{1}+\cdots+\hat{\varpi}_{1}^{p+1} \wedge \hat{\omega}_{p+1}, \\
d \hat{\varpi}_{2}=\hat{\varpi}_{2}^{1} \wedge \hat{\omega}_{1}+\cdots+\hat{\varpi}_{2}^{p+1} \wedge \hat{\omega}_{p+1}, \\
d \hat{\omega}_{1}=\hat{\varpi}_{1}^{2} \wedge \hat{\varpi}_{2}^{2}+\cdots+\hat{\varpi}_{1}^{p+1} \wedge \hat{\varpi}_{2}^{p+1}, \\
d \hat{\omega}_{k}=\hat{\varpi}_{1}^{1} \wedge \hat{\varpi}_{2}^{k}+\hat{\varpi}_{1}^{k} \wedge \hat{\varpi}_{2}^{1}, \quad(k=2, \ldots, p+1), \\
d \hat{\varpi}_{1}^{j}=d \hat{\varpi}_{2}^{j}=0 \quad(j=1,2, \ldots, p+1) .
\end{array}\right.
$$

These being prepared, let $(Z, D)$ be a regular differential system of type $\mathfrak{m}_{3}$. Let us fix a point $z \in Z$. Then there exists a coframe $\left\{\varpi_{0}, \varpi_{1}, \omega_{1}, \ldots, \omega_{p+1}, \varpi_{0}^{1}, \ldots, \varpi_{0}^{p+1}, \varpi_{1}^{1}, \ldots, \varpi_{1}^{p+1}\right\}$ defined on a neighborhood $U$ of $z \in Z$ such that

$$
F=\partial D=\left\{\varpi_{0}=\varpi_{1}=0\right\}, \quad D=\left\{\varpi_{0}=\varpi_{1}=\omega_{1}=\cdots=\omega_{p+1}=0\right\},
$$

and that the following holds:

$$
\begin{aligned}
& \left\{\begin{array}{l}
d \varpi_{0} \equiv \varpi_{0}^{1} \wedge \omega_{1}+\cdots+\varpi_{0}^{p+1} \wedge \omega_{p+1}, \\
d \varpi_{1} \equiv \varpi_{1}^{1} \wedge \omega_{1}+\cdots+\varpi_{1}^{p+1} \wedge \omega_{p+1} \quad\left(\bmod \varpi_{0}, \varpi_{1}, \omega_{j_{1}} \wedge \omega_{j_{2}}\left(1 \leqq j_{1}<j_{2} \leqq p+1\right)\right),
\end{array}\right. \\
& \left\{\begin{array}{l}
d \omega_{1} \equiv \varpi_{0}^{2} \wedge \varpi_{1}^{2}+\cdots+\varpi_{0}^{p+1} \wedge \varpi_{1}^{p+1}, \\
d \omega_{k} \equiv \varpi_{0}^{1} \wedge \varpi_{1}^{k}+\varpi_{0}^{k} \wedge \varpi_{1}^{1}, \quad(k=2, \ldots, p+1) \quad\left(\bmod \varpi_{0}, \varpi_{1}, \omega_{1}, \ldots, \omega_{p+1}\right) .
\end{array}\right.
\end{aligned}
$$

Adjusting $\varpi_{0}^{j}, \varpi_{1}^{j}\left(\bmod \omega_{1}, \ldots, \omega_{p+1}\right)$, we actually have

$$
\left\{\begin{array}{l}
d \varpi_{0} \equiv \varpi_{0}^{1} \wedge \omega_{1}+\cdots+\varpi_{0}^{p+1} \wedge \omega_{p+1}, \\
d \varpi_{1} \equiv \varpi_{1}^{1} \wedge \omega_{1}+\cdots+\varpi_{1}^{p+1} \wedge \omega_{p+1} . \quad\left(\bmod \varpi_{0}, \varpi_{1}\right)
\end{array}\right.
$$

This equation describes the structure equation of $(Z, F)$, where $F=\partial D$. Namely $(Z, F)$ is a regular differential system of type $\mathfrak{c}^{1}(p+1,2)$. Hence $D$ is a covariant system of $(Z, F)$ (see Theorem $1.4[22])$. Moreover, by Theorem $6.1,\left(R(Z) ; D_{Z}^{1}, D_{Z}^{2}\right)$ is a $P D$ manifold of second order and, in fact, $\hat{\zeta}(R(Z))$ is an equation of Goursat type [25].

Now we will consider the following submanifold $\bar{Y}=\bar{Y}(Z)$ of $\left(\tilde{Y}(Z) ; F_{N}^{*}, F_{N}\right)$ :

$$
\bar{Y}=\bar{Y}(Z)=\bigcup_{z \in Z} \bar{Y}_{z}, \quad \bar{Y}_{z}=\{y \in \operatorname{Gr}(F(z), 3 p+2) \mid y \supset D(z)\} \cong \mathbb{P}(F(z) / D(z)) \cong \mathbb{P}^{p} .
$$

On $\bar{Y}$, we have a pair of differential systems $D_{N}^{*}$ and $D_{N}$ defined by

$$
D_{N}^{*}(y)=\gamma_{*}^{-1}(F(z)) \supset D_{N}(y)=\gamma_{*}^{-1}(y),
$$

where $\gamma: \bar{Y} \rightarrow Z$ is the projection and $z=\gamma(y) . D_{N}^{*}$ is a lift of $F$ and $D_{N}$ is the canonical system by this Grassmannian construction and is a subbundle of $D_{N}^{*}$ of codimension $1 . D_{N}^{*}$ is a differential system of codimension 2 and its Engel-half rank is $p+1$. Since $D$ is a covariant system of $(Z, F)$, an isomorphism $\varphi:(Z, F) \rightarrow(Z, F)$ induces the isomorphism $\varphi_{1}:\left(\bar{Y} ; D_{N}^{*}, D_{N}\right) \rightarrow$ $\left(\bar{Y} ; D_{N}^{*}, D_{N}\right)$ by $\varphi_{1}(y)=\varphi_{*}(y) \in \operatorname{Gr}(F(\varphi(z)), 3 p+2)$ for $z=\gamma(y)$. Conversely, since $D_{N}$ is the canonical system by the Grassmannian construction and $\operatorname{Ker} \gamma_{*}=\mathrm{Ch}\left(D_{N}^{*}\right)$, an isomorphism $\Phi:\left(\bar{Y} ; D_{N}^{*}, D_{N}\right) \rightarrow\left(\bar{Y} ; D_{N}^{*}, D_{N}\right)$ induces the isomorphism $\varphi:(Z, F) \rightarrow(Z, F)$ such that $\Phi=\varphi_{1}$ (cf. the proof of Theorem $4.1[27]$ ).

We will show that $\left(\bar{Y} ; D_{N}^{*}, D_{N}\right)$ satisfies the conditions $(\hat{Y} .1)$ and $(\hat{Y} .2)$ in $\S 7.2$ with $s=1$, $r=2$ and $t_{1}=p+1$ on an open dense subset $Y$ of $\bar{Y}$. For this purpose, let us introduce a fibre coordinate $\left(\mu_{1}, \ldots, \mu_{p}\right)$ of $\gamma: \bar{Y} \rightarrow Z$ by putting

$$
\varpi_{2}=\omega_{1}+\mu_{1} \omega_{2}+\cdots+\mu_{p} \omega_{p+1},
$$


where

$$
D_{N}^{*}=\left\{\varpi_{0}=\varpi_{1}=0\right\}, \quad \text { and } \quad D_{N}=\left\{\varpi_{0}=\varpi_{1}=\varpi_{2}=0\right\} \quad \text { on } \gamma^{-1}(U) .
$$

Here we denote the pullback on $\bar{Y}$ of 1 -forms on $Z$ by the same symbol. Then, by (8.6), we calculate

$$
\begin{aligned}
d \varpi_{2} & =d \omega_{1}+\sum_{\alpha=1}^{p}\left\{\mu_{\alpha} d \omega_{\alpha+1}+d \mu_{\alpha} \wedge \omega_{\alpha+1}\right\}, \\
& \equiv \sum_{\alpha=1}^{p}\left\{\varpi_{0}^{\alpha+1} \wedge \varpi_{1}^{\alpha+1}+\mu_{\alpha}\left(\varpi_{0}^{1} \wedge \varpi_{1}^{\alpha+1}+\varpi_{0}^{\alpha+1} \wedge \varpi_{1}^{1}\right)+\left(d \mu_{\alpha}+\beta_{\alpha}\right) \wedge \omega_{\alpha+1}\right\}, \\
& \equiv \sum_{\alpha=1}^{p}\left\{\left(\varpi_{0}^{\alpha+1}+\mu_{\alpha} \varpi_{0}^{1}\right) \wedge\left(\varpi_{1}^{\alpha+1}+\mu_{\alpha} \varpi_{1}^{1}\right)+\left(d \mu_{\alpha}+\beta_{\alpha}\right) \wedge \omega_{\alpha+1}\right\}-\left(\sum_{\alpha=1}^{p} \mu_{\alpha}^{2}\right) \varpi_{0}^{1} \wedge \varpi_{1}^{1}, \\
& \left(\bmod \varpi_{0}, \varpi_{1}, \varpi_{2}\right)
\end{aligned}
$$

where $\beta_{1}, \ldots, \beta_{p}$ are 1 -forms on $\gamma^{-1}(U)$. Moreover, from (8.7), we have

$$
\left\{\begin{array}{l}
d \varpi_{0} \equiv\left(\varpi_{0}^{2}-\mu_{1} \varpi_{0}^{1}\right) \wedge \omega_{2}+\cdots+\left(\varpi_{0}^{p+1}-\mu_{p} \varpi_{0}^{1}\right) \wedge \omega_{p+1}, \\
d \varpi_{1} \equiv\left(\varpi_{1}^{2}-\mu_{1} \varpi_{1}^{1}\right) \wedge \omega_{2}+\cdots+\left(\varpi_{1}^{p+1}-\mu_{p} \varpi_{1}^{1}\right) \wedge \omega_{p+1} . \quad\left(\bmod \varpi_{0}, \varpi_{1}, \varpi_{2}\right)
\end{array}\right.
$$

These three equations describes the structure equation of $\left(\bar{Y}, D_{N}\right)$. In particular, we obtain $\mathrm{Ch}\left(D_{N}\right)=\{0\}$ on an open dense subset satisfying $\sum_{\alpha=1}^{p} \mu_{\alpha}^{2} \neq 0$. We put

$$
Y=\left\{y \in \bar{Y} \mid \operatorname{Ch}\left(D_{N}\right)(y)=\{0\}\right\} .
$$

Then, by $(8.9),\left(Y ; D_{N}^{*}, D_{N}\right)$ satisfies the conditions $(\hat{Y} .1)$ and $(\hat{Y} .2)$ in $\S 7.2$ with $s=1, r=2$ and $t_{1}=p+1$.

We will consider now the $I G$ manifold $\left(W(Y) ; C_{Y}^{*}, N_{Y}\right)$ of corank 2 given in Proposition 7.4. Let us consider

$$
\varpi=\varpi_{0}+\lambda \varpi_{1}
$$

on $\hat{U}=\gamma^{-1}(U)$. Namely we consider a point $w \in \tilde{W}(Y)$ such that $w=\{\varpi=0\} \subset T_{y}(Y)$, where $y=\mu(w) \in \hat{U}$. Here $(\lambda)$ constitutes an inhomogeneous coordinate of the fibres of $\mu: \hat{W}(Y) \rightarrow Y$. By (8.7) and substituting $\omega_{1}=\varpi_{2}-\sum_{\alpha=1}^{p} \mu_{\alpha} \omega_{\alpha+1}$, we calculate

$$
\begin{aligned}
d \varpi & =d \varpi_{0}+\lambda d \varpi_{1}+d \lambda \wedge \varpi_{1}, \\
& \equiv \sum_{\alpha=1}^{p+1}\left(\varpi_{0}^{\alpha}+\lambda \varpi_{1}^{\alpha}\right) \wedge \omega_{\alpha}+(d \lambda+\beta) \wedge \varpi_{1}, \quad(\bmod \varpi) \\
& \equiv \omega_{1}^{*} \wedge\left(-\sum_{\alpha=1}^{p} \mu_{\alpha} \omega_{\alpha+1}\right)+\sum_{\alpha=2}^{p+1}\left(\varpi_{0}^{\alpha}+\lambda \varpi_{1}^{\alpha}\right) \wedge \omega_{\alpha}+\omega_{1}^{*} \wedge \varpi_{2}+(d \lambda+\beta) \wedge \varpi_{1}, \\
& \equiv \sum_{\alpha=1}^{p}\left\{\varpi_{0}^{\alpha+1}+\lambda \varpi_{1}^{\alpha+1}-\mu_{\alpha} \omega_{1}^{*}\right\} \wedge \omega_{\alpha+1}+\omega_{1}^{*} \wedge \varpi_{2}+(d \lambda+\beta) \wedge \varpi_{1}, \\
& \equiv \sum_{\alpha=1}^{p} \varpi_{\alpha+2}^{*} \wedge \omega_{\alpha+1}+\omega_{1}^{*} \wedge \varpi_{2}+(d \lambda+\beta) \wedge \varpi_{1}, \quad(\bmod \varpi)
\end{aligned}
$$

for some 1-form $\beta$ on $\mu^{-1}(\hat{U})$, where we put $\omega_{1}^{*}=\varpi_{0}^{1}+\lambda \varpi_{1}^{1}$ and $\varpi_{\alpha+1}^{*}=\varpi_{0}^{\alpha}+\lambda \varpi_{1}^{\alpha}-\mu_{\alpha-1} \omega_{1}^{*}$ for $\alpha=2, \ldots, p+1$. Hence we see $W(Y)=\hat{W}(Y)$. Here, again, we denote the pullback on 
$W(Y)$ of 1-forms on $Y$ by the same symbol. By (8.10), we have

$C_{Y}^{*}=\{\varpi=0\}, \quad \operatorname{Ch}\left(C_{Y}^{*}\right)=\left\{\varpi=\varpi_{1}=\varpi_{2}=\varpi_{\alpha+2}^{*}=d \lambda+\beta=\omega_{1}^{*}=\omega_{\alpha+1}=0(1 \leqq \alpha \leqq p)\right\}$.

Moreover, by (8.9), we have

$$
d \varpi_{1} \equiv\left(\varpi_{1}^{2}-\mu_{1} \varpi_{1}^{1}\right) \wedge \omega_{2}+\cdots+\left(\varpi_{1}^{p+1}-\mu_{p} \varpi_{1}^{1}\right) \wedge \omega_{p+1} \quad\left(\bmod \varpi, \varpi_{1}, \varpi_{2}\right) .
$$

Also, by (8.8), we have

$$
\begin{aligned}
d \varpi_{2} & \equiv \sum_{\alpha=1}^{p}\left\{\left(\varpi_{\alpha+2}^{*}-\lambda \varpi_{1}^{\alpha+1}+\mu_{\alpha} \omega_{1}^{*}+\mu_{\alpha} \varpi_{0}^{1}\right) \wedge\left(\varpi_{1}^{\alpha+1}+\mu_{\alpha} \varpi_{1}^{1}\right)+\left(d \mu_{\alpha}+\beta_{\alpha}\right) \wedge \omega_{\alpha+1}\right\} \\
& -\left(\sum_{\alpha=1}^{p} \mu_{\alpha}^{2}\right) \varpi_{0}^{1} \wedge \varpi_{1}^{1} \quad\left(\bmod \varpi, \varpi_{1}, \varpi_{2}\right), \\
& \equiv \sum_{\alpha=1}^{p}\left\{\varpi_{\alpha+2}^{*} \wedge\left(\varpi_{1}^{\alpha+1}+\mu_{\alpha} \varpi_{1}^{1}\right)+\left(d \mu_{\alpha}+\beta_{\alpha}\right) \wedge \omega_{\alpha+1}\right\}+\omega_{1}^{*} \wedge\left\{\left(\sum_{\alpha=1}^{p} \mu_{\alpha}^{2}\right) \varpi_{1}^{1}+\sum_{\alpha=1}^{p} 2 \mu_{\alpha} \varpi_{1}^{\alpha+1}\right\} \\
& \left(\bmod \varpi, \varpi_{1}, \varpi_{2}\right) .
\end{aligned}
$$

Thus we obtain the following structure equation for $\left(W(Y), N_{Y}\right)$ :

$$
N_{Y}=\left\{\varpi=\varpi_{1}=\varpi_{2}=0\right\}, \quad N_{Y}^{\perp}=\left\{\varpi=\varpi_{1}=\varpi_{2}=\varpi_{\alpha+2}^{*}=\omega_{\alpha+1}=0(1 \leqq \alpha \leqq p)\right\},
$$

$$
\left\{\begin{array}{c}
d \varpi \equiv \sum_{\alpha=1}^{p} \varpi_{\alpha+2}^{*} \wedge \omega_{\alpha+1}, \\
d \varpi_{1} \equiv \sum_{\alpha=1}^{p}\left(\varpi_{1}^{\alpha+1}-\mu_{\alpha} \varpi_{1}^{1}\right) \wedge \omega_{\alpha+1}, \\
d \varpi_{2} \equiv \sum_{\alpha=1}^{p}\left\{\varpi_{\alpha+2}^{*} \wedge\left(\varpi_{1}^{\alpha+1}+\mu_{\alpha} \varpi_{1}^{1}\right)+\left(d \mu_{\alpha}+\beta_{\alpha}\right) \wedge \omega_{\alpha+1}\right\} \\
+\omega_{1}^{*} \wedge\left\{\left(\sum_{\alpha=1}^{p} \mu_{\alpha}^{2}\right) \varpi_{1}^{1}+\sum_{\alpha=1}^{p} 2 \mu_{\alpha} \varpi_{1}^{\alpha+1}\right\}
\end{array}\right.
$$

Utilizing this structure equation, we first calculate, for $X \in \operatorname{Ch}\left(C_{Y}^{*}\right)(w), w \in W(Y)$,

$$
\left.X\rfloor d \varpi \equiv 0, \quad X\rfloor d \varpi_{1} \equiv 0, \quad X\right\rfloor d \varpi_{2} \equiv-\hat{\varpi}_{1}^{1}(X) \omega_{1}^{*} \quad\left(\bmod \quad N_{Y}^{\perp}\right),
$$

where we put $\hat{\varpi}_{1}^{1}=\left(\sum_{\alpha=1}^{p} \mu_{\alpha}^{2}\right) \varpi_{1}^{1}+\sum_{\alpha=1}^{p} 2 \mu_{\alpha} \varpi_{1}^{\alpha+1}$. Hence, by (2.3), we get

$$
S\left(N_{Y}\right)(w)=\left\{X \in \operatorname{Ch}\left(C_{Y}^{*}\right)(w) \mid \hat{\varpi}_{1}^{1}(X)=0\right\} .
$$

Now we consider the Lagrange Grassmann bundle $R(Y)=R(W(Y))$ over $\left(W(Y) ; C_{Y}^{*}, N_{Y}\right)$ :

$$
R(Y)=\bigcup_{w \in W(Y)} R_{w}, \quad R_{w}=\left\{\hat{v} \subset N_{Y}(w)|d \varpi|_{\hat{v}}=0, \quad \hat{v} \text { is maximal }\right\} .
$$

From $N_{Y}^{\perp}(w) \subset \hat{v} \subset N_{Y}(w)$ and $\left.d \varpi\right|_{\hat{v}}=0$, if $\left.(d \lambda+\beta) \wedge \omega_{1}^{*} \wedge \omega_{2} \wedge \cdots \wedge \omega_{p+1}\right|_{\hat{v}} \neq 0$, it follows that

$$
\hat{v}=\left\{X \in N_{Y}(w) \mid \varpi_{\alpha+2}(X)=0 \quad(1 \leqq \alpha \leqq p)\right\},
$$

where $\varpi_{\alpha+2}=\varpi_{\alpha+2}^{*}-\sum_{\beta=1}^{p} p_{\alpha \beta}^{*} \omega_{\beta+1}$ for $1 \leqq \alpha \leqq p$ and $p_{\alpha \beta}^{*}=p_{\beta \alpha}^{*}(1 \leqq \alpha, \beta \leqq p)$. For these $\hat{v} \in R_{w}$, we claim

$$
A(\hat{v})=\{0\} \quad \text { for } \quad \hat{v}=\left\{\varpi=\varpi_{1}=\varpi_{2}=\varpi_{\alpha+2}=0(1 \leqq \alpha \leqq p)\right\} .
$$


In fact, by the structure equation (8.11), we get

$$
\left\{\begin{aligned}
d \varpi & \equiv 0, \quad d \varpi_{1} \equiv \sum_{\alpha=1}^{p}\left(\varpi_{1}^{\alpha+1}-\mu_{\alpha} \varpi_{1}^{1}\right) \wedge \omega_{\alpha+1}, \\
d \varpi_{2} & \equiv \sum_{\alpha=1}^{p}\left\{d \mu_{\alpha}+\beta_{\alpha}-\sum_{\beta=1}^{p} p_{\alpha \beta}^{*}\left(\varpi_{1}^{\beta+1}+\mu_{\beta} \varpi_{1}^{1}\right)\right\} \wedge \omega_{\alpha+1}+\omega_{1}^{*} \wedge \hat{\varpi}_{1}^{1} . \quad\left(\bmod \quad(\hat{v})^{\perp}\right)
\end{aligned}\right.
$$

Hence, for $X \in S\left(N_{Y}\right)(w)$, we have

$$
X\rfloor d \varpi \equiv 0, \quad X\rfloor d \varpi_{1} \equiv \sum_{\alpha=1}^{p}\left(\varpi_{1}^{\alpha+1}-\mu_{\alpha} \varpi_{1}^{1}\right)(X) \omega_{\alpha+1} \quad\left(\bmod \quad(\hat{v})^{\perp}\right),
$$

and

$$
X\rfloor d \varpi_{2} \equiv \sum_{\alpha=1}^{p}\left\{d \mu_{\alpha}+\beta_{\alpha}-\sum_{\beta=1}^{p} p_{\alpha \beta}^{*}\left(\varpi_{1}^{\beta+1}+\mu_{\beta} \varpi_{1}^{1}\right)\right\}(X) \omega_{\alpha+1} \quad\left(\bmod \quad(\hat{v})^{\perp}\right) .
$$

Then, from Lemma 4.2 (2), we obtain

$$
\begin{aligned}
& A(\hat{v})=\left\{X \in S\left(N_{Y}\right)(w) \mid\left(\varpi_{1}^{\alpha+1}-\mu_{\alpha} \varpi_{1}^{1}\right)(X)=\right. \\
&\left.\left\{d \mu_{\alpha}+\beta_{\alpha}-\sum_{\beta=1}^{p} p_{\alpha \beta}^{*}\left(\varpi_{1}^{\beta+1}+\mu_{\beta} \varpi_{1}^{1}\right)\right\}(X)=0 \quad(1 \leqq \alpha \leqq p)\right\} \\
&=\operatorname{Ch}\left(C_{Y}^{*}\right)(w) \cap \operatorname{Ch}\left(N_{Y}\right)(w)=\{0\} .
\end{aligned}
$$

Hence $\left(R(Y) ; D_{Y}^{1}, D_{Y}^{2}\right)$ is a $P D$ manifold of second order on an open subset of $R(Y)$. Moreover, by (8.10) and (8.12), we have the structure equation for $\left(R(Y) ; D_{Y}^{1}, D_{Y}^{2}\right)$ as follows;

$$
\begin{gathered}
D_{Y}^{1}=\{\varpi=0\}, \quad D_{Y}^{2}=\left\{\varpi=\varpi_{1}=\cdots=\varpi_{p+2}=0\right\}, \\
d \varpi \equiv \omega_{0}^{*} \wedge \varpi_{1}+\omega_{1}^{*} \wedge \varpi_{2}+\sum_{\alpha=1}^{p} \varpi_{\alpha+2} \wedge \omega_{\alpha+1}, \quad(\bmod \varpi) \\
\left\{\begin{array}{l}
d \varpi_{1} \equiv \sum_{\alpha=1}^{p} \pi_{1}^{\alpha+2} \wedge \omega_{\alpha+1}, \\
d \varpi_{2} \equiv \pi_{2}^{2} \wedge \omega_{1}^{*}+\sum_{\alpha=1}^{p} \pi_{2}^{\alpha+2} \wedge \omega_{\alpha+1}, \quad\left(\bmod \varpi, \varpi_{1}, \ldots, \varpi_{p+2}\right)
\end{array}\right.
\end{gathered}
$$

where we put $\omega_{0}^{*}=d \lambda+\beta, \pi_{1}^{\alpha+2}=\varpi_{1}^{\alpha+1}-\mu_{\alpha} \varpi_{1}^{1}, \pi_{2}^{2}=-\hat{\varpi}_{1}^{1}$ and $\pi_{2}^{\alpha+2}=d \mu_{\alpha}+\beta_{\alpha}-$ $\sum_{\beta=1}^{p} p_{\alpha \beta}^{*}\left(\varpi_{1}^{\beta+1}+\mu_{\beta} \varpi_{1}^{1}\right)$ for $\alpha=1, \ldots, p$. This shows that $\left(R(Y) ; D_{Y}^{1}, D_{Y}^{2}\right)$ is a $P D$ manifold of second order, which is of type $\mathfrak{s}$, where $\mathfrak{s}$ is given by

$$
\mathfrak{s}=\mathfrak{s}_{-3} \oplus \mathfrak{s}_{-2} \oplus \mathfrak{s}_{-1}, \quad \mathfrak{s}_{-3}=\mathbb{R}, \quad \mathfrak{s}_{-2}=V^{*}, \quad \mathfrak{s}_{-1}=V \oplus \mathfrak{f}, \quad \mathfrak{f} \subset S^{2}\left(V^{*}\right),
$$

such that

$$
(\mathfrak{f})^{\perp}=\left\langle\left\{e_{1} \odot e_{1}, e_{1} \odot e_{2}\right\}\right\rangle,
$$

for a base $\left\{e_{1}, \ldots, e_{p+2}\right\}$ of $V$. Thus $\zeta(R(Y))$ is an involutive system of second order of codimension 2 (see Proposition 3.3 [27]).

To obtain an explicit description of the model equation in this case, we will first construct the standard differential system $\left(M\left(\mathfrak{m}_{3}\right), D_{\mathfrak{m}_{3}}\right)$ of type $\mathfrak{m}_{3}$, where $\mathfrak{m}_{3}=\mathfrak{g}_{-3} \oplus \mathfrak{g}_{-2} \oplus \mathfrak{g}_{-1}$ is the negative part of the simple graded Lie algebra of type $\left(B D_{\ell},\left\{\alpha_{1}, \alpha_{3}\right\}\right)$, by virtue of the formula given by $\mathrm{N}$.Tanaka in $\S 2.3[16]$. Let us take the basis $\left\{Z_{1}, Z_{2}, Y_{j}, X_{1}^{j}, X_{2}^{j}(1 \leqq j \leqq p+1)\right\}$ of 
$\mathfrak{m}_{3}$ as above. We introduce a coordinate system $\left(z^{1}, z^{2}, y^{1}, \ldots, y^{p+1}, x_{1}^{1}, \ldots, x_{p+1}^{1}, x_{1}^{2}, \ldots, x_{p+1}^{2}\right)$ of $\mathfrak{m}_{3}$ by putting

$$
u^{-3}=z^{1} Z_{1}+z^{2} Z_{2}, \quad u^{-2}=y^{1} Y_{1}+\cdots+y^{p+1} Y_{p+1},
$$

and

$$
u^{-1}=x_{1}^{1} X_{1}^{1}+\cdots+x_{p+1}^{1} X_{1}^{p+1}+x_{1}^{2} X_{2}^{1}+\cdots+x_{p+1}^{2} X_{2}^{p+1},
$$

where $u^{p}: \mathfrak{m}_{3}=\mathfrak{g}_{-3} \oplus \mathfrak{g}_{-2} \oplus \mathfrak{g}_{-1} \rightarrow \mathfrak{g}_{p}$ is the projection for $p=-1,-2,-3$. Then, by the formula in $\S 2.3$ [16], we calculate

$$
\begin{aligned}
d u^{-2}-\frac{1}{2}\left[u^{-1}, d u^{-1}\right] & =\sum_{k=1}^{p+1} d y^{k} Y_{k}-\frac{1}{2}\left[\sum_{k=1}^{p+1}\left(x_{k}^{1} X_{1}^{k}+x_{k}^{2} X_{2}^{k}\right), \sum_{k=1}^{p+1}\left(d x_{k}^{1} X_{1}^{k}+d x_{k}^{2} X_{2}^{k}\right)\right], \\
& =\bar{\omega}_{1} Y_{1}+\bar{\omega}_{2} Y_{2}+\cdots+\bar{\omega}_{p+1} Y_{p+1},
\end{aligned}
$$

where

$$
\bar{\omega}_{1}=d y^{1}+\frac{1}{2} \sum_{k=2}^{p+1}\left(x_{k}^{1} d x_{k}^{2}-x_{k}^{2} d x_{k}^{1}\right), \bar{\omega}_{k}=d y^{k}+\frac{1}{2}\left(x_{1}^{1} d x_{k}^{2}-x_{k}^{2} d x_{1}^{1}\right)+\frac{1}{2}\left(x_{k}^{1} d x_{1}^{2}-x_{1}^{2} d x_{k}^{1}\right),
$$

for $k=2, \ldots, p+1$, so that

$$
d \bar{\omega}_{1}=d x_{2}^{1} \wedge d x_{2}^{2}+\cdots+d x_{p+1}^{1} \wedge d x_{p+1}^{2}, \quad d \bar{\omega}_{k}=d x_{1}^{1} \wedge d x_{k}^{2}+d x_{k}^{1} \wedge d x_{1}^{2} \quad(k=2, \ldots, p+1) .
$$

Moreover, we calculate

$$
\begin{aligned}
d u^{-3}- & \frac{1}{3}\left[u^{-2}, d u^{-1}\right]-\frac{2}{3}\left[u^{-1}, d u^{-2}\right]+\frac{1}{6}\left[u^{-1},\left[u^{-1}, d u^{-1}\right]\right] \\
= & d z^{1} Z_{1}+d z^{2} Z_{2} \\
& -\frac{1}{3}\left[\sum_{k=1}^{p+1} y^{k} Y_{k}, \sum_{k=1}^{p+1}\left(d x_{k}^{1} X_{1}^{k}+d x_{k}^{2} X_{2}^{k}\right)\right]-\frac{2}{3}\left[\sum_{k=1}^{p+1}\left(x_{k}^{1} X_{1}^{k}+x_{k}^{2} X_{2}^{k}, \sum_{k=1}^{p+1} d y^{k} Y_{k}\right]\right. \\
& +\frac{1}{6}\left[\sum_{k=1}^{p+1}\left(x_{k}^{1} X_{1}^{k}+x_{k}^{2} X_{2}^{k}\right),\left[\sum_{k=1}^{p+1}\left(x_{k}^{1} X_{1}^{k}+x_{k}^{2} X_{2}^{k}\right), \sum_{k=1}^{p+1}\left(d x_{k}^{1} X_{1}^{k}+d x_{k}^{2} X_{2}^{k}\right)\right]\right] \\
= & \bar{\varpi}_{1} Z_{1}+\bar{\varpi}_{2} Z_{2}
\end{aligned}
$$

where

$$
\left\{\begin{array}{l}
\bar{\varpi}_{1}=d \hat{z}^{1}-\left(y^{1}+\frac{1}{2} \sum_{k=2}^{p+1} x_{k}^{1} x_{k}^{2}\right) d x_{1}^{1}-\sum_{k=2}^{p+1}\left\{y^{k}+\frac{1}{2}\left(x_{1}^{1} x_{k}^{2}+x_{k}^{1} x_{1}^{2}\right)\right\} d x_{k}^{1}, \\
\bar{\varpi}_{2}=d \hat{z}^{2}-\left(y^{1}-\frac{1}{2} \sum_{k=2}^{p+1} x_{k}^{1} x_{k}^{2}\right) d x_{1}^{2}-\sum_{k=2}^{p+1}\left\{y^{k}-\frac{1}{2}\left(x_{1}^{1} x_{k}^{2}+x_{k}^{1} x_{1}^{2}\right)\right\} d x_{k}^{2},
\end{array}\right.
$$

and

so that

$$
\left\{\begin{array}{l}
\hat{z}^{1}=z^{1}+\frac{2}{3} \sum_{k=1}^{p+1} x_{k}^{1} y^{k}+\frac{1}{3} \sum_{k=2}^{p+1} x_{1}^{1} x_{k}^{1} x_{k}^{2}+\frac{1}{6} \sum_{k=2}^{p+1}\left(x_{k}^{1}\right)^{2} x_{1}^{2} \\
\hat{z}^{2}=z^{2}+\frac{2}{3} \sum_{k=1}^{p+1} x_{k}^{2} y^{k}-\frac{1}{3} \sum_{k=2}^{p+1} x_{1}^{2} x_{k}^{1} x_{k}^{2}-\frac{1}{6} \sum_{k=2}^{p+1} x_{1}^{1}\left(x_{k}^{2}\right)^{2}
\end{array}\right.
$$

$$
d \bar{\varpi}_{1}=d x_{1}^{1} \wedge \hat{\omega}_{1}+\cdots+d x_{p+1}^{1} \wedge \hat{\omega}_{p+1}, \quad d \bar{\varpi}_{2}=d x_{1}^{2} \wedge \hat{\omega}_{1}+\cdots+d x_{p+1}^{2} \wedge \hat{\omega}_{p+1} .
$$


Thus, $M\left(\mathfrak{m}_{3}\right) \cong \mathfrak{m}_{3}$ is endowed with a coordinate $\left(\hat{z}^{1}, \hat{z}^{2}, y^{1}, \ldots, y^{p+1}, x_{1}^{1}, \ldots, x_{p+1}^{1}, x_{1}^{2}, \ldots, x_{p+1}^{2}\right)$ such that $D_{\mathfrak{m}_{3}}$ and $\partial D_{\mathfrak{m}_{3}}$ are given by

$$
D_{\mathfrak{m}_{3}}=\left\{\bar{\varpi}_{1}=\bar{\varpi}_{2}=\bar{\omega}_{1}=\cdots=\bar{\omega}_{p+1}=0\right\}, \quad \text { and } \quad \partial D_{\mathfrak{m}_{3}}=\left\{\bar{\varpi}_{1}=\bar{\varpi}_{2}=0\right\} .
$$

Now put $(Z, D)=\left(M\left(\mathfrak{m}_{3}\right), D_{\mathfrak{m}_{3}}\right)$ and $F=\partial D_{\mathfrak{m}_{3}}$.

$$
F=\left\{\varpi_{0}=\varpi_{1}=0\right\}, \quad D=\left\{\varpi_{0}=\varpi_{1}=\omega_{1}=\cdots=\omega_{p+1}\right\},
$$

where $\varpi_{0}=\bar{\varpi}_{1}, \varpi_{1}=\bar{\varpi}_{2}$ and $\omega_{j}=\bar{\omega}_{j}(j=1, \ldots, p+1)$. Let $\bar{Y}=\bar{Y}(Z)$ be the projective bundle over $Z$ and $\gamma: \bar{Y} \rightarrow Z$ be the projection. We introduce a fibre coordinate $\left(\mu_{1}, \ldots, \mu_{p}\right)$ of $\gamma: \bar{Y} \rightarrow Z$ by putting

$$
\varpi_{2}=\omega_{1}+\mu_{1} \omega_{2}+\cdots+\mu_{p} \omega_{p+1}
$$

Then we have

$$
D_{N}^{*}=\left\{\varpi_{0}=\varpi_{1}=0\right\} \quad \text { and } \quad D_{N}=\left\{\varpi_{0}=\varpi_{1}=\varpi_{2}=0\right\} .
$$

Here we denote the pullback on $\bar{Y}$ of 1 -forms on $Z$ by the same symbol. We put

$$
Y=\left\{y \in \bar{Y} \mid \operatorname{Ch}\left(D_{N}\right)(y)=\{0\}\right\} .
$$

Starting from $\left(Y ; D_{N}^{*}, D_{N}\right)$, we construct the $I G$ manifold $\left(W(Y) ; C_{Y}^{*}, N_{Y}\right)$ of corank 2 and the Lagrange Grassmann bundle $\left(R(Y) ; D_{Y}^{1}, D_{Y}^{2}\right)$. We introduce a fibre coordinate $(\lambda)$ of $\mu$ : $W(Y) \rightarrow Y$ and calculate

$$
\begin{aligned}
& \varpi=\varpi_{0}+\lambda \varpi_{1} \\
& =d \hat{z}^{1}-\left(y^{1}+\frac{1}{2} \sum_{k=2}^{p+1} x_{k}^{1} x_{k}^{2}\right) d x_{1}^{1}-\sum_{k=2}^{p+1}\left\{y^{k}+\frac{1}{2}\left(x_{1}^{1} x_{k}^{2}+x_{k}^{1} x_{1}^{2}\right)\right\} d x_{k}^{1} \\
& +\lambda\left[d \hat{z}^{2}-\left(y^{1}-\frac{1}{2} \sum_{k=2}^{p+1} x_{k}^{1} x_{k}^{2}\right) d x_{1}^{2}-\sum_{k=2}^{p+1}\left\{y^{k}-\frac{1}{2}\left(x_{1}^{1} x_{k}^{2}+x_{k}^{1} x_{1}^{2}\right)\right\} d x_{k}^{2}\right] \\
& =d \hat{z}^{1}+\lambda d \hat{z}^{2}-\left(y^{1}+\frac{1}{2} \sum_{k=2}^{p+1} x_{k}^{1} x_{k}^{2}\right)\left(d x_{1}^{1}+\lambda d x_{1}^{2}\right)-\sum_{k=2}^{p+1}\left\{y^{k}+\frac{1}{2}\left(x_{1}^{1} x_{k}^{2}+x_{k}^{1} x_{1}^{2}\right)\right\}\left(d x_{k}^{1}+\lambda d x_{k}^{2}\right) \\
& +\lambda\left\{\left(\sum_{k=2}^{p+1} x_{k}^{1} x_{k}^{2}\right) d x_{1}^{2}+\sum_{k=2}^{p+1}\left(x_{1}^{1} x_{k}^{2}+x_{k}^{1} x_{1}^{2}\right) d x_{k}^{2}\right\} \\
& =d\left(\hat{z}^{1}+\lambda \hat{z}^{2}\right)-\left(y^{1}+\frac{1}{2} \sum_{k=2}^{p+1} x_{k}^{1} x_{k}^{2}\right) d\left(x_{1}^{1}+\lambda x_{1}^{2}\right)-\sum_{k=2}^{p+1}\left\{y^{k}+\frac{1}{2}\left(x_{1}^{1} x_{k}^{2}+x_{k}^{1} x_{1}^{2}\right)\right\} d\left(x_{k}^{1}+\lambda x_{k}^{2}\right) \\
& -\left[\hat{z}^{2}-\left(y^{1}+\frac{1}{2} \sum_{k=2}^{p+1} x_{k}^{1} x_{k}^{2}\right) x_{1}^{2}-\sum_{k=2}^{p+1}\left\{y^{k}+\frac{1}{2}\left(x_{1}^{1} x_{k}^{2}+x_{k}^{1} x_{1}^{2}\right)\right\} x_{k}^{2}\right] d \lambda \\
& +\lambda\left\{d\left(\sum_{k=2}^{p+1} x_{1}^{2} x_{k}^{1} x_{k}^{2}\right)-\sum_{k=2}^{p+1} x_{1}^{2} x_{k}^{2} d x_{k}^{1}+\sum_{k=2}^{p+1} x_{1}^{1} x_{k}^{2} d x_{k}^{2}\right\} \\
& =d \hat{Z}-\sum_{k=1}^{p+1} Y^{k} d\left(x_{k}^{1}+\lambda x_{k}^{2}\right)-P_{1} d \lambda=d Z-\sum_{k=1}^{p+2} P_{k} d X_{k},
\end{aligned}
$$


where

$$
\left\{\begin{array}{c}
\hat{Z}=\hat{z}^{1}+\lambda\left\{\hat{z}^{2}+\sum_{k=2}^{p+1} x_{1}^{2} x_{k}^{1} x_{k}^{2}+\frac{1}{2}\left(x_{1}^{1}+\lambda x_{1}^{2}\right) \sum_{k=2}^{p+1}\left(x_{k}^{2}\right)^{2}\right\}, \quad Y^{1}=y^{1}+\frac{1}{2} \sum_{k=2}^{p+1}\left(x_{k}^{1}+\lambda x_{k}^{2}\right) x_{k}^{2}, \\
Y^{k}=y^{k}+\frac{1}{2}\left\{\left(x_{1}^{1}+\lambda x_{1}^{2}\right) x_{k}^{2}+\left(x_{k}^{1}+\lambda x_{k}^{2}\right) x_{1}^{2}\right\}, \quad P_{1}=\hat{z}^{2}-\sum_{k=1}^{p+1} y^{k} x_{k}^{2}-\frac{1}{2} \lambda x_{1}^{2} \sum_{k=2}^{p+1}\left(x_{k}^{2}\right)^{2},
\end{array}\right.
$$

for $k=2, \ldots, p+1$. Thus, putting

$$
\left\{\begin{array}{l}
Z=\hat{Z}-\sum_{k=2}^{p+1} Y^{k}\left(x_{k}^{1}+\lambda x_{k}^{2}\right), X_{1}=\lambda, X_{2}=x_{1}^{1}+\lambda x_{1}^{2}, P_{2}=Y^{1}, \\
X_{k+1}=Y^{k}, P_{k+1}=-\left(x_{k}^{1}+\lambda x_{k}^{2}\right) \quad(k=2, \ldots, p+1),
\end{array}\right.
$$

we obtain a canonical coordinate $\left(X_{1}, \ldots, X_{p+2}, Z, P_{1}, \ldots, P_{p+2}\right)$ of $J=W(Y) / \mathrm{Ch}\left(C_{Y}^{*}\right)=$ $W(Z) / \mathrm{Ch}\left(C_{Z}^{*}\right)$.

Conversely we calculate

$$
\left\{\begin{array}{l}
x_{1}^{2}=c_{1}, x_{k}^{2}=c_{k}, \lambda=X_{1}, x_{1}^{1}=X_{2}-c_{1} X_{1}, x_{k}^{1}=-\left(P_{k+1}+c_{k} X_{1}\right), \\
y^{1}=P_{2}+\frac{1}{2} \sum_{k=2}^{p+1} c_{k} P_{k+1}, y^{k}=X_{k+1}+\frac{1}{2}\left(c_{1} P_{k+1}-c_{k} X_{2}\right), \quad(k=2, \ldots, p+1) \\
\hat{z}^{2}=P_{1}+c_{1} P_{2}+\sum_{k=2}^{p+1}\left(X_{k+1}+c_{1} P_{k+1}\right) c_{k}+\frac{1}{2}\left(c_{1} X_{1}-X_{2}\right)\left(\sum_{k=2}^{p+1} c_{k}^{2}\right) .
\end{array}\right.
$$

Hence, by (8.13), (8.14) and (8.15), we have

$$
\left\{\begin{array}{c}
\varpi_{1}=d P_{1}+c_{1}\left\{d P_{2}+\sum_{k=2}^{p+1} c_{k} d P_{k+1}+\frac{1}{2}\left(\sum_{k=2}^{p+1} c_{k}^{2}\right) d X_{1}\right\}-\frac{1}{2}\left(\sum_{k=2}^{p+1} c_{k}^{2}\right) d X_{2}+\sum_{k=2}^{p+1} c_{k} d X_{k+1} \\
\varpi_{2}=d P_{2}+\sum_{k=2}^{p+1}\left(c_{k}+c_{1} \mu_{k-1}\right) d P_{k+1}+\frac{1}{2} \sum_{k=2}^{p+1}\left(c_{k}+2 c_{1} \mu_{k-1}\right) c_{k} d X_{1} \\
-\left(\sum_{k=2}^{p+1} c_{k} \mu_{k-1}\right) d X_{2}+\sum_{k=2}^{p+1} \mu_{k-1} d X_{k+1} .
\end{array}\right.
$$

Here $\left(X_{1}, \ldots, X_{p+2}, Z, P_{1}, \ldots, P_{p+2}, c_{1}, \ldots, c_{p+1}, \mu_{1}, \ldots, \mu_{p}\right)$ constitute a coordinate of $W(Y)$ and $\left(X_{1}, \ldots, X_{p+2}, Z, P_{1}, \ldots, P_{p+2}, c_{1}, \ldots, c_{p+1}\right)$ constitutes a coordinate of $W(Z)$. Now, from the canonical coordinate $\left(X_{1}, \ldots, X_{p+2}, Z, P_{1}, \ldots, P_{p+2}\right)$ of $J$, we introduce the coordinate $\left(X_{i}, X_{\alpha}, Z, P_{i}, P_{\alpha}, A_{i}^{\alpha}, B_{i}^{\alpha}, S_{i j}\right)(1 \leqq i \leqq j \leqq 2,3 \leqq \alpha \leqq p+2)$ of $I^{2}(J)$ as in $\S 2.1$. First we calculate

$$
\begin{array}{rl}
\varpi_{1}^{*}=\varpi_{1}-c_{1} \varpi_{2}=d P_{1}-\sum_{k=2}^{p+1} c_{1}^{2} \mu_{k-1} & d P_{k+1}+\sum_{k=2}^{p+1}\left(c_{k}-c_{1} \mu_{k-1}\right) d X_{k+1} \\
& -\sum_{k=2}^{p+1} c_{1}^{2} c_{k} \mu_{k-1} d X_{1}-\frac{1}{2} \sum_{k=2}^{p+1}\left(c_{k}-2 c_{1} \mu_{k-1}\right) c_{k} d X_{2} .
\end{array}
$$

Then, since $N_{Y}=\left\{\varpi=\varpi_{1}^{*}=\varpi_{2}=0\right\}$, the canonical inclusion $\iota: W(Y) \rightarrow I^{2}(J)$ is given by

$$
A_{1}^{\alpha}=c_{1}^{2} \mu_{\alpha-2}, B_{1}^{\alpha}=-\left(c_{\alpha-1}-c_{1} \mu_{\alpha-2}\right), A_{2}^{\alpha}=-\left(c_{\alpha-1}+c_{1} \mu_{\alpha-2}\right), B_{2}^{\alpha}=-\mu_{\alpha-2},
$$




$$
S_{11}=c_{1}^{3} \sum_{k=1}^{p} \mu_{k}^{2}, S_{12}=\frac{1}{2} \sum_{k=1}^{p+1} c_{k}^{2}-c_{1} \sum_{k=2}^{p+1} c_{k} \mu_{k-1}-c_{1}^{2} \sum_{k=1}^{p} \mu_{k}^{2}, S_{22}=c_{1} \sum_{k=1}^{p} \mu_{k}^{2}+2 \sum_{k=2}^{p+1} c_{k} \mu_{k-1} .
$$

Thus, by (4.3), introducing a coordinate $\left(X_{1}, \ldots, X_{p+2}, Z, P_{1}, \ldots, P_{p+2}, c_{1}, \ldots, c_{p+1}, \mu_{1}, \ldots, \mu_{p}, P_{\alpha, \beta}^{*}\right)$ $(3 \leqq \alpha \leqq \beta \leqq p+2)$ of $R(Y)$ as in $\S 4$.1, the immersion $\zeta: R(Y) \rightarrow L(J)$ is expressed by

$$
\left\{\begin{array}{l}
\zeta^{*} P_{\alpha \beta}=P_{\alpha \beta}^{*}, \quad \zeta^{*} P_{\alpha 1}=-\left(c_{\alpha-1}-c_{1} \mu_{\alpha-2}\right)+c_{1}^{2} \sum_{\beta=3}^{p+2} P_{\alpha \beta}^{*} \mu_{\beta-2}, \quad(3 \leqq \alpha \leqq \beta \leqq p+2) \\
\zeta^{*} P_{\alpha 2}=-\mu_{\alpha-2}-\sum_{\beta=3}^{p+2} P_{\alpha \beta}^{*}\left(c_{\beta-1}+c_{1} \mu_{\beta-2}\right), \quad \zeta^{*} P_{11}=c_{1}^{3} \sum_{k=1}^{p} \mu_{k}^{2}+c_{1}^{4} \sum_{\alpha, \beta=3}^{p+2} P_{\alpha \beta}^{*} \mu_{\alpha-2} \mu_{\beta-2}, \\
\zeta^{*} P_{12}=\frac{1}{2} \sum_{k=1}^{p+1} c_{k}^{2}-c_{1} \sum_{k=2}^{p+1} c_{k} \mu_{k-1}-c_{1}^{2} \sum_{k=1}^{p} \mu_{k}^{2}-c_{1}^{2} \sum_{\alpha, \beta=3}^{p+2} P_{\alpha \beta}^{*} \mu_{\alpha-2}\left(c_{\beta-1}+c_{1} \mu_{\beta-2}\right), \\
\zeta^{*} P_{22}=c_{1} \sum_{k=1}^{p} \mu_{k}^{2}+2 \sum_{k=2}^{p+1} c_{k} \mu_{k-1}+\sum_{\alpha, \beta=3}^{p+2} P_{\alpha \beta}^{*}\left(c_{\alpha-1}+c_{1} \mu_{\alpha-2}\right)\left(c_{\beta-1}+c_{1} \mu_{\beta-2}\right) .
\end{array}\right.
$$

This is the description of the model involutive system of codimension 2 of type $\left(B D_{\ell},\left\{\alpha_{1}, \alpha_{3}\right\}\right)$.

Moreover, from the canonical coordinate $\left(X_{1}, \ldots, X_{p+2}, Z, P_{1}, \ldots, P_{p+2}\right)$ of $J$, we introduce the coordinate $\left(X_{1}, X_{a}, Z, P_{1}, P_{a}, \hat{A}_{1}^{a}, \hat{B}_{1}^{a}, \hat{S}_{11}\right)(2 \leqq a \leqq p+2)$ of $I^{1}(J)$ as in $\S 2.1$. Then, from the first equation of (8.16), since $N_{Z}=\left\{\varpi=\varpi_{1}=0\right\}$, the canonical inclusion $\iota: W(Z) \rightarrow I^{1}(J)$ is given by

$$
\hat{A}_{1}^{2}=-c_{1}, \quad \hat{A}_{1}^{a}=-c_{1} c_{a-1}, \quad \hat{B}_{1}^{2}=\frac{1}{2}\left(\sum_{k=2}^{p+1} c_{k}^{2}\right), \quad \hat{B}_{1}^{a}=-c_{a-1}, \quad \hat{S}_{11}=0 .
$$

Thus, by (4.3), introducing a coordinate $\left(X_{1}, \ldots, X_{p+2}, Z, P_{1}, \ldots, P_{p+2}, c_{1}, \ldots, c_{p+1}, P_{a b}^{*}\right)(2 \leqq$ $a \leqq b \leqq p+2)$ of $R(Z)$ as in $\S 4.1$, the immersion $\zeta: R(Z) \rightarrow L(J)$ is expressed by

$$
\left\{\begin{array}{l}
\zeta^{*} P_{a b}=P_{a b}^{*}(2 \leqq a \leqq b \leqq p+2), \quad \zeta^{*} P_{a 1}=-c_{a-1}-c_{1}\left(P_{a 2}^{*}+\sum_{b=3}^{p+2} P_{a b}^{*} c_{b-1}\right) \quad(3 \leqq a \leqq p+2), \\
\zeta^{*} P_{21}=\frac{1}{2}\left(\sum_{k=2}^{p+1} c_{k}^{2}\right)-c_{1}\left(P_{22}^{*}+\sum_{b=3}^{p+2} P_{2 b}^{*} c_{b-1}\right), \zeta^{*} P_{11}=c_{1}^{2}\left(P_{22}^{*}+2 \sum_{a=3}^{p+2} P_{2 a}^{*} c_{a-1}+\sum_{a, b=3}^{p+2} P_{a b}^{*} c_{a-1} c_{b-1}\right) .
\end{array}\right.
$$

This is the description of the model Goursat type equation of type $\left(B D_{\ell},\left\{\alpha_{1}, \alpha_{3}\right\}\right)$ (see Remark $6.2(1))$.

8.4. Other Examples. In this subsection, we will treat the case of Proposition 7.2. For this purpose, we will here exhibit an example of type $\left(C_{3},\left\{\alpha_{2}\right\}\right)$.

From $\S 5[12]$, the structure of the symbol algebra $\mathfrak{m}=\mathfrak{g}_{-2} \oplus \mathfrak{g}_{-1}$ of type $\left(C_{3},\left\{\alpha_{2}\right\}\right)$ can be described as follows; Let $V$ be a vector space of dimension 2 and $(U,\langle\rangle$,$) be a symplectic vector$ space of dimension 2. Then $\mathfrak{m}$ is isomorphic to $\mathfrak{m}^{2}(U, V)$,

$$
\mathfrak{m}^{2}(U, V)=\mathfrak{g}_{-2} \oplus \mathfrak{g}_{-1}, \quad \mathfrak{g}_{-2}=S^{2}(V), \mathfrak{g}_{-1}=U \otimes V .
$$

The bracket product is defined by

$$
\left[u_{1} \otimes v_{1}, u_{2} \otimes v_{2}\right]=\left\langle u_{1}, u_{2}\right\rangle v_{1} \odot v_{2} \quad \text { for } \quad u_{1}, u_{2} \in U, v_{1}, v_{2} \in V .
$$


Moreover the standard differential system $\left(M(\mathfrak{m}), D_{\mathfrak{m}}\right)$ of type $\mathfrak{m}$ in this case is given as follows; $M(\mathfrak{m})=\mathbb{R}^{7}$ is endowed with a coordinate $\left(x_{1}, x_{2}, x_{3}, x_{4}, y_{1}, y_{2}, y_{3}\right)$ such that $D_{\mathfrak{m}}$ is given by

$$
D_{\mathfrak{m}}=\left\{\bar{\theta}_{1}=\bar{\theta}_{2}=\bar{\theta}_{3}=0\right\},
$$

where

$$
\bar{\theta}_{1}=d y_{1}+x_{4} d x_{1}+x_{3} d x_{2}, \quad \bar{\theta}_{2}=d y_{2}+x_{3} d x_{1} \quad \text { and } \quad \bar{\theta}_{3}=d y_{3}+x_{4} d x_{2} .
$$

Thus the symbol algebra $\mathfrak{m}=\mathfrak{g}_{-2} \oplus \mathfrak{g}_{-1} \cong \mathfrak{m}^{2}(U, V)$ is described by

$$
\left\{\begin{array}{l}
d \theta_{1} \equiv \omega_{4} \wedge \omega_{1}+\omega_{3} \wedge \omega_{2} \\
d \theta_{2} \equiv \omega_{3} \wedge \omega_{1} \quad\left(\bmod \quad \theta_{1}, \theta_{2}, \theta_{3}\right) \\
d \theta_{3} \equiv \omega_{4} \wedge \omega_{2}
\end{array}\right.
$$

In fact, taking the dual basis $\left\{\frac{\partial}{\partial y_{1}}, \frac{\partial}{\partial y_{2}}, \frac{\partial}{\partial y_{3}}, X_{1}, X_{2}, X_{3}, X_{4}\right\}$ of the coframe $\left\{\bar{\theta}_{1}, \bar{\theta}_{2}, \bar{\theta}_{3}, d x_{1}, d x_{2}\right.$, $\left.d x_{3}, d x_{4}\right\}$ on $M(\mathfrak{m})$, we have

$$
X_{1}=\frac{\partial}{\partial x_{1}}-x_{4} \frac{\partial}{\partial y_{1}}-x_{3} \frac{\partial}{\partial y_{2}}, \quad X_{2}=\frac{\partial}{\partial x_{2}}-x_{3} \frac{\partial}{\partial y_{1}}-x_{4} \frac{\partial}{\partial y_{3}}, \quad \text { and } \quad X_{3}=\frac{\partial}{\partial x_{3}}, X_{4}=\frac{\partial}{\partial x_{4}} .
$$

Thus $\left\{X_{1}, X_{2}, X_{3}, X_{4}\right\}$ constitutes a free basis of the sections $\Gamma\left(D_{\mathfrak{m}}\right)$ of $D_{\mathfrak{m}}$, and we obtain

$$
\left[X_{2}, X_{3}\right]=\left[X_{1}, X_{4}\right]=\frac{\partial}{\partial y_{1}},\left[X_{1}, X_{3}\right]=\frac{\partial}{\partial y_{2}},\left[X_{2}, X_{4}\right]=\frac{\partial}{\partial y_{3}},\left[X_{1}, X_{2}\right]=\left[X_{3}, X_{4}\right]=0
$$

Here, for a basis $\left\{v_{1}, v_{2}\right\}$ of $V$ and a symplectic basis $\left\{u_{1}, u_{2}\right\}$ of $U, X_{1}, X_{2}, X_{3}$ and $X_{4}$ correspond to $u_{1} \otimes v_{1}, u_{1} \otimes v_{2}, u_{2} \otimes v_{1}$ and $u_{2} \otimes v_{2}$ respectively. Moreover $\frac{\partial}{\partial y_{1}}, \frac{\partial}{\partial y_{2}}$ and $\frac{\partial}{\partial y_{3}}$ correspond to $v_{1} \odot v_{2}, v_{1} \odot v_{1}$ and $v_{2} \odot v_{2}$ respectively. Thus $\mathfrak{m}$ is isomorphic to $\mathfrak{m}^{2}(U, V)$.

Let $\left(Y, D_{N}\right)$ be a regular differential system of type $\mathfrak{m}$ such that $D_{N}$ is locally defined by

$$
D_{N}=\left\{\theta_{1}=\theta_{2}=\theta_{3}=0\right\},
$$

Here $\left\{\theta_{1}, \theta_{2}, \theta_{3}, \omega_{1}, \omega_{2}, \omega_{3}, \omega_{4}\right\}$ forms a coframe on $Y$ satisfying (8.17).

Then, putting $\varpi=\theta_{1}+\lambda_{1} \theta_{2}+\lambda_{2} \theta_{3}$, we calculate

$$
\begin{aligned}
d \varpi & \equiv \omega_{4} \wedge \omega_{1}+\omega_{3} \wedge \omega_{2}+\lambda_{1} \omega_{3} \wedge \omega_{1}+\lambda_{2} \omega_{4} \wedge \omega_{2}+\left(d \lambda_{1}+\gamma_{1}\right) \wedge \theta_{2}+\left(d \lambda_{2}+\gamma_{2}\right) \wedge \theta_{3} \\
& \equiv\left(\omega_{4}+\lambda_{1} \omega_{3}\right) \wedge \omega_{1}+\left(\omega_{3}+\lambda_{2} \omega_{4}\right) \wedge \omega_{2}+\left(d \lambda_{1}+\gamma_{1}\right) \wedge \theta_{2}+\left(d \lambda_{2}+\gamma_{2}\right) \wedge \theta_{3} \\
& \equiv \tilde{\omega}_{4} \wedge \omega_{1}+\tilde{\omega}_{3} \wedge \omega_{2}+\left(d \lambda_{1}+\gamma_{1}\right) \wedge \theta_{2}+\left(d \lambda_{2}+\gamma_{2}\right) \wedge \theta_{3} \quad(\bmod \varpi)
\end{aligned}
$$

for some 1 -forms $\gamma_{1}, \gamma_{2}$ on $Y$, where we put $\tilde{\omega}_{4}=\omega_{4}+\lambda_{1} \omega_{3}$ and $\tilde{\omega}_{3}=\omega_{3}+\lambda_{2} \omega_{4}$. Thus we see

$$
W(Y)=\left\{w \in \hat{W}(Y) \mid \lambda_{1} \lambda_{2}-1 \neq 0\right\}
$$

and we have on $W(Y)$,

$C_{Y}^{*}=\{\varpi=o\}, \quad N_{Y}=\left\{\varpi=\theta_{2}=\theta_{3}=o\right\}, \quad N_{Y}^{\perp}=\left\{\varpi=\theta_{2}=\theta_{3}=\omega_{1}=\omega_{2}=\tilde{\omega}_{3}=\tilde{\omega}_{4}=o\right\}$.

Here $r=2, t=2, n=r+t=4$ and $\operatorname{dim} W(Y)=9$. We see that $\left(Y, D_{N}\right)$ satisfies the condition $(Y .2)$ in $\S 7.1$ and $\left(W(Y), C_{Y}^{*}\right)$ is a contact manifold of dimension 9 . Let $\left(R(Y) ; D_{Y}^{1}, D_{Y}^{2}\right)$ be the Lagrange Grassmann bundle over $\left(W(Y) ; C_{Y}^{*}, N_{Y}\right)$. Then, by Proposition $7.2,\left(R(Y) ; D_{Y}^{1}, D_{Y}^{2}\right)$ is a $P D$ manifold of second order, globally over $W(Y)$. Put

$$
\hat{v}=\left\{X \in N_{Y}(w) \mid \varpi_{3}(X)=\varpi_{4}(X)=0\right\} \in R_{w}(Y),
$$

where $\varpi_{3}=\tilde{\omega}_{3}-r \omega_{2}-s \omega_{1}$ and $\varpi_{4}=\tilde{\omega}_{4}-s \omega_{2}-t \omega_{1}$. Here $(r, s, t)$ is a fibre coordinate of $R_{w}(Y)$. Hence, around $\hat{v} \in R(Y)$, we have

$$
D_{Y}^{2}=\left\{\varpi=\theta_{2}=\theta_{3}=\varpi_{3}=\varpi_{4}=0\right\} .
$$


Then, from

$$
\left\{\begin{array}{l}
\varpi_{3}-\lambda_{2} \varpi_{4}=\left(1-\lambda_{1} \lambda_{2}\right) \omega_{3}-\left(r \omega_{2}+s \omega_{1}\right)-\lambda_{2}\left(s \omega_{2}+t \omega_{1}\right), \\
\varpi_{4}-\lambda_{2} \varpi_{3}=\left(1-\lambda_{1} \lambda_{2}\right) \omega_{4}-\left(s \omega_{2}+t \omega_{1}\right)-\lambda_{1}\left(r \omega_{2}+s \omega_{1}\right),
\end{array}\right.
$$

and by (8.17), we have

$$
\left\{\begin{array}{l}
d \varpi \equiv 0 \\
d \theta_{2} \equiv\left(1-\lambda_{1} \lambda_{2}\right)^{-1}\left(r+\lambda_{2} s\right) \omega_{2} \wedge \omega_{1} \quad\left(\bmod \quad \varpi, \theta_{2}, \theta_{3}, \varpi_{3}, \varpi_{4}\right) \\
d \theta_{3} \equiv\left(1-\lambda_{1} \lambda_{2}\right)^{-1}\left(t+\lambda_{1} s\right) \omega_{1} \wedge \omega_{2}
\end{array}\right.
$$

This shows that $\left(R(Y) ; D_{Y}^{1}, D_{Y}^{2}\right)$ does not satisfy the compatibility condition $(C)$ in $\S 3.1$ on an open subset.

To obtain an explicit description of the model equation in this case, we calculate

$$
\begin{aligned}
\varpi & =d y_{1}+x_{4} d x_{1}+x_{3} d x_{2}+\lambda_{1}\left(d y_{2}+x_{3} d x_{1}\right)+\lambda_{2}\left(d y_{3}+x_{4} d x_{2}\right) \\
& =d\left(y_{1}+\lambda_{1} y_{2}+\lambda_{2} y_{3}\right)-y_{2} d \lambda_{1}-y_{3} d \lambda_{2}+\left(x_{4}+\lambda_{1} x_{3}\right) d x_{1}+\left(x_{3}+\lambda_{2} x_{4}\right) d x_{2} \\
& =d Z-P_{1} d X_{1}-P_{2} d X_{2}-P_{3} d X_{3}-P_{4} d X_{4} .
\end{aligned}
$$

Thus, putting

$$
\left\{\begin{array}{l}
Z=y_{1}+\lambda_{1} y_{2}+\lambda_{2} y_{3}, X_{1}=\lambda_{1}, X_{2}=\lambda_{2}, X_{3}=x_{1}, X_{4}=x_{2} \\
P_{1}=y_{2}, P_{2}=y_{3}, P_{3}=-\left(x_{4}+\lambda_{1} x_{3}\right), P_{4}=-\left(x_{3}+\lambda_{2} x_{4}\right)
\end{array}\right.
$$

we obtain a canonical coordinate $\left(X_{1}, X_{2}, X_{3}, X_{4}, Z, P_{1}, P_{2}, P_{3}, P_{4}\right)$ of $\left(W(Y), C_{Y}^{*}\right)$.

Conversely we calculate

$$
\left\{\begin{array}{l}
\lambda_{1}=X_{1}, \lambda_{2}=X_{2}, x_{1}=X_{3}, x_{2}=X_{4}, x_{3}=\left(X_{1} X_{2}-1\right)^{-1}\left(P_{4}-X_{2} P_{3}\right), \\
x_{4}=\left(X_{1} X_{2}-1\right)^{-1}\left(P_{3}-X_{1} P_{4}\right), y_{3}=P_{2}, y_{2}=P_{1} .
\end{array}\right.
$$

Hence we have

$$
\left\{\begin{array}{l}
\bar{\theta}_{2}=d y_{2}+x_{3} d x_{1}=d P_{1}+\left(X_{1} X_{2}-1\right)^{-1}\left(P_{4}-X_{2} P_{3}\right) d X_{3} \\
\bar{\theta}_{3}=d y_{3}+x_{4} d x_{2}=d P_{2}+\left(X_{1} X_{2}-1\right)^{-1}\left(P_{3}-X_{1} P_{4}\right) d X_{4} .
\end{array}\right.
$$

Thus we obtain the following description of the model equation of type $\left(C_{3},\left\{\alpha_{2}\right\}\right)$;

$$
\left\{\begin{array}{l}
P_{11}=P_{12}=P_{14}=P_{22}=P_{23}=0, \\
P_{13}=\left(X_{1} X_{2}-1\right)^{-1}\left(X_{2} P_{3}-P_{4}\right), P_{24}=\left(X_{1} X_{2}-1\right)^{-1}\left(X_{1} P_{4}-P_{3}\right) .
\end{array}\right.
$$

Other than $\left(C_{3},\left\{\alpha_{2}\right\}\right)$, we note here that regular differential systems of type $\mathfrak{m}$ satify the condition (Y.2) in $\S 7.1$, when $\mathfrak{m}=\mathfrak{g}_{-2} \oplus \mathfrak{g}_{-1}$ is the negative part of the simple graded Lie algebra of type $\left(C_{\ell},\left\{\alpha_{2}\right\}\right),\left(C_{\ell},\left\{\alpha_{\ell-1}\right\}\right)(\ell \geqq 3),\left(B_{2 m},\left\{\alpha_{2 m}\right\}\right)(p \geqq 2)$ or $\left(F_{4},\left\{\alpha_{4}\right\}\right)$ (cf. $\S 6[12]$, $\S 5.3[24])$.

\section{REFERENCES}

[1] T.N.Baily, Parabolic Invariant Theory and Geometry in "The Penrose Transform and Analytic Cohomology in Representation Theory" Contemp. Math. 154, Amer. Math. Soc., (1993).

[2] W. M. Boothby, Homogeneous complex contact manifolds, Proc. Symp. Pure Math., Amer. Math. Soc. 3 (1961), 144-154.

[3] N.Bourbaki, Groupes et algèbles de Lie, Chapitre 4,5 et 6, Hermann, Paris (1968).

[4] R. Bryant, S. S. Chern, R. B. Gardner, H. Goldschmidt, and P. Griffiths, Exterior differential systems, Springer-Verlag, New-York (1986).

[5] E.Cartan, Les systèmes de Pfaff à cinq variables et les équations aux dérivées partielles du second ordre, Ann. Ec. Normale 27 (1910), 109-192. 
[6] __-_-_, Sur les systèmes en involution d'équations aux dérivées partielles du second ordre à une fonction inconnue de trois variables indépendantes, Bull. Soc. Math. France 39 (1911), 352-443.

[7] J.Hwang and K. Yamaguchi, Characterization of hermitian Symmetric Spaces by Fundamental Forms, Duke Math. J. 120 No.3 (2003), 621-634.

[8] J. E. Humphreys, Introduction to Lie Algebras and Representation Theory, Springer-Verlag, New York (1972).

[9] B. Kostant, Lie algebra cohomology and generalized Borel-Weil theorem, Ann. Math. 74 (1961), $329-387$.

[10] M. Kuranishi, Lectures on involutive systems of partial differential equations, Pub.Soc.Mat., São Paulo (1967).

[11] T. Sasaki, K. Yamaguchi and M. Yoshida, On the Rigidity of Differential Systems modeled on Hermitian Symmetric Spaces and Disproofs of a Conjecture concerning Modular Interpretations of Configuration Spaces, Advanced Studies in Pure Math. 25 (1997), 318-354.

[12] H. Sato and K. Yamaguchi, Lie tensor product manifolds, to appear in Demonstratio Mathematica 45 (2012).

[13] Y. Se-ashi, On differential invariants of integrable finite type linear differential equations, Hokkaido Math.J. 17 (1988), 151-195.

[14] S. Sternberg Lectures on Differential Geometry, Prentice-Hall, New Jersey (1964).

[15] N. Tanaka On generalized graded Lie algebras and geometric structures I, J. Math. Soc. Japan 19 (1967), $215-254$.

[16] _-_- On differential systems, graded Lie algebras and pseudo-groups, J. Math. Kyoto Univ.10 (1970), $1-82$.

[17] _-_, On non-degenerate real hypersurfaces, graded Lie algebras and Cartan connections, Japan. J. Math.2 (1976), 131-190.

[18] _-_-_, On the equivalence problems associated with simple graded Lie algebras, Hokkaido Math. J. 8 (1979), 23-84.

[19] -----, On geometry and integration of systems of second order ordinary differential equations, Proc. Symposium on Differential Geometry, 1982, 194-205 (in Japanese)

[20] K.Yamaguchi Contact geometry of higher order, Japanese J. of Math. 8 (1982), 109-176.

[21] ----- , On involutive systems of second order of codimension 2, Proc. of Japan Acad. 58, Ser A, No.7 (1982), 302-305.

[22] ----- , Geometrization of Jet bundles, Hokkaido Math. J. 12 (1983), 27-40.

[23] __-_, Typical classes in involutive systems of second order, Japanese J. Math. 11 (1985), 265-291.

[24] --_--, Differential systems associated with simple graded Lie algebras, Adv. Studies in Pure Math. 22 (1993), 413-494.

[25] -----, $G_{2}$-Geometry of Overdetermined Systems of Second Order, Trends in Math. (Analysis and Geometry in Several Complex Variables) (1999), Birkhäuser, Boston 289-314.

[26] -----, Geometry of Linear Differential Systems Towards Contact Geometry of Second Order, IMA Volumes in Mathematics and its Applications 144 (Symmetries and Overdetermined Systems of Partial Differential Equations) (2007), 151-203.

[27] _---, Contact Geometry of Second Order I, Differential Equations: Geometry, Symmetries and Integrability: The Abel Symposium 2008, Abel Symposia 5 (2009), 335-386

[28] K. Yamaguchi and T. Yatsui, Geometry of Higher Order Differential Equations of Finite Type associated with Symmetric Spaces, Advanced Studies in Pure Mathematics 37 (2002), 397-458.

[29] K.Yamaguchi and T.Yatsui, Parabolic Geometries associated with Differential Equations of Finite Type, Progress in Mathematics 252 (From Geometry to Quantum Mechanics: In Honor of Hideki Omori) (2007), 161-209

K.Yamaguchi, Department of Mathematics,, Faculty of Science,, Hokkaido University,, SapPORO 060-0810,, JAPAN, E-MAIL yamaguch@math.sci.hokudai.ac.jp 